\title{
Ynamides as racemization-free coupling reagents for amide and peptide synthesis
}

Long $\mathrm{Hu},{ }^{\dagger}$ Silin $\mathrm{Xu},{ }^{\dagger}$ Zhenguang Zhao,${ }^{\dagger}$ Yang Yang, Zhiyuan Peng, Ming Yang, Changliu Wang, Junfeng Zhao*

Key Laboratory of Chemical Biology of Jiangxi Province, College of Chemistry and Chemical Engineering, Jiangxi Normal University, Nanchang, 330022, P. R. China

\section{Supporting Information}

\section{Table of}

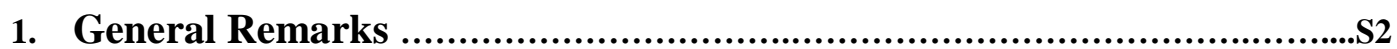

2. General Procedure for the Preparation of Ynamides..............................S3-S5

3. Amide Bond Formation with $\alpha$-Acyloxyenamide as Active Ester...............S6

4. Reaction Condition Optimization for Hydroacyloxylation of Ynamides...S7-S9

5. General Procedure for Hydroacyloxylation of Ynamides with Various Carboxylic Acids S10-S12

6. Control Experiments of Two-step Strategy and One-pot, Two-step Strategy for Amide Bond Formation. S13-S15

7. Ynamide MYTsA Mediated Amide Bond Formation from Carboxylic Acids and Amines.

.S16-S18

8. Comparative Study of Epimerization/Racemization During Dipeptide Synthesis .S19-S31

9. Ynamide MYTsA Mediated Peptide Bond Formation. S32-S38

10. Synthesis of Protected Leu-enkephalin with MYMsA as Coupling Reagent. ..S39-S41

11. NMR Spectra .S42-S119 


\section{General Remarks:}

${ }^{1} \mathrm{H}$ and ${ }^{13} \mathrm{C}$ NMR spectra were recorded on a Bruker (400 and $100 \mathrm{MHz}$ for ${ }^{1} \mathrm{H}$ and ${ }^{13} \mathrm{C}$ respectively) instrument, and are internally referenced to residual solvent signals, $\mathrm{CDCl}_{3}$ referenced at $\delta 7.26$ and 77.00 ppm and DMSO- $\mathrm{d}_{6}$ referenced at $\delta 2.50$ and $39.60 \mathrm{ppm}$. Data for ${ }^{1} \mathrm{H}$ is reported as follows: chemical shift $(\delta \mathrm{ppm})$, integration, multiplicity $(\mathrm{s}=$ singlet, $\mathrm{d}=$ doublet, $\mathrm{t}=$ triplet, $\mathrm{q}=$ quartet, $\mathrm{m}=$ multiplet $)$, broad peaks (br), coupling constant $(\mathrm{Hz})$ and assignment. Data for ${ }^{13} \mathrm{C}$ NMR are reported in terms of chemical shift, multiplicity $(\mathrm{s}=$ singlet, $\mathrm{d}=$ doublet, $\mathrm{t}=$ triplet, $\mathrm{q}=$ quartet, $\mathrm{m}=$ multiplet $)$, coupling constant $(\mathrm{Hz})$ and no special nomenclature is used for equivalent carbons. HRMS (ESI) spectra were obtained by the electrospray ionization time-of-flight (ESI-TOF) mass spectrometry. The diastereomeric ratio (dr) was determined by chiral HPLC with chiral IC column with hexane and $i$-PrOH as eluent. Flash column chromatography purification of compound was carried out by gradient elution using ethyl acetate (EA) in light petroleum ether (PE).Unless otherwise noted, materials obtained from commercial suppliers were used without further purification. 


\section{General Procedure for the Preparation of Ynamides}

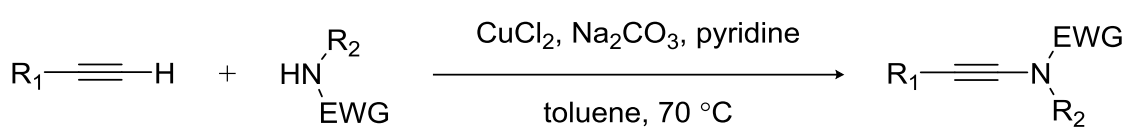

In a $500 \mathrm{~mL}$ three-neck round-bottom flask equipped with a stir-bar, $\mathrm{CuCl}_{2}(2.0 \mathrm{mmol}, 270 \mathrm{mg})$, nitrogen nucleophile $(50.0 \mathrm{mmol})$ and $\mathrm{Na}_{2} \mathrm{CO}_{3}(20.0 \mathrm{mmol}, 2.12 \mathrm{~g})$ were combined. The reaction flask was purged with oxygen gas for 15 minutes. A solution of pyridine $(20.0 \mathrm{mmol})$ in $40.0 \mathrm{ml}$ of toluene was added to the reaction flask via a syringe. Two balloons filled with oxygen gas were connected to the reaction flask via needles. The flask was placed in an oil-bath and heated to $70^{\circ} \mathrm{C}$. A solution of terminal alkyne $(10.0$ mmol) in $20.0 \mathrm{ml}$ of toluene was added slowly to the flask over $4 \mathrm{~h}$ by using a syringe pump. After addition of terminal alkyne/toluene solution, the reaction mixture was allowed to stir at $70{ }^{\circ} \mathrm{C}$ for another 16 hours and then cooled to room temperature. The crude mixture was concentrated under vacuumand then purified by flash chromatography on silica gel with PE/ethyl acetate to afford the ynamide. The unreacted nitrogen nucleophile was recovered.

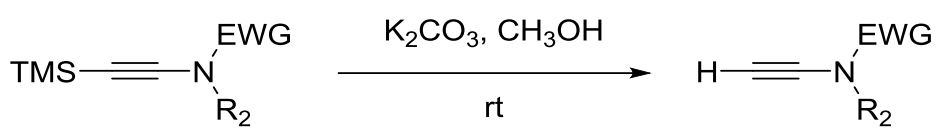

In a $10 \mathrm{ml}$ round-bottom flask equipped with TMS-ynamide (1.0 equiv), $\mathrm{CH}_{3} \mathrm{OH}$ and $\mathrm{K}_{2} \mathrm{CO}_{3}$ (2.0 equiv) were combined. The reaction mixture was stirred at room temperature under air until TMS-ynamide was fully consumed. The reaction mixture was treated with $\mathrm{H}_{2} \mathrm{O}$ at room temperature and aqueous layer was extracted with $\mathrm{Et}_{2} \mathrm{O}$ for three times. The combined organic layers were washed with brine, dried over $\mathrm{Na}_{2} \mathrm{SO}_{4}$, filtered and evaporated in vacuo. Crude product was purified by flash chromatography to afford the terminal ynamide in quantitative yield. 


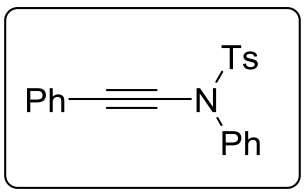

348.1056.

${ }^{1} \mathrm{H}$ NMR (400 MHz, $\left.\mathrm{CDCl}_{3}\right) \delta 7.65(\mathrm{~d}, J=8.3 \mathrm{~Hz}, 2 \mathrm{H}), 7.44-7.38(\mathrm{~m}, 2 \mathrm{H})$, $7.37-7.27(\mathrm{~m}, 10 \mathrm{H}), 2.45(\mathrm{~s}, 3 \mathrm{H}) ;{ }^{13} \mathrm{C}$ NMR (100 MHz, $\left.\mathrm{CDCl}_{3}\right) \delta$ 145.0, 139.0, 133.0, 131.4, 129.5, 129.0, 128.2, 128.2, 127.9, 126.2, 122.6, 83.0, 70.5, 21.6 ppm; HRMS m/z (ESI) calcd for $\mathrm{C}_{21} \mathrm{H}_{18} \mathrm{NO}_{2} \mathrm{~S}(\mathrm{M}+\mathrm{H})^{+}$: 348.1053 , found

N-benzyl-4-methyl-N-(phenylethynyl)benzenesulfonamide

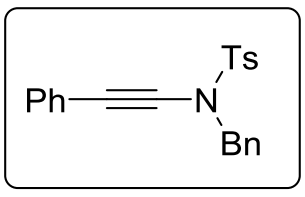

$\mathrm{Bn}$

${ }^{1} \mathrm{H}$ NMR $\left(400 \mathrm{MHz}, \mathrm{CDCl}_{3}\right) \delta 7.76(\mathrm{t}, J=6.5 \mathrm{~Hz}, 2 \mathrm{H}), 7.36-7.23(\mathrm{~m}, 7 \mathrm{H}), 7.20$ -7.19 (m, 5H), 4.54 (s, 2H), 2.39 (s, 3H); ${ }^{13} \mathrm{C} \mathrm{NMR}\left(100 \mathrm{MHz}, \mathrm{CDCl}_{3}\right) \delta 144.6$, 134.8, 134.5, 131.1, 129.7, 128.9, 128.5, 128.3, 128.2, 127.8, 127.6, 122.8, 82.7, 71.4, 55.7, $21.6 \mathrm{ppm}$; HRMS $\mathrm{m} / \mathrm{z}$ (ESI) calcd for $\mathrm{C}_{22} \mathrm{H}_{20} \mathrm{NO}_{2} \mathrm{~S}(\mathrm{M}+\mathrm{H})^{+}$:

362.1209 , found 362.1212 .

\section{N,4-dimethyl-N-(phenylethynyl)benzenesulfonamide}

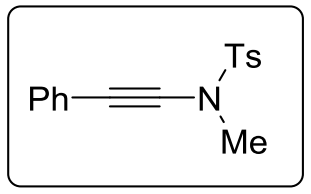

286.0901 .
${ }^{1} \mathrm{H}$ NMR $\left(400 \mathrm{MHz}, \mathrm{CDCl}_{3}\right) \delta 7.85(\mathrm{~d}, J=8.3 \mathrm{~Hz}, 2 \mathrm{H}), 7.42-7.33(\mathrm{~m}, 4 \mathrm{H})$, $7.31-7.26$ (m, 3H), 3.15 (s, 3H), 2.46 (s, 3H); ${ }^{13} \mathrm{C}$ NMR $\left(100 \mathrm{MHz}, \mathrm{CDCl}_{3}\right) \delta$ 144.8, 133.3, 131.4, 129.8, 128.2, 127.8, 127.5, 122.7, 84.0, 69.0, 39.3, 21.6 ppm; HRMS m/z (ESI) calcd for $\mathrm{C}_{16} \mathrm{H}_{16} \mathrm{NO}_{2} \mathrm{~S}(\mathrm{M}+\mathrm{H})^{+}:$286.0896, found

\section{N-methyl-N-(phenylethynyl)methanesulfonamide}

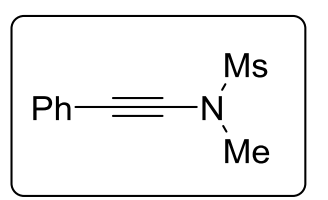

${ }^{1} \mathrm{H}$ NMR (400 MHz, $\left.\mathrm{CDCl}_{3}\right) \delta 7.45-7.38(\mathrm{~m}, 2 \mathrm{H}), 7.33-7.27(\mathrm{~m}, 3 \mathrm{H}), 3.29$ (s, 3H), 3.12 (s, 3H); ${ }^{13} \mathrm{C}$ NMR (100 MHz, $\left.\mathrm{CDCl}_{3}\right) \delta 131.4,128.3,128.0,122.3$, 83.0, 69.4, 39.1, $36.7 \mathrm{ppm}$; HRMS m/z (ESI) calcd for $\mathrm{C}_{10} \mathrm{H}_{12} \mathrm{NO}_{2} \mathrm{~S}(\mathrm{M}+\mathrm{H})^{+}$: 210.0583 , found 210.0588 .

N-(hex-1-yn-1-yl)-N-methylmethanesulfonamide

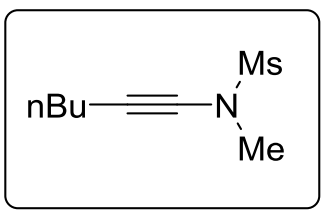

${ }^{1} \mathrm{H}$ NMR (400 MHz, $\left.\mathrm{CDCl}_{3}\right) \delta 3.15(\mathrm{~s}, 3 \mathrm{H}), 3.04(\mathrm{~s}, 3 \mathrm{H}), 2.28(\mathrm{t}, J=7.0 \mathrm{~Hz}$, $2 \mathrm{H}), 1.55-1.44(\mathrm{~m}, 2 \mathrm{H}), 1.40(\mathrm{dq}, J=14.1,7.2 \mathrm{~Hz}, 2 \mathrm{H}), 0.92(\mathrm{t}, J=7.2 \mathrm{~Hz}$, $3 \mathrm{H}),{ }^{13} \mathrm{C}$ NMR $\left(100 \mathrm{MHz}, \mathrm{CDCl}_{3}\right)$ 874.0, 68.9, 39.0, 35.6, 30.8, 21.7, 17.8, $13.4 \mathrm{ppm}$; HRMS m/z (ESI) calcd for $\mathrm{C}_{8} \mathrm{H}_{16} \mathrm{NO}_{2} \mathrm{~S}(\mathrm{M}+\mathrm{H})^{+}: 190.0896$, found 190.0901 .

\section{N,4-dimethyl-N-((trimethylsilyl)ethynyl)benzenesulfonamide}

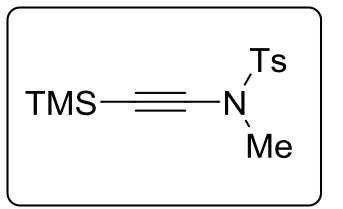

${ }^{1} \mathrm{H}$ NMR $\left(400 \mathrm{MHz}, \mathrm{CDCl}_{3}\right) \delta 7.78(\mathrm{~d}, J=8.3 \mathrm{~Hz}, 2 \mathrm{H}), 7.36(\mathrm{~d}, J=8.0 \mathrm{~Hz}$, 2H), 3.04 (s, 3H), 2.46 (s, 3H), 0.15 (s, 9H); ${ }^{13} \mathrm{C}$ NMR (100 MHz, $\left.\mathrm{CDCl}_{3}\right) \delta$ 144.7, 133.1, 129.6, 127.9, 96.6, 71.3, 39.0, 21.7, 0.1 ppm; HRMS m/z (ESI) calcd for $\mathrm{C}_{13} \mathrm{H}_{20} \mathrm{NO}_{2} \mathrm{SSi}(\mathrm{M}+\mathrm{H})^{+}: 282.0979$, found 282.0984

N-benzyl-N-((trimethylsilyl)ethynyl)methanesulfonamide

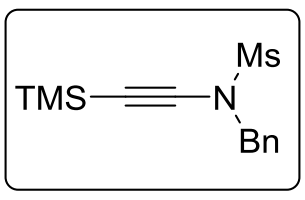

${ }^{1} \mathrm{H}$ NMR (400 MHz, $\left.\mathrm{CDCl}_{3}\right) \delta 7.45-7.40(\mathrm{~m}, 2 \mathrm{H}), 7.40-7.35(\mathrm{~m}, 3 \mathrm{H}), 4.60$ (s, 2H), 2.86 (s, 3H), 0.15 (s, 9H); $\left.{ }^{13} \mathrm{C} \mathrm{NMR} \mathrm{(100} \mathrm{MHz,} \mathrm{CDCl}_{3}\right) \delta$ 134.4, 129.0, 128.7, 128.7, 94.6, 74.3, 55.6, 38.7, -0.1 ppm; HRMS m/z (ESI) calcd for $\mathrm{C}_{13} \mathrm{H}_{20} \mathrm{NO}_{2} \mathrm{SSi}(\mathrm{M}+\mathrm{H})^{+}:$282.0979, found 282.0984.

2-((trimethylsilyl)ethynyl)isoindoline-1,3-dione 


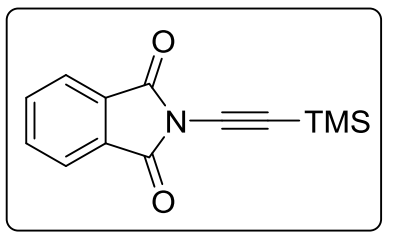

${ }^{1} \mathrm{H}$ NMR (400 MHz, $\left.\mathrm{CDCl}_{3}\right) \delta 7.87(\mathrm{dd}, J=5.5,3.1 \mathrm{~Hz}, 2 \mathrm{H}), 7.78(\mathrm{dd}, J$ $=5.6,3.0 \mathrm{~Hz}, 2 \mathrm{H}), 0.23(\mathrm{~s}, 9 \mathrm{H}) ;{ }^{13} \mathrm{C} \mathrm{NMR}\left(100 \mathrm{MHz}, \mathrm{CDCl}_{3}\right) \delta 164.8$, $135.1,130.9,124.2,84.5,82.9,-0.2 \mathrm{ppm} ; \mathrm{HRMS} \mathrm{m} / \mathrm{z}$ (ESI) calcd for $\mathrm{C}_{13} \mathrm{H}_{14} \mathrm{NO}_{2} \mathrm{Si}(\mathrm{M}+\mathrm{H})^{+}:$244.0788, found 244.0790.

\section{3-((trimethylsilyl)ethynyl)oxazolidin-2-one}

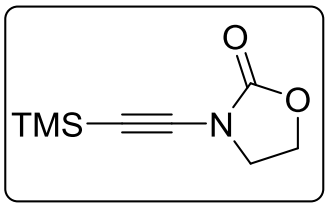

${ }^{1} \mathrm{H} \mathrm{NMR}\left(400 \mathrm{MHz}, \mathrm{CDCl}_{3}\right) \delta 4.40(\mathrm{dd}, J=8.3,7.7 \mathrm{~Hz}, 2 \mathrm{H}), 3.91(\mathrm{dd}, J=8.6$, $7.4 \mathrm{~Hz}, 2 \mathrm{H}), 0.16(\mathrm{~s}, 9 \mathrm{H}) ;{ }^{13} \mathrm{C} \mathrm{NMR}\left(100 \mathrm{MHz}, \mathrm{CDCl}_{3}\right) \delta$ 155.8, 91.3, 73.6, 63.0, 46.8, -0.1 ppm; HRMS m/z (ESI) calcd for $\mathrm{C}_{8} \mathrm{H}_{14} \mathrm{NO}_{2} \mathrm{Si}(\mathrm{M}+\mathrm{H})^{+}$: 184.0788 , found 184.0792 .

N-ethynyl-N,4-dimethylbenzenesulfonamide

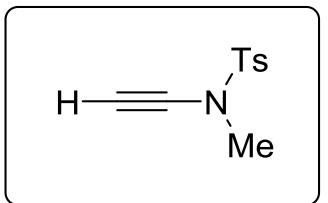

${ }^{1} \mathrm{H}$ NMR (400 MHz, $\left.\mathrm{CDCl}_{3}\right) \delta 7.80(\mathrm{~d}, J=8.3 \mathrm{~Hz}, 2 \mathrm{H}), 7.36(\mathrm{t}, J=7.4 \mathrm{~Hz}$, 2H), 3.06 (s, 3H), $2.68(\mathrm{~s}, 1 \mathrm{H}), 2.46(\mathrm{~s}, 3 \mathrm{H}) .{ }^{13} \mathrm{C} \mathrm{NMR}\left(100 \mathrm{MHz}, \mathrm{CDCl}_{3}\right) \delta$ 144.9, 133.2, 129.8, 127.8, 77.6, 57.4, 38.8, 21.6 ppm; HRMS m/z (ESI) calcd for $\mathrm{C}_{10} \mathrm{H}_{11} \mathrm{NO}_{2} \mathrm{~S}(\mathrm{M}+\mathrm{H})^{+}:$209.0583, found 209.0580.

N-benzyl-N-ethynylmethanesulfonamide

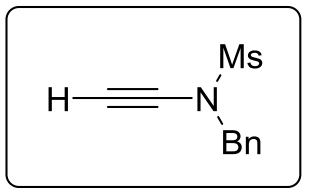

${ }^{1} \mathrm{H}$ NMR (400 MHz, $\left.\mathrm{CDCl}_{3}\right) \delta 7.49-7.33(\mathrm{~m}, 5 \mathrm{H}), 4.62(\mathrm{~s}, 2 \mathrm{H}), 2.89$ (s, 3H), $2.84(\mathrm{~s}, 1 \mathrm{H}) ;{ }^{13} \mathrm{C} \mathrm{NMR}\left(100 \mathrm{MHz}, \mathrm{CDCl}_{3}\right) \delta 134.3,128.8,128.7,75.8,60.1$, 55.3, $38.8 \mathrm{ppm}$; HRMS m/z (ESI) calcd for $\mathrm{C}_{10} \mathrm{H}_{11} \mathrm{NO}_{2} \mathrm{~S}(\mathrm{M}+\mathrm{H})^{+}:$209.0583, found 209.0580 .

N-ethynyl-N-methylmethanesulfonamide

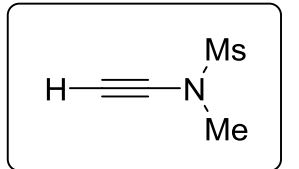

${ }^{1} \mathrm{H}$ NMR (400 MHz, $\mathrm{CDCl}_{3}$ ) $\delta 3.20$ (s, 3H), 3.08 (s, 3H), $2.78(\mathrm{~s}, 1 \mathrm{H}) ;{ }^{13} \mathrm{C}$ NMR $\left(100 \mathrm{MHz}, \mathrm{CDCl}_{3}\right) \delta 76.8,58.1,38.7,36.6 \mathrm{ppm} ; \mathrm{HRMS} \mathrm{m} / \mathrm{z}$ (ESI) calcd for $\mathrm{C}_{4} \mathrm{H}_{8} \mathrm{NO}_{2} \mathrm{~S}(\mathrm{M}+\mathrm{H})^{+}:$134.0270, found 134.0274.

\section{2-ethynylisoindoline-1,3-dione}

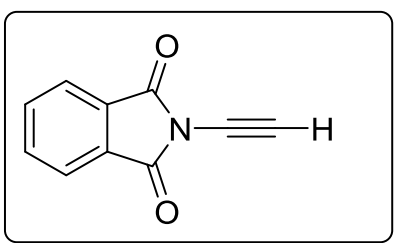

${ }^{1} \mathrm{H}$ NMR $\left(400 \mathrm{MHz}, \mathrm{CDCl}_{3}\right) \delta 7.93(\mathrm{dd}, J=5.4,3.1 \mathrm{~Hz}, 2 \mathrm{H}), 7.85-7.80$ $(\mathrm{m}, 2 \mathrm{H}), 3.33$ (s, $1 \mathrm{H}) ;{ }^{13} \mathrm{C}$ NMR $\left(100 \mathrm{MHz}, \mathrm{CDCl}_{3}\right) \delta 164.9,135.3,130.9$, 124.4, 67.9, $66.2 \mathrm{ppm}$; HRMS m/z (ESI) calcd for $\mathrm{C}_{10} \mathrm{H}_{6} \mathrm{NO}_{2}(\mathrm{M}+\mathrm{H})^{+}$: 172.0393, found 172.0397 . 


\section{Amide Bond Formation with $\alpha$-Acyloxyenamide as Active Ester}

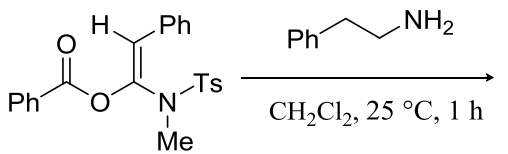<smiles>O=C(NCC[PH2+][18OH])c1ccccc1</smiles>

$2,99 \%$

3, $99 \%$

A $5 \mathrm{~mL}$ round-bottomed flask was charged with $\alpha$-acyloxyenamide $1(0.2 \mathrm{mmol}), \mathrm{CH}_{2} \mathrm{Cl}_{2}(1 \mathrm{~mL})$ and amine $(0.2 \mathrm{mmol})$. The reaction mixture was stirred at room temperature under air until starting material was fully consumed. The reaction mixture was concentrated and purified by silica gel chromatography to afford the amides $\mathbf{2}$ and $\mathbf{3}$ in quantitative yields.

\section{(E)-1-((N,4-dimethylphenyl)sulfonamido)-2-phenylvinyl benzoate (1)}

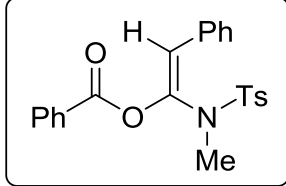

White solid. ${ }^{1} \mathrm{H}$ NMR $\left(400 \mathrm{MHz}, \mathrm{CDCl}_{3}\right) \delta 7.82(\mathrm{~d}, J=8.3 \mathrm{~Hz}, 2 \mathrm{H}), 7.70(\mathrm{~d}, J=$ $7.8 \mathrm{~Hz}, 2 \mathrm{H}), 7.65-7.55(\mathrm{~m}, 3 \mathrm{H}), 7.43-7.37$ (m, 4H), $7.34-7.28(\mathrm{~m}, 1 \mathrm{H}), 7.02$ $(\mathrm{d}, J=8.0 \mathrm{~Hz}, 2 \mathrm{H}), 6.35(\mathrm{~s}, 1 \mathrm{H}), 3.14(\mathrm{~d}, J=0.8 \mathrm{~Hz}, 3 \mathrm{H}), 2.24(\mathrm{~s}, 3 \mathrm{H}) ;{ }^{13} \mathrm{C} \mathrm{NMR}$ $\left(100 \mathrm{MHz} \mathrm{CDCl}_{3}\right) \delta 164.6,143.6,139.9,136.0,133.7,131.9,130.0,129.4,128.6$, 128.6, 128.4, 128.3, 127.5, 119.3, 36.6, $21.3 \mathrm{ppm}$; HRMS m/z (ESI) calcd for $\mathrm{C}_{23} \mathrm{H}_{21} \mathrm{NNaO}_{4} \mathrm{~S}(\mathrm{M}+\mathrm{Na})^{+}$: 430.1083 , found 430.1082 .

N-phenethylbenzamide (2)

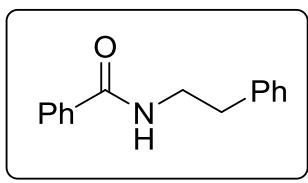

White solid, 99\% yield (44.6 mg). ${ }^{1} \mathrm{H}$ NMR (400 MHz, $\left.\mathrm{CDCl}_{3}\right) \delta 7.69(\mathrm{~d}, J=$ $7.5 \mathrm{~Hz}, 2 \mathrm{H}), 7.46$ (t, $J=7.3 \mathrm{~Hz}, 1 \mathrm{H}), 7.38(\mathrm{t}, J=7.3 \mathrm{~Hz}, 2 \mathrm{H}), 7.31(\mathrm{t}, J=7.2$ $\mathrm{Hz}, 2 \mathrm{H}), 7.23(\mathrm{t}, J=8.0 \mathrm{~Hz}, 3 \mathrm{H}), 6.36(\mathrm{~s}, 1 \mathrm{H}), 3.70(\mathrm{q}, J=6.3 \mathrm{~Hz}, 2 \mathrm{H}), 2.92(\mathrm{t}$, $J=6.8 \mathrm{~Hz}, 2 \mathrm{H}) ;{ }^{13} \mathrm{C}$ NMR $\left(100 \mathrm{MHz}, \mathrm{CDCl}_{3}\right) \delta 167.5,138.9,134.6,131.3$, 128.7, 128.6, 128.5, 126.8, 126.5, 41.1, $35.6 \mathrm{ppm}$; HRMS m/z (ESI) calcd for $\mathrm{C}_{15} \mathrm{H}_{15} \mathrm{NNaO}(\mathrm{M}+\mathrm{Na})^{+}$: 248.1046, found 248.1039 .

\section{N-methyl-2-phenyl-N-tosylacetamide (3)}<smiles>CN(C)C(=O)Cc1ccccc1</smiles>

White solid, 99\% yield (60.0 mg). ${ }^{1} \mathrm{H}$ NMR (400 MHz, $\left.\mathrm{CDCl}_{3}\right) \delta 7.71(\mathrm{~d}, J=8.3$ $\mathrm{Hz}, 2 \mathrm{H}), 7.33-7.28$ (m, 5H), 7.14 (d, J = 6.5 Hz, 2H), 4.06 (s, 2H), 3.29 (s, 3H), $2.45(\mathrm{~s}, 3 \mathrm{H}) ;{ }^{13} \mathrm{C} \mathrm{NMR}\left(100 \mathrm{MHz}, \mathrm{CDCl}_{3}\right) \delta 171.2,144.9,136.0,133.4,129.8$, $129.3,128.5,127.5,127.1,43.0,33.2,21.5 \mathrm{ppm} ; \mathrm{HRMS} \mathrm{m} / \mathrm{z}$ (ESI) calcd for 


\section{Reaction Condition Optimization for Hydroacyloxylation of Ynamides}

Table 1. Hydroacyloxylation of Ynamides with Benzoic Acids

\begin{tabular}{|c|c|c|c|c|c|c|}
\hline $\begin{array}{r}\mathrm{R}^{1} \overline{\overline{ }} \\
4\end{array}$ & $N_{\mathrm{R}^{2}}^{\mathrm{EWG}}$ & + & $\mathrm{OH}$ & $\frac{\text { Solvent }}{25^{\circ} \mathrm{C}}$ & \rangle$_{\mathrm{H}}^{\mathrm{R}^{1}}$ & $=\mathrm{S}_{\mathrm{O}}^{\mathrm{N}-\mathrm{EWG}}$ \\
\hline entry & EWG & $\mathrm{R}^{1}$ & $\mathrm{R}^{2}$ & solvent & time (h) & yield $(\%)$ \\
\hline 1 & Ts & $\mathrm{Ph}$ & $\mathrm{Me}$ & THF & 48 & trace \\
\hline 2 & Ts & $\mathrm{Ph}$ & $\mathrm{Me}$ & DMF & 48 & NR \\
\hline 3 & Ts & $\mathrm{Ph}$ & $\mathrm{Me}$ & DMA & 48 & NR \\
\hline 4 & Ts & $\mathrm{Ph}$ & $\mathrm{Me}$ & DMSO & 48 & $43 \%$ \\
\hline 5 & Ts & $\mathrm{Ph}$ & $\mathrm{Me}$ & $\mathrm{MeCN}$ & 48 & $52 \%$ \\
\hline 6 & Ts & $\mathrm{Ph}$ & $\mathrm{Me}$ & acetone & 48 & $81 \%$ \\
\hline 7 & Ts & $\mathrm{Ph}$ & $\mathrm{Me}$ & $\mathrm{CH}_{3} \mathrm{OH}$ & 48 & $78 \%$ \\
\hline 8 & Ts & $\mathrm{Ph}$ & $\mathrm{Me}$ & $\mathrm{CHCl}_{3}$ & 48 & $91 \%$ \\
\hline 9 & Ts & $\mathrm{Ph}$ & $\mathrm{Me}$ & DCE & 48 & $56 \%$ \\
\hline 10 & Ts & $\mathrm{Ph}$ & $\mathrm{Me}$ & toluene & 20 & $72 \%$ \\
\hline 11 & Ts & $\mathrm{Ph}$ & $\mathrm{Me}$ & DCM & 15 & $93 \%$ \\
\hline 12 & Ts & $\mathrm{Ph}$ & $\mathrm{Ph}$ & $\mathrm{DCM}$ & 24 & $38 \%$ \\
\hline 13 & Ts & $\mathrm{Ph}$ & $\mathrm{Bn}$ & DCM & 20 & $90 \%$ \\
\hline 14 & Ts & TMS & $\mathrm{Me}$ & DCM & 12 & $92 \%$ \\
\hline 15 & Ts & $\mathrm{H}$ & $\mathrm{Me}$ & DCM & 1.5 & $99 \%$ \\
\hline 16 & Ms & $\mathrm{Ph}$ & $\mathrm{Me}$ & DCM & 20 & $90 \%$ \\
\hline 17 & Ms & TMS & $\mathrm{Bn}$ & DCM & 17 & $88 \%$ \\
\hline 18 & Ms & $\mathrm{H}$ & $\mathrm{Bn}$ & DCM & 5 & $91 \%$ \\
\hline 19 & Ms & TMS & $\mathrm{Me}$ & $\mathrm{DCM}$ & 17 & $89 \%$ \\
\hline 20 & Ms & $\mathrm{n}-\mathrm{Bu}$ & $\mathrm{Me}$ & DCM & 12 & $92 \%$ \\
\hline 21 & Ms & $\mathrm{H}$ & $\mathrm{Me}$ & DCM & 1.5 & $99 \%$ \\
\hline 22 & $\mathrm{H}=$ & & & DCM & 12 & $53 \%$ \\
\hline 23 & 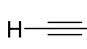 & & & DCM & 12 & 0 \\
\hline
\end{tabular}

To a $5 \mathrm{~mL}$ round-bottomed flask was added ynamide $(0.2 \mathrm{mmol}), \mathrm{CH}_{2} \mathrm{Cl}_{2}(1 \mathrm{~mL})$ and benzoic acid $(0.2$ $\mathrm{mmol})$. The reaction mixture was stirred at room temperature under air until starting material was fully consumed. The reaction mixture was concentrated and purified by silica gel chromatography to afford the $\alpha$-acyloxyenamides. 
(E)-1-((4-methyl-N-phenylphenyl)sulfonamido)-2-phenylvinyl benzoate (5a)

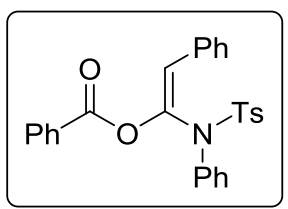

White solid, 38\% yield (35.7 mg). ${ }^{1} \mathrm{H}$ NMR (400 MHz, $\left.\mathrm{CDCl}_{3}\right) \delta 7.88(\mathrm{~d}, J=7.5$ $\mathrm{Hz}, 2 \mathrm{H}), 7.67-7.61(\mathrm{~m}, 3 \mathrm{H}), 7.52(\mathrm{~d}, J=8.2 \mathrm{~Hz}, 2 \mathrm{H}), 7.44(\mathrm{t}, J=7.7 \mathrm{~Hz}, 2 \mathrm{H})$, $7.37-7.28(\mathrm{~m}, 5 \mathrm{H}), 7.24-7.20(\mathrm{~m}, 3 \mathrm{H}), 7.04(\mathrm{~d}, J=8.1 \mathrm{~Hz}, 2 \mathrm{H}), 6.65(\mathrm{~s}, 1 \mathrm{H})$, $2.31(\mathrm{~s}, 3 \mathrm{H}) ;{ }^{13} \mathrm{C}$ NMR $\left(100 \mathrm{MHz}, \mathrm{CDCl}_{3}\right) \delta 163.5,143.9,139.2,138.7,136.5$, 133.7, 132.2, 130.1, 129.2, 129.0, 129.0, 128.5, 128.4, 128.2, 128.2, 127.8, 127.0, 122.1, 21.5 ppm; HRMS m/z (ESI) calcd for $\mathrm{C}_{28} \mathrm{H}_{23} \mathrm{NNaO}_{4} \mathrm{~S}(\mathrm{M}+\mathrm{Na})^{+}: 492.1240$, found 492.1237.

(E)-1-((N-benzyl-4-methylphenyl)sulfonamido)-2-phenylvinyl benzoate (5b)

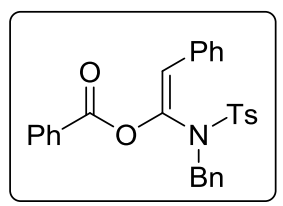

White solid, 90\% yield (87 mg). ${ }^{1} \mathrm{H}$ NMR (400 MHz, $\left.\mathrm{CDCl}_{3}\right) \delta 7.80-7.70(\mathrm{~m}$, $4 \mathrm{H}), 7.59(\mathrm{t}, J=7.5 \mathrm{~Hz}, 1 \mathrm{H}), 7.39(\mathrm{t}, J=7.8 \mathrm{~Hz}, 2 \mathrm{H}), 7.35-7.33(\mathrm{~m}, 2 \mathrm{H}), 7.25-$ $7.07(\mathrm{~m}, 10 \mathrm{H}), 6.51(\mathrm{~s}, 1 \mathrm{H}), 4.52(\mathrm{~s}, 2 \mathrm{H}), 2.34(\mathrm{~s}, 3 \mathrm{H}) ;{ }^{13} \mathrm{C} \mathrm{NMR}\left(100 \mathrm{MHz}, \mathrm{CDCl}_{3}\right)$ $\delta 163.9,143.8,137.0,136.8,134.5,133.6$ 132.0, 130.0, 129.7, 129.5, 129.1, 128.8,

128.3, 128.2, 128.1, 128.0, 127.8, 123.4, 52.8, 21.4 ppm; HRMS m/z (ESI) calcd for $\mathrm{C}_{29} \mathrm{H}_{25} \mathrm{NNaO}_{4} \mathrm{~S}$ $(\mathrm{M}+\mathrm{Na})^{+}:$506.1397, found 506.1384.

(E)-1-((N,4-dimethylphenyl)sulfonamido)-2-phenylvinyl benzoate (5c)

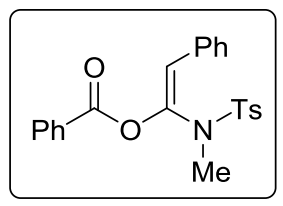

White solid, 93\% yield (80.6 mg). ${ }^{1} \mathrm{H}$ NMR $\left(400 \mathrm{MHz}, \mathrm{CDCl}_{3}\right) \delta 7.82(\mathrm{~d}, J=8.3$ $\mathrm{Hz}, 2 \mathrm{H}), 7.70(\mathrm{~d}, J=7.8 \mathrm{~Hz}, 2 \mathrm{H}), 7.65-7.55(\mathrm{~m}, 3 \mathrm{H}), 7.43-7.37(\mathrm{~m}, 4 \mathrm{H}), 7.34$ $-7.28(\mathrm{~m}, 1 \mathrm{H}), 7.02(\mathrm{~d}, J=8.0 \mathrm{~Hz}, 2 \mathrm{H}), 6.35(\mathrm{~s}, 1 \mathrm{H}), 3.14(\mathrm{~d}, J=0.8 \mathrm{~Hz}, 3 \mathrm{H})$, $2.24(\mathrm{~s}, 3 \mathrm{H}) ;{ }^{13} \mathrm{C} \mathrm{NMR}\left(100 \mathrm{MHz}, \mathrm{CDCl}_{3}\right) \delta 164.6,143.6,139.9,136.0,133.7$, 131.9, 130.0, 129.4, 128.6, 128.5, 128.4, 128.3, 127.5, 119.3, 36.6, 21.3 ppm; HRMS m/z (ESI) calcd for $\mathrm{C}_{23} \mathrm{H}_{21} \mathrm{NNaO}_{4} \mathrm{~S}(\mathrm{M}+\mathrm{Na})^{+}$: 430.1083, found 430.1080 .

(E)-1-((N,4-dimethylphenyl)sulfonamido)-2-(trimethylsilyl)vinyl benzoate (5d)

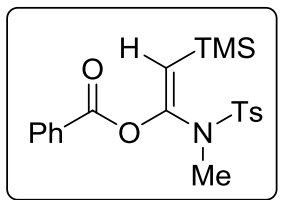

White solid, $92 \%$ yield $(74.3 \mathrm{mg}) .{ }^{1} \mathrm{H}$ NMR $\left(400 \mathrm{MHz}, \mathrm{CDCl}_{3}\right) \delta 7.74(\mathrm{~d}, J=8.2$ $\mathrm{Hz}, 2 \mathrm{H}), 7.56-7.51(\mathrm{~m}, 3 \mathrm{H}), 7.31(\mathrm{t}, J=7.8 \mathrm{~Hz}, 2 \mathrm{H}), 7.16(\mathrm{~d}, J=8.0 \mathrm{~Hz}, 2 \mathrm{H})$, $5.50(\mathrm{~s}, 1 \mathrm{H}), 3.04(\mathrm{~s}, 3 \mathrm{H}), 2.31(\mathrm{~s}, 3 \mathrm{H}), 0.30(\mathrm{~s}, 9 \mathrm{H}) ;{ }^{13} \mathrm{C} \mathrm{NMR}\left(100 \mathrm{MHz}, \mathrm{CDCl}_{3}\right)$ $\delta 163.9,146.2,143.6,135.5,133.4,129.8,129.4,128.8,128.2,128.0,118.5,36.9$, 21.3, -0.8 ppm; HRMS m/z (ESI) calcd for $\mathrm{C}_{20} \mathrm{H}_{25} \mathrm{NNaO}_{4} \mathrm{SSi}(\mathrm{M}+\mathrm{Na})^{+}$: 426.1166, found 426.1157.

\section{1-((N,4-dimethylphenyl)sulfonamido)vinyl benzoate $(5 \mathrm{e})$}

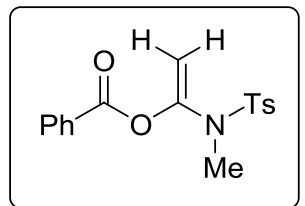

White solid, 99\% yield (66.3 mg). ${ }^{1} \mathrm{H}$ NMR $\left(400 \mathrm{MHz}, \mathrm{CDCl}_{3}\right) \delta 7.86(\mathrm{dd}, J=$ 8.2, $1.0 \mathrm{~Hz}, 2 \mathrm{H}), 7.74(\mathrm{~d}, J=8.3 \mathrm{~Hz}, 2 \mathrm{H}), 7.61-7.55(\mathrm{~m}, 1 \mathrm{H}), 7.41(\mathrm{t}, J=7.8$ $\mathrm{Hz}, 2 \mathrm{H}), 7.28-7.23(\mathrm{~m}, 2 \mathrm{H}), 5.03(\mathrm{~d}, J=2.5 \mathrm{~Hz}, 1 \mathrm{H}), 4.88(\mathrm{~d}, J=2.5 \mathrm{~Hz}, 1 \mathrm{H})$, $3.10(\mathrm{~s}, 3 \mathrm{H}), 2.38(\mathrm{~s}, 3 \mathrm{H}) ;{ }^{13} \mathrm{C} \mathrm{NMR}\left(100 \mathrm{MHz}, \mathrm{CDCl}_{3}\right) \delta 164.0,146.8,144.0$, $134.3,133.7,130.1,129.5,128.6,128.4,127.9,101.7,37.1,21.5$ ppm; HRMS m/z (ESI) calcd for $\mathrm{C}_{17} \mathrm{H}_{17} \mathrm{NNaO}_{4} \mathrm{~S}(\mathrm{M}+\mathrm{Na})^{+}:$354.0770, found 354.0771.

\section{1-(N-methylmethylsulfonamido)vinyl benzoate (5f)}

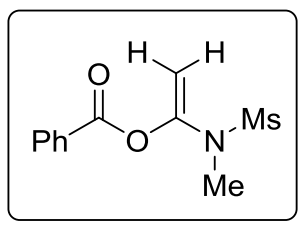

White solid, 99\% yield (51.1 mg). ${ }^{1} \mathrm{H}$ NMR (400 MHz, $\left.\mathrm{CDCl}_{3}\right) \delta 8.09$ (dd, $J=$ $8.3,1.2 \mathrm{~Hz}, 2 \mathrm{H}), 7.68-7.60(\mathrm{~m}, 1 \mathrm{H}), 7.50(\mathrm{t}, J=7.8 \mathrm{~Hz}, 2 \mathrm{H}), 5.15(\mathrm{~d}, J=2.6$ $\mathrm{Hz}, 1 \mathrm{H}), 4.99$ (d, J=2.6 Hz, 1H), 3.18 (s, 3H), 3.03 (s, 3H); ${ }^{13} \mathrm{C}$ NMR (100 MHz, $\left.\mathrm{CDCl}_{3}\right) \delta 164.2,146.1,134.1,130.1,128.8,128.3,100.3,37.7,36.0$ ppm; HRMS $\mathrm{m} / \mathrm{z}(\mathrm{ESI})$ calcd for $\mathrm{C}_{11} \mathrm{H}_{13} \mathrm{NNaO}_{4} \mathrm{~S}(\mathrm{M}+\mathrm{Na})^{+}: 278.0457$, found 278.0453 .

1-(2-oxooxazolidin-3-yl)vinyl benzoate (5g) 


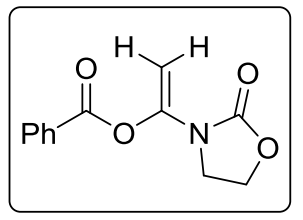

234.0763 .

White solid, 53\% yield $(23.8 \mathrm{mg}) .{ }^{1} \mathrm{H}$ NMR $\left(400 \mathrm{MHz}, \mathrm{CDCl}_{3}\right) \delta 8.16(\mathrm{dd}, J=$ 8.4, $1.3 \mathrm{~Hz}, 2 \mathrm{H}), 7.76-7.70(\mathrm{~m}, 1 \mathrm{H}), 7.60$ (t, $J=7.9 \mathrm{~Hz}, 2 \mathrm{H}), 5.05$ (d, $J=2.5$ $\mathrm{Hz}, 1 \mathrm{H}), 4.81(\mathrm{~d}, J=2.5 \mathrm{~Hz}, 1 \mathrm{H}), 4.40-4.36(\mathrm{~m}, 2 \mathrm{H}), 3.83-3.79(\mathrm{~m}, 2 \mathrm{H}) ;{ }^{13} \mathrm{C} \mathrm{NMR}$ $\left(\mathrm{CDCl}_{3}, 100 \mathrm{MHz}\right) \delta 165.2,155.4,137.6,134.1,132.1,128.8,128.6,116.3,63.1$, $44.7 \mathrm{ppm}$; HRMS (ESI) $\mathrm{m} / \mathrm{z}$ calculated for $\mathrm{C}_{12} \mathrm{H}_{12} \mathrm{NO}_{4}(\mathrm{M}+\mathrm{H})^{+}: 234.0766$ found:

(E)-1-(N-methylmethylsulfonamido)-2-(trimethylsilyl)vinyl benzoate<smiles>CC(=C(OC(=O)c1ccccc1)N(C)C)N(C)C</smiles>

White solid, $89 \%$ yield $(58.8 \mathrm{mg}) .{ }^{1} \mathrm{H}$ NMR $\left(400 \mathrm{MHz}, \mathrm{CDCl}_{3}\right) \delta 8.08-8.02(\mathrm{~m}$, 2H), $7.67-7.60(\mathrm{~m}, 1 \mathrm{H}), 7.49(\mathrm{t}, J=7.7 \mathrm{~Hz}, 2 \mathrm{H}), 5.46(\mathrm{~s}, 1 \mathrm{H}), 3.13(\mathrm{~s}, 3 \mathrm{H}), 2.96$ (s, 3H), 0.24 (s, 9H); $\left.{ }^{13} \mathrm{C} \mathrm{NMR} \mathrm{(100} \mathrm{MHz,} \mathrm{CDCl}_{3}\right) \delta 164.4,146.0,134.0,129.9$, 128.8, 128.6, 119.0, 37.8, 36.9, $-0.9 \mathrm{ppm}$; HRMS m/z (ESI) calcd for $\mathrm{C}_{14} \mathrm{H}_{22} \mathrm{NO}_{4} \mathrm{SSi}(\mathrm{M}+\mathrm{H})^{+}: 328.1039$, found 328.1037 .

(E)-1-(N-methylmethylsulfonamido)-2-phenylvinyl benzoate

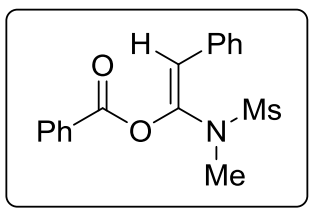

White solid, $90 \%$ yield $(59.6 \mathrm{mg}){ }^{1} \mathrm{H}$ NMR $\left(400 \mathrm{MHz}, \mathrm{CDCl}_{3}\right) \delta 8.19-8.13$ (m, 2H), 7.67 (dd, $J=10.6,4.3 \mathrm{~Hz}, 1 \mathrm{H}), 7.60-7.48(\mathrm{~m}, 4 \mathrm{H}), 7.42-7.38$ (m, 1H), $7.35-7.27$ (m, 2H), 6.37 (s, 1H), 3.16 (s, 3H), 2.90 (s, 3H); ${ }^{13} \mathrm{C} \mathrm{NMR} \mathrm{(100}$ $\left.\mathrm{MHz}, \mathrm{CDCl}_{3}\right) \delta 165.1,139.7,131.8,131.6,130.3,130.2,129.0,128.8,128.6$, 128.5, 119.7, 39.4, $36.3 \mathrm{ppm}$; HRMS m/z (ESI) calcd for $\mathrm{C}_{17} \mathrm{H}_{18} \mathrm{NO}_{4} \mathrm{~S}(\mathrm{M}+\mathrm{H})^{+}: 332.0957$, found 332.0955 .

(E)-1-(N-benzylmethylsulfonamido)-2-(trimethylsilyl)vinyl benzoate<smiles>CN(C)C(OC(=O)c1ccccc1)C1CC2CCC1C2</smiles>

White solid, $88 \%$ yield $(70.9 \mathrm{mg}) .{ }^{1} \mathrm{H}$ NMR $\left(400 \mathrm{MHz}, \mathrm{CDCl}_{3}\right) \delta 8.08-7.98(\mathrm{~m}$, 2H), $7.65(\mathrm{t}, J=7.4 \mathrm{~Hz}, 1 \mathrm{H}), 7.54-7.44(\mathrm{~m}, 4 \mathrm{H}), 7.41-7.29(\mathrm{~m}, 3 \mathrm{H}), 5.54(\mathrm{~s}$, $1 \mathrm{H}), 4.53$ (s, 2H), 2.98 (s, 3H), -0.01 (s, 9H); $\left.{ }^{13} \mathrm{C} \mathrm{NMR} \mathrm{(100} \mathrm{MHz,} \mathrm{CDCl}_{3}\right) \delta$ 164.3, 143.2, 134.7, 133.9, 130.2, 129.8, 129.0, 128.8, 128.5, 128.5, 120.9, 52.8, 40.0, -1.1 ppm; HRMS m/z (ESI) calcd for $\mathrm{C}_{20} \mathrm{H}_{26} \mathrm{NO}_{4} \mathrm{SSi}(\mathrm{M}+\mathrm{H})^{+}:$404.1352, found 404.1350.

\section{1-(N-benzylmethylsulfonamido)vinyl benzoate}

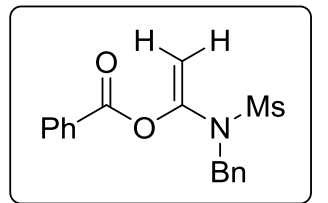

White solid, 92\% yield (60.7 mg). ${ }^{1} \mathrm{H}$ NMR (400 MHz, $\left.\mathrm{CDCl}_{3}\right) \delta 8.05(\mathrm{dd}, J=$ $8.2,1.0 \mathrm{~Hz}, 2 \mathrm{H}), 7.67-7.63(\mathrm{~m}, 1 \mathrm{H}), 7.50(\mathrm{t}, J=7.8 \mathrm{~Hz}, 2 \mathrm{H}), 7.42(\mathrm{~d}, J=6.9$ $\mathrm{Hz}, 2 \mathrm{H}), 7.38-7.28(\mathrm{~m}, 3 \mathrm{H}), 5.07(\mathrm{~d}, J=2.4 \mathrm{~Hz}, 1 \mathrm{H}), 5.00(\mathrm{~d}, J=2.4 \mathrm{~Hz}, 1 \mathrm{H})$, $4.67(\mathrm{~s}, 2 \mathrm{H}), 3.09(\mathrm{~s}, 3 \mathrm{H}) ;{ }^{13} \mathrm{C} \mathrm{NMR}\left(100 \mathrm{MHz}, \mathrm{CDCl}_{3}\right) \delta 164.0,143.7,135.6$, 134.1, 130.1, 128.7, 128.6, 128.6, 128.3, 128.0, 103.4, 51.7, 40.0 ppm; HRMS m/z (ESI) calcd for $\mathrm{C}_{17} \mathrm{H}_{18} \mathrm{NO}_{4} \mathrm{~S}(\mathrm{M}+\mathrm{H})^{+}: \quad 332.0957$, found 332.0956 .

\section{(E)-1-(N-methylmethylsulfonamido)hex-1-en-1-yl benzoate}

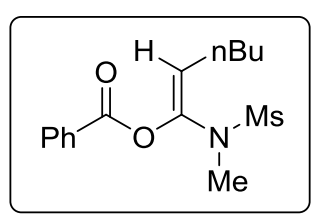

${ }^{1} \mathrm{H}$ NMR (400 MHz, $\left.\mathrm{CDCl}_{3}\right) \delta 8.14-8.04(\mathrm{~m}, 2 \mathrm{H}), 7.63(\mathrm{t}, J=7.5 \mathrm{~Hz}, 1 \mathrm{H})$, $7.48(\mathrm{t}, J=7.8 \mathrm{~Hz}, 2 \mathrm{H}), 5.48(\mathrm{t}, J=7.6 \mathrm{~Hz}, 1 \mathrm{H}), 3.16(\mathrm{~s}, 3 \mathrm{H}), 2.94(\mathrm{~s}, 3 \mathrm{H}), 2.31$ $-2.26(\mathrm{~m}, 7.3 \mathrm{~Hz}, 2 \mathrm{H}), 1.51-1.34(\mathrm{~m}, 4 \mathrm{H}), 0.93(\mathrm{t}, J=7.1 \mathrm{~Hz}, 3 \mathrm{H}) ;{ }^{13} \mathrm{C} \mathrm{NMR}$ $\left(100 \mathrm{MHz}, \mathrm{CDCl}_{3}\right) \delta 165.1,138.9,133.9,130.0,129.1,128.9,128.7,128.6$, $122.9,38.5,36.5,30.8,29.0,28.3,26.6,22.3,13.8 \mathrm{ppm} ; \mathrm{HRMS} \mathrm{m} / \mathrm{z}(\mathrm{ESI})$ calcd for $\mathrm{C}_{15} \mathrm{H}_{22} \mathrm{NO}_{4} \mathrm{~S}(\mathrm{M}+\mathrm{H})^{+}$: 312.1270 , found 312.1272 . 


\section{General Procedure for Hydroacyloxylation of Ynamide with Various Carboxylic}

Acids

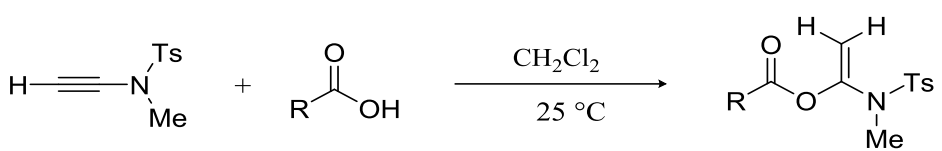

A $5 \mathrm{~mL}$ round-bottomed flask was charged with $\mathrm{N}$-methyl ynetoluenesulfonamide (MYTsA) (0.2 mmol), $\mathrm{CH}_{2} \mathrm{Cl}_{2}(1 \mathrm{~mL})$ and carboxylic acid $(0.2 \mathrm{mmol})$. The reaction mixture was stirred at room temperature under air until starting material was fully consumed. The reaction mixture was concentrated and purified by silica gel chromatography to afford the $\alpha$-acyloxyenamides.

\section{1-((N,4-dimethylphenyl)sulfonamido)vinyl formate (7a)}

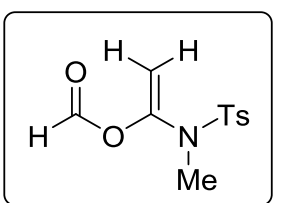

White solid, 99\% yield (50.5 mg). ${ }^{1} \mathrm{H} \mathrm{NMR}\left(400 \mathrm{MHz}, \mathrm{CDCl}_{3}\right) \delta 8.06(\mathrm{~s}, 1 \mathrm{H})$, $7.73(\mathrm{~d}, J=8.3 \mathrm{~Hz}, 2 \mathrm{H}), 7.34(\mathrm{~d}, J=8.0 \mathrm{~Hz}, 2 \mathrm{H}), 4.83(\mathrm{~d}, J=2.9 \mathrm{~Hz}, 1 \mathrm{H}), 4.73$ $(\mathrm{d}, J=2.8 \mathrm{~Hz}, 1 \mathrm{H}), 3.01(\mathrm{~s}, 3 \mathrm{H}), 2.43(\mathrm{~s}, 3 \mathrm{H}) ;{ }^{13} \mathrm{C} \mathrm{NMR}\left(100 \mathrm{MHz}, \mathrm{CDCl}_{3}\right) \delta$ 158.2, 146.9, 144.4, 134.1, 129.6, 127.8, 99.7, 36.8, 21.5 ppm; HRMS m/z (ESI) calcd for $\mathrm{C}_{11} \mathrm{H}_{13} \mathrm{NNaO}_{4} \mathrm{~S}(\mathrm{M}+\mathrm{Na})^{+}:$278.0457, found 278.0452 .

1-((N,4-dimethylphenyl)sulfonamido)vinyl pent-4-enoate (7b)

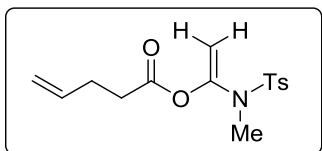

White solid, 92\% yield (56.9 mg). ${ }^{1} \mathrm{H}$ NMR (400 MHz, $\left.\mathrm{CDCl}_{3}\right) \delta 7.70(\mathrm{~d}, J=$ $8.2 \mathrm{~Hz}, 2 \mathrm{H}), 7.31(\mathrm{~d}, J=8.1 \mathrm{~Hz}, 2 \mathrm{H}), 5.81-5.71(\mathrm{~m}, 1 \mathrm{H}), 5.11-4.94(\mathrm{~m}, 2 \mathrm{H})$, $4.79(\mathrm{~d}, J=2.4 \mathrm{~Hz}, 1 \mathrm{H}), 4.62(\mathrm{~d}, J=2.4 \mathrm{~Hz}, 1 \mathrm{H}), 2.99$ (s, 3H), $2.42(\mathrm{~s}, 3 \mathrm{H})$, $2.40(\mathrm{~d}, J=7.7 \mathrm{~Hz}, 2 \mathrm{H}), 2.32(\mathrm{t}, J=6.7 \mathrm{~Hz}, 2 \mathrm{H}) ;{ }^{13} \mathrm{C} \mathrm{NMR}\left(100 \mathrm{MHz}, \mathrm{CDCl}_{3}\right) \delta 170.4,146.9,144.0$, 136.0, 133.9, 129.4, 127.9, 115.7, 100.4, 37.2, 33.1, 28.3, 21.5 ppm; HRMS m/z (ESI) calcd for $\mathrm{C}_{15} \mathrm{H}_{19} \mathrm{NNaO}_{4} \mathrm{~S}(\mathrm{M}+\mathrm{Na})^{+}: 332.0927$, found 332.0937.

1-((N,4-dimethylphenyl)sulfonamido)vinyl adamantane-1-carboxylate (7c)

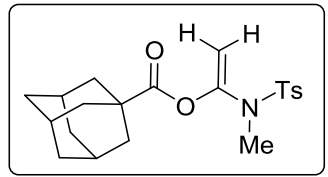

White solid, $98 \%$ yield $(76.3 \mathrm{mg}) .{ }^{1} \mathrm{H}$ NMR $\left(400 \mathrm{MHz}, \mathrm{CDCl}_{3}\right) \delta 7.73(\mathrm{~d}, J=$ $8.3 \mathrm{~Hz}, 2 \mathrm{H}), 7.32(\mathrm{~d}, J=8.1 \mathrm{~Hz}, 2 \mathrm{H}), 4.83(\mathrm{~d}, J=2.2 \mathrm{~Hz}, 1 \mathrm{H}), 4.79(\mathrm{~d}, J=2.2$ $\mathrm{Hz}, 1 \mathrm{H}), 2.95$ (s, 3H), 2.42 (s, 3H), 1.97 (s, 3H), 1.75 (d, $J=2.5 \mathrm{~Hz}, 6 \mathrm{H}), 1.70$ $(\mathrm{d}, J=12.4 \mathrm{~Hz}, 3 \mathrm{H}), 1.63(\mathrm{~d}, J=12.1 \mathrm{~Hz}, 3 \mathrm{H}) ;{ }^{13} \mathrm{C} \mathrm{NMR}\left(100 \mathrm{MHz}, \mathrm{CDCl}_{3}\right)$ $\delta 174.8,146.5,143.9,134.1,129.5,128.1,102.0,41.0,38.3,36.6,36.2,27.7,21.5$ ppm; HRMS m/z (ESI) calcd for $\mathrm{C}_{21} \mathrm{H}_{27} \mathrm{NNaO}_{4} \mathrm{~S}(\mathrm{M}+\mathrm{Na})^{+}:$412.1553, found 412.1550.

\section{1-((N,4-dimethylphenyl)sulfonamido)vinyl 4-chlorobenzoate (7d)}

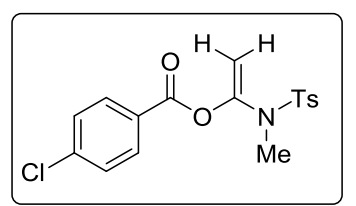

White solid, $98 \%$ yield $(71.5 \mathrm{mg}) .{ }^{1} \mathrm{H}$ NMR $\left(400 \mathrm{MHz}, \mathrm{CDCl}_{3}\right) \delta 7.88-7.78$ (m, 2H), $7.72(\mathrm{~d}, J=8.3 \mathrm{~Hz}, 2 \mathrm{H}), 7.43-7.34(\mathrm{~m}, 2 \mathrm{H}), 7.31-7.20(\mathrm{~m}, 2 \mathrm{H})$, $5.01(\mathrm{~d}, J=2.6 \mathrm{~Hz}, 1 \mathrm{H}), 4.82(\mathrm{~d}, J=2.6 \mathrm{~Hz}, 1 \mathrm{H}), 3.09(\mathrm{~s}, 3 \mathrm{H}), 2.39(\mathrm{~s}, 3 \mathrm{H})$; ${ }^{13} \mathrm{C} \mathrm{NMR}\left(100 \mathrm{MHz}, \mathrm{CDCl}_{3}\right) \delta 163.1,146.9,144.0,140.3,134.1,131.5,129.5$, 128.8, 127.9, 127.1, 101.4, 37.3, $21.5 \mathrm{ppm}$; HRMS m/z (ESI) calcd for $\mathrm{C}_{17} \mathrm{H}_{17} \mathrm{ClNO}_{4} \mathrm{~S}(\mathrm{M}+\mathrm{H})^{+}: 366.0567$, found 366.0567 .

1-((N,4-dimethylphenyl)sulfonamido)vinyl furan-2-carboxylate (7e) 


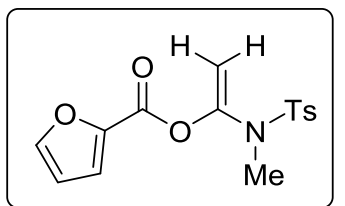

White solid, 98\% yield (63.0 mg). ${ }^{1} \mathrm{H}$ NMR (400 MHz, $\left.\mathrm{CDCl}_{3}\right) \delta 7.66(\mathrm{~d}, J$ $=8.0 \mathrm{~Hz}, 2 \mathrm{H}), 7.54(\mathrm{~s}, 1 \mathrm{H}), 7.20(\mathrm{~d}, J=8.2 \mathrm{~Hz}, 2 \mathrm{H}), 7.02(\mathrm{~d}, J=3.2 \mathrm{~Hz}, 1 \mathrm{H})$, $6.44(\mathrm{~s}, 1 \mathrm{H}), 4.94(\mathrm{~d}, J=2.1 \mathrm{~Hz}, 1 \mathrm{H}), 4.75(\mathrm{~d}, J=2.1 \mathrm{~Hz}, 1 \mathrm{H}), 2.99(\mathrm{~s}, 3 \mathrm{H})$, $2.32(\mathrm{~s}, 3 \mathrm{H}) ;{ }^{13} \mathrm{C} \mathrm{NMR}\left(100 \mathrm{MHz}, \mathrm{CDCl}_{3}\right) \delta 155.6,147.4,146.3,144.0,143.1$, 134.0, 129.4, 127.9, 119.8, 112.1, 101.7, 37.2, 21.5 ppm; HRMS m/z (ESI) calcd for $\mathrm{C}_{15} \mathrm{H}_{15} \mathrm{NNaO}_{5} \mathrm{~S}(\mathrm{M}+\mathrm{Na})^{+}: 344.0563$, found 344.0570.

\section{1-((N,4-dimethylphenyl)sulfonamido)vinyl 1-methyl-1H-indazole-3-carboxylate (7f)}

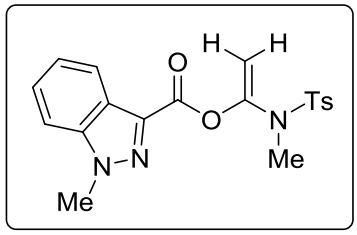

White solid, 99\% yield (76.2 mg). ${ }^{1} \mathrm{H}$ NMR (400 MHz, $\left.\mathrm{CDCl}_{3}\right) \delta 7.96$ (d, $J$ $=8.2 \mathrm{~Hz}, 1 \mathrm{H}), 7.78(\mathrm{~d}, J=8.2 \mathrm{~Hz}, 2 \mathrm{H}), 7.47(\mathrm{~d}, J=2.7 \mathrm{~Hz}, 2 \mathrm{H}), 7.34-7.27$ (m, 1H), $7.21(\mathrm{~d}, J=8.1 \mathrm{~Hz}, 2 \mathrm{H}), 5.08(\mathrm{~d}, J=2.4 \mathrm{~Hz}, 1 \mathrm{H}), 4.90(\mathrm{~d}, J=2.4$ $\mathrm{Hz}, 1 \mathrm{H}), 4.18$ (s, 3H), 3.15 (s, 3H), 2.33 (s, 3H); $\left.{ }^{13} \mathrm{C} \mathrm{NMR} \mathrm{(100} \mathrm{MHz,} \mathrm{CDCl}_{3}\right)$ $\delta 159.8,146.5,143.8,141.0,134.4,133.1,129.4,128.0,127.0,123.7,123.5$, 122.0, 109.6, 101.6, 37.2, 36.5, $21.5 \mathrm{ppm}$; HRMS m/z (ESI) calcd for $\mathrm{C}_{19} \mathrm{H}_{19} \mathrm{~N}_{3} \mathrm{NaO}_{4} \mathrm{~S}(\mathrm{M}+\mathrm{Na})^{+}$: 408.0988, found 408.0995.

1-((N,4-dimethylphenyl)sulfonamido)vinyl benzo[b]thiophene-3-carboxylate (7g)

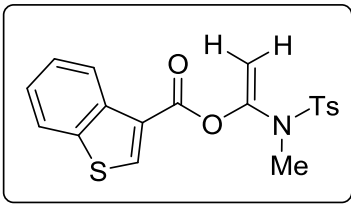

White solid, 99\% yield (77.4 mg). ${ }^{1} \mathrm{H}$ NMR (400 $\left.\mathrm{MHz}, \mathrm{CDCl}_{3}\right) \delta 7.92$ (s, $1 \mathrm{H}), 7.86(\mathrm{~d}, J=8.1 \mathrm{~Hz}, 2 \mathrm{H}), 7.76(\mathrm{~d}, J=8.2 \mathrm{~Hz}, 2 \mathrm{H}), 7.50-7.41(\mathrm{~m}, 2 \mathrm{H})$, $7.29-7.24(\mathrm{~m}, 2 \mathrm{H}), 5.09(\mathrm{~d}, J=2.5 \mathrm{~Hz}, 1 \mathrm{H}), 4.92(\mathrm{~d}, J=2.5 \mathrm{~Hz}, 1 \mathrm{H}), 3.12$ (s, 3H), $2.33(\mathrm{~s}, 3 \mathrm{H}) ;{ }^{13} \mathrm{C}$ NMR $\left(100 \mathrm{MHz}, \mathrm{CDCl}_{3}\right) \delta$ 160.0, 146.5, 144.0, 142.7, 138.4, 134.3, 132.1, 131.6, 129.6, 127.9, 127. 5, 125.7, 125.1, 122.7, 101.9, 37.1, 21.4 ppm; HRMS m/z (ESI) calcd for $\mathrm{C}_{19} \mathrm{H}_{17} \mathrm{NNaO}_{4} \mathrm{~S}_{2}(\mathrm{M}+\mathrm{Na})^{+}: 410.0491$, found 410.0497 .

\section{1-((N,4-dimethylphenyl)sulfonamido)vinyl cinnamate $(7 \mathrm{~h})$}

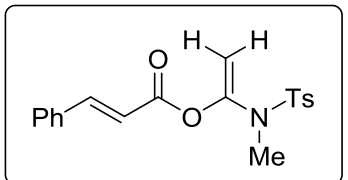

White solid, 99\% yield (70.7 mg). ${ }^{1} \mathrm{H}$ NMR $\left(400 \mathrm{MHz}, \mathrm{CDCl}_{3}\right) \delta 7.75(\mathrm{~d}, J=$ $8.3 \mathrm{~Hz}, 2 \mathrm{H}), 7.59(\mathrm{~d}, J=16.0 \mathrm{~Hz}, 1 \mathrm{H}), 7.51-7.45(\mathrm{~m}, 2 \mathrm{H}), 7.44-7.38(\mathrm{~m}$, $3 \mathrm{H}), 7.30(\mathrm{~d}, J=8.0 \mathrm{~Hz}, 2 \mathrm{H}), 6.31(\mathrm{~d}, J=16.0 \mathrm{~Hz}, 1 \mathrm{H}), 4.95(\mathrm{~d}, J=2.4 \mathrm{~Hz}$, $1 \mathrm{H}), 4.78(\mathrm{~d}, J=2.5 \mathrm{~Hz}, 1 \mathrm{H}), 3.08(\mathrm{~s}, 3 \mathrm{H}), 2.36(\mathrm{~s}, 3 \mathrm{H}) ;{ }^{13} \mathrm{C} \mathrm{NMR}(100 \mathrm{MHz}$, $\left.\mathrm{CDCl}_{3}\right) \delta 164.1,147.0,146.9,144.0,134.5,133.9,130.8,129.5,129.0,128.3,128.0,116.3,101.1,37.2$, $21.5 \mathrm{ppm}$; HRMS m/z (ESI) calcd for $\mathrm{C}_{19} \mathrm{H}_{19} \mathrm{NNaO}_{4} \mathrm{~S}(\mathrm{M}+\mathrm{Na})^{+}: 380.0927$, found 380.0927 .

\section{1-((N,4-dimethylphenyl)sulfonamido)vinyl 3-phenylpropiolate (7i)}

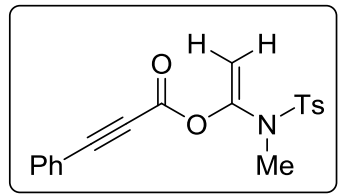

White solid, 99\% yield $(71.0 \mathrm{mg}) .{ }^{1} \mathrm{H}$ NMR $\left(400 \mathrm{MHz}, \mathrm{CDCl}_{3}\right) \delta 7.76(\mathrm{~d}, J=$ $8.2 \mathrm{~Hz}, 2 \mathrm{H}), 7.62-7.55(\mathrm{~m}, 2 \mathrm{H}), 7.49(\mathrm{t}, J=7.5 \mathrm{~Hz}, 1 \mathrm{H}), 7.40(\mathrm{t}, J=7.5 \mathrm{~Hz}$, $2 \mathrm{H}), 7.31(\mathrm{~d}, J=8.1 \mathrm{~Hz}, 2 \mathrm{H}), 4.99(\mathrm{~d}, J=2.7 \mathrm{~Hz}, 1 \mathrm{H}), 4.81(\mathrm{~d}, J=2.7 \mathrm{~Hz}$, 1H), 3.07 (s, 3H), 2.39 (s, 3H); ${ }^{13} \mathrm{C}$ NMR (100 MHz, $\left.\mathrm{CDCl}_{3}\right) \delta 151.0,146.4$, 144.1, 134.1, 133.1, 131.1, 129.6, 128.6, 128.0, 119.0, 101.8, 89.4, 79.5, 37.2, 21.5 ppm; HRMS m/z (ESI) calcd for $\mathrm{C}_{19} \mathrm{H}_{18} \mathrm{NO}_{4} \mathrm{~S}(\mathrm{M}+\mathrm{H})^{+}: 356.0957$, found 356.0958 .

\section{1-((N,4-dimethylphenyl)sulfonamido)vinyl (tert-butoxycarbonyl)-L-phenylalaninate (7j)}

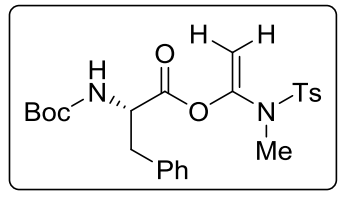

White solid, 99\% yield (94.1 mg). ${ }^{1} \mathrm{H}$ NMR (400 MHz, $\left.\mathrm{CDCl}_{3}\right) \delta 7.72(\mathrm{~d}, J=$ $8.2 \mathrm{~Hz}, 2 \mathrm{H}), 7.33-7.22(\mathrm{~m}, 5 \mathrm{H}), 7.15(\mathrm{~d}, J=7.0 \mathrm{~Hz}, 2 \mathrm{H}), 4.92-4.87(\mathrm{~m}$, 2H), $4.70(\mathrm{~s}, 1 \mathrm{H}), 4.53(\mathrm{dd}, J=13.4,6.9 \mathrm{~Hz}, 1 \mathrm{H}), 3.12(\mathrm{dd}, J=14.0,5.4 \mathrm{~Hz}$, $1 \mathrm{H}), 3.03-2.90(\mathrm{~m}, 4 \mathrm{H}), 2.42(\mathrm{~s}, 3 \mathrm{H}), 1.40(\mathrm{~s}, 9 \mathrm{H}) ;{ }^{13} \mathrm{C} \mathrm{NMR}(100 \mathrm{MHz}$, $\left.\mathrm{CDCl}_{3}\right) \delta 169.6,155.0,146.6,144.3,135.7,133.7,129.6,129.4,128.6,128.1,127.2,101.8,80.1,54.4$, 37.6, 37.0, 28.3, $21.6 \mathrm{ppm}$; HRMS m/z (ESI) calcd for $\mathrm{C}_{24} \mathrm{H}_{31} \mathrm{~N}_{2} \mathrm{O}_{6} \mathrm{~S}(\mathrm{M}+\mathrm{H})^{+}:$475.1903, found 475.1906. 


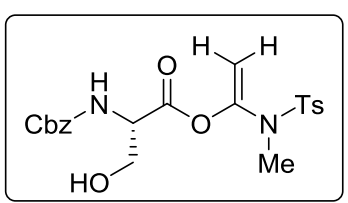

White solid, 99\% yield $(88.7 \mathrm{mg}) .{ }^{1} \mathrm{H}$ NMR $\left(400 \mathrm{MHz}, \mathrm{CDCl}_{3}\right) \delta 7.71(\mathrm{~d}, J=$ $8.2 \mathrm{~Hz}, 2 \mathrm{H}), 7.36-7.32(\mathrm{~m}, 7 \mathrm{H}), 5.84(\mathrm{~d}, J=8.4 \mathrm{~Hz}, 1 \mathrm{H}), 5.19-5.09(\mathrm{~m}$, $2 \mathrm{H}), 4.87(\mathrm{~d}, J=2.5 \mathrm{~Hz}, 1 \mathrm{H}), 4.46(\mathrm{dd}, J=15.5,5.5 \mathrm{~Hz}, 2 \mathrm{H}), 4.28-4.16(\mathrm{~m}$, $1 \mathrm{H}), 3.85(\mathrm{dd}, J=11.8,3.2 \mathrm{~Hz}, 1 \mathrm{H}), 2.98(\mathrm{~s}, 3 \mathrm{H}), 2.43(\mathrm{~s}, 3 \mathrm{H}) ;{ }^{13} \mathrm{C}$ NMR $(100$ $\left.\mathrm{MHz}, \mathrm{CDCl}_{3}\right) \delta 168.2,156.2,147.0,144.6,136.1,132.0,129.6,128.5,128.1,128.0,127.3,100.5,67.1$, 62.6, 56.3, 38.0, $21.6 \mathrm{ppm}$; HRMS m/z (ESI) calcd for $\mathrm{C}_{21} \mathrm{H}_{25} \mathrm{~N}_{2} \mathrm{O}_{7} \mathrm{~S}(\mathrm{M}+\mathrm{H})^{+}:$449.1377, found 449.1375.

\section{1-((N,4-dimethylphenyl)sulfonamido)vinyl(((9H-fluoren-9-yl)methoxy)carbonyl)-L-threoninate}

(7l)

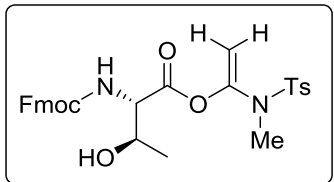

White solid, 99\% yield (108.9 mg). ${ }^{1} \mathrm{H}$ NMR (400 MHz, $\left.\mathrm{CDCl}_{3}\right) \delta 7.77-7.7$ $(\mathrm{m}, 4 \mathrm{H}), 7.64(\mathrm{t}, J=7.5 \mathrm{~Hz}, 2 \mathrm{H}), 7.43-7.37(\mathrm{~m}, 2 \mathrm{H}), 7.32(\mathrm{~d}, J=7.9 \mathrm{~Hz}$, $4 \mathrm{H}), 5.84(\mathrm{~d}, J=9.4 \mathrm{~Hz}, 1 \mathrm{H}), 4.89(\mathrm{~d}, J=2.8 \mathrm{~Hz}, 1 \mathrm{H}), 4.61-4.51(\mathrm{~m}, 1 \mathrm{H})$, $4.47-4.39(\mathrm{~m}, 4 \mathrm{H}), 4.26(\mathrm{t}, J=7.2 \mathrm{~Hz}, 1 \mathrm{H}), 2.99(\mathrm{~s}, 3 \mathrm{H}), 2.42(\mathrm{~s}, 3 \mathrm{H}), 1.30$ $(\mathrm{d}, J=6.4 \mathrm{~Hz}, 3 \mathrm{H}) ;{ }^{13} \mathrm{C} \mathrm{NMR}\left(100 \mathrm{MHz}, \mathrm{CDCl}_{3}\right) \delta 168.5,156.7,147.0,144.5,141.2,141.2,132.0,129.5$, 128.1, 127.6, 127.0, 125.1, 119.9, 100.7, 67.3, 67.0, 59.6, 47.0, 38.0, 21.5, 19.5 ppm; HRMS m/z (ESI) calcd for $\mathrm{C}_{29} \mathrm{H}_{30} \mathrm{~N}_{2} \mathrm{NaO}_{7} \mathrm{~S}(\mathrm{M}+\mathrm{Na})^{+}: 573.1666$, found 573.1669 . 


\section{Control Experiments for Two Steps Strategy and One-pot, Two-step Strategy for Amide Bond Formation}

1. Two-step Strategy (eq. 1 \& 2) and Two-step, One-pot Strategy (eq. 3) for Amide Bond Formation
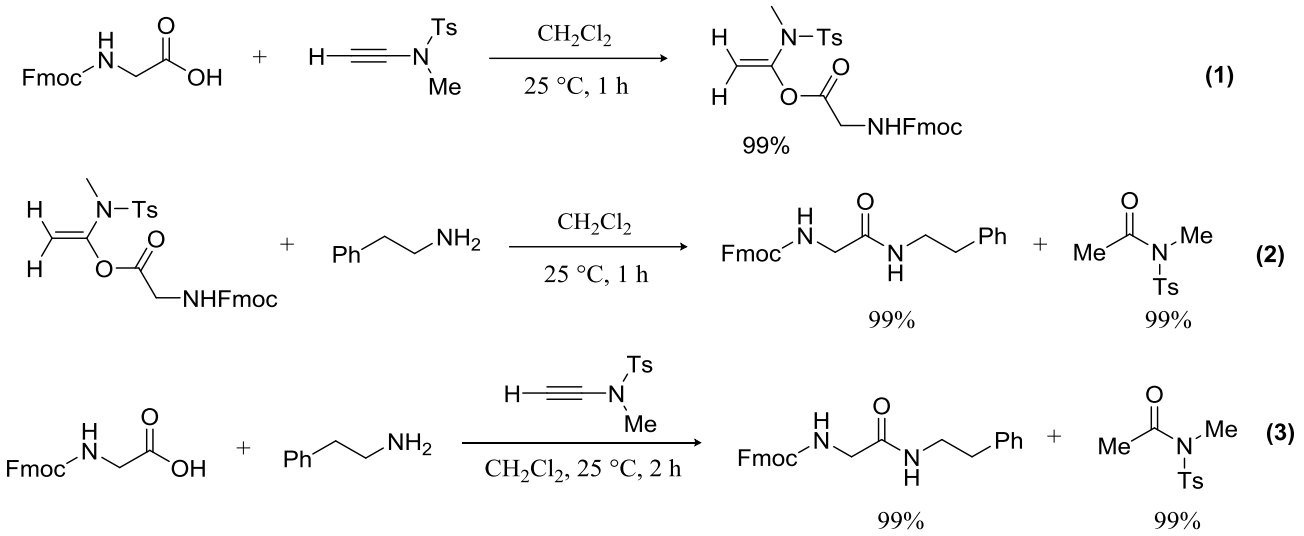

Two steps strategy: (1) A $5 \mathrm{~mL}$ round-bottomed flask was charged with $\mathrm{N}$-methyl ynetoluenesulfonamide (MYTsA) $(0.2 \mathrm{mmol}), \mathrm{CH}_{2} \mathrm{Cl}_{2}(1 \mathrm{~mL})$ and Fmoc-Gly-OH (0.2 mmol). The reaction mixture was stirred at room temperature under air until starting material was fully consumed. The reaction mixture was concentrated and purified by silica gel chromatography to afford the $\alpha$ acyloxyenamide in $99 \%$ yield. (2) To a $5 \mathrm{~mL}$ round-bottomed flask was added $\alpha$-acyloxyenamide $(0.2$ $\mathrm{mmol}), \mathrm{CH}_{2} \mathrm{Cl}_{2}(1 \mathrm{~mL})$ and 2-phenylethan-1-amine $(0.2 \mathrm{mmol})$. The reaction mixture was stirred at room temperature under air until starting material was fully consumed. The reaction mixture was concentrated and purified by silica gel chromatography to afford the respective amide in $99 \%$ yield.

Two-step, One-pot strategy: (3) A $5 \mathrm{~mL}$ round-bottomed flask was charged with $\mathrm{N}$-methyl ynetoluenesulfonamide (MYTsA) $(0.2 \mathrm{mmol}), \mathrm{CH}_{2} \mathrm{Cl}_{2}(1 \mathrm{~mL})$ and Fmoc-Gly-OH $(0.2 \mathrm{mmol})$. The reaction mixture was stirred at room temperature under air until MYTsA was fully consumed. 2phenylethan-1-amine $(0.2 \mathrm{mmol})$ was added to the above solution and reaction mixture was stirred at room temperature under air until $\alpha$-acyloxyenamide was fully consumed. The reaction mixture was concentrated and purified by silica gel chromatography to afford the respective amide in $99 \%$ yield. 
2. Solvent effect (DMF) of the Aminolysis Step
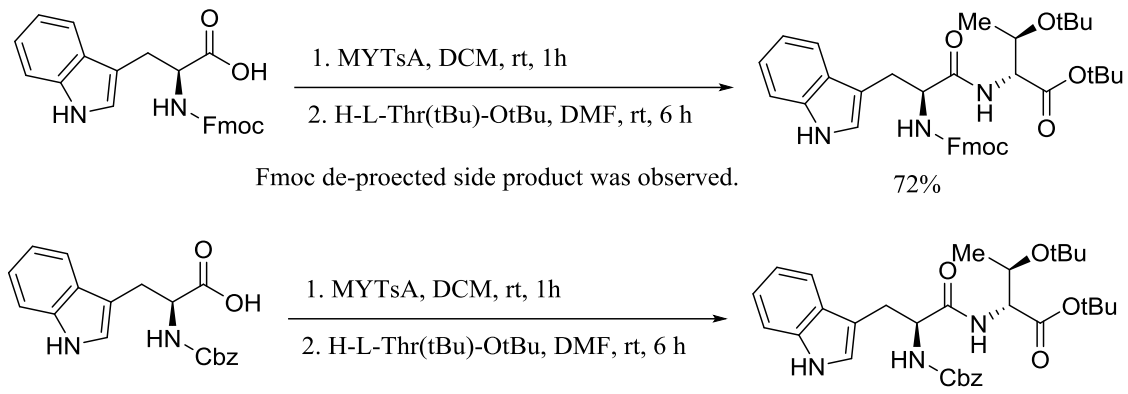

$97 \%$
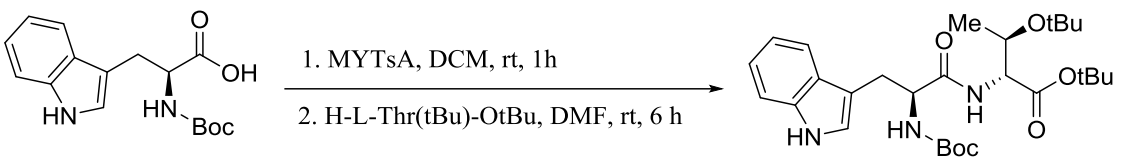

$99 \%$

A $5 \mathrm{~mL}$ round-bottomed flask was charged with $\mathrm{N}$-methyl ynetoluenesulfonamide (MYTsA) (0.2 mmol),

$\mathrm{CH}_{2} \mathrm{Cl}_{2}(1 \mathrm{~mL})$ and Fmoc-L-Trp-OH $(0.2 \mathrm{mmol})$, or Cbz-L-Trp-OH (0.2 mmol), or Boc-L-Trp-OH (0.2 mmol). The reaction mixture was stirred at room temperature under air until starting material was fully consumed. The solvent was removed under reduced pressure to afford $\alpha$-acyloxyenamide, which was charged with DMF $(1 \mathrm{~mL})$ and $\mathrm{H}-\mathrm{L}-\mathrm{Thr}(\mathrm{tBu})-\mathrm{OtBu}(0.24 \mathrm{mmol})$. The reaction mixture was stirred at room temperature under air until $\alpha$-acyloxyenamide was fully consumed. The reaction mixture was concentrated and purified by silica gel chromatography to afford the respective peptide. 
<smiles>O=C(CNC(=O)OCc1ccccc1)NCCc1ccccc1</smiles>

White solid, 99\% yield (79.2 mg). ${ }^{1} \mathrm{H}$ NMR (400 MHz, $\left.\mathrm{CDCl}_{3}\right) \delta 7.77$ (d, $J$ $=7.5 \mathrm{~Hz}, 2 \mathrm{H}), 7.58(\mathrm{~d}, J=7.3 \mathrm{~Hz}, 2 \mathrm{H}), 7.41(\mathrm{t}, J=7.4 \mathrm{~Hz}, 2 \mathrm{H}), 7.37-7.26$ (m, 4H), $7.26-7.16(\mathrm{~m}, 3 \mathrm{H}), 6.01(\mathrm{~s}, 1 \mathrm{H}), 5.45(\mathrm{~s}, 1 \mathrm{H}), 4.41(\mathrm{~d}, J=6.9 \mathrm{~Hz}$, $2 \mathrm{H}), 4.20(\mathrm{t}, J=6.8 \mathrm{~Hz}, 1 \mathrm{H}), 3.80(\mathrm{~d}, J=5.3 \mathrm{~Hz}, 2 \mathrm{H}), 3.53(\mathrm{dd}, J=13.0,6.7$ 128.5, 127.7, 127.0, 126.5, 124.9, 119.9, 67.0, 47.0, 44.4, 40.6, 35.5 ppm; HRMS m/z (ESI) calcd for $\mathrm{C}_{25} \mathrm{H}_{25} \mathrm{~N}_{2} \mathrm{O}_{3}(\mathrm{M}+\mathrm{H})^{+}:$401.1865, found 401.1863.

\section{N-methyl-N-tosylacetamide}

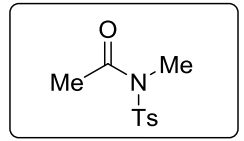

White solid, 99\% yield $(45.0 \mathrm{mg}) .{ }^{1} \mathrm{H}$ NMR $\left(400 \mathrm{MHz}, \mathrm{CDCl}_{3}\right) \delta 7.74(\mathrm{~d}, J=8.3 \mathrm{~Hz}$, 2H), 7.33 (d, $J=8.1 \mathrm{~Hz}, 2 \mathrm{H}), 3.25$ (s, 3H), 2.42 (s, 3H), 2.36 (s, 3H); ${ }^{13} \mathrm{C}$ NMR (100

$\left.\mathrm{MHz}, \mathrm{CDCl}_{3}\right) \delta 170.3,144.9,136.1,129.9,127.2,32.9,24.9,21.5 \mathrm{ppm} ; \mathrm{HRMS} \mathrm{m} / \mathrm{z}$ (ESI) calcd for $\mathrm{C}_{10} \mathrm{H}_{14} \mathrm{NO}_{3} \mathrm{~S}(\mathrm{M}+\mathrm{H})^{+}:$:228.0694, found 228.0692 .

\section{Fmoc-L-Trp-L-Thr(tBu)-OtBu}

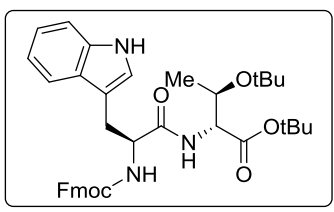

Glassy solid, $72 \%$ yield $(92.2 \mathrm{mg}) .{ }^{1} \mathrm{H}$ NMR $\left(400 \mathrm{MHz}, \mathrm{CDCl}_{3}\right) \delta 8.46(\mathrm{~s}, 1 \mathrm{H})$, $7.72(\mathrm{~d}, J=7.5 \mathrm{~Hz}, 3 \mathrm{H}), 7.51(\mathrm{t}, J=7.5 \mathrm{~Hz}, 2 \mathrm{H}), 7.42-7.30(\mathrm{~m}, 3 \mathrm{H}), 7.25(\mathrm{t}$, $J=7.3 \mathrm{~Hz}, 2 \mathrm{H}), 7.18-7.09(\mathrm{~m}, 3 \mathrm{H}), 6.59(\mathrm{~d}, J=6.5 \mathrm{~Hz}, 1 \mathrm{H}), 5.65(\mathrm{~s}, 1 \mathrm{H})$, $4.59(\mathrm{t}, J=20.0 \mathrm{~Hz}, 1 \mathrm{H}), 4.43-4.24(\mathrm{~m}, 3 \mathrm{H}), 4.21-4.07(\mathrm{~m}, 2 \mathrm{H}), 3.30-3.25$ (m, 2H), 1.45 (s, 9H), 1.09 (s, 9H), $1.02(\mathrm{~d}, J=6.0 \mathrm{~Hz}, 3 \mathrm{H}) ;{ }^{13} \mathrm{C} \mathrm{NMR}\left(100 \mathrm{MHz}, \mathrm{CDCl}_{3}\right) \delta$ 171.5, 169.4, 155.8, 143.8, 143.7, 141.1, 136.2, 127.5, 127.5, 126.9, 125.1, 125.0, 123.5, 121.9, 119.8, 119.7, 119.5, 118.7, 111.2, 81.8, 73.7, 67.0, 60.3, 58.5, 55.2, 47.0, 28.8, 28.5, 27.9, 20.5 ppm; HRMS m/z (ESI) calcd for $\mathrm{C}_{38} \mathrm{H}_{46} \mathrm{~N}_{3} \mathrm{O}_{6}(\mathrm{M}+\mathrm{H})^{+}:$640.3387, found 640.3385 .

\section{Cbz-L-Trp-L-Thr(tBu)-OtBu}

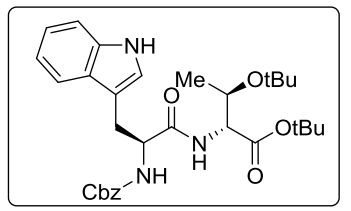

Glassy solid, 97\% yield (106.9 mg). ${ }^{1} \mathrm{H}$ NMR $\left(400 \mathrm{MHz}, \mathrm{CDCl}_{3}\right) \delta 8.33$ (s, $1 \mathrm{H}), 7.71(\mathrm{~d}, J=6.4 \mathrm{~Hz}, 1 \mathrm{H}), 7.32(\mathrm{t}, J=5.1 \mathrm{~Hz}, 6 \mathrm{H}), 7.17(\mathrm{t}, J=7.5 \mathrm{~Hz}, 1 \mathrm{H})$, $7.10(\mathrm{~d}, J=8.3 \mathrm{~Hz}, 2 \mathrm{H}), 6.55(\mathrm{~d}, J=8.6 \mathrm{~Hz}, 1 \mathrm{H}), 5.55(\mathrm{~d}, J=5.8 \mathrm{~Hz}, 1 \mathrm{H})$, $5.10(\mathrm{~s}, 2 \mathrm{H}), 4.62(\mathrm{~s}, 1 \mathrm{H}), 4.33(\mathrm{dd}, J=8.7,2.3 \mathrm{~Hz}, 1 \mathrm{H}), 4.13(\mathrm{dd}, J=13.4$, $7.8 \mathrm{~Hz}, 1 \mathrm{H}), 3.32-3.25(\mathrm{~m}, 2 \mathrm{H}), 1.46(\mathrm{~s}, 9 \mathrm{H}), 1.11(\mathrm{~s}, 9 \mathrm{H}), 1.00(\mathrm{~d}, J=5.9 \mathrm{~Hz}, 3 \mathrm{H}) ;{ }^{13} \mathrm{C}$ NMR $(100$ $\left.\mathrm{MHz}, \mathrm{CDCl}_{3}\right) \delta 171.6,169.5,156.0,136.4,136.3,128.5,128.1,128.0,127.6,123.5,122.1,119.7,118.9$, 111.2, 110.4, 81.9, 73.9, 67.1, 66.9, 58.6, 55.3, 28.8, 28.6, 28.1, 20.6 ppm; HRMS m/z (ESI) calcd for $\mathrm{C}_{31} \mathrm{H}_{42} \mathrm{~N}_{3} \mathrm{O}_{6}(\mathrm{M}+\mathrm{H})^{+}:$552.3074, found 552.3073.

\section{Boc-L-Trp-L-Thr(tBu)-OtBu}

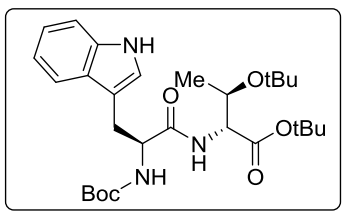

Glassy solid, 99\% yield (102.4 mg). ${ }^{1} \mathrm{H}$ NMR $\left(400 \mathrm{MHz}, \mathrm{CDCl}_{3}\right) \delta 8.38(\mathrm{~s}$, $1 \mathrm{H}), 7.67(\mathrm{~d}, J=7.7 \mathrm{~Hz}, 1 \mathrm{H}), 7.33(\mathrm{~d}, J=8.1 \mathrm{~Hz}, 1 \mathrm{H}), 7.17(\mathrm{t}, J=7.5 \mathrm{~Hz}$, $1 \mathrm{H}), 7.13-7.04(\mathrm{~m}, 2 \mathrm{H}), 6.61(\mathrm{~d}, J=8.6 \mathrm{~Hz}, 1 \mathrm{H}), 5.22(\mathrm{~d}, J=46.6 \mathrm{~Hz}, 1 \mathrm{H})$, $4.54(\mathrm{~s}, 1 \mathrm{H}), 4.31(\mathrm{dd}, J=8.7,2.4 \mathrm{~Hz}, 1 \mathrm{H}), 4.12(\mathrm{dd}, J=6.1,1.9 \mathrm{~Hz}, 1 \mathrm{H})$, $3.36-3.15(\mathrm{~m}, 2 \mathrm{H}), 1.44(\mathrm{~s}, 9 \mathrm{H}), 1.41(\mathrm{~s}, 9 \mathrm{H}), 1.12(\mathrm{~s}, 9 \mathrm{H}), 0.99(\mathrm{~d}, J=6.1 \mathrm{~Hz}, 3 \mathrm{H}) ;{ }^{13} \mathrm{C}$ NMR $(100$ $\left.\mathrm{MHz}, \mathrm{CDCl}_{3}\right) \delta 171.9,169.5,155.3,136.2,127.7,123.3,122.0,119.5,118.9,111.1,110.5,81.8,79.7$, 73.8, 67.1, 58.4, 54.8, 28.5, 28.2, 28.0, $20.4 \mathrm{ppm}$; HRMS m/z (ESI) calcd for $\mathrm{C}_{28} \mathrm{H}_{44} \mathrm{~N}_{3} \mathrm{O}_{6}(\mathrm{M}+\mathrm{H})^{+}$: 518.3230 , found 518.3230 . 


\section{Ynamide MYTsA Mediated Amide Bond Formation from Carboxylic Acids and}

\section{Amines}

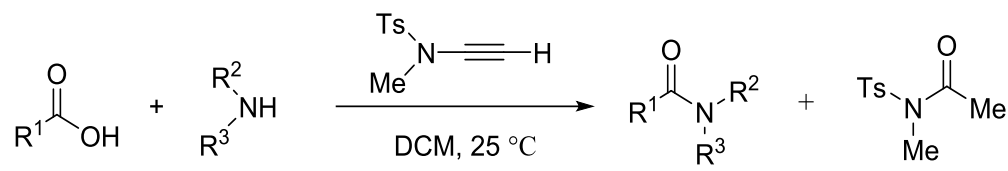

A $5 \mathrm{~mL}$ round-bottomed flask was charged with $\mathrm{N}$-methyl ynetoluenesulfonamide (MYTsA) (0.2 mmol), $\mathrm{CH}_{2} \mathrm{Cl}_{2}(1 \mathrm{~mL})$ and carboxylic acid $(0.2 \mathrm{mmol})$. The reaction mixture was stirred at room temperature under air until MYTsA was fully consumed. Amine $(0.22 \mathrm{mmol})$ was added and the reaction mixture was stirred at room temperature under air until $\alpha$-acyloxyenamide was fully consumed. The reaction mixture was concentrated and purified by silica gel chromatography to afford the respective amide.

N-phenethylacetamide (9a)

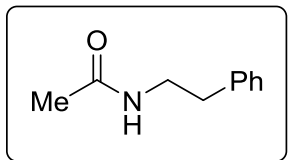

$(\mathrm{M}+\mathrm{H})^{+}:$164.1070, found 164.1067.

White solid, 98\% yield (31.9 mg). ${ }^{1} \mathrm{H}$ NMR $\left(400 \mathrm{MHz}, \mathrm{CDCl}_{3}\right) \delta 7.27(\mathrm{t}, J=7.3$ $\mathrm{Hz}, 2 \mathrm{H}), 7.21-7.16(\mathrm{~m}, 3 \mathrm{H}), 6.50(\mathrm{~s}, 1 \mathrm{H}), 3.45$ (dd, $J=13.3,7.0 \mathrm{~Hz}, 2 \mathrm{H}), 2.79$ $(\mathrm{t}, J=7.2 \mathrm{~Hz}, 2 \mathrm{H}), 1.90(\mathrm{~s}, 3 \mathrm{H}) ;{ }^{13} \mathrm{C} \mathrm{NMR}\left(100 \mathrm{MHz}, \mathrm{CDCl}_{3}\right) \delta 170.2,138.7$, $128.4,128.3,126.1,40.5,35.3,22.8$ ppm; HRMS m/z (ESI) calcd for $\mathrm{C}_{10} \mathrm{H}_{14} \mathrm{NO}$

\section{N-phenethylcinnamamide (9b)}

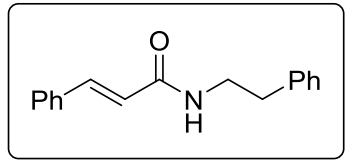

White solid, 80\% yield (40.2 mg). ${ }^{1} \mathrm{H}$ NMR (400 MHz, $\left.\mathrm{CDCl}_{3}\right) \delta 7.62(\mathrm{~d}, J=$ $15.6 \mathrm{~Hz}, 1 \mathrm{H}), 7.48-7.46(\mathrm{~m}, 2 \mathrm{H}), 7.37-7.29(\mathrm{~m}, 5 \mathrm{H}), 7.28-7.20(\mathrm{~m}, 3 \mathrm{H})$, $6.35(\mathrm{~d}, J=15.6 \mathrm{~Hz}, 1 \mathrm{H}), 5.85(\mathrm{~s}, 1 \mathrm{H}), 3.66(\mathrm{dd}, J=13.0,6.8 \mathrm{~Hz}, 2 \mathrm{H}), 2.89$ $(\mathrm{t}, J=6.9 \mathrm{~Hz}, 2 \mathrm{H}) ;{ }^{13} \mathrm{C} \mathrm{NMR}\left(100 \mathrm{MHz}, \mathrm{CDCl}_{3}\right) \delta 165.9,141.0,138.9,134.8$, 129.6, 128.8, 128.7, 128.6, 127.7, 126.5, 120.7, 40.8, 35.6 ppm; HRMS m/z (ESI) calcd for $\mathrm{C}_{17} \mathrm{H}_{18} \mathrm{NO}$ $(\mathrm{M}+\mathrm{H})^{+}:$252.1388, found 252.1385 .

\section{N-phenethylpropiolamide (9c)}

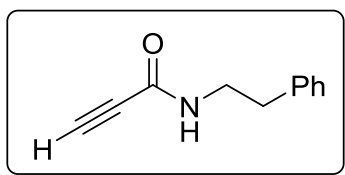

ppm; HRMS m/z (ESI) calcd for $\mathrm{C}_{11} \mathrm{H}_{12} \mathrm{NO}(\mathrm{M}+\mathrm{H})^{+}$: 174.0913, found 174.0915.

\section{N-phenethylfuran-2-carboxamide (9d)}

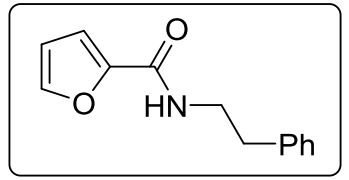

White solid, 99\% yield $(42.6 \mathrm{mg}) .{ }^{1} \mathrm{H}$ NMR $\left(400 \mathrm{MHz}, \mathrm{CDCl}_{3}\right) \delta 7.40-7.37$ $(\mathrm{m}, 1 \mathrm{H}), 7.32(\mathrm{t}, J=7.5 \mathrm{~Hz}, 2 \mathrm{H}), 7.24(\mathrm{t}, J=7.4 \mathrm{~Hz}, 3 \mathrm{H}), 7.09(\mathrm{~d}, J=3.4 \mathrm{~Hz}$, $1 \mathrm{H}), 6.59-6.27(\mathrm{~m}, 2 \mathrm{H}), 3.68(\mathrm{dd}, J=13.4,6.8 \mathrm{~Hz}, 2 \mathrm{H}), 2.91(\mathrm{t}, J=7.1 \mathrm{~Hz}$, $2 \mathrm{H}) ;{ }^{13} \mathrm{C}$ NMR $\left(100 \mathrm{MHz}, \mathrm{CDCl}_{3}\right) \delta 158.3,148.0,143.7,138.7,128.7,128.6$, 126.5, 114.0, 112.0, 40.24, $35.74 \mathrm{ppm}$; HRMS m/z (ESI) calcd for $\mathrm{C}_{13} \mathrm{H}_{14} \mathrm{NO}_{2}(\mathrm{M}+\mathrm{H})^{+}: 216.1025$, found 216.1024 .

N-phenethylbenzo[b]thiophene-3-carboxamide (9e) 


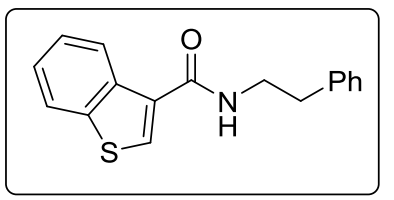

White solid, $98 \%$ yield $(55.1 \mathrm{mg}) .{ }^{1} \mathrm{H}$ NMR $\left(400 \mathrm{MHz}, \mathrm{CDCl}_{3}\right) \delta 7.90-$ $7.75(\mathrm{~m}, 2 \mathrm{H}), 7.68(\mathrm{~s}, 1 \mathrm{H}), 7.42-7.36(\mathrm{~m}, 2 \mathrm{H}), 7.34-7.30(\mathrm{~m}, 2 \mathrm{H}), 7.27$ $-7.24(\mathrm{~m}, 3 \mathrm{H}), 6.26(\mathrm{~s}, 1 \mathrm{H}), 3.71(\mathrm{dd}, J=13.0,6.8 \mathrm{~Hz}, 2 \mathrm{H}), 2.94(\mathrm{t}, J=$ $6.9 \mathrm{~Hz}, 2 \mathrm{H}) ;{ }^{13} \mathrm{C}$ NMR $\left(100 \mathrm{MHz}, \mathrm{CDCl}_{3}\right) \delta 162.2,140.7,139.0,138.7$, 138.4, 128.8, 128.7, 126.6, 126.3, 125.1, 125.0, 124.9, 122.7, 41.3, 35.7 ppm; HRMS m/z (ESI) calcd for $\mathrm{C}_{17} \mathrm{H}_{16} \mathrm{NOS}(\mathrm{M}+\mathrm{H})^{+}:$282.0947, found 282.0952 .

\section{N-(2-(1H-indol-3-yl)ethyl)-3-phenylpropiolamide (9f)}

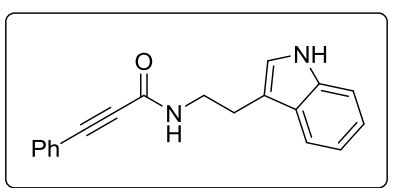

White solid, 99\% yield (57.0 mg). ${ }^{1} \mathrm{H}$ NMR $\left(400 \mathrm{MHz}, \mathrm{CDCl}_{3}\right) \delta 8.51$ (s, $1 \mathrm{H}), 7.59(\mathrm{~d}, J=7.8 \mathrm{~Hz}, 1 \mathrm{H}), 7.47-7.40(\mathrm{~m}, 2 \mathrm{H}), 7.40-7.32(\mathrm{~m}, 2 \mathrm{H})$, $7.30-7.26(\mathrm{~m}, 2 \mathrm{H}), 7.22-7.16(\mathrm{~m}, 1 \mathrm{H}), 7.14-7.08(\mathrm{~m}, 1 \mathrm{H}), 7.01(\mathrm{~d}, J$ $=2.1 \mathrm{~Hz}, 1 \mathrm{H}), 6.23(\mathrm{~s}, 1 \mathrm{H}), 3.66(\mathrm{dd}, J=12.8,6.7 \mathrm{~Hz}, 2 \mathrm{H}), 3.00(\mathrm{t}, J=$ $6.7 \mathrm{~Hz}, 2 \mathrm{H}) ;{ }^{13} \mathrm{C} \mathrm{NMR}\left(100 \mathrm{MHz}, \mathrm{CDCl}_{3}\right) \delta 153.5,136.4,132.4,129.9,128.4,127.1,122.3,122.0$, 120.0, 119.3, 118.5, 112.1, 111.4, 84.7, 83.0, 40.1, 25.0 ppm; HRMS m/z (ESI) calcd for $\mathrm{C}_{19} \mathrm{H}_{17} \mathrm{~N}_{2} \mathrm{O}$ $(\mathrm{M}+\mathrm{H})^{+}:$289.1335, found 289.1328 .

\section{4-chloro-N-(2-morpholinoethyl)benzamide (9g)}

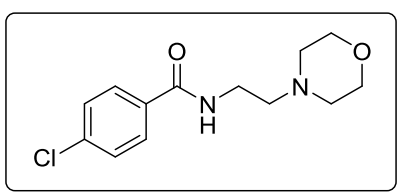

White solid, $98 \%$ yield $(57.0 \mathrm{mg}) .{ }^{1} \mathrm{H}$ NMR $\left(400 \mathrm{MHz}, \mathrm{CDCl}_{3}\right) \delta 7.76-$ $7.65(\mathrm{~m}, 2 \mathrm{H}), 7.44-7.35(\mathrm{~m}, 2 \mathrm{H}), 3.76-3.66(\mathrm{~m}, 4 \mathrm{H}), 3.52(\mathrm{dd}, J=11.2$, $5.8 \mathrm{~Hz}, 2 \mathrm{H}), 2.58(\mathrm{t}, J=6.0 \mathrm{~Hz}, 2 \mathrm{H}), 2.54-2.43(\mathrm{~m}, 4 \mathrm{H}) ;{ }^{13} \mathrm{C}$ NMR $(100$ $\left.\mathrm{MHz}, \mathrm{CDCl}_{3}\right) \delta 166.3,137.6,132.9,128.8,128.3,66.9,56.8,53.3,36.1$ ppm; HRMS m/z (ESI) calcd for $\mathrm{C}_{13} \mathrm{H}_{18} \mathrm{ClN}_{2} \mathrm{O}_{2}(\mathrm{M}+\mathrm{H})^{+}: 269.1051$, found 269.1043 .

(3r,5r,7r)-N-(tert-butyl)adamantane-1-carboxamide (9h)

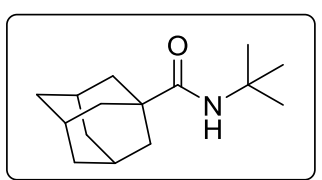

236.2002 .

White solid, 98\% yield $(46.1 \mathrm{mg}) .{ }^{1} \mathrm{H}$ NMR $\left(400 \mathrm{MHz}, \mathrm{CDCl}_{3}\right) \delta 5.35(\mathrm{~s}$, $1 \mathrm{H}), 2.01(\mathrm{~s}, 3 \mathrm{H}), 1.81-1.78(\mathrm{~m}, 6 \mathrm{H}), 1.73-1.64(\mathrm{~m}, 6 \mathrm{H}), 1.31(\mathrm{~s}, 9 \mathrm{H})$; ${ }^{13} \mathrm{C}$ NMR (100 MHz, $\left.\mathrm{CDCl}_{3}\right) \delta$ 177.6, 50.7, 41.1, 39.6, 36.7, 29.0, 28.4 ppm; HRMS m/z (ESI) calcd for $\mathrm{C}_{15} \mathrm{H}_{26} \mathrm{NO}(\mathrm{M}+\mathrm{H})^{+}$: 236.2009 , found

\section{N-cycloheptyl-3-phenylpropiolamide (9i)}

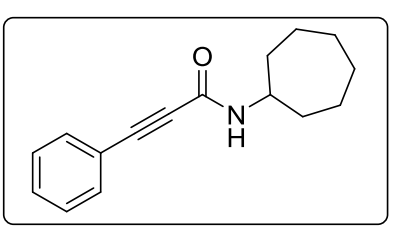

242.1539 , found 242.1531 .

White solid, 92\% yield (44.3 mg). ${ }^{1} \mathrm{H}$ NMR (400 MHz, $\left.\mathrm{CDCl}_{3}\right) \delta 7.54-$ $7.49(\mathrm{~m}, 2 \mathrm{H}), 7.42-7.29(\mathrm{~m}, 3 \mathrm{H}), 6.06(\mathrm{~d}, J=6.8 \mathrm{~Hz}, 1 \mathrm{H}), 4.08-3.97$ $(\mathrm{d}, J=4.0 \mathrm{~Hz}, 1 \mathrm{H}), 1.97$ (t, $J=11.6 \mathrm{~Hz}, 2 \mathrm{H}), 1.65-1.45(\mathrm{~m}, 10 \mathrm{H}) ;{ }^{13} \mathrm{C}$ NMR (100 MHz, $\left.\mathrm{CDCl}_{3}\right) \delta 152.4,132.5,130.0,128.6,120.5,84.1,83.6$, 51.2, 35.0, 28.1, $24.1 \mathrm{ppm}$; HRMS m/z (ESI) calcd for $\mathrm{C}_{16} \mathrm{H}_{20} \mathrm{NO}(\mathrm{M}+\mathrm{H})^{+}$:

\section{$\mathrm{N}$-(benzyloxy)-3-phenylpropiolamide (9j)}

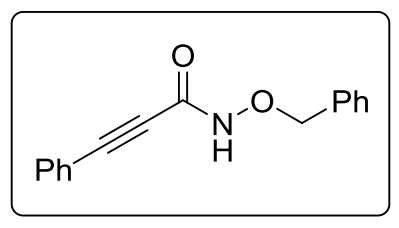

White solid, 90\% yield (45.2 mg). ${ }^{1} \mathrm{H}$ NMR (400 MHz, $\left.\mathrm{CDCl}_{3}\right) \delta 9.14$ (s, $1 \mathrm{H}), 7.49(\mathrm{~d}, J=6.1 \mathrm{~Hz}, 2 \mathrm{H}), 7.45-7.24(\mathrm{~m}, 8 \mathrm{H}), 4.97$ (s, 2H); ${ }^{13} \mathrm{C}$ NMR $\left(100 \mathrm{MHz}, \mathrm{CDCl}_{3}\right) \delta 151.9,134.8,132.5,130.3,129.2,128.7,128.5$, 128.4, 119.6, 78.0, 78.4, $29.6 \mathrm{ppm}$; HRMS m/z (ESI) calcd for $\mathrm{C}_{16} \mathrm{H}_{13} \mathrm{NNaO}_{2}(\mathrm{M}+\mathrm{Na})^{+}:$274.0838, found 274.0826.

3-phenyl-1-(pyrrolidin-1-yl)prop-2-yn-1-one (9k) 


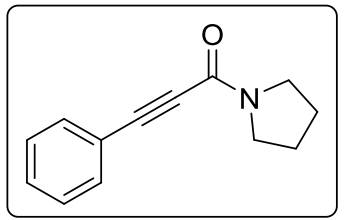

White solid, 99\% yield (39.4 mg). ${ }^{1} \mathrm{H}$ NMR $\left(400 \mathrm{MHz}, \mathrm{CDCl}_{3}\right) \delta 7.53$ (d, $J=$ $6.8 \mathrm{~Hz}, 2 \mathrm{H}), 7.41-7.32(\mathrm{~m}, 3 \mathrm{H}), 3.72(\mathrm{t}, J=6.4 \mathrm{~Hz}, 2 \mathrm{H}), 3.52(\mathrm{t}, J=6.6 \mathrm{~Hz}$, $2 \mathrm{H}), 1.99-1.91(\mathrm{~m}, 4 \mathrm{H}) ;{ }^{13} \mathrm{C}$ NMR $\left(100 \mathrm{MHz}, \mathrm{CDCl}_{3}\right) \delta 152.8,132.5,130.0$, $128.6,120.8,88.8,82.8,48.2,45.5,25.5,24.8$ ppm; HRMS m/z (ESI) calcd for $\mathrm{C}_{13} \mathrm{H}_{14} \mathrm{NO}(\mathrm{M}+\mathrm{H})^{+}: 200.1070$, found 200.1064 .

3-phenyl-1-(piperidin-1-yl)prop-2-yn-1-one (9l)

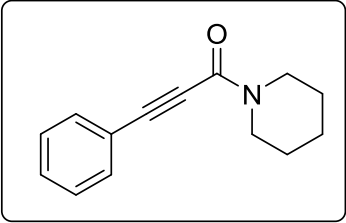

White solid, 99\% yield (42.2 mg). ${ }^{1} \mathrm{H}$ NMR (400 MHz, $\left.\mathrm{CDCl}_{3}\right) \delta 7.59-7.45$ $(\mathrm{m}, 2 \mathrm{H}), 7.43-7.28(\mathrm{~m}, 3 \mathrm{H}), 3.79-3.72(\mathrm{~m}, 2 \mathrm{H}), 3.64-3.56(\mathrm{~m}, 2 \mathrm{H}), 1.65$ $(\mathrm{dt}, J=9.9,6.3 \mathrm{~Hz}, 4 \mathrm{H}), 1.56(\mathrm{dt}, J=11.0,5.6 \mathrm{~Hz}, 2 \mathrm{H}) ;{ }^{13} \mathrm{C} \mathrm{NMR}(100 \mathrm{MHz}$, $\left.\mathrm{CDCl}_{3}\right) \delta 152.8,132.2,129.7,128.4,120.7,90.1,81.4,48.1,42.3,26.4,25.3$, $24.4 \mathrm{ppm}$; HRMS m/z (ESI) calcd for $\mathrm{C}_{14} \mathrm{H}_{16} \mathrm{NO}(\mathrm{M}+\mathrm{H})^{+}: 214.1226$, found

214.1217.

\section{N,N-diisopropyl-3-phenylpropiolamide (9m)}

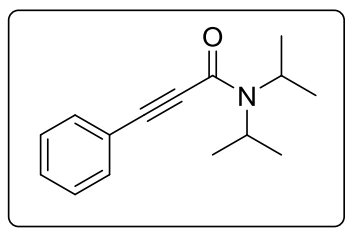

White solid, 76\% yield (34.8 mg). ${ }^{1} \mathrm{H}$ NMR (400 MHz, $\left.\mathrm{CDCl}_{3}\right) \delta 7.57-7.45$ (m, 2H), $7.41-7.29(\mathrm{~m}, 3 \mathrm{H}), 4.61-4.57(\mathrm{~m}, 1 \mathrm{H}), 3.71-3.68(\mathrm{~m}, 1 \mathrm{H}), 1.40$ $(\mathrm{d}, J=6.8 \mathrm{~Hz}, 6 \mathrm{H}), 1.29(\mathrm{~d}, J=6.8 \mathrm{~Hz}, 6 \mathrm{H}) ;{ }^{13} \mathrm{C} \mathrm{NMR}\left(100 \mathrm{MHz}, \mathrm{CDCl}_{3}\right) \delta$ 153.5, 132.1, 129.6, 128.4, 121.0, 88.4, 83.1, 50.3, 45.7, 21.0, $20.1 \mathrm{ppm}$; HRMS m/z (ESI) calcd for $\mathrm{C}_{15} \mathrm{H}_{20} \mathrm{NO}(\mathrm{M}+\mathrm{H})^{+}: 230.1539$, found 230.1535 .

\section{N-(2-hydroxyethyl)-3-phenylpropiolamide (9n)}

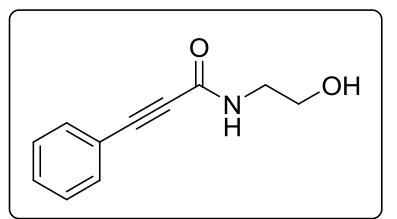

White solid, 95\% yield (35.9 mg). ${ }^{1} \mathrm{H}$ NMR $\left(400 \mathrm{MHz}, \mathrm{CDCl}_{3}\right) \delta 7.52-$ $7.46(\mathrm{~m}, 2 \mathrm{H}), 7.37(\mathrm{t}, J=4.9 \mathrm{~Hz}, 1 \mathrm{H}), 7.30(\mathrm{t}, J=7.7 \mathrm{~Hz}, 2 \mathrm{H}), 6.92(\mathrm{~s}, 1 \mathrm{H})$, $3.77-3.74(\mathrm{~m}, 2 \mathrm{H}), 3.49$ (dd, $J=10.5,5.5 \mathrm{~Hz}, 2 \mathrm{H}) ;{ }^{13} \mathrm{C}$ NMR $(100 \mathrm{MHz}$, $\left.\mathrm{CDCl}_{3}\right) \delta 154.5,132.6,130.2,128.6,120.2,85.6,82.9,61.4,42.7$ ppm; HRMS m/z (ESI) calcd for $\mathrm{C}_{11} \mathrm{H}_{12} \mathrm{NO}_{2}(\mathrm{M}+\mathrm{H})^{+}:$: 190.0863 , found 190.0862 . 
Table 2. Comparative Study of Epimerization/Racemization During Dipeptide Synthesis with Fmoc-LSer(tBu)-L-Leu-OtBu as the Model

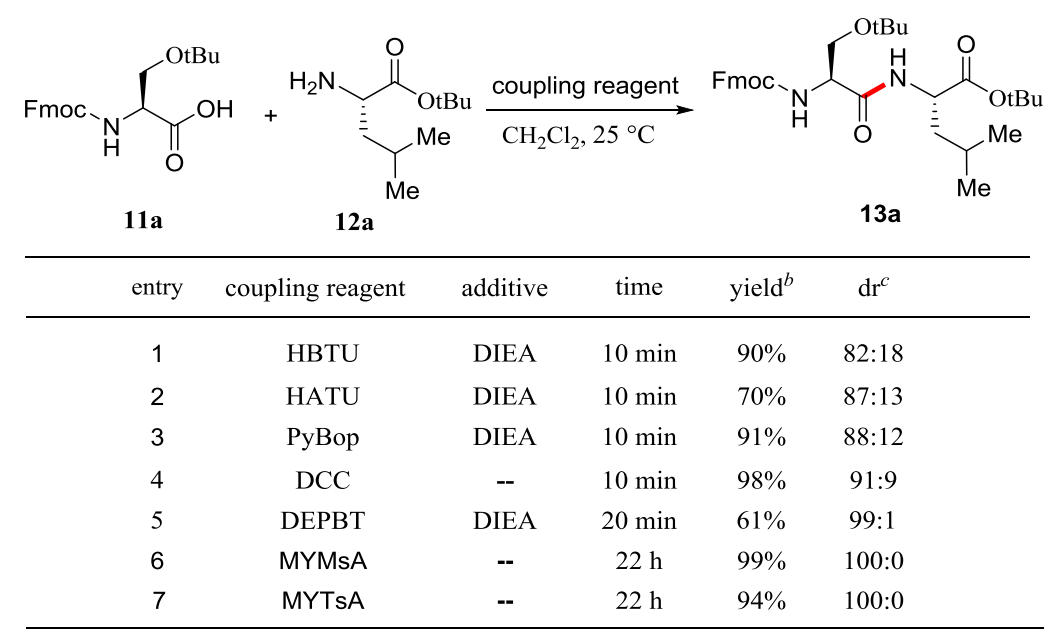

${ }^{a}$ Reaction conditions: 11a $(0.2 \mathrm{mmol}), \mathbf{1 2 a}(0.22 \mathrm{mmol})$, coupling reagent $(0.22 \mathrm{mmol})$, DIEA $(0.4 \mathrm{mmol})$. ${ }^{b}$ Isolated yield. ${ }^{c}$ Determined by HPLC analysis.

HBTU, HATU, PyBop, DEPBT: A $5 \mathrm{~mL}$ round-bottomed flask was charged with the respective coupling reagent $(0.22 \mathrm{mmol}), \mathrm{CH}_{2} \mathrm{Cl}_{2}(1 \mathrm{~mL})$, Fmoc-L-Ser(tBu)-OH (0.2 mmol), H-L-Leu-OtBu $(0.22$ mmol) and DIEA $(0.4 \mathrm{mmol})$. The reaction mixture was stirred at room temperature under air until starting material was fully consumed. The diastereomeric ratio (dr) was determined by chiral HPLC with chiral IC column with hexane and $i-\mathrm{PrOH}$ as eluent. The reaction mixture was concentrated and purified by silica gel chromatography to afford the respective peptide.

DCC: A $5 \mathrm{~mL}$ round-bottomed flask was charged with coupling reagent $(0.22 \mathrm{mmol}), \mathrm{CH}_{2} \mathrm{Cl}_{2}(1 \mathrm{~mL})$, Fmoc-L-Ser(tBu)-OH $(0.2 \mathrm{mmol})$ and H-L-Leu-OtBu(0.22 mmol). The reaction mixture was stirred at room temperature under air until starting material was fully consumed by TLC. The diastereomeric ratio (dr) was determined by chiral HPLC with chiral IC column with hexane and $i$-PrOH as eluent. The reaction mixture was concentrated and purified by silica gel chromatography to afford the respective peptide.

MYMsA, MYTsA: A $5 \mathrm{~mL}$ round-bottomed flask was charged with coupling reagent (0.22 mmol), $\mathrm{CH}_{2} \mathrm{Cl}_{2}(1 \mathrm{~mL})$ and Fmoc-L-Ser(tBu)-OH $(0.2 \mathrm{mmol})$. The reaction mixture was stirred at room temperature under air until starting material was fully consumed. H-L-Leu-OtBu( $0.22 \mathrm{mmol})$ was added to the above solution and the reaction mixture was stirred at room temperature under air until $\alpha$ acyloxyenamide was fully consumed. The diastereomeric ratio (dr) was determined by chiral HPLC with chiral IC column with hexane and $i-\mathrm{PrOH}$ as eluent. The reaction mixture was concentrated and purified by silica gel chromatography to afford the respective peptide. 


\section{Copies of HPLC Chromatograms}

1:1 mixture of Fmoc-L-Ser(tBu)-L-Leu-OtBu and Fmoc-D-Ser(tBu)-L-Leu-OtBu

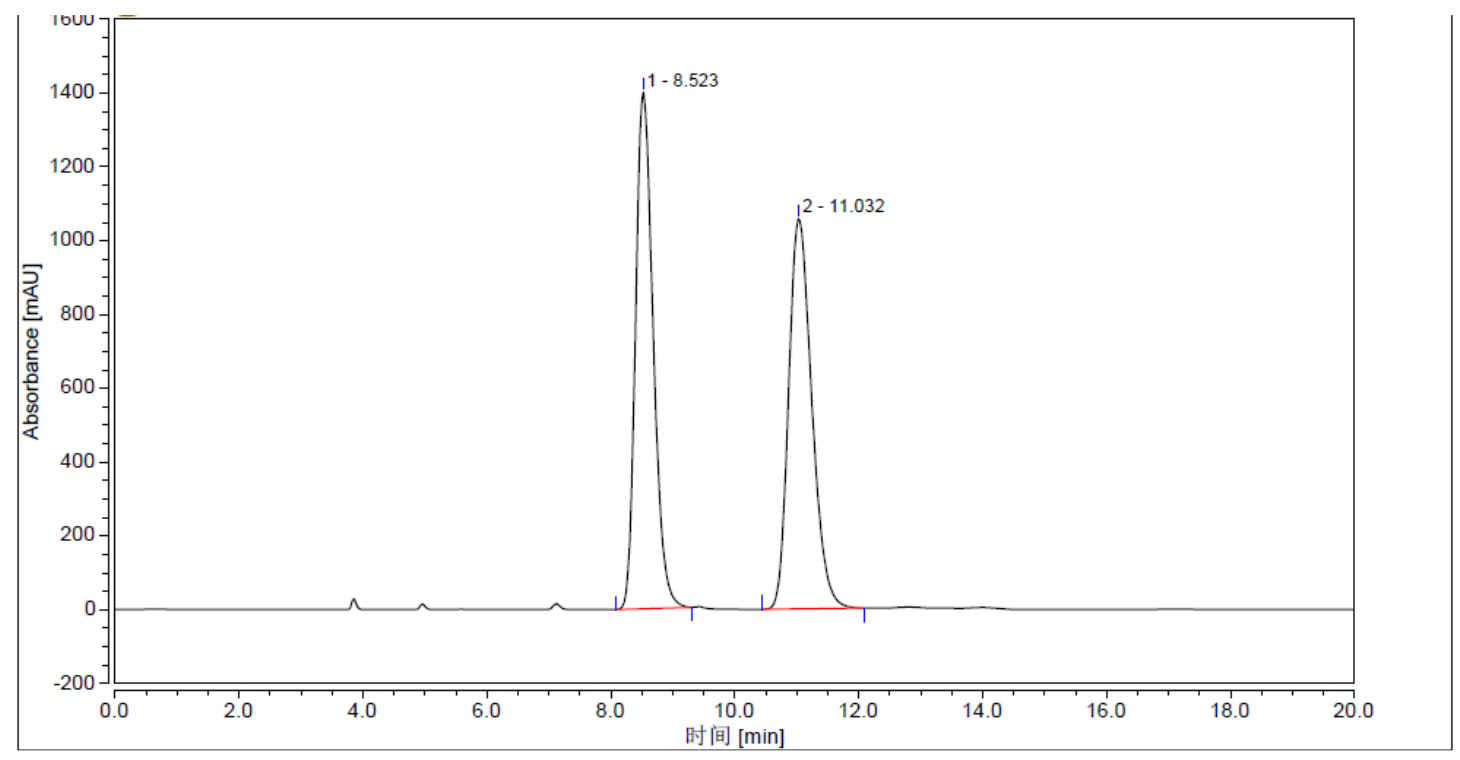

Peak Info

\begin{tabular}{|l|l|l|l|l|}
\hline Peak No & \% Area & Area & RT $(\min )$ & Height $(\mathrm{mAV})$ \\
\hline 1 & 50.22 & 460.450 & 8.523 & 1400.183 \\
\hline 2 & 49.78 & 456.369 & 11.032 & 1058.451 \\
\hline Total: & 100 & 916.818 & & 2458.635 \\
\hline
\end{tabular}

HBTU: mixture of Fmoc-D-Ser(tBu)-L-Leu-OtBu (minor) and Fmoc-L-Ser(tBu)-L-Leu-OtBu (major)

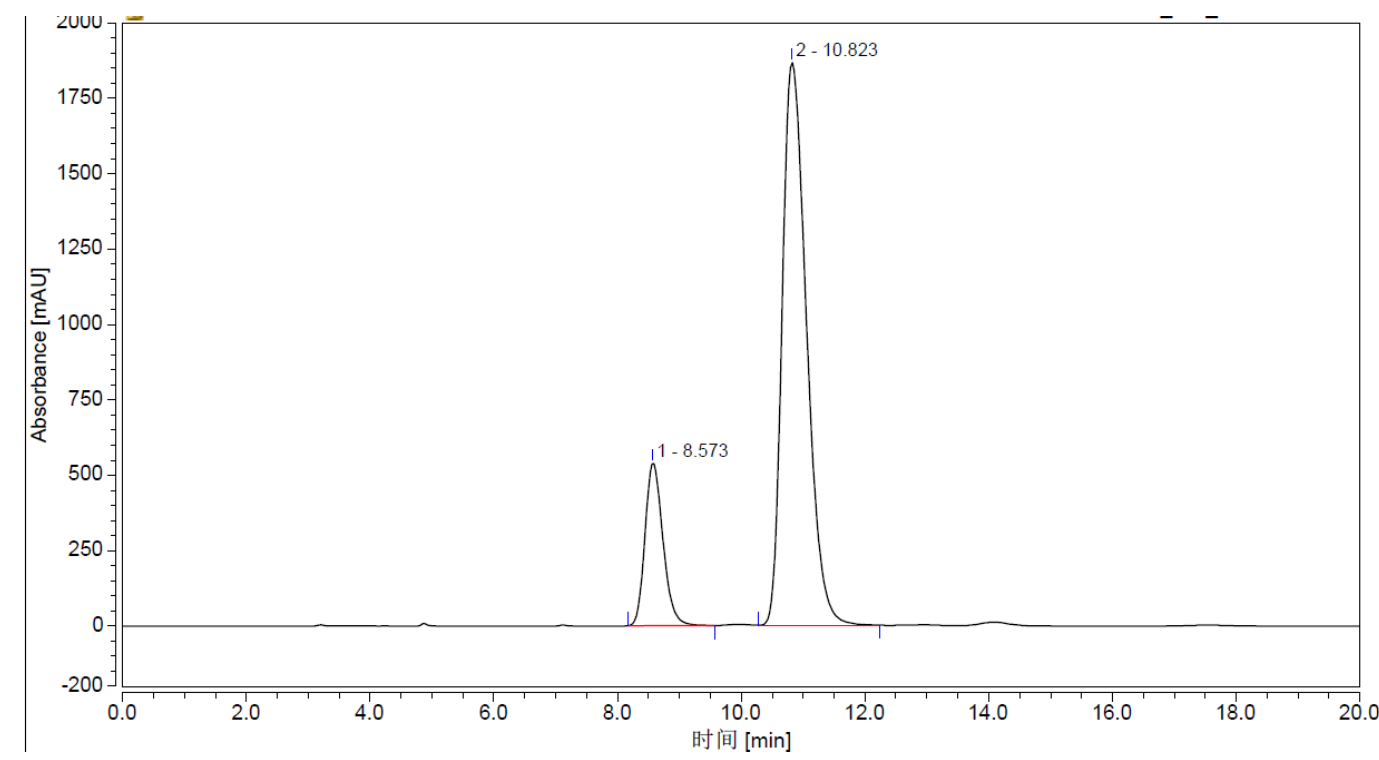

Peak Info

\begin{tabular}{|l|l|l|l|l|}
\hline Peak No & \% Area & Area & RT $(\min )$ & Height $(\mathrm{mAV})$ \\
\hline 1 & 17.63 & 181.403 & 8.573 & 540.029 \\
\hline 2 & 82.37 & 847.365 & 10.823 & 1885.834 \\
\hline Total: & 100 & 1028.768 & & 2405.862 \\
\hline
\end{tabular}


HATU: mixture of Fmoc-D-Ser(tBu)-L-Leu-OtBu (minor) and Fmoc-L-Ser(tBu)-L-Leu-OtBu (major)

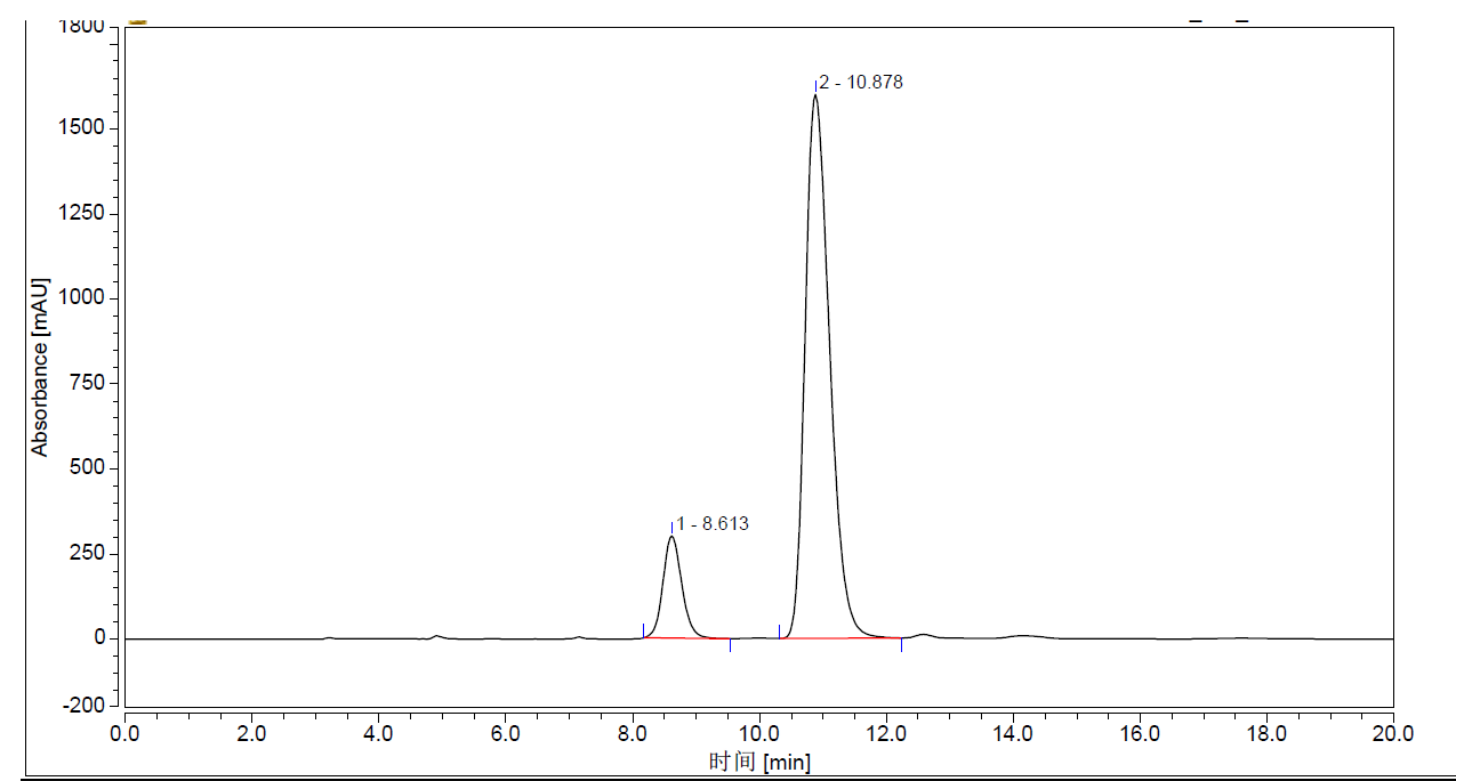

Peak Info

\begin{tabular}{|l|l|l|l|l|}
\hline Peak No & \% Area & Area & RT $(\mathrm{min})$ & Height $(\mathrm{mAV})$ \\
\hline 1 & 12.59 & 103.111 & 8.613 & 301.236 \\
\hline 2 & 87.41 & 716.138 & 10.878 & 1599.708 \\
\hline Total: & 100 & 819.248 & & 1900.944 \\
\hline
\end{tabular}

PyBop: mixture of Fmoc-D-Ser(tBu)-L-Leu-OtBu (minor) and Fmoc-L-Ser(tBu)-L-Leu-OtBu (major)

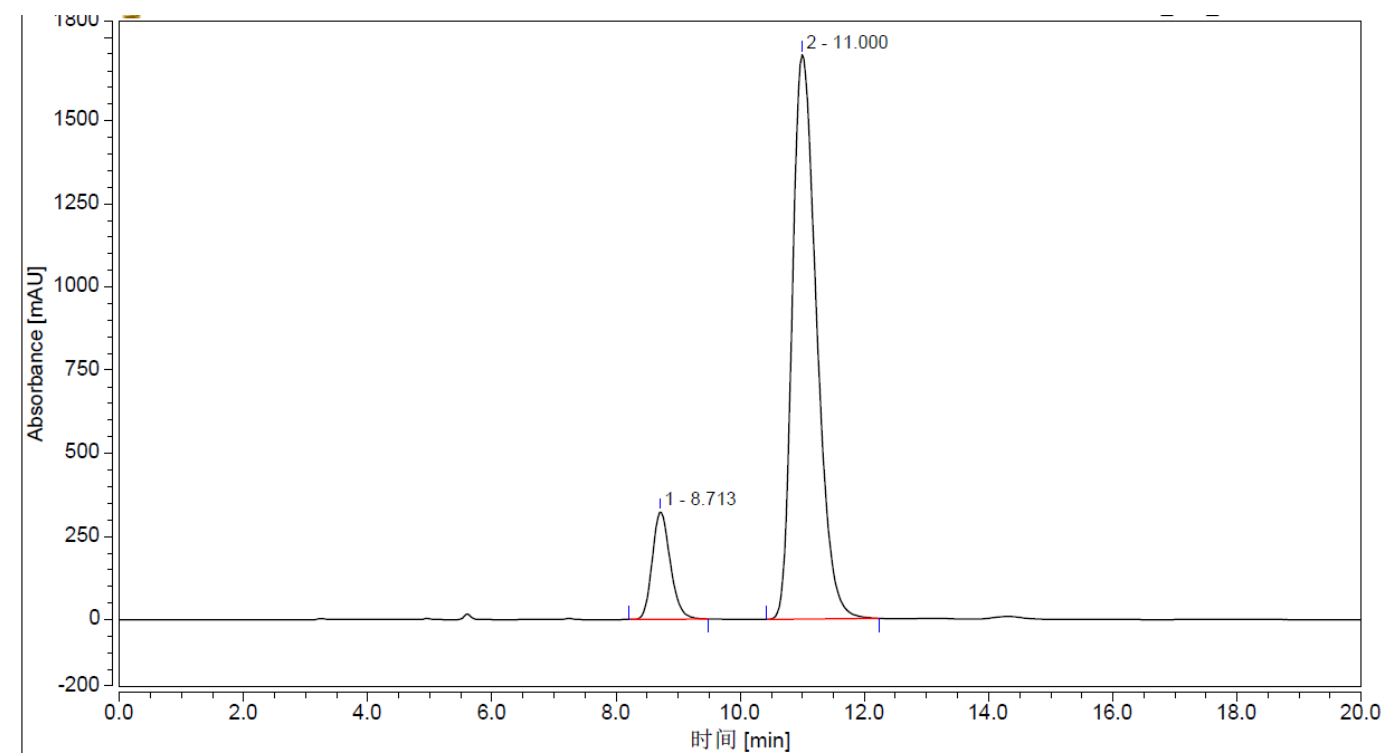

Peak Info

\begin{tabular}{|l|l|l|l|l|}
\hline Peak No & \% Area & Area & RT $(\min )$ & Height $(\mathrm{mAV})$ \\
\hline 1 & 12.41 & 108.734 & 8.713 & 323.447 \\
\hline 2 & 87.59 & 767.295 & 11.000 & 1697.579 \\
\hline Total: & 100 & 876.029 & & 2021.026 \\
\hline
\end{tabular}


DEPBT: mixture of Fmoc-D-Ser(tBu)-L-Leu-OtBu (minor) and Fmoc-L-Ser(tBu)-L-Leu-OtBu (major)

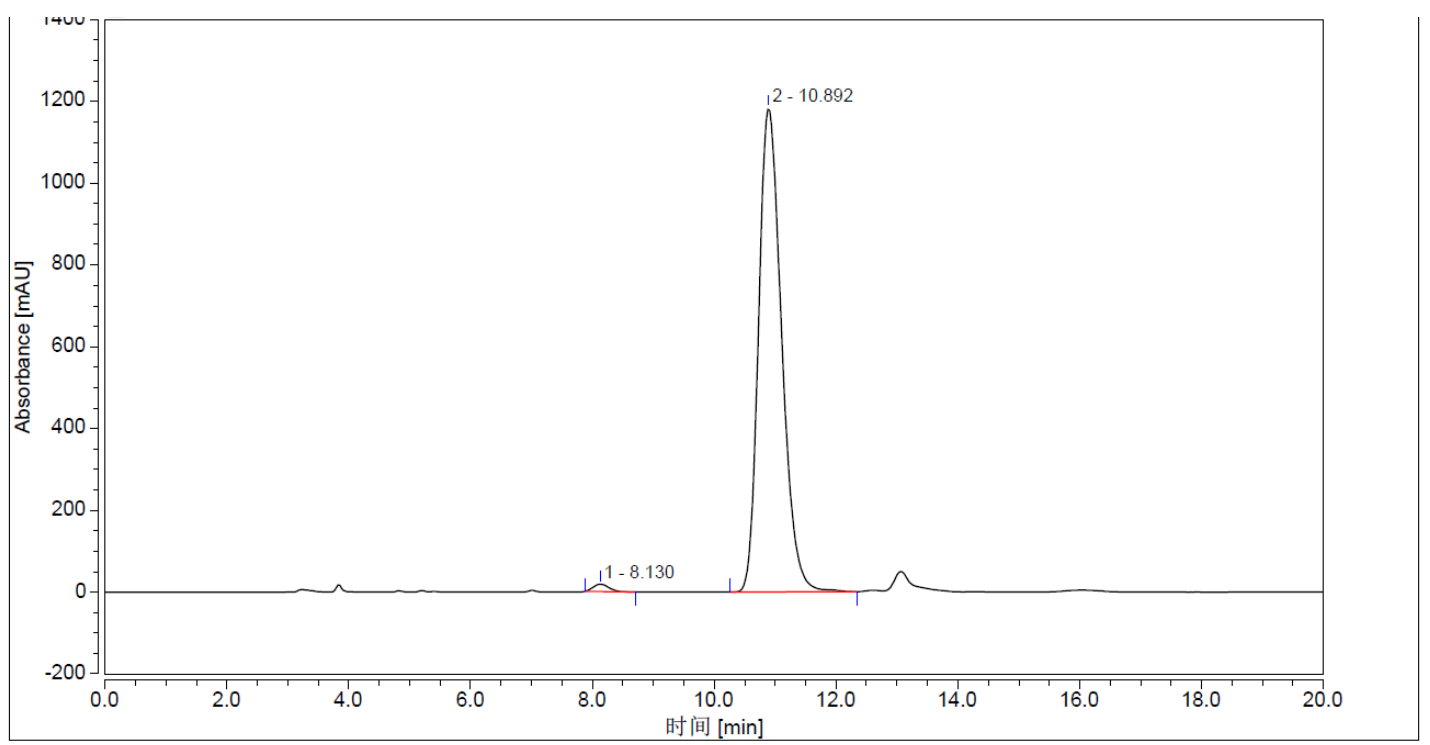

Peak Info

\begin{tabular}{|l|l|l|l|l|}
\hline Peak No & \% Area & Area & RT (min) & Height (mAV) \\
\hline 1 & 1.04 & 5.457 & 8.130 & 18.390 \\
\hline 2 & 98.96 & 516.990 & 10.892 & 1182.739 \\
\hline Total: & 100 & 522.446 & & 1201.129 \\
\hline
\end{tabular}

DCC: mixture of Fmoc-D-Ser(tBu)-L-Leu-OtBu (minor) and Fmoc-L-Ser(tBu)-L-Leu-OtBu (major)

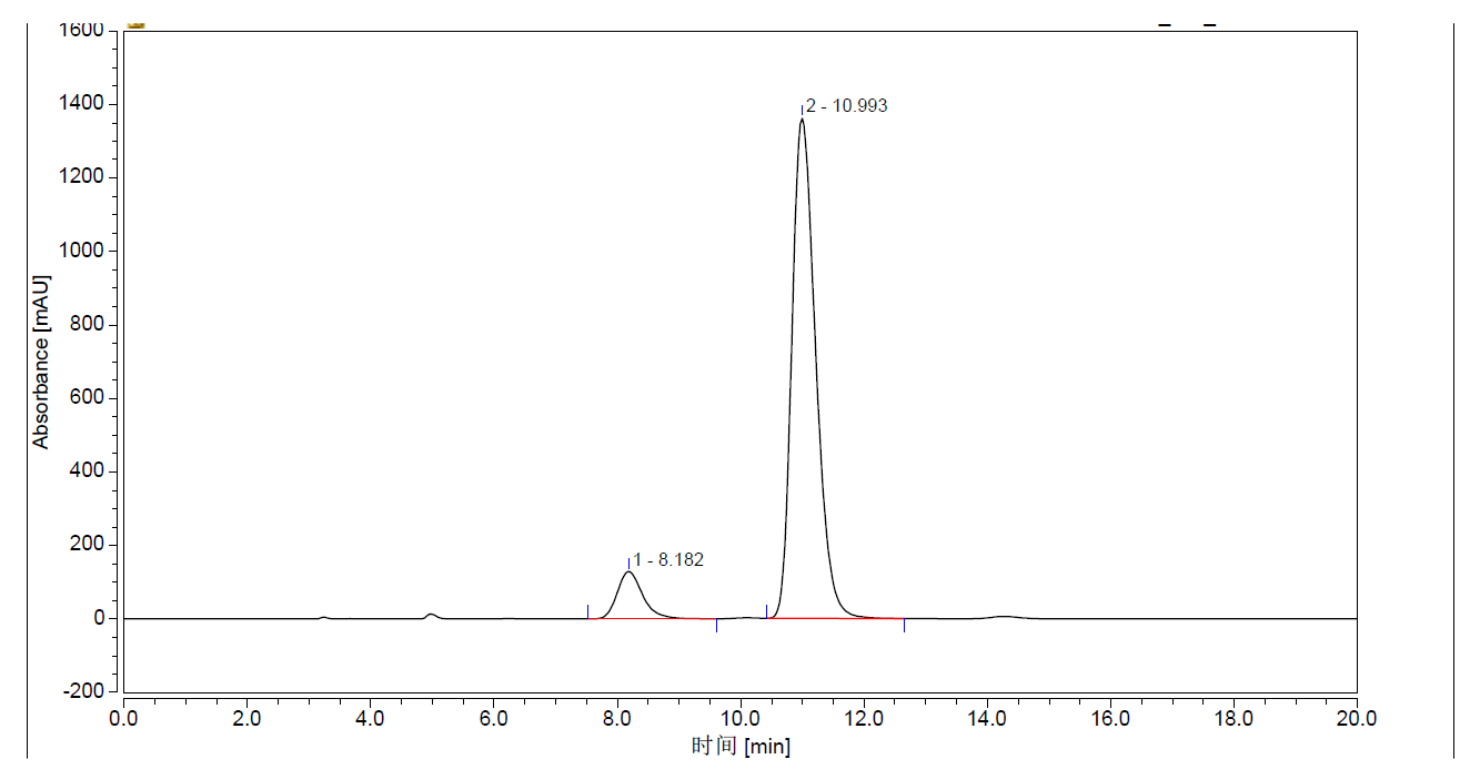

Peak Info

\begin{tabular}{|l|l|l|l|l|}
\hline Peak No & \% Area & Area & RT (min) & Height (mAV) \\
\hline 1 & 9.22 & 61.706 & 8.182 & 129.034 \\
\hline 2 & 90.78 & 607.530 & 10.993 & 1361.111 \\
\hline Total: & 100 & 669.236 & & 1490.145 \\
\hline
\end{tabular}


MYMsA: mixture of Fmoc-D-Ser(tBu)-L-Leu-OtBu (minor) and Fmoc-L-Ser(tBu)-L-Leu-OtBu (major)

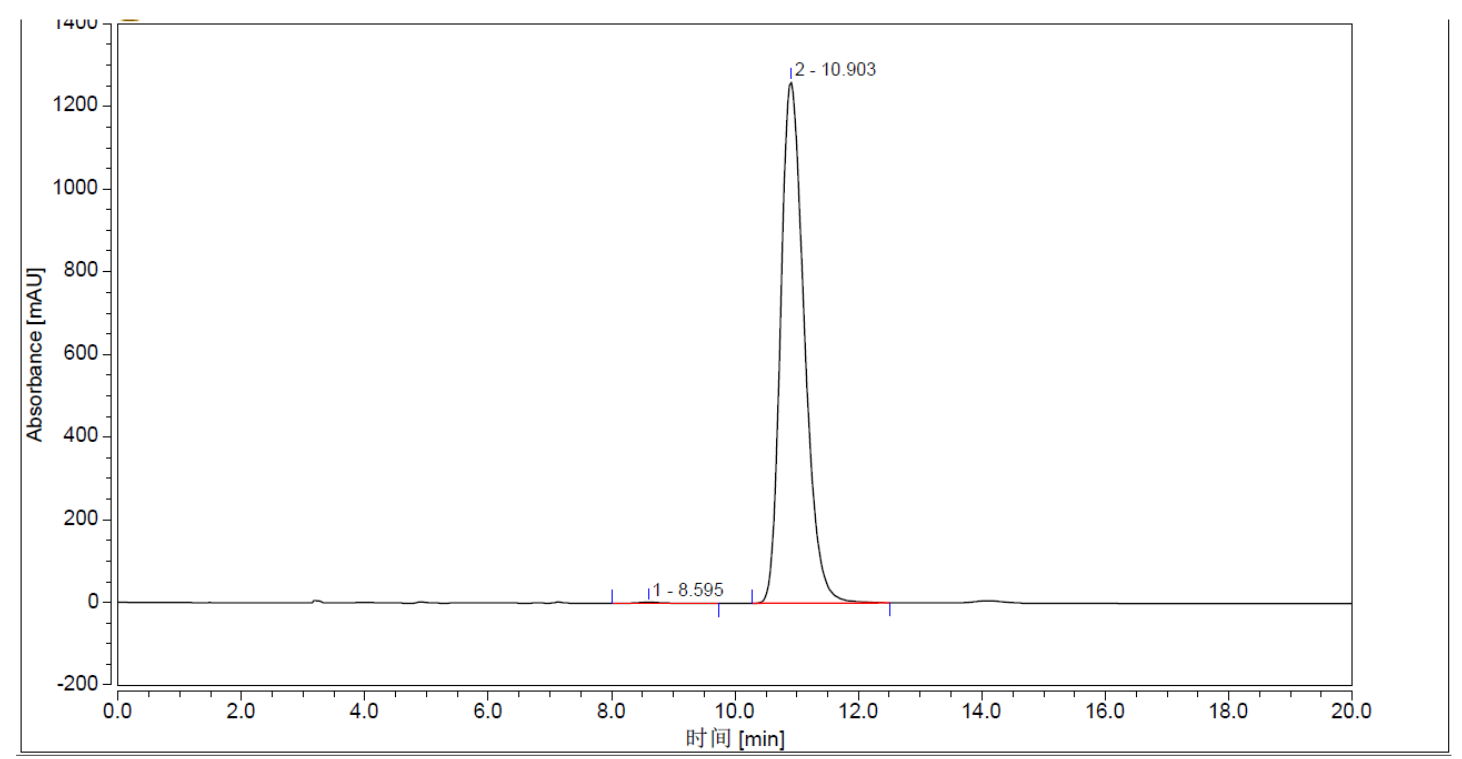

Peak Info

\begin{tabular}{|l|l|l|l|l|}
\hline Peak No & \% Area & Area & RT (min) & Height (mAV) \\
\hline 1 & 0.16 & 0.872 & 8.595 & 2.292 \\
\hline 2 & 99.84 & 559.171 & 10.903 & 1261.108 \\
\hline Total: & 100 & 560.043 & & 1263.399 \\
\hline
\end{tabular}

MYTsA: mixture of Fmoc-D-Ser(tBu)-L-Leu-OtBu (minor) and Fmoc-L-Ser(tBu)-L-Leu-OtBu(major)

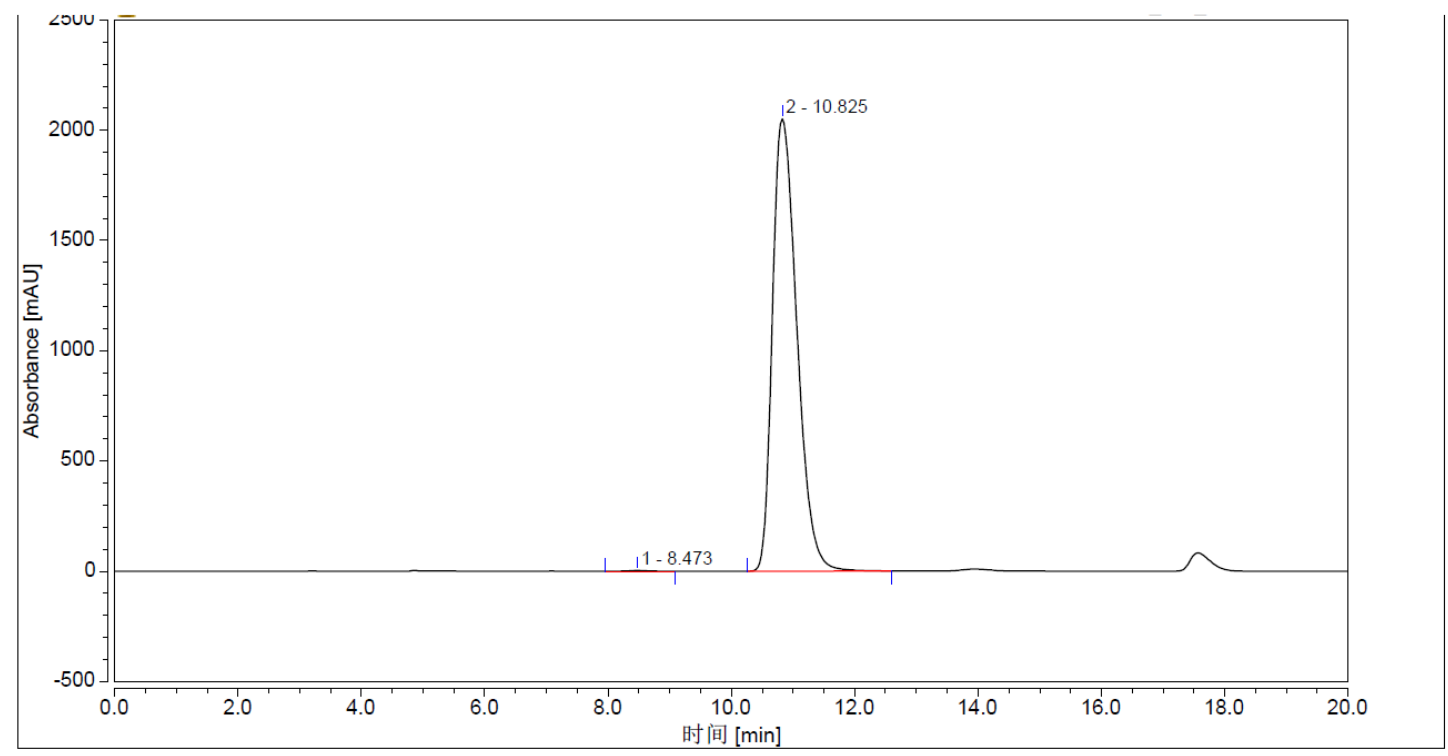

Peak Info

\begin{tabular}{|l|l|l|l|l|}
\hline Peak No & \% Area & Area & RT (min) & Height (mAV) \\
\hline 1 & 0.13 & 1.217 & 8.473 & 3.666 \\
\hline 2 & 99.87 & 940.820 & 10.825 & 2051.845 \\
\hline Total: & 100 & 942.037 & & 2055.511 \\
\hline
\end{tabular}




\section{Comparative Study of Epimerization/Racemization During Dipeptide Synthesis with Fmoc-L-Phg-L-Leu-OtBu as the Model}

The control experiment with H-L-phenylglycine, a non-natural amino acid which is extremely prone to epimerize/racemize during peptide synthesis, was used as a model to test the limit of ynamide coupling reagent were performed. According to the experimental results (Table 3), the ynamide coupling reagent MYMsA still be the best one among the tested coupling reagents albeit small amount of epimerization was observed. To further probe the origin of the epimerization/racemization of phenylglycine, a control experiment regarding the racemization of phenylglycine during hydroacyloxylation was performed. As shown in Scheme 1, no racemization was observed during hydroacyloxylation of phenylglycine. Such result clearly demonstrated that the epimerization/racemization of phenylglycine in peptide synthesis was not occurred in the activation step but in the subsequent aminolysis of $\alpha$-acyloxyenamide. We believed that such epimerization/racemization could be suppressed by careful optimization the reaction conditions of the aminolysis step.

Table 3. Comparative Study of Epimerization/Racemization During Dipeptide Synthesis with Fmoc-LPhg-L-Leu-OtBu as the Model

12a
1

${ }^{a}$ Reaction conditions: $11 x(0.2 \mathrm{mmol}), \mathbf{1 2 a}(0.22 \mathrm{mmol})$, coupling reagent $(0.22 \mathrm{mmol}), \mathrm{DIEA}(0.4 \mathrm{mmol})$.

${ }^{b}$ Isolated yield. ${ }^{c}$ Determined by HPLC analysis.

Scheme 1. Study on the racemization of phenylglycine during hydroacyloxylation with MYMsA

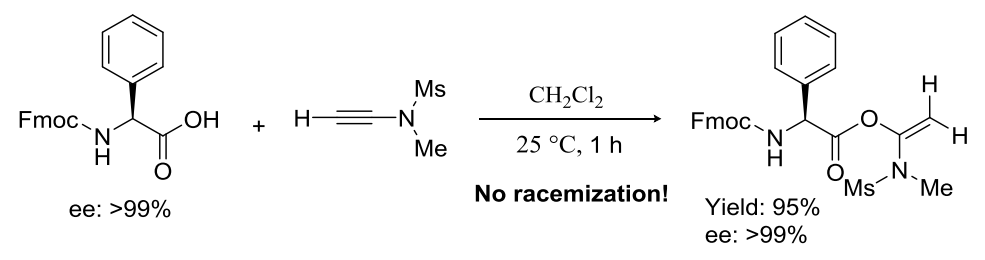


HBTU, HATU, PyBop, DEPBT: A $5 \mathrm{~mL}$ round-bottomed flask was charged with the respective coupling reagent (0.22 mmol), $\mathrm{CH}_{2} \mathrm{Cl}_{2}(1 \mathrm{~mL})$, Fmoc-L-Phg-OH (0.2 mmol), H-L-Leu-OtBu (0.22 mmol) and DIEA $(0.4 \mathrm{mmol})$. The reaction mixture was stirred at room temperature under air until starting material was fully consumed. The diastereomeric ratio (dr) was determined by chiral HPLC with chiral IC columncolumn with hexane and $i-\mathrm{PrOH}$ as eluent. The reaction mixture was concentrated and purified by silica gel chromatography to afford the respective peptide.

MYMsA, MYTsA: A $5 \mathrm{~mL}$ round-bottomed flask was charged with coupling reagent $(0.22 \mathrm{mmol})$, $\mathrm{CH}_{2} \mathrm{Cl}_{2}(1 \mathrm{~mL})$ and Fmoc-L-Phg-OH $(0.2 \mathrm{mmol})$. The reaction mixture was stirred at room temperature under air until starting material was fully consumed. H-L-Leu-OtBu( $0.22 \mathrm{mmol})$ was added to the above solution and the reaction mixture was stirred at room temperature under air until $\alpha$-acyloxyenamide was fully consumed. The diastereomeric ratio (dr) was determined by chiral HPLC with chiral IC column with hexane and $i$-PrOH as eluent. The reaction mixture was concentrated and purified by silica gel chromatography to afford the respective peptide. 


\section{Fmoc-L-Phg-L-Leu-OtBu (13x)}<smiles>CCOC(=O)[C@H](CC(C)C)NC(=O)C(NC(C)C)c1ccccc1</smiles>

White solid, 97\% yield (105.3 mg). ${ }^{1} \mathrm{H}$ NMR $\left(400 \mathrm{MHz}, \mathrm{CDCl}_{3}\right) \delta$ $7.75(\mathrm{~d}, J=7.6 \mathrm{~Hz}, 2 \mathrm{H}), 7.58(\mathrm{~s}, 2 \mathrm{H}), 7.46-7.27(\mathrm{~m}, 9 \mathrm{H}), 6.20(\mathrm{~d}$, $J=21.8 \mathrm{~Hz}, 2 \mathrm{H}), 5.29(\mathrm{~s}, 1 \mathrm{H}), 4.47(\mathrm{dd}, J=13.8,8.0 \mathrm{~Hz}, 1 \mathrm{H}), 4.36$ (d, $J=6.9 \mathrm{~Hz}, 2 \mathrm{H}), 4.19(\mathrm{t}, J=6.7 \mathrm{~Hz}, 1 \mathrm{H}), 1.72-1.48(\mathrm{~m}, 3 \mathrm{H})$, $1.35(\mathrm{~s}, 9 \mathrm{H}), 0.94(\mathrm{~d}, J=6.3 \mathrm{~Hz}, 6 \mathrm{H}) ;{ }^{13} \mathrm{C}$ NMR $\left(100 \mathrm{MHz}, \mathrm{CDCl}_{3}\right)$ $\delta 171.1,169.3,155.7,143.9,143.8,141.3,137.9,129.1,128.6$, $127.7,127.3,127.1,125.2,112.0,82.0,67.2,58.8,52.0,47.2,41.8$, 27.9, 24.9, 22.7, $22.2 \mathrm{ppm}$; HRMS m/z (ESI) calcd for $\mathrm{C}_{33} \mathrm{H}_{38} \mathrm{~N}_{2} \mathrm{NaO}_{5}(\mathrm{M}+\mathrm{Na})^{+}: 565.2673$, found 565.2679 .

1-(N-methylmethylsulfonamido)vinyl (R)-2-((((9H-fluoren-9-yl)methoxy)carbonyl)amino)-2-

phenylacetate<smiles>C=C(OC(=O)C(NC(C)C)c1ccccc1)N(C)C</smiles>

White solid, 95\% yield (96.2 mg). ${ }^{1} \mathrm{H}$ NMR (400 MHz, $\left.\mathrm{CDCl}_{3}\right) \delta 7.76$ $(\mathrm{d}, J=7.5 \mathrm{~Hz}, 2 \mathrm{H}), 7.59(\mathrm{~d}, J=7.3 \mathrm{~Hz}, 2 \mathrm{H}), 7.42-7.38(\mathrm{~m}, 7 \mathrm{H}), 7.30$ (t, $J=6.9 \mathrm{~Hz}, 2 \mathrm{H}), 5.86(\mathrm{~d}, J=6.7 \mathrm{~Hz}, 1 \mathrm{H}), 5.44(\mathrm{~d}, J=6.7 \mathrm{~Hz}, 1 \mathrm{H})$, $5.09(\mathrm{~s}, 1 \mathrm{H}), 4.88(\mathrm{~s}, 1 \mathrm{H}), 4.42(\mathrm{~d}, J=6.5 \mathrm{~Hz}, 2 \mathrm{H}), 4.21(\mathrm{t}, J=6.7 \mathrm{~Hz}$, $1 \mathrm{H}), 2.89(\mathrm{~s}, 3 \mathrm{H}), 2.72(\mathrm{~s}, 3 \mathrm{H}) ;{ }^{13} \mathrm{C} \mathrm{NMR}\left(100 \mathrm{MHz}, \mathrm{CDCl}_{3}\right) \delta 168.7$, $155.4,145.2,143.6,143.5,141.2,135.1,129.2,127.7,127.4,127.0$, 124.9, 119.9, 101.5, 67.3, 58.2, 47.0, 37.6, $35.3 \mathrm{ppm}$; HRMS m/z (ESI) calcd for $\mathrm{C}_{27} \mathrm{H}_{26} \mathrm{~N}_{2} \mathrm{NaO}_{6} \mathrm{~S}$ $(\mathrm{M}+\mathrm{Na})^{+}:$529.1404, found 529.1395. 
1:1 mixture of Fmoc-L-Phg -L-Leu-OtBu and Fmoc-D-Phg -L-Leu-OtBu

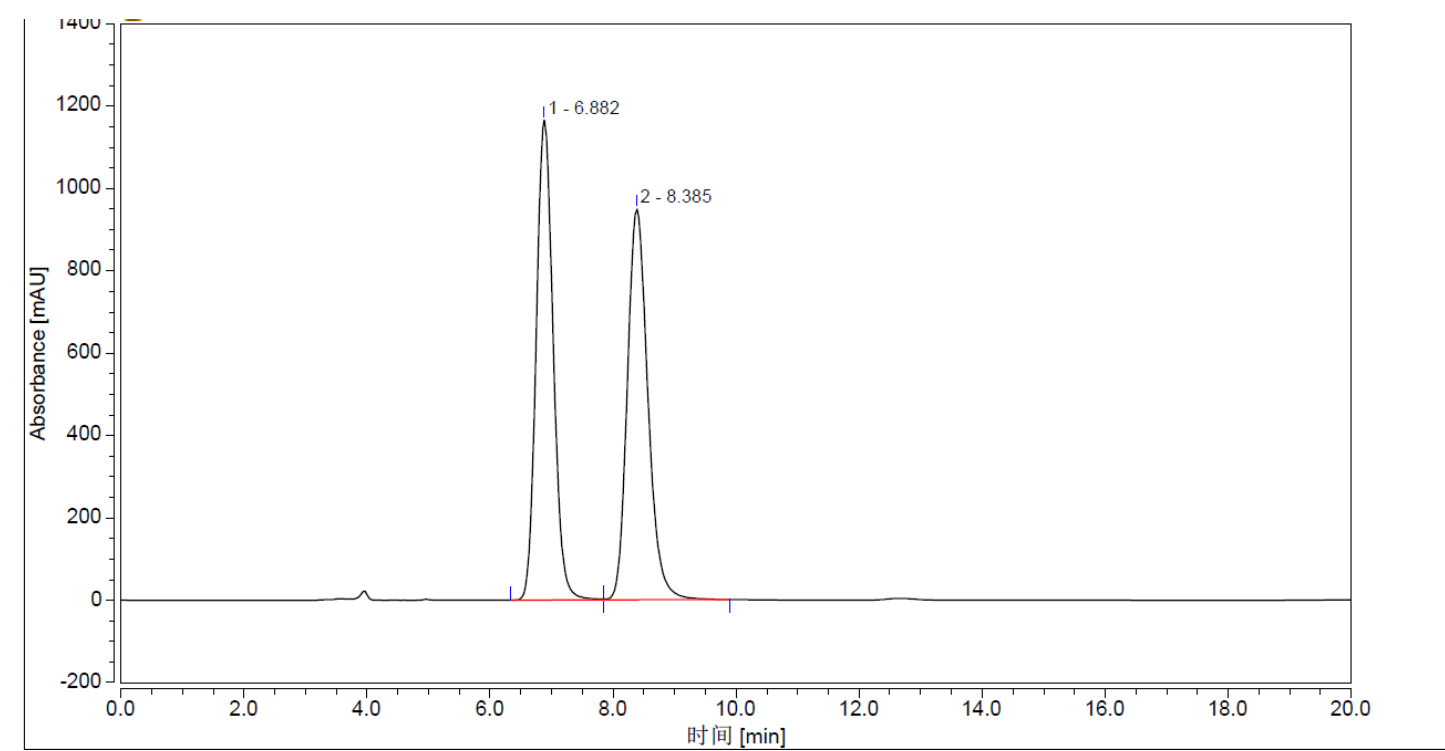

Peak Info

\begin{tabular}{|l|l|l|l|l|}
\hline Peak No & \% Area & Area & RT (min) & Height (mAV) \\
\hline 1 & 50.03 & 371.961 & 6.882 & 1164.989 \\
\hline 2 & 49.97 & 371.229 & 8.385 & 965.136 \\
\hline Total: & 100 & 743.190 & & 2130.125 \\
\hline
\end{tabular}

HBTU: mixture of Fmoc-L-Phg -L-Leu-OtBu (major) and Fmoc-D-Phg -L-Leu-OtBu (minor)

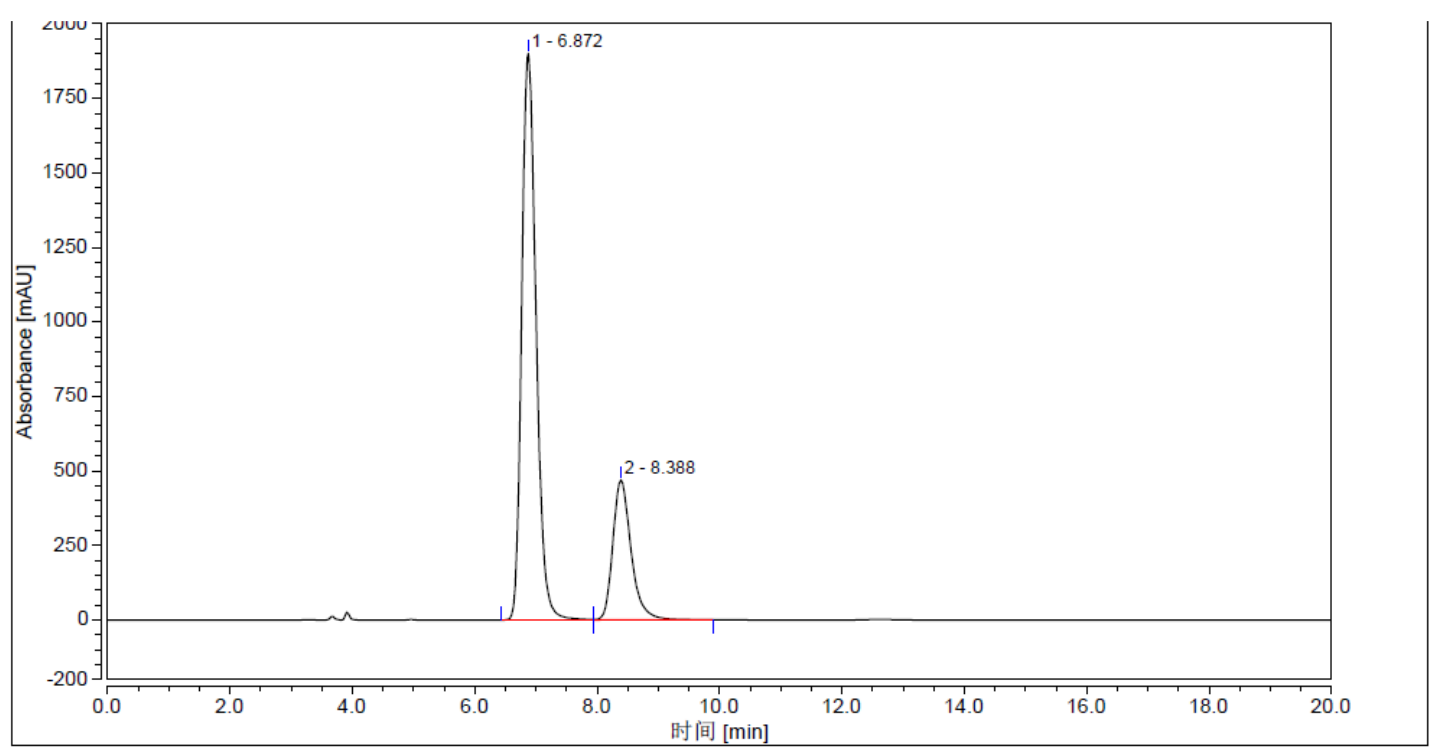

Peak Info

\begin{tabular}{|l|l|l|l|l|}
\hline Peak No & \% Area & Area & RT (min) & Height (mAV) \\
\hline 1 & 76.09 & 515.337 & 6.872 & 1900.035 \\
\hline 2 & 23.91 & 161.952 & 8.388 & 468.739 \\
\hline Total: & 100 & 677.289 & & 2368.774 \\
\hline
\end{tabular}


HATU: mixture of Fmoc-L-Phg -L-Leu-OtBu (major) and Fmoc-D-Phg -L-Leu-OtBu (minor)

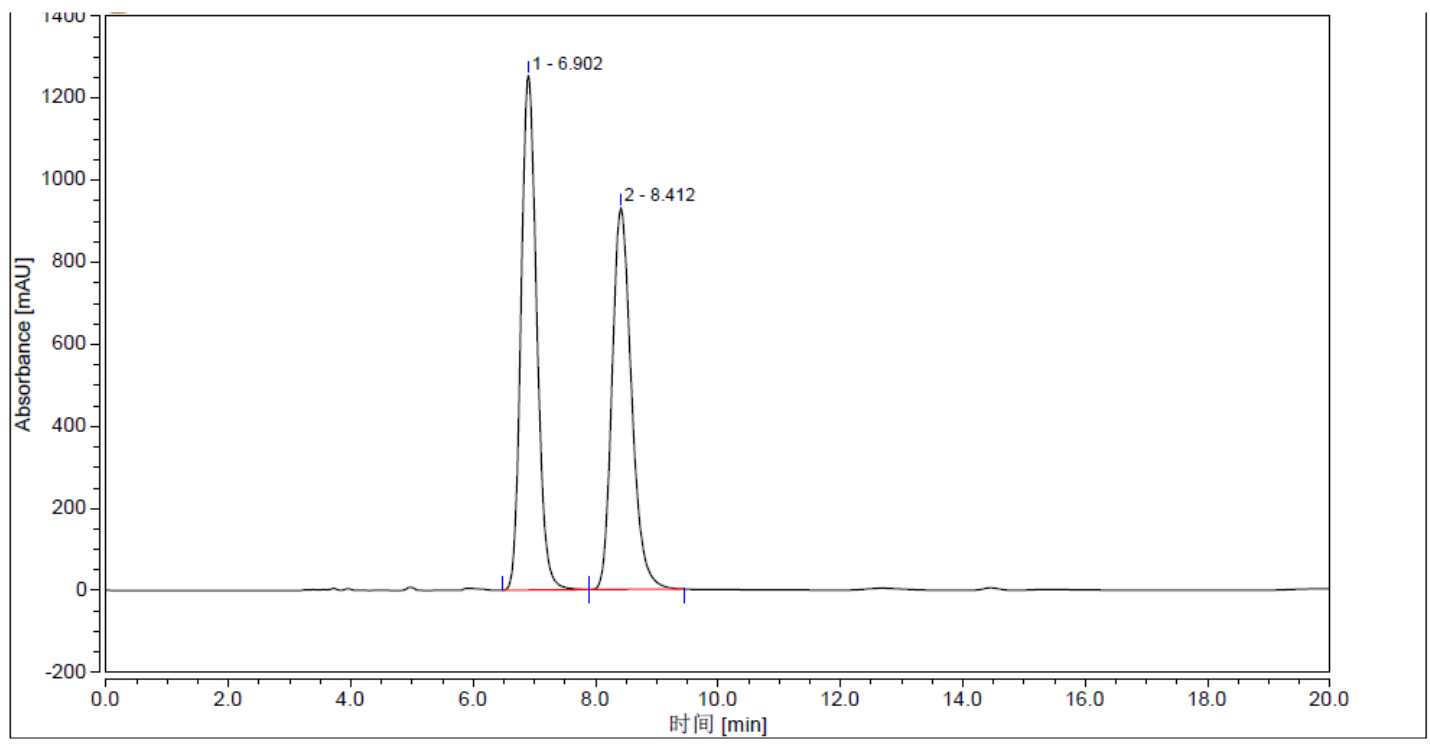

Peak Info

\begin{tabular}{|l|l|l|l|l|}
\hline Peak No & \% Area & Area & RT (min) & Height (mAV) \\
\hline 1 & 52.10 & 372.508 & 6.902 & 1254.977 \\
\hline 2 & 47.90 & 342.492 & 8.412 & 931.162 \\
\hline Total: & 100 & 715.000 & & 2186.139 \\
\hline
\end{tabular}

PyBop: mixture of Fmoc-L-Phg -L-Leu-OtBu (major) and Fmoc-D-Phg -L-Leu-OtBu (minor)

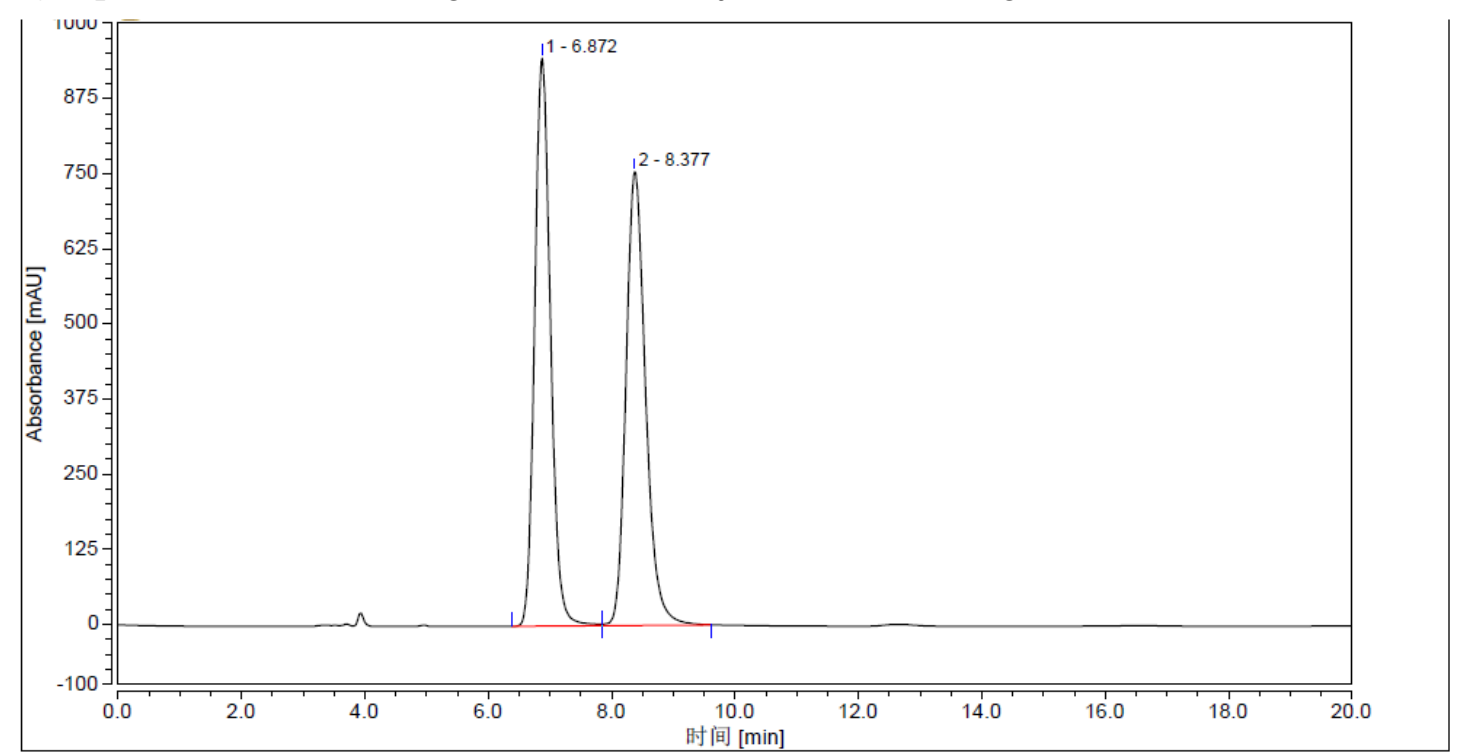

Peak Info

\begin{tabular}{|l|l|l|l|l|}
\hline Peak No & \% Area & Area & RT (min) & Height (mAV) \\
\hline 1 & 50.20 & 280.602 & 6.872 & 944.784 \\
\hline 2 & 49.80 & 278.327 & 8.377 & 754.612 \\
\hline Total: & 100 & 558.929 & & 1699.396 \\
\hline
\end{tabular}


DEPBT: mixture of Fmoc-L-Phg -L-Leu-OtBu (major) and Fmoc-D-Phg -L-Leu-OtBu (minor)

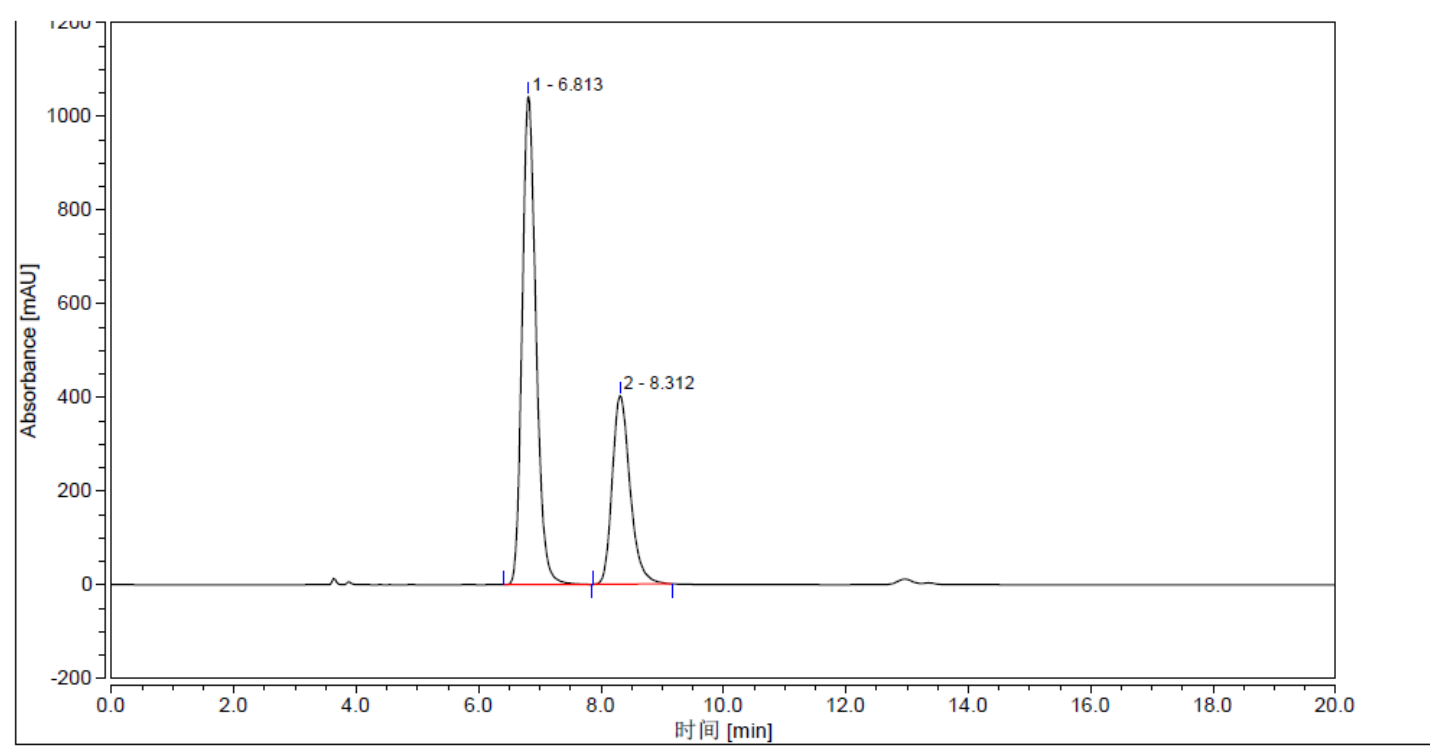

Peak Info

\begin{tabular}{|l|l|l|l|l|}
\hline Peak No & \% Area & Area & RT (min) & Height (mAV) \\
\hline 1 & 66.87 & 274.144 & 6.813 & 1042.994 \\
\hline 2 & 33.13 & 135.843 & 8.312 & 403.260 \\
\hline Total: & 100 & 409.987 & & 1446.254 \\
\hline
\end{tabular}

MYMsA: mixture of Fmoc-L-Phg -L-Leu-OtBu (major) and Fmoc-D-Phg -L-Leu-OtBu (minor)

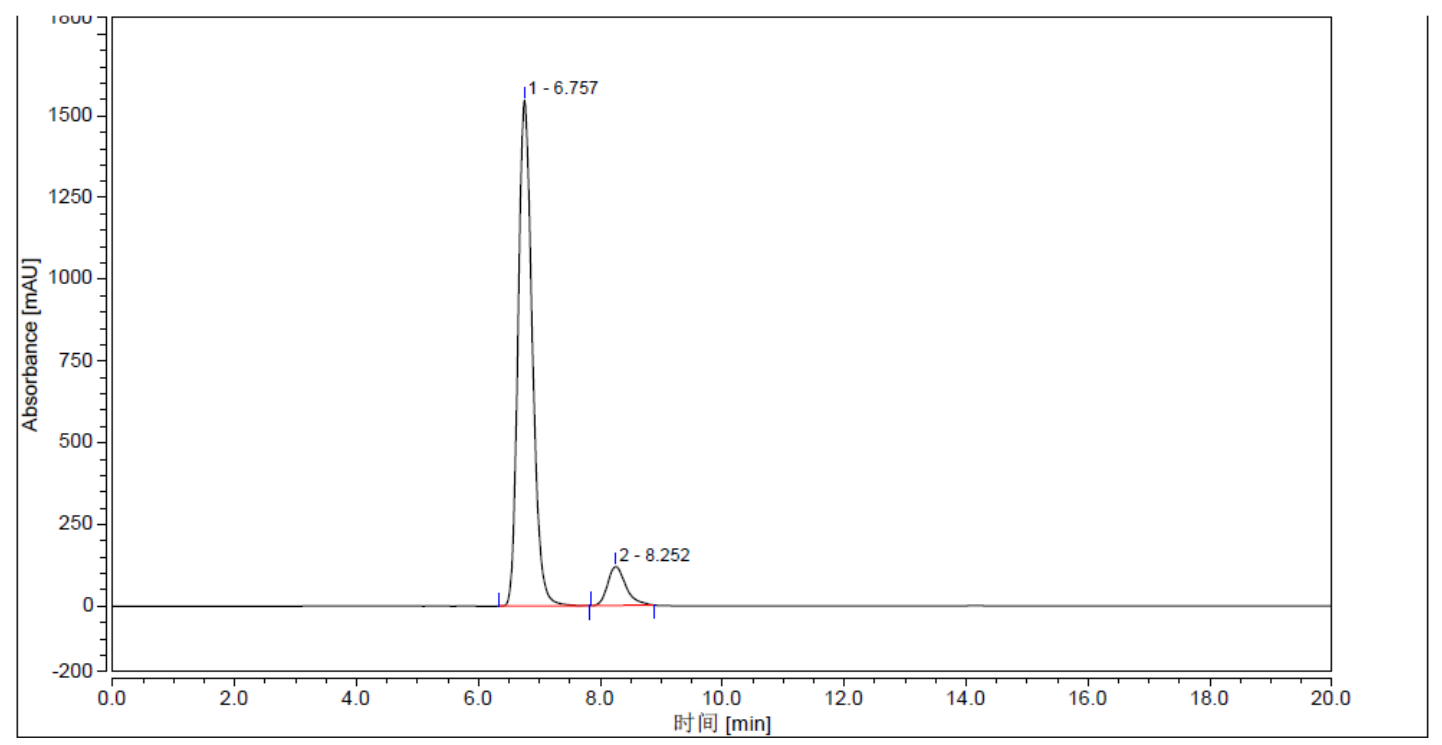

Peak Info

\begin{tabular}{|l|l|l|l|l|}
\hline Peak No & \% Area & Area & RT (min) & Height (mAV) \\
\hline 1 & 91.00 & 418.879 & 6.757 & 1547.796 \\
\hline 2 & 9.00 & 41.408 & 8.252 & 119.093 \\
\hline Total: & 100 & 460.287 & & 1666.889 \\
\hline
\end{tabular}


MYTsA: mixture of Fmoc-L-Phg -L-Leu-OtBu (major) and Fmoc-D-Phg -L-Leu-OtBu (minor)

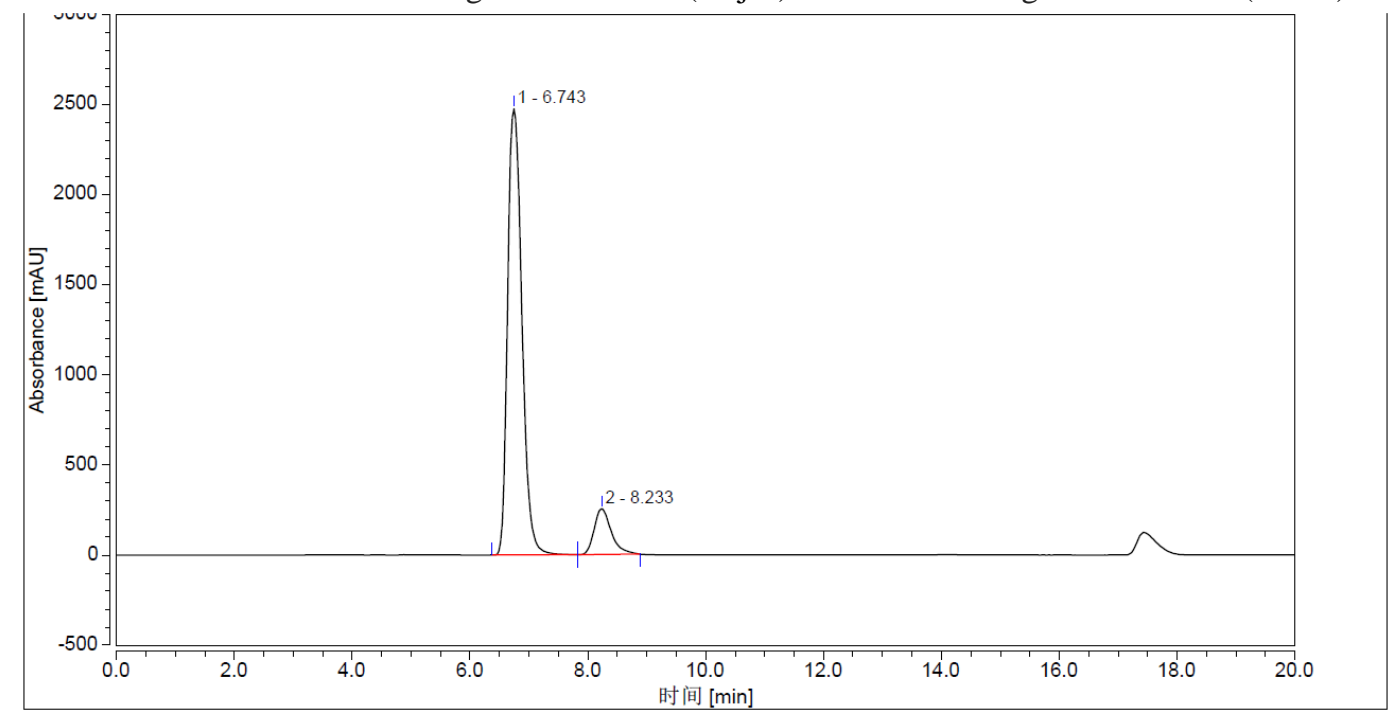

Peak Info

\begin{tabular}{|l|l|l|l|l|}
\hline Peak No & \% Area & Area & RT (min) & Height (mAV) \\
\hline 1 & 89.04 & 694.193 & 6.743 & 2475.698 \\
\hline 2 & 10.96 & 85.426 & 8.233 & 254.093 \\
\hline Total: & 100 & 779.619 & & 2729.791 \\
\hline
\end{tabular}

Study on the racemization of phenylglycine during hydroacyloxylation with MYMsA

Racemate:

1-(N-methylmethylsulfonamido)vinyl 2-((((9H-fluoren-9-yl)methoxy)carbonyl)amino)-2-phenylacetate

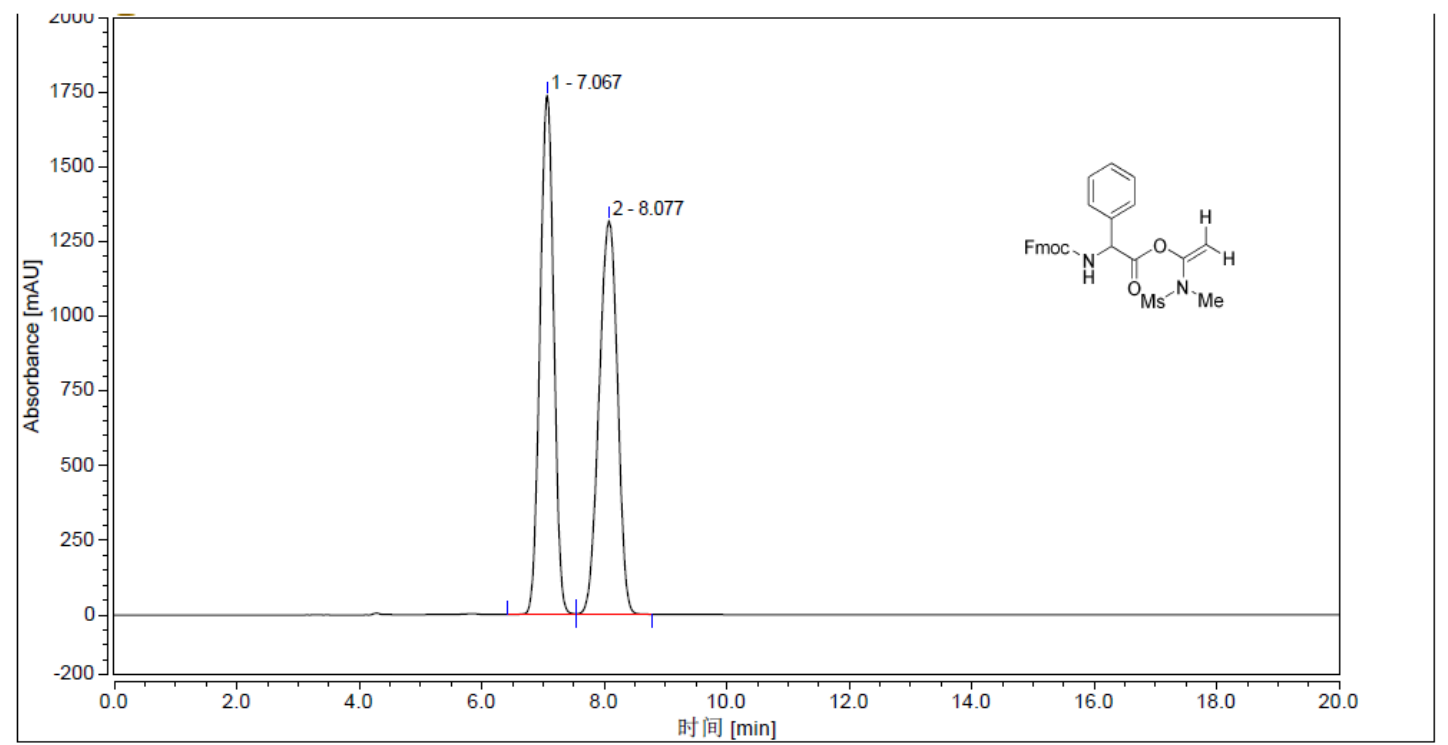

Peak Info

\begin{tabular}{|l|l|l|l|l|}
\hline Peak No & \% Area & Area & RT (min) & Height (mAV) \\
\hline 1 & 49.69 & 454.175 & 7.067 & 1737.262 \\
\hline 2 & 50.31 & 459.886 & 8.077 & 1317.786 \\
\hline Total: & 100 & 914.061 & & 3055.048 \\
\hline
\end{tabular}


1-(N-methylmethylsulfonamido)vinyl $(S)-2-((((9 H$-fluoren-9-yl)methoxy)carbonyl)amino)-2-phenylacetate

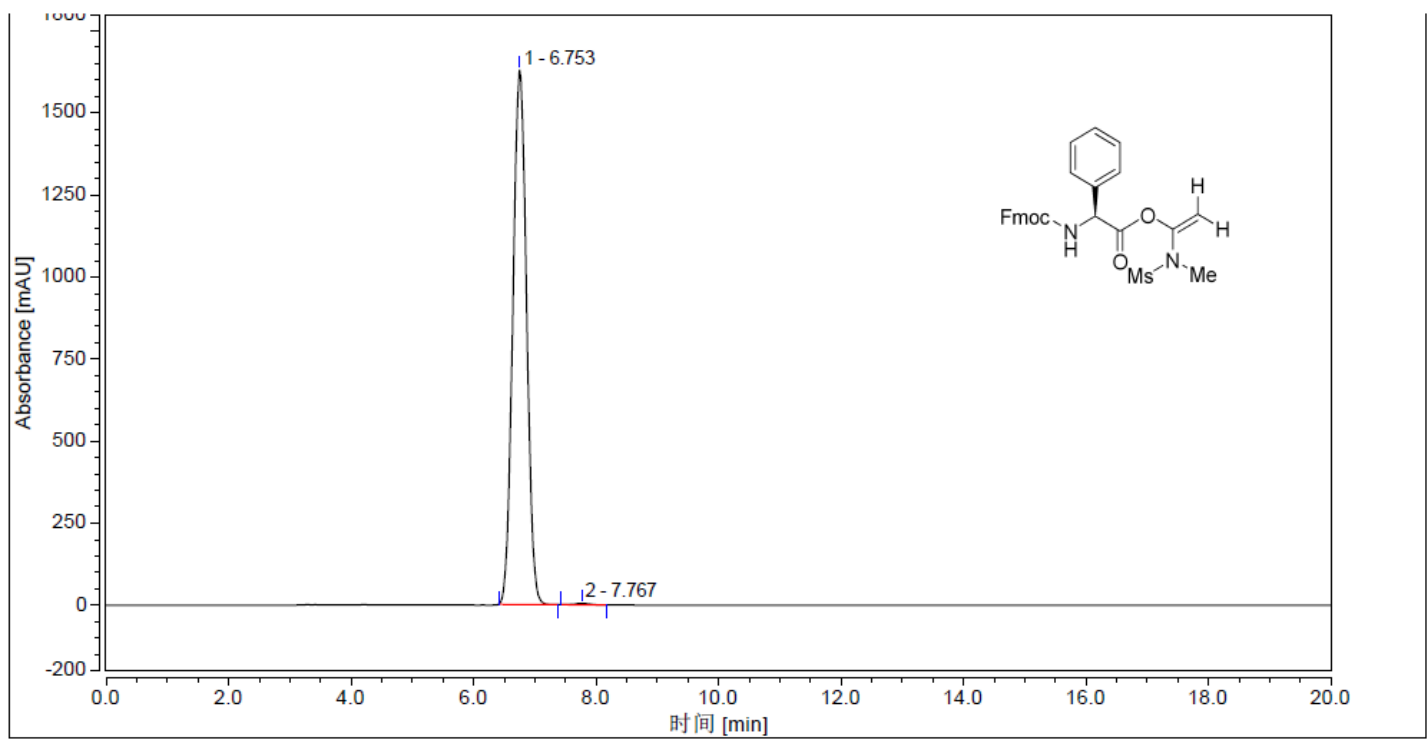

Peak Info

\begin{tabular}{|l|l|l|l|l|}
\hline Peak No & \% Area & Area & RT (min) & Height (mAV) \\
\hline 1 & 99.64 & 422.612 & 6.753 & 1628.853 \\
\hline 2 & 0.36 & 1.539 & 7.767 & 4.949 \\
\hline Total: & 100 & 424.151 & & 1633.802 \\
\hline
\end{tabular}




\section{Ynamide MYTsA Mediated Peptide Bond Formation}<smiles>[R9]NC([R])C(=O)[OH2+]</smiles><smiles>[R]OC(=O)[C@@H]([R])N</smiles><smiles>CN([13CH3])[13CH2]N([13CH3])[13CH3]</smiles>

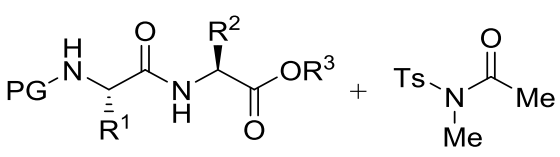

A $5 \mathrm{~mL}$ round-bottomed flask was charged with $\mathrm{N}$-methyl ynetoluenesulfonamide (MYTsA) $(0.2 \mathrm{mmol})$, $\mathrm{CH}_{2} \mathrm{Cl}_{2}(1 \mathrm{~mL})$ and acids $(0.2 \mathrm{mmol})$. The reaction mixture was stirred at room temperature under air until starting material was fully consumed. Amine $(0.24 \mathrm{mmol})$ was added and the reaction mixture was stirred at $35{ }^{\circ} \mathrm{C}$ under air until $\alpha$-acyloxyenamide was fully consumed. The reaction mixture was concentrated and purified by silica gel chromatography to afford the respective peptide.

\section{Larger-scale reactions}

Larger-scale reactions ( $10 \& 20 \mathrm{mmol}$ scale) to provide 5 or 11 gram dipeptide were carried out with Fmoc-L-Ser(OtBu)-L-Leu-(OtBu) as the model substrate. As shown below, excellent efficiency were retained for $10 \& 20 \mathrm{mmol}$ reactions.

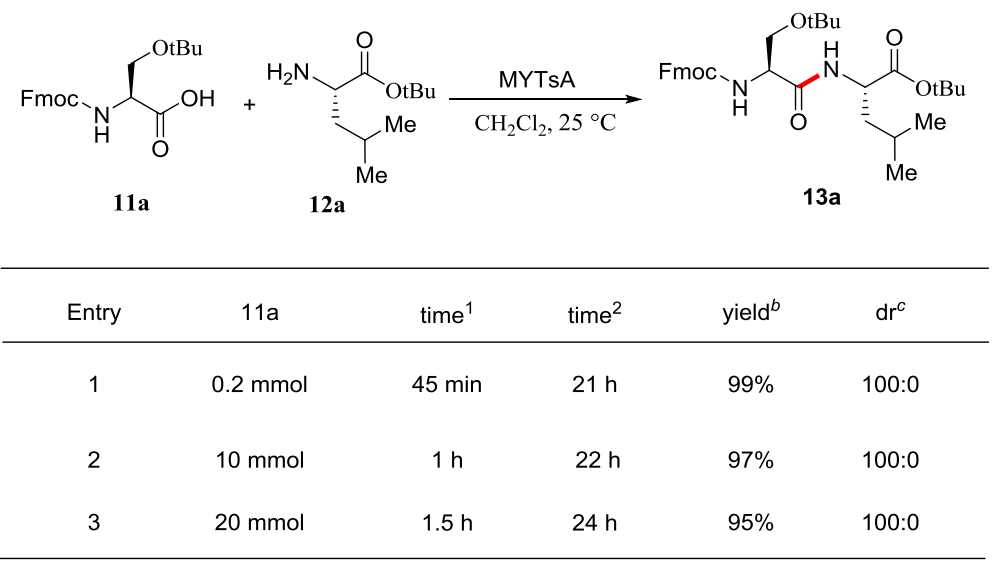

${ }^{a}$ Reaction conditions: 11 a ( 1 equv), $12 \mathrm{a}$ ( 1.1 equiv), MYTsA (1.1 equiv), ${ }^{b}$ Isolated yield. ${ }^{c}$ Determined by HPLC analysis. 


\section{Copies of HPLC Chromatograms}

1:1 mixture of Fmoc-L-Ser(tBu)-L-Leu-OtBu and Fmoc-D-Ser(tBu)-L-Leu-OtBu

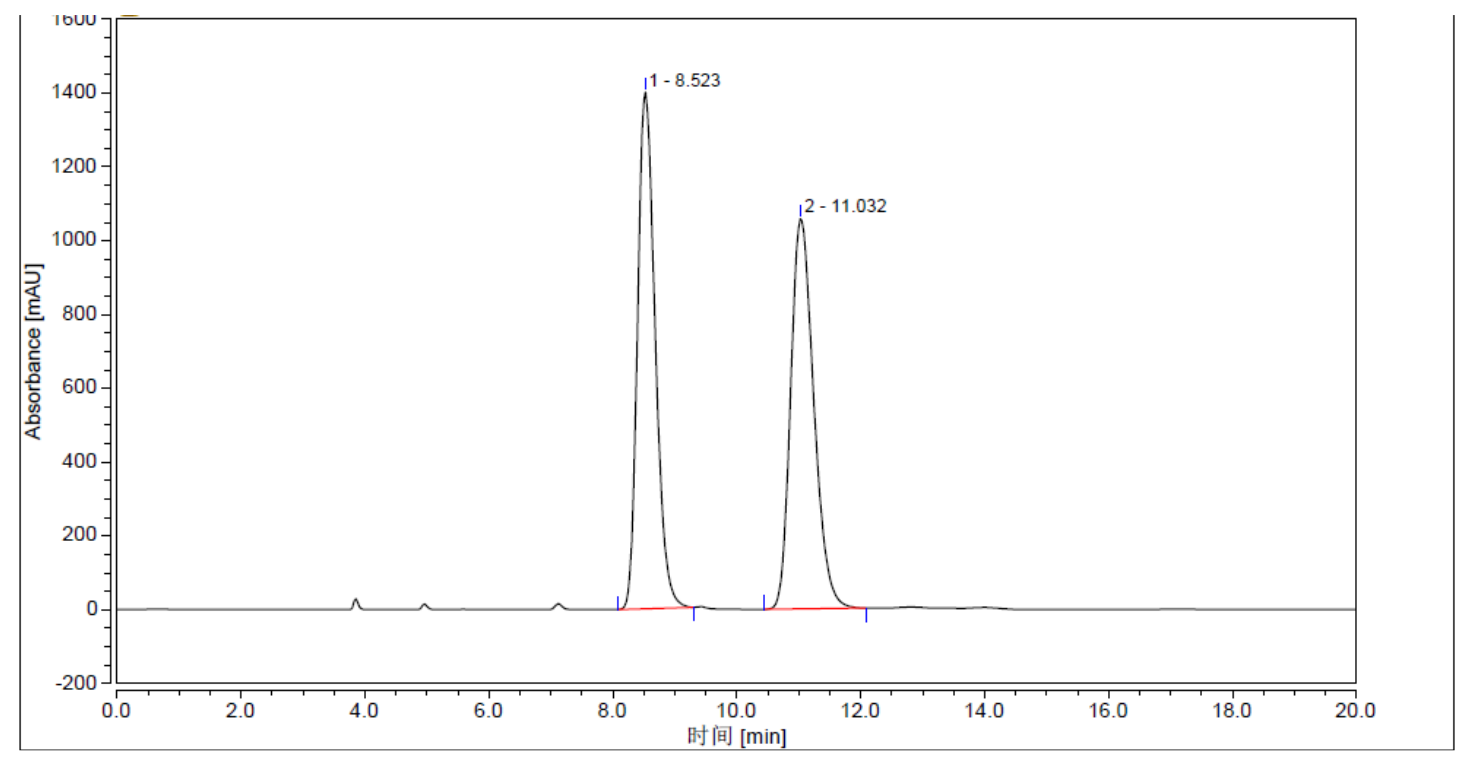

Peak Info

\begin{tabular}{|l|l|l|l|l|}
\hline Peak No & \% Area & Area & RT $(\mathrm{min})$ & Height $(\mathrm{mAV})$ \\
\hline 1 & 50.22 & 460.450 & 8.523 & 1400.183 \\
\hline 2 & 49.78 & 456.369 & 11.032 & 1058.451 \\
\hline Total: & 100 & 916.818 & & 2458.635 \\
\hline
\end{tabular}

MYTsA: mixture of Fmoc-D-Ser(tBu)-L-Leu-OtBu (minor) and Fmoc-L-Ser(tBu)-L-Leu-

$\mathrm{OtBu}$ (major)) (0.2 mmol scale)

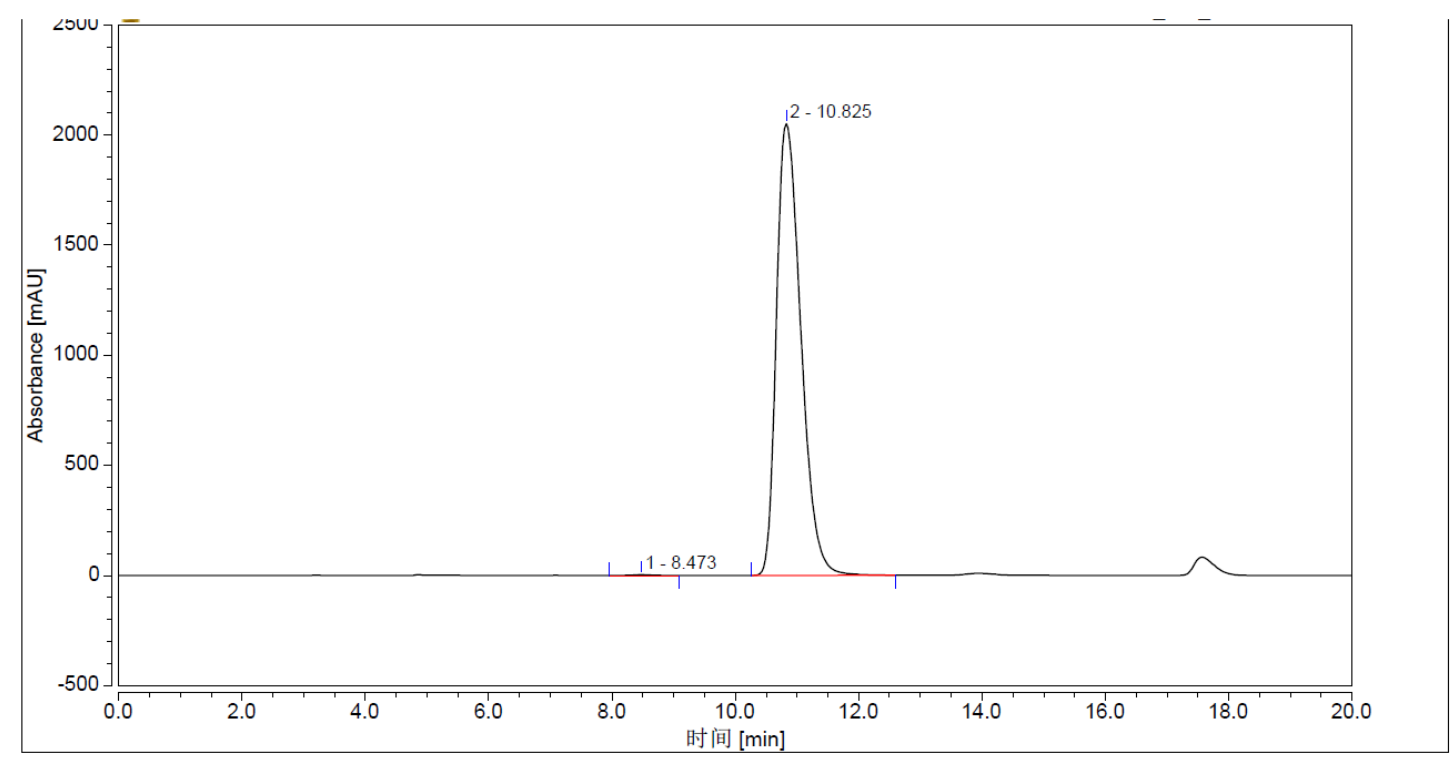

Peak Info

\begin{tabular}{|l|l|l|l|l|}
\hline Peak No & \% Area & Area & RT $(\min )$ & Height $(\mathrm{mAV})$ \\
\hline 1 & 0.13 & 1.217 & 8.473 & 3.666 \\
\hline 2 & 99.87 & 940.820 & 10.825 & 2051.845 \\
\hline Total: & 100 & 942.037 & & 2055.511 \\
\hline
\end{tabular}


MYTsA: mixture of Fmoc-D-Ser(tBu)-L-Leu-OtBu (minor) and Fmoc-L-Ser(tBu)-L-LeuOtBu(major)) (10 mmol scale)

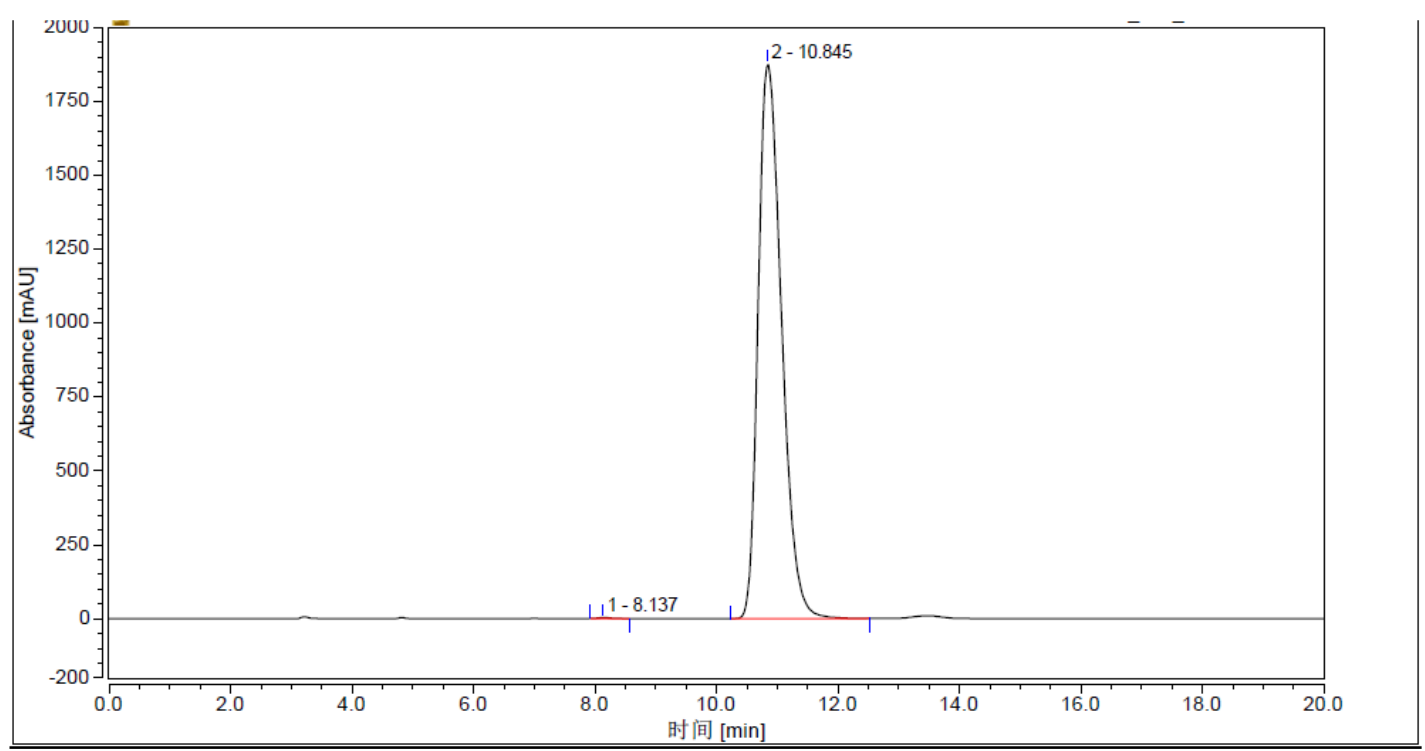

Peak Info

\begin{tabular}{|l|l|l|l|l|}
\hline Peak No & \% Area & Area & RT (min) & Height (mAV) \\
\hline 1 & 0.12 & 0.993 & 8.137 & 3.666 \\
\hline 2 & 99.88 & 837.502 & 10.845 & 1876.068 \\
\hline Total: & 100 & 942.037 & & 1879.734 \\
\hline
\end{tabular}

MYTsA: mixture of Fmoc-D-Ser(tBu)-L-Leu-OtBu (minor) and Fmoc-L-Ser(tBu)-L-LeuOtBu(major)) (20 mmol scale)

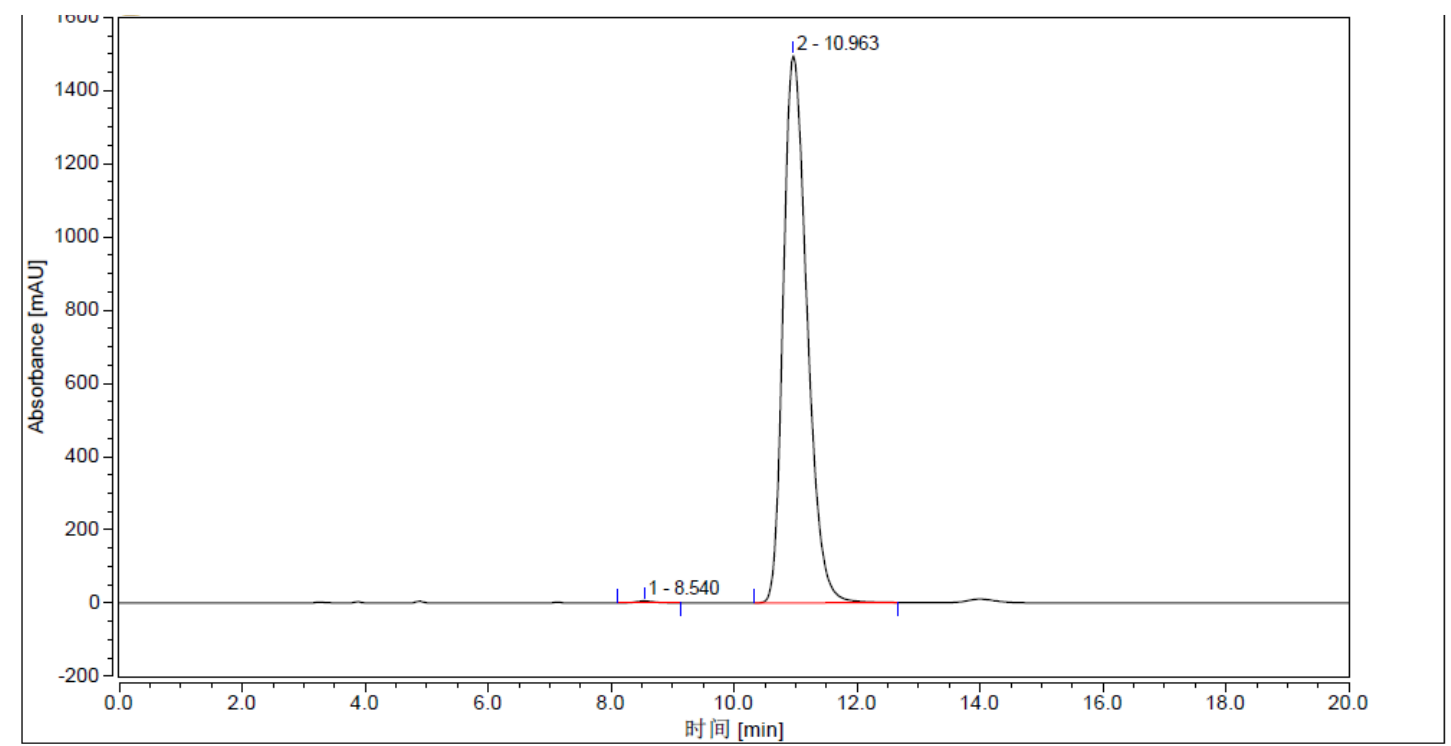

Peak Info

\begin{tabular}{|l|l|l|l|l|}
\hline Peak No & \% Area & Area & RT (min) & Height (mAV) \\
\hline 1 & 0.26 & 1.751 & 8.540 & 5.137 \\
\hline 2 & 99.74 & 669.325 & 10.963 & 1495.009 \\
\hline Total: & 100 & 671.076 & & 1500.146 \\
\hline
\end{tabular}




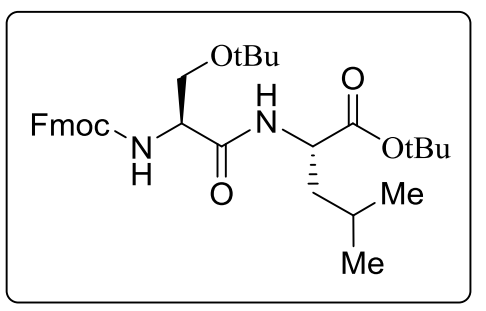

White solid, 99\% yield (109.3 mg). ${ }^{1} \mathrm{H}$ NMR $\left(400 \mathrm{MHz}, \mathrm{CDCl}_{3}\right) \delta$ $7.75(\mathrm{~d}, J=7.5 \mathrm{~Hz}, 2 \mathrm{H}), 7.65-7.55(\mathrm{~m}, 2 \mathrm{H}), 7.39$ (t, $J=7.5 \mathrm{~Hz}$, $2 \mathrm{H}), 7.31(\mathrm{t}, J=7.4 \mathrm{~Hz}, 2 \mathrm{H}), 7.25(\mathrm{~d}, J=8.8 \mathrm{~Hz}, 1 \mathrm{H}), 5.81(\mathrm{~d}, 1 \mathrm{H})$, $4.50(\mathrm{~d}, J=4.9 \mathrm{~Hz}, 1 \mathrm{H}), 4.45-4.33(\mathrm{~m}, 2 \mathrm{H}), 4.27-4.21(\mathrm{~m}, 2 \mathrm{H})$, $3.84(\mathrm{dd}, J=8.4,3.7 \mathrm{~Hz}, 1 \mathrm{H}), 3.41(\mathrm{t}, J=8.2 \mathrm{~Hz}, 1 \mathrm{H}), 1.75-1.60$ $(\mathrm{m}, 2 \mathrm{H}), 1.58-1.50(\mathrm{~m}, 1 \mathrm{H}), 1.46(\mathrm{~s}, 9 \mathrm{H}), 1.23(\mathrm{~s}, 9 \mathrm{H}), 0.96(\mathrm{~d}, J$ $=6.3 \mathrm{~Hz}, 6 \mathrm{H}) ;{ }^{13} \mathrm{C} \mathrm{NMR}\left(100 \mathrm{MHz}, \mathrm{CDCl}_{3}\right) \delta 171.5,169.8,155.9,143.7,141.2,127.6,127.0,125.0$, 119.9, 81.6, 74.1, 67.0, 61.7, 54.2, 51.6, 47.1, 41.8, 27.9, 27.3, 24.8, 22.7, 22.1 ppm; HRMS m/z (ESI) calcd for $\mathrm{C}_{32} \mathrm{H}_{44} \mathrm{~N}_{2} \mathrm{NaO}_{6}(\mathrm{M}+\mathrm{Na})^{+}$: 575.3092 , found 575.3090 .

Fmoc-L-Asp-L-Tyr(tBu)-OtBu (13b)

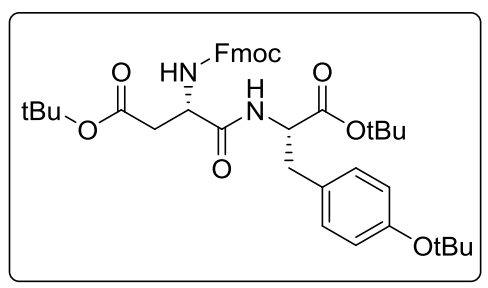

White solid, 97\% yield (131.7 mg). ${ }^{1} \mathrm{H}$ NMR $\left(400 \mathrm{MHz}, \mathrm{CDCl}_{3}\right) \delta$ $7.76(\mathrm{~d}, J=7.5 \mathrm{~Hz}, 2 \mathrm{H}), 7.59(\mathrm{~d}, J=7.3 \mathrm{~Hz}, 2 \mathrm{H}), 7.39(\mathrm{t}, J=7.4$ $\mathrm{Hz}, 2 \mathrm{H}), 7.31(\mathrm{t}, J=7.2 \mathrm{~Hz}, 2 \mathrm{H}), 7.07(\mathrm{t}, J=8.3 \mathrm{~Hz}, 3 \mathrm{H}), 6.89(\mathrm{~d}$, $J=8.3 \mathrm{~Hz}, 2 \mathrm{H}), 5.99(\mathrm{~d}, J=8.1 \mathrm{~Hz}, 1 \mathrm{H}), 4.66(\mathrm{dd}, J=13.4,6.3 \mathrm{~Hz}$, $1 \mathrm{H}), 4.58-4.53(\mathrm{~m}, 1 \mathrm{H}), 4.38(\mathrm{p}, J=10.4 \mathrm{~Hz}, 2 \mathrm{H}), 4.23(\mathrm{t}, J=7.1$ $\mathrm{Hz}, 1 \mathrm{H}), 3.10-2.96(\mathrm{~m}, 2 \mathrm{H}), 2.90(\mathrm{dd}, J=17.0,3.9 \mathrm{~Hz}, 1 \mathrm{H}), 2.63$ (dd, $J=16.7,5.9 \mathrm{~Hz}, 1 \mathrm{H}), 1.45$ (s, 9H), $1.36(\mathrm{~s}, 9 \mathrm{H}), 1.29(\mathrm{~s}, 9 \mathrm{H}) ;{ }^{13} \mathrm{C} \mathrm{NMR}\left(100 \mathrm{MHz}, \mathrm{CDCl}_{3}\right) \delta 171.0$, 169.9, 169.9, 155.9, 154.3, 143.6, 141.2, 130.9, 129.9, 127.7, 127.0, 125.0, 124.0, 119.9, 82.2, 81.8, 78.2, 67.3, 54.0, 51.0, 47.0, 37.4, 37.4, 28.7, 28.0, $27.8 \mathrm{ppm}$; HRMS m/z (ESI) calcd for $\mathrm{C}_{40} \mathrm{H}_{50} \mathrm{~N}_{2} \mathrm{NaO}_{8}$ $(\mathrm{M}+\mathrm{Na})^{+}:$709.3459, found 709.3493 .

\section{Fmoc-L-Trp(Boc)-L-Tyr(tBu)-OtBu (13c)}

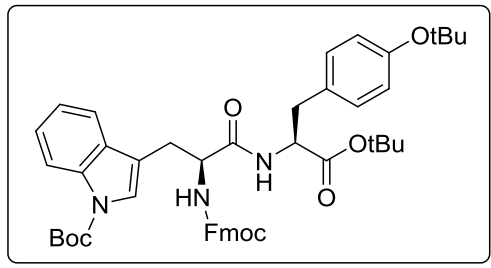

Glassy solid, $96 \%$ yield $(153.8 \mathrm{mg}) .{ }^{1} \mathrm{H}$ NMR $\left(400 \mathrm{MHz}, \mathrm{CDCl}_{3}\right)$ $\delta 8.14(\mathrm{~d}, J=7.3 \mathrm{~Hz}, 1 \mathrm{H}), 7.75(\mathrm{~d}, J=7.5 \mathrm{~Hz}, 2 \mathrm{H}), 7.62(\mathrm{~d}, J=7.0$ $\mathrm{Hz}, 1 \mathrm{H}), 7.57-7.49(\mathrm{~m}, 3 \mathrm{H}), 7.42-7.27(\mathrm{~m}, 5 \mathrm{H}), 7.25-7.21(\mathrm{~m}$, $1 \mathrm{H}), 6.85(\mathrm{~d}, J=7.3 \mathrm{~Hz}, 2 \mathrm{H}), 6.77(\mathrm{~d}, J=8.3 \mathrm{~Hz}, 2 \mathrm{H}), 6.36(\mathrm{~d}, J$ $=6.2 \mathrm{~Hz}, 1 \mathrm{H}), 5.51(\mathrm{~d}, J=4.9 \mathrm{~Hz}, 1 \mathrm{H}), 4.55(\mathrm{dd}, J=12.8,6.0 \mathrm{~Hz}$, 2H), $4.46-4.37(\mathrm{~m}, 1 \mathrm{H}), 4.37-4.29(\mathrm{~m}, 1 \mathrm{H}), 4.20(\mathrm{t}, J=6.7 \mathrm{~Hz}, 1 \mathrm{H}), 3.34-3.21(\mathrm{~m}, 1 \mathrm{H}), 3.14(\mathrm{dd}, J$ $=14.2,7.0 \mathrm{~Hz}, 1 \mathrm{H}), 2.94(\mathrm{~d}, J=5.4 \mathrm{~Hz}, 2 \mathrm{H}), 1.60(\mathrm{~s}, 9 \mathrm{H}), 1.30(\mathrm{~s}, 9 \mathrm{H}), 1.26(\mathrm{~s}, 9 \mathrm{H}) ;{ }^{13} \mathrm{C}$ NMR $(100$ $\left.\mathrm{MHz}, \mathrm{CDCl}_{3}\right) \delta 170.1,169.7,155.9,154.3,149.4,143.7,143.7,141.2,135.5,130.7,130.2,129.8,127.7$, 127.1, 125.1, 124.7, 124.6, 123.9, 122.8, 119.9, 118.9, 115.4, 115.1, 83.6, 82.3, 78.3, 67.3, 55.0, 53.9, 47.0, 37.3, 28.8, 28.2, 28.1, 27.8 ppm; HRMS m/z (ESI) calcd for $\mathrm{C}_{48} \mathrm{H}_{55} \mathrm{~N}_{3} \mathrm{NaO}_{8}(\mathrm{M}+\mathrm{Na})^{+}:$824.3881, found 802.3906 .

\section{Fmoc-L-Ser-L-Tyr(tBu)-OtBu (13d)}

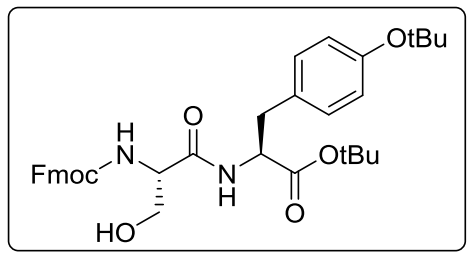

Glassy solid, 98\% yield (118.0 mg). ${ }^{1} \mathrm{H}$ NMR $\left(400 \mathrm{MHz}, \mathrm{CDCl}_{3}\right) \delta$ $7.76(\mathrm{~d}, J=7.5 \mathrm{~Hz}, 2 \mathrm{H}), 7.60(\mathrm{~d}, J=7.3 \mathrm{~Hz}, 2 \mathrm{H}), 7.39(\mathrm{t}, J=7.4 \mathrm{~Hz}$, $2 \mathrm{H}), 7.34-7.28(\mathrm{~m}, 2 \mathrm{H}), 7.04(\mathrm{~d}, J=8.4 \mathrm{~Hz}, 2 \mathrm{H}), 6.95(\mathrm{~s}, 1 \mathrm{H}), 6.88$ $(\mathrm{d}, J=8.4 \mathrm{~Hz}, 2 \mathrm{H}), 5.84(\mathrm{~s}, 1 \mathrm{H}), 4.70(\mathrm{dd}, J=14.1,6.4 \mathrm{~Hz}, 1 \mathrm{H})$, $4.47-4.33(\mathrm{~m}, 2 \mathrm{H}), 4.22(\mathrm{t}, J=7.0 \mathrm{~Hz}, 2 \mathrm{H}), 4.01(\mathrm{~d}, J=10.1 \mathrm{~Hz}$, $1 \mathrm{H}), 3.64(\mathrm{~d}, J=5.0 \mathrm{~Hz}, 1 \mathrm{H}), 3.29(\mathrm{~s}, 1 \mathrm{H}), 3.03(\mathrm{qd}, J=14.1,6.4 \mathrm{~Hz}, 2 \mathrm{H}), 1.39(\mathrm{~s}, 9 \mathrm{H}), 1.29(\mathrm{~s}, 9 \mathrm{H})$; ${ }^{13} \mathrm{C}$ NMR $\left(100 \mathrm{MHz}, \mathrm{CDCl}_{3}\right) \delta 170.6,170.4,156.4,154.4,143.7,143.6,141.3,130.7,129.8,127.7$, 
127.1, 125.1, 124.1, 120.0, 82.7, 78.4, 67.3, 62.9, 55.3, 54.0, 47.0, 37.1, 28.8, 27.9 ppm; HRMS m/z (ESI) calcd for $\mathrm{C}_{35} \mathrm{H}_{42} \mathrm{~N}_{2} \mathrm{NaO}_{7}(\mathrm{M}+\mathrm{Na})^{+}:$625.2884, found 625.2880.

\section{Cbz-L-Ser-L-Tyr(tBu)-OtBu (13e)}

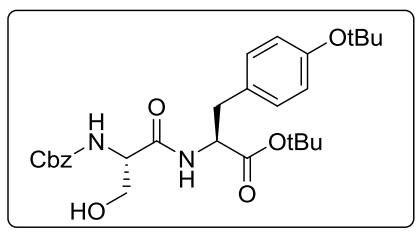

Glassy solid, $98 \%$ yield (100.7 mg). ${ }^{1} \mathrm{H}$ NMR (400 MHz, $\left.\mathrm{CDCl}_{3}\right) \delta 7.43$ $-7.29(\mathrm{~m}, 5 \mathrm{H}), 7.04(\mathrm{~d}, J=8.4 \mathrm{~Hz}, 2 \mathrm{H}), 6.96(\mathrm{~d}, J=7.3 \mathrm{~Hz}, 1 \mathrm{H}), 6.92$ $-6.85(\mathrm{~m}, 2 \mathrm{H}), 5.79(\mathrm{~d}, J=7.3 \mathrm{~Hz}, 1 \mathrm{H}), 5.16-5.03(\mathrm{~m}, 2 \mathrm{H}), 4.69$ (dd, $J=14.2,6.4 \mathrm{~Hz}, 1 \mathrm{H}), 4.23(\mathrm{~s}, 1 \mathrm{H}), 4.08-3.92(\mathrm{~m}, 1 \mathrm{H}), 3.61(\mathrm{dt}, J=$ $11.5,6.9 \mathrm{~Hz}, 1 \mathrm{H}), 3.24(\mathrm{~s}, 1 \mathrm{H}), 3.02(\mathrm{qd}, J=14.0,6.4 \mathrm{~Hz}, 2 \mathrm{H}), 1.39$ (s, 9H), $1.31(\mathrm{~s}, 9 \mathrm{H}) ;{ }^{13} \mathrm{C} \mathrm{NMR}\left(100 \mathrm{MHz}, \mathrm{CDCl}_{3}\right) \delta 170.6,170.5,156.4,154.4,136.1,130.8,129.9,128.6$, 128.3, 128.1, 124.2, 82.7, 78.5, 67.3, 62.9, 55.4, 54.0, 37.2, 28.8, 28.0 ppm; HRMS m/z (ESI) calcd for $\mathrm{C}_{28} \mathrm{H}_{38} \mathrm{~N}_{2} \mathrm{NaO}_{7}(\mathrm{M}+\mathrm{Na})^{+}:$537.2571, found 537.2572.

\section{Boc-L-Thr-L-Tyr(tBu)-OtBu (13f)}

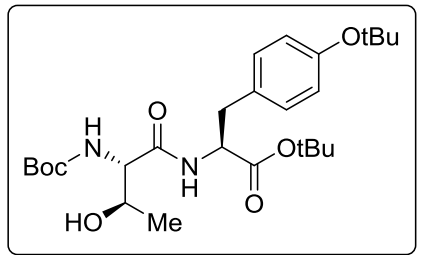

Glassy solid, 96\% yield (94.8 mg). ${ }^{1} \mathrm{H} \mathrm{NMR}\left(400 \mathrm{MHz}, \mathrm{CDCl}_{3}\right) \delta 7.05$ $(\mathrm{d}, J=8.4 \mathrm{~Hz}, 3 \mathrm{H}), 6.88(\mathrm{~d}, J=8.4 \mathrm{~Hz}, 2 \mathrm{H}), 5.45(\mathrm{~d}, J=7.5 \mathrm{~Hz}, 1 \mathrm{H})$, $4.67(\mathrm{dd}, J=13.7,6.5 \mathrm{~Hz}, 1 \mathrm{H}), 4.28(\mathrm{~d}, J=4.7 \mathrm{~Hz}, 1 \mathrm{H}), 4.07$ (d, $J=6.9$ $\mathrm{Hz}, 1 \mathrm{H}), 2.99$ (t, $J=6.1 \mathrm{~Hz}, 2 \mathrm{H}), 1.43(\mathrm{~s}, 9 \mathrm{H}), 1.36(\mathrm{~s}, 9 \mathrm{H}), 1.30(\mathrm{~s}, 9 \mathrm{H})$, $1.13(\mathrm{~d}, J=6.4 \mathrm{~Hz}, 3 \mathrm{H}) ;{ }^{13} \mathrm{C} \mathrm{NMR}\left(100 \mathrm{MHz} \mathrm{CDCl}_{3}\right) \delta 170.8,170.3$, 156.2, 154.3, 130.9, 129.8, 124.0, 82.3, 80.2, 78.3, 66.9, 58.3, 53.8, 37.5, 28.8, 28.2, 27.9, 18.2 ppm; HRMS m/z (ESI) calcd for $\mathrm{C}_{26} \mathrm{H}_{42} \mathrm{~N}_{2} \mathrm{NaO}_{7}(\mathrm{M}+\mathrm{Na})^{+}:$517.2884, found 517.2889.

Fmoc-L-Thr-L-Thr-OtBu (13g)

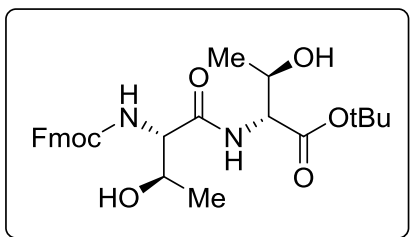

Glassy solid, 97\% yield (96.6 mg). ${ }^{1} \mathrm{H}$ NMR (400 $\left.\mathrm{MHz}, \mathrm{CDCl}_{3}\right) \delta 7.73$ $(\mathrm{d}, J=7.5 \mathrm{~Hz}, 2 \mathrm{H}), 7.57(\mathrm{~d}, J=6.6 \mathrm{~Hz}, 2 \mathrm{H}), 7.36(\mathrm{t}, J=7.5 \mathrm{~Hz}, 3 \mathrm{H})$, $7.27(\mathrm{t}, J=7.5 \mathrm{~Hz}, 2 \mathrm{H}), 6.15(\mathrm{~s}, 1 \mathrm{H}), 4.49-4.38(\mathrm{~m}, 2 \mathrm{H}), 4.38-4.22$ $(\mathrm{m}, 4 \mathrm{H}), 4.19(\mathrm{t}, J=7.0 \mathrm{~Hz}, 1 \mathrm{H}), 1.45(\mathrm{~s}, 9 \mathrm{H}), 1.19(\mathrm{~d}, J=6.4 \mathrm{~Hz}, 6 \mathrm{H})$; ${ }^{13} \mathrm{C} \mathrm{NMR}\left(100 \mathrm{MHz}, \mathrm{CDCl}_{3}\right) \delta 171.2,169.9,156.7,143.6,141.2,127.7$, 127.0, 125.0, 119.9, 82.7, 68.1, 67.4, 67.3, 59.0, 58.5, 47.0, 27.9, 20.1, 18.0 ppm; HRMS m/z (ESI) calcd for $\mathrm{C}_{27} \mathrm{H}_{34} \mathrm{~N}_{2} \mathrm{NaO}_{7}(\mathrm{M}+\mathrm{Na})^{+}:$521.2258, found 521.2250.

\section{Cbz-L-Thr-L-Thr-OtBu (13h)}

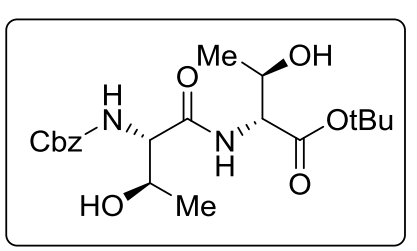

Glassy solid, $98 \%$ yield (80.4 mg). ${ }^{1} \mathrm{H}$ NMR (400 MHz, $\left.\mathrm{CDCl}_{3}\right) \delta 7.40$ -7.29 (m, 5H), 6.09 (s, 1H), 5.09 (s, 2H), $4.43(\mathrm{dd}, J=8.9,3.2 \mathrm{~Hz}, 1 \mathrm{H})$, $4.33-4.18(\mathrm{~m}, 3 \mathrm{H}), 3.99(\mathrm{~s}, 1 \mathrm{H}), 3.50(\mathrm{~s}, 1 \mathrm{H}), 2.59$ (s, 1H), $1.45(\mathrm{~s}, 9 \mathrm{H})$, $1.22-1.11(\mathrm{~m}, 6 \mathrm{H}) ;{ }^{13} \mathrm{C}$ NMR $\left(100 \mathrm{MHz}, \mathrm{CDCl}_{3}\right) \delta 171.2,169.9,156.7$, $136.1,128.5,128.1,127.9,82.7,68.1,67.3,67.2,59.1,58.4,27.9,20.0$, 18.1ppm; HRMS m/z (ESI) calcd for $\mathrm{C}_{20} \mathrm{H}_{30} \mathrm{~N}_{2} \mathrm{NaO}_{7}(\mathrm{M}+\mathrm{Na})^{+}: 433.1945$, found 433.1932.

\section{Boc-L-Thr-L-Thr-OtBu (13i)}<smiles>CCCOC(=O)[C@@H](NC(=O)[C@@H](NC(=O)OCC)C(C)O)[C@@H](C)O</smiles>

Glassy solid, 97\% yield (72.2 mg). ${ }^{1} \mathrm{H}$ NMR (400 MHz, $\left.\mathrm{CDCl}_{3}\right) \delta 7.35$ $(\mathrm{d}, J=7.7 \mathrm{~Hz}, 1 \mathrm{H}), 5.73(\mathrm{~s}, 1 \mathrm{H}), 4.42(\mathrm{dd}, J=8.7,2.4 \mathrm{~Hz}, 1 \mathrm{H}), 4.35-$ $4.09(\mathrm{~m}, 3 \mathrm{H}), 1.43(\mathrm{~d}, J=10.9 \mathrm{~Hz}, 18 \mathrm{H}), 1.18(\mathrm{t}, J=6.2 \mathrm{~Hz}, 6 \mathrm{H}) ;{ }^{13} \mathrm{C}$ NMR $\left(100 \mathrm{MHz}, \mathrm{CDCl}_{3}\right) \delta 171.7,169.8,156.2,82.6,80.3,68.2,67.3$, 58.7, 58.4, 28.2, 27.9, 20.0, $18.2 \mathrm{ppm}$; HRMS m/z (ESI) calcd for $\mathrm{C}_{17} \mathrm{H}_{32} \mathrm{~N}_{2} \mathrm{NaO}_{7}(\mathrm{M}+\mathrm{Na})^{+}:$399.2102, found 399.2094. 


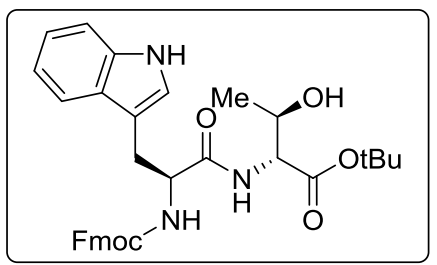

Glassy solid, 98\% yield (114.3 mg). ${ }^{1} \mathrm{H}$ NMR $\left(400 \mathrm{MHz}, \mathrm{CDCl}_{3}\right) \delta 8.20$ (s, $1 \mathrm{H}), 7.74(\mathrm{~d}, J=7.5 \mathrm{~Hz}, 2 \mathrm{H}), 7.65(\mathrm{~d}, J=7.7 \mathrm{~Hz}, 1 \mathrm{H}), 7.52-7.47$ $(\mathrm{m}, 2 \mathrm{H}), 7.38(\mathrm{t}, J=7.4 \mathrm{~Hz}, 2 \mathrm{H}), 7.31-7.24(\mathrm{~m}, 3 \mathrm{H}), 7.16(\mathrm{t}, J=7.4$ $\mathrm{Hz}, 1 \mathrm{H}), 7.10(\mathrm{t}, J=7.4 \mathrm{~Hz}, 1 \mathrm{H}), 7.03(\mathrm{~s}, 1 \mathrm{H}), 6.84(\mathrm{~d}, J=8.5 \mathrm{~Hz}, 1 \mathrm{H})$, $5.69(\mathrm{~d}, J=7.8 \mathrm{~Hz}, 1 \mathrm{H}), 4.61(\mathrm{~d}, J=5.8 \mathrm{~Hz}, 1 \mathrm{H}), 4.42(\mathrm{dd}, J=8.6,3.1$ $\mathrm{Hz}, 1 \mathrm{H}), 4.39-4.25(\mathrm{~m}, 2 \mathrm{H}), 4.21-4.09(\mathrm{~m}, 2 \mathrm{H}), 3.27(\mathrm{~d}, J=5.5 \mathrm{~Hz}$, 2H), $2.69(\mathrm{~s}, 1 \mathrm{H}), 1.43(\mathrm{~s}, 9 \mathrm{H}), 1.08(\mathrm{~d}, J=5.4 \mathrm{~Hz}, 3 \mathrm{H}) ;{ }^{13} \mathrm{C} \mathrm{NMR}\left(100 \mathrm{MHz}, \mathrm{CDCl}_{3}\right) \delta 172.0,169.5$, 156.2, 143.8, 143.7, 141.2, 136.2, 127.7, 127.1, 125.1, 123.4, 122.2, 119.9, 119.7, 118.6, 111.3, 110.2, 82.5, 68.4, 67.2, 58.2, 55.7, 47.0, 28.3, 27.9, $19.9 \mathrm{ppm}$; HRMS m/z (ESI) calcd for $\mathrm{C}_{34} \mathrm{H}_{37} \mathrm{~N}_{3} \mathrm{NaO}_{6}$ $(\mathrm{M}+\mathrm{Na})^{+}:$606.2575, found 606.2576 .

\section{Cbz-L-Trp-L-Thr-OtBu (13k)}

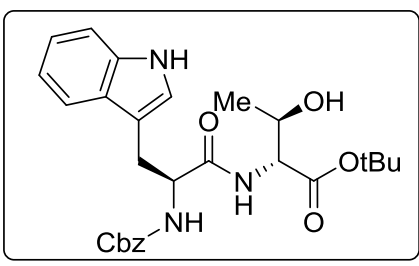

Glassy solid, $98 \%$ yield $(79.0 \mathrm{mg}) .{ }^{1} \mathrm{H}$ NMR $\left(400 \mathrm{MHz}, \mathrm{CDCl}_{3}\right) \delta 8.29$ (s, 1H), $7.59(\mathrm{~d}, J=7.5 \mathrm{~Hz}, 1 \mathrm{H}), 7.28-7.26(\mathrm{~m}, 6 \mathrm{H}), 7.14(\mathrm{t}, J=7.5$ $\mathrm{Hz}, 1 \mathrm{H}), 7.05(\mathrm{t}, J=7.4 \mathrm{~Hz}, 1 \mathrm{H}), 6.99(\mathrm{~s}, 1 \mathrm{H}), 6.88(\mathrm{~d}, J=6.2 \mathrm{~Hz}, 1 \mathrm{H})$, $5.66(\mathrm{~s}, 1 \mathrm{H}), 5.15-4.89(\mathrm{~m}, 2 \mathrm{H}), 4.59(\mathrm{~d}, J=4.9 \mathrm{~Hz}, 1 \mathrm{H}), 4.40(\mathrm{dd}, J$ $=8.6,3.2 \mathrm{~Hz}, 1 \mathrm{H}), 4.15-4.12(\mathrm{~m}, 1 \mathrm{H}), 3.24(\mathrm{~d}, J=5.3 \mathrm{~Hz}, 2 \mathrm{H}), 2.86$ (s, 1H), $1.42(\mathrm{~s}, 9 \mathrm{H}), 1.04(\mathrm{~d}, J=5.3 \mathrm{~Hz}, 3 \mathrm{H}) ;{ }^{13} \mathrm{C}$ NMR $(100 \mathrm{MHz}$, $\left.\mathrm{CDCl}_{3}\right) \delta$ 172.0, 169.6, 156.2, 136.2, 128.4, 128.3, 128.0, 128.0, 127.5, 123.4, 122.1, 119.6, 118.6, 111.2, 110.1, 82.5, 68.3, 67.0, 58.2, 55.7, 28.1, 27.9, $19.8 \mathrm{ppm} ; \mathrm{HRMS} \mathrm{m} / \mathrm{z}$ (ESI) calcd for $\mathrm{C}_{27} \mathrm{H}_{33} \mathrm{~N}_{3} \mathrm{NaO}_{6}$ $(\mathrm{M}+\mathrm{Na})^{+}:$518.2262, found 518.2260.

\section{Cbz-L-Trp-L-Tyr(tBu)-OtBu (13I)}

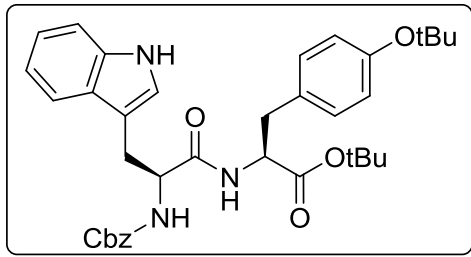

White solid, 99\% yield (121.4 mg). ${ }^{1} \mathrm{H}$ NMR (400 MHz, $\left.\mathrm{CDCl}_{3}\right) \delta$ $8.34(\mathrm{~s}, 1 \mathrm{H}), 7.65(\mathrm{~d}, J=5.9 \mathrm{~Hz}, 1 \mathrm{H}), 7.39-7.30(\mathrm{~m}, 6 \mathrm{H}), 7.18(\mathrm{t}, J$ $=7.5 \mathrm{~Hz}, 1 \mathrm{H}), 7.10(\mathrm{t}, J=7.3 \mathrm{~Hz}, 1 \mathrm{H}), 6.90(\mathrm{~s}, 1 \mathrm{H}), 6.79-6.72(\mathrm{~m}$, $4 \mathrm{H}), 6.36(\mathrm{~d}, J=7.2 \mathrm{~Hz}, 1 \mathrm{H}), 5.44(\mathrm{~d}, J=6.6 \mathrm{~Hz}, 1 \mathrm{H}), 5.11(\mathrm{q}, J=$ $12.3 \mathrm{~Hz}, 2 \mathrm{H}), 4.64-4.55(\mathrm{~m}, 2 \mathrm{H}), 3.36(\mathrm{~d}, J=12.2 \mathrm{~Hz}, 1 \mathrm{H}), 3.15$ $(\mathrm{dd}, J=14.6,6.9 \mathrm{~Hz}, 1 \mathrm{H}), 2.89(\mathrm{~d}, J=5.8 \mathrm{~Hz}, 2 \mathrm{H}), 1.35$ (s, 9H), $1.30(\mathrm{~s}, 9 \mathrm{H}) ;{ }^{13} \mathrm{C}$ NMR $(100 \mathrm{MHz}$, $\left.\mathrm{CDCl}_{3}\right) \delta 170.6,170.0,155.9,154.0,136.2,130.8,129.8,128.5,128.1,128.0,127.4,123.9,123.5$, 122.2, 119.7, 118. 7, 111.2, 110.0, 82.2, 78.4, 67.0, 55.4, 53.7, 37.3, 28.8, 28.0, 27.8 ppm; HRMS m/z (ESI) calcd for $\mathrm{C}_{36} \mathrm{H}_{43} \mathrm{~N}_{3} \mathrm{NaO}_{6}(\mathrm{M}+\mathrm{Na})^{+}$: 636.3044, found 636.3041 .

\section{Fmoc-L-Asn-L-Thr-OtBu (13m)}

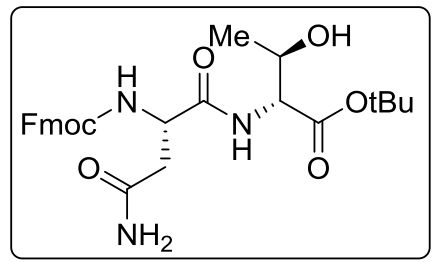

White solid, 96\% yield (98.3 mg). ${ }^{1} \mathrm{H}$ NMR (400 MHz, DMSO) $\delta 7.88$ (d, $J=7.5 \mathrm{~Hz}, 2 \mathrm{H}), 7.80-7.57(\mathrm{~m}, 4 \mathrm{H}), 7.41(\mathrm{t}, J=7.4 \mathrm{~Hz}, 2 \mathrm{H}), 7.33$ (t, $J=7.2 \mathrm{~Hz}, 3 \mathrm{H}), 6.93(\mathrm{~s}, 1 \mathrm{H}), 4.92(\mathrm{~d}, J=5.6 \mathrm{~Hz}, 1 \mathrm{H}), 4.51-4.46$ $(\mathrm{m}, 1 \mathrm{H}), 4.33-4.18(\mathrm{~m}, 3 \mathrm{H}), 4.11(\mathrm{~d}, J=7.6 \mathrm{~Hz}, 2 \mathrm{H}), 2.58(\mathrm{dd}, J=$ $15.3,4.7 \mathrm{~Hz}, 1 \mathrm{H}), 2.47-2.41(\mathrm{~m}, 1 \mathrm{H}), 1.38(\mathrm{~s}, 9 \mathrm{H}), 1.05(\mathrm{~d}, J=6.2$ $\mathrm{Hz}, 3 \mathrm{H}) .{ }^{13} \mathrm{C}$ NMR (100 MHz, DMSO) $\delta 171.8,171.5,169.6,155.9,143.9,140.8,127.7,127.2,125.4$, 120.2, 80.6, 66.5, 65.9, 58.4, 51.6, 46.7, 37.6, 27.8, 20.4 ppm; HRMS m/z (ESI) calcd for $\mathrm{C}_{27} \mathrm{H}_{33} \mathrm{~N}_{3} \mathrm{NaO}_{7}$ $(\mathrm{M}+\mathrm{Na})^{+}:$534.2211, found 534.2223.

\section{Fmoc-L-Cys-L-Thr(OtBu)-OtBu (13n)}




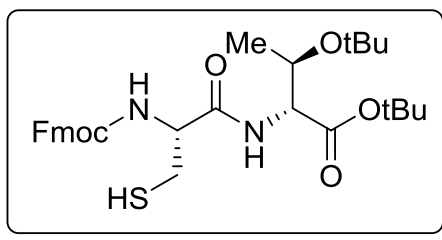

White solid, 78\% yield (78.0 mg). ${ }^{1} \mathrm{H}$ NMR $\left(400 \mathrm{MHz}, \mathrm{CDCl}_{3}\right) \delta 7.76$ $(\mathrm{d}, J=7.5 \mathrm{~Hz}, 2 \mathrm{H}), 7.60(\mathrm{~d}, J=7.3 \mathrm{~Hz}, 2 \mathrm{H}), 7.40(\mathrm{t}, J=7.4 \mathrm{~Hz}, 2 \mathrm{H})$, $7.32(\mathrm{t}, J=7.4 \mathrm{~Hz}, 2 \mathrm{H}), 6.84(\mathrm{~d}, J=7.9 \mathrm{~Hz}, 1 \mathrm{H}), 5.90(\mathrm{~d}, J=7.0 \mathrm{~Hz}$, $1 \mathrm{H}), 4.52-4.35(\mathrm{~m}, 3 \mathrm{H}), 4.31(\mathrm{~d}, J=8.8 \mathrm{~Hz}, 1 \mathrm{H}), 4.23(\mathrm{~s}, 2 \mathrm{H}), 3.18$ $-2.93(\mathrm{~m}, 1 \mathrm{H}), 2.81(\mathrm{~s}, 1 \mathrm{H}), 1.95-1.83(\mathrm{~m}, 3 \mathrm{H}), 1.47(\mathrm{~s}, 9 \mathrm{H}), 1.18$ $(\mathrm{d}, J=6.3 \mathrm{~Hz}, 3 \mathrm{H}) ;{ }^{13} \mathrm{C} \mathrm{NMR}\left(100 \mathrm{MHz}, \mathrm{CDCl}_{3}\right) \delta$ 169.7, 169.4, 155.7, 143.7, 141.3, 127.7, 127.1, 125.0, 120.0, 82.3, 74.0, 67.2, 66.9, 58.9, 47.1, 28.7, 28.1, $21.3 \mathrm{ppm}$; HRMS m/z (ESI) calcd for $\mathrm{C}_{30} \mathrm{H}_{40} \mathrm{~N}_{2} \mathrm{NaO}_{6} \mathrm{~S}(\mathrm{M}+\mathrm{Na})^{+}:$579.2499, found 579.2496.

\section{Fmoc-L-Leu-L-Leu-OtBu (13o)}

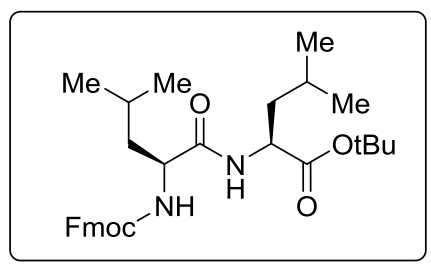

White solid, $96 \%$ yield $(65.5 \mathrm{mg}) .{ }^{1} \mathrm{H}$ NMR $\left(400 \mathrm{MHz}, \mathrm{CDCl}_{3}\right) \delta 7.75$ (d, $J=7.5 \mathrm{~Hz}, 2 \mathrm{H}), 7.58(\mathrm{~d}, J=7.3 \mathrm{~Hz}, 2 \mathrm{H}), 7.38(\mathrm{t}, J=7.2 \mathrm{~Hz}, 2 \mathrm{H})$, $7.29(\mathrm{td}, J=7.4,1.2 \mathrm{~Hz}, 2 \mathrm{H}), 6.54(\mathrm{~d}, J=7.6 \mathrm{~Hz}, 1 \mathrm{H}), 5.49(\mathrm{~d}, J=8.3$ $\mathrm{Hz}, 1 \mathrm{H}), 4.53-4.46(\mathrm{~m}, 1 \mathrm{H}), 4.43-4.33(\mathrm{~m}, 2 \mathrm{H}), 4.27(\mathrm{~d}, J=4.6 \mathrm{~Hz}$, $1 \mathrm{H}), 4.20(\mathrm{t}, J=6.9 \mathrm{~Hz}, 1 \mathrm{H}), 1.70-1.57(\mathrm{~m}, 5 \mathrm{H}), 1.46(\mathrm{~s}, 9 \mathrm{H}), 0.96-$ $0.89(\mathrm{~m}, 12 \mathrm{H}) .{ }^{13} \mathrm{C}$ NMR $\left(100 \mathrm{MHz}, \mathrm{CDCl}_{3}\right) \delta 171.8,171.7,156.1,143.8,141.2,127.6,127.0,125.0$, 119.9, 81.8, 67.0, 53.3, 51.4, 47.1, 41.6, 27.9, 26.8, 24.8, 24.6, 22.9, 22.6, 22.0, 22.0 ppm; HRMS m/z (ESI) calcd for $\mathrm{C}_{31} \mathrm{H}_{42} \mathrm{~N}_{2} \mathrm{NaO}_{5}(\mathrm{M}+\mathrm{Na})^{+}$: 545.2986, found 545.2989.

\section{Cbz-L-Ala-L-Pro-OMe (13p)}

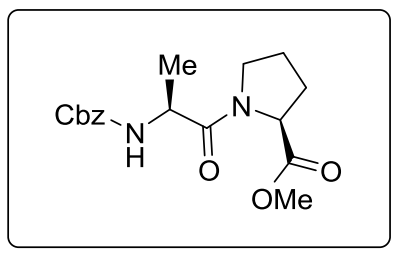

White solid, 98\% yield $(65.5 \mathrm{mg}) .{ }^{1} \mathrm{H}$ NMR $\left(400 \mathrm{MHz}, \mathrm{CDCl}_{3}\right) \delta 7.39-$ $7.28(\mathrm{~m}, 5 \mathrm{H}), 5.68(\mathrm{~d}, J=7.3 \mathrm{~Hz}, 1 \mathrm{H}), 5.08(\mathrm{~s}, 2 \mathrm{H}), 4.57-4.47(\mathrm{~m}, 2 \mathrm{H})$, $3.71(\mathrm{~s}, 3 \mathrm{H}), 3.64-3.56(\mathrm{~m}, 1 \mathrm{H}), 2.28-2.16(\mathrm{~m}, 1 \mathrm{H}), 2.15-1.84(\mathrm{~m}$, $4 \mathrm{H}), 1.38(\mathrm{~d}, J=6.8 \mathrm{~Hz}, 3 \mathrm{H}) ;{ }^{13} \mathrm{C} \mathrm{NMR}\left(100 \mathrm{MHz}, \mathrm{CDCl}_{3}\right) \delta 172.4,171.5$, 155.8, 136.6, 129.6, 128.6, 128.2, 128.1, 128.0, 66.8, 58.8, 52.3, 48.4, 46.9, 29.0, 25.0, $18.3 \mathrm{ppm}$; HRMS m/z (ESI) calcd for $\mathrm{C}_{17} \mathrm{H}_{22} \mathrm{~N}_{2} \mathrm{NaO}_{5}(\mathrm{M}+\mathrm{Na})^{+}$:

357.1421, found 357.1411.

\section{Fmoc-Aib-L-Thr-OtBu (13q)}

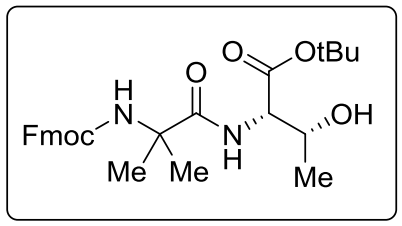

Glassy solid, 98\% yield (65.5 mg). ${ }^{1} \mathrm{H}$ NMR (400 MHz, $\left.\mathrm{CDCl}_{3}\right) \delta 7.75$ $(\mathrm{d}, J=7.5 \mathrm{~Hz}, 2 \mathrm{H}), 7.60-7.57(\mathrm{~m}, J=7.3,3.7 \mathrm{~Hz}, 2 \mathrm{H}), 7.39$ (t, $J=7.4$ $\mathrm{Hz}, 2 \mathrm{H}), 7.30$ (t, $J=7.4 \mathrm{~Hz}, 2 \mathrm{H}), 6.88(\mathrm{~d}, J=8.6 \mathrm{~Hz}, 1 \mathrm{H}), 5.59$ (s, $1 \mathrm{H})$, $4.51-4.34(\mathrm{~m}, 3 \mathrm{H}), 4.26-4.17(\mathrm{~m}, 2 \mathrm{H}), 2.63(\mathrm{~s}, 1 \mathrm{H}), 1.55(\mathrm{~d}, J=7.0$ $\mathrm{Hz}, 6 \mathrm{H}), 1.45(\mathrm{~s}, 9 \mathrm{H}), 1.20(\mathrm{~d}, J=6.2 \mathrm{~Hz}, 3 \mathrm{H}) ;{ }^{13} \mathrm{C} \mathrm{NMR}(100 \mathrm{MHz}$, $\left.\mathrm{CDCl}_{3}\right) \delta 174.4,169.9,155.2,143.8,141.3,127.6,127.0,124.9,119.9,82.4,68.6,66.7,58.0,57.0,47.1$, 27.9, 25.8, $20.1 \mathrm{ppm}$; HRMS m/z (ESI) calcd for $\mathrm{C}_{27} \mathrm{H}_{34} \mathrm{~N}_{2} \mathrm{NaO}_{6}(\mathrm{M}+\mathrm{Na})^{+}:$505.2309, found 505.2307.

\section{Fmoc-L-Val-L-Val-OtBu (13r)}

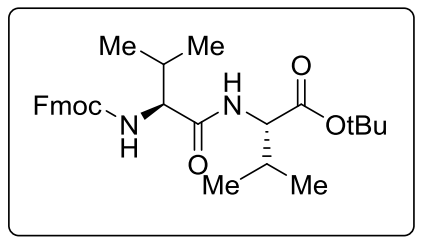

White solid, $95 \%$ yield $(93.9 \mathrm{mg}) .{ }^{1} \mathrm{H}$ NMR (400 MHz, $\left.\mathrm{CDCl}_{3}\right) \delta 7.74$ (d, $J=7.5 \mathrm{~Hz}, 2 \mathrm{H}), 7.63-7.54(\mathrm{~m}, 2 \mathrm{H}), 7.38(\mathrm{t}, J=7.1 \mathrm{~Hz}, 2 \mathrm{H}), 7.33-$ $7.26(\mathrm{~m}, 2 \mathrm{H}), 6.62(\mathrm{~s}, 1 \mathrm{H}), 5.69(\mathrm{~d}, J=8.5 \mathrm{~Hz}, 1 \mathrm{H}), 4.50-4.40(\mathrm{~m}, 2 \mathrm{H})$, $4.38-4.30(\mathrm{~m}, 1 \mathrm{H}), 4.23-4.11(\mathrm{~m}, 2 \mathrm{H}), 2.16-2.08(\mathrm{~m}, 2 \mathrm{H}), 1.45(\mathrm{~s}$, 9H), $1.01-0.94(\mathrm{~m}, 5 \mathrm{H}), 0.93-0.88(\mathrm{~m}, 7 \mathrm{H}) ;{ }^{13} \mathrm{C}$ NMR $(100 \mathrm{MHz}$,

$\left.\mathrm{CDCl}_{3}\right) \delta$ 171.2, 170.6, 156.3, 143.7, 141.2, 127.6, 127.0, 125.0, 119.8, 81.8, 67.0, 60.2, 57.6, 47.1, 31.3, 31.2, 27.9, 19.1, 18.8, 18.0, $17.7 \mathrm{ppm}$; HRMS m/z (ESI) calcd for $\mathrm{C}_{29} \mathrm{H}_{38} \mathrm{~N}_{2} \mathrm{NaO}_{5}(\mathrm{M}+\mathrm{Na})^{+}:$517.2673, found 517.2680 . 


\section{Synthesis of Protected Leu-enkephalin with Ynamide MYMsA as Coupling}

\section{Reagent}
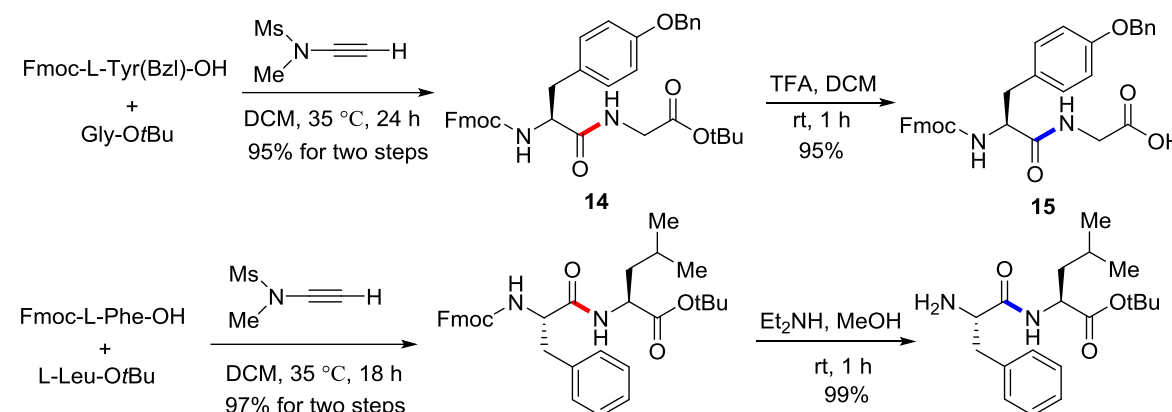

(1)

$16 \quad 17 \mathrm{Me}$
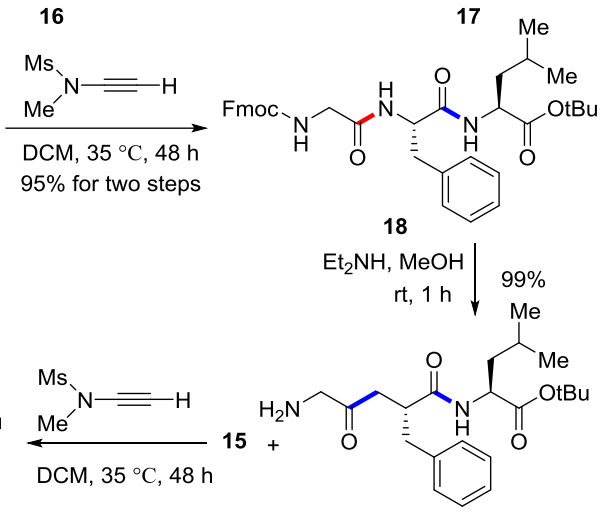

(1)

20

19

\section{Fmoc-L-Tyr(Bn)-Gly-OtBu (14):}

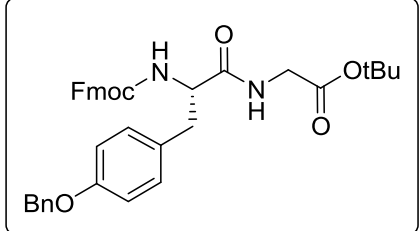

A $10 \mathrm{~mL}$ round-bottomed flask was charged with ynamide (MYMsA) (40 mg, $0.3 \mathrm{mmol}$ ), $\mathrm{CH}_{2} \mathrm{Cl}_{2}$ (3 mL) and Fmoc-L-Tyr(Bn)-OH (148 mg, $0.3 \mathrm{mmol})$. The reaction mixture was stirred at room temperature for 40 min under air until starting material was fully consumed by TLC. HGly-OtBu (47 mg, $0.36 \mathrm{mmol}$ ) was added and the reaction mixture was stirred at $35{ }^{\circ} \mathrm{C}$ for $24 \mathrm{~h}$ under air until $\alpha$-acyloxyenamide was fully consumed by TLC. The reaction mixture was concentrated and purified by silica gel chromatography to afford the respective peptide $\mathbf{1 4}(95 \%, 173 \mathrm{mg})$.

${ }^{1} \mathrm{H}$ NMR (400 MHz, CDCl3) $\delta 7.76(\mathrm{~d}, \mathrm{~J}=7.5 \mathrm{~Hz}, 2 \mathrm{H}), 7.57-7.50(\mathrm{~m}, 2 \mathrm{H}), 7.38(\mathrm{q}, \mathrm{J}=7.9 \mathrm{~Hz}, 6 \mathrm{H})$, $7.34-7.27$ (m, 3H), 7.11 (d, J = 4.6 Hz, 2H), 6.89 (d, J = 8.4 Hz, 2H), 6.40 (s, 1H), 5.43 (s, 1H), 4.99 (s, 2H), $4.50-4.27(\mathrm{~m}, 3 \mathrm{H}), 4.18(\mathrm{t}, \mathrm{J}=6.8 \mathrm{~Hz}, 1 \mathrm{H}), 3.88(\mathrm{dd}, \mathrm{J}=39.9,17.5 \mathrm{~Hz}, 2 \mathrm{H}), 3.05(\mathrm{~s}, 2 \mathrm{H}), 1.46$ (s, 9H); ${ }^{13} \mathrm{C}$ NMR (100 MHz, CDCl3) $\delta 171.1,168.6,158.1,143.9,141.4,137.1,130.5,128.7,128.1,127.8$, 127.6, 127.2, 125.2, 120.1, 115.2, 82.5, 70.2, 67.2, 56.4, 47.3, 42.1, 37.8, 28.1 ppm; HRMS m/z (ESI) calcd for $\mathrm{C}_{37} \mathrm{H}_{38} \mathrm{~N}_{2} \mathrm{NaO}_{6}(\mathrm{M}+\mathrm{Na})+:$ 629.2622, found 629.2621 .

\section{Fmoc-L-Tyr(Bn)-Gly-OH (15)}

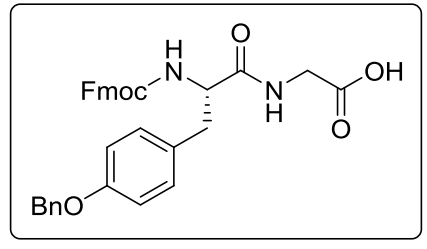

Fmoc-L-Tyr(Bn)-Gly-OtBu (14, $182 \mathrm{mg}, 0.3 \mathrm{mmol})$ was dissolved in 2 $\mathrm{mL}$ of $\mathrm{CH}_{2} \mathrm{Cl}_{2}$ and TFA ( $2 \mathrm{~mL}$ ) was added dropwise and stirred at room temperature for $1 \mathrm{~h}$. And the solvent was removed under reduced pressure and the addition of ether $(3 \mathrm{~mL})$ to the residue led to the precipitation of a white crystal, which was washed with ether and dried 
in vacuo to give product $15(157 \mathrm{mg}, 95 \%)$.

${ }^{1} \mathrm{H}$ NMR (400 MHz, DMSO) $\delta 8.31(\mathrm{~s}, 1 \mathrm{H}), 7.87(\mathrm{~d}, J=7.5 \mathrm{~Hz}, 2 \mathrm{H}), 7.71-7.51(\mathrm{~m}, 3 \mathrm{H}), 7.43-7.23$ $(\mathrm{m}, 11 \mathrm{H}), 6.88(\mathrm{~d}, J=8.4 \mathrm{~Hz}, 2 \mathrm{H}), 5.06-4.93(\mathrm{~m}, 2 \mathrm{H}), 4.26(\mathrm{t}, J=9.6 \mathrm{~Hz}, 1 \mathrm{H}), 4.17-4.11(\mathrm{~m}, 8.2 \mathrm{~Hz}$, $3 \mathrm{H}), 3.85-3.77(\mathrm{~m}, 2 \mathrm{H}), 2.99(\mathrm{~d}, J=13.6 \mathrm{~Hz}, 1 \mathrm{H}), 2.77-2.66(\mathrm{~m}, 1 \mathrm{H}) ;{ }^{13} \mathrm{C}$ NMR $(100 \mathrm{MHz}, \mathrm{DMSO})$ $\delta 172.4,171.6,157.4,156.3,144.2,141.1,137.7,130.8,130.7,128.8,128.2$, 128.1, 127.5, 125.9, 125.8, $120.5,114.9,69.7,66.2,56.8,47.1,41.3,37.2 \mathrm{ppm}$; HRMS m/z (ESI) calcd for $\mathrm{C}_{33} \mathrm{H}_{30} \mathrm{~N}_{2} \mathrm{NaO}_{6}(\mathrm{M}+\mathrm{Na})^{+}$: 573.1996, found 573.1998 .

\section{Fmoc-L-Phe-L-Leu-OtBu (16)}

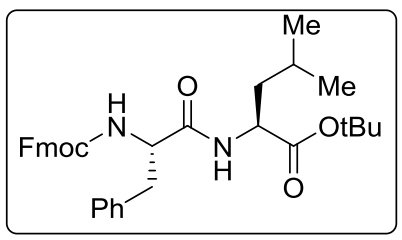

A $10 \mathrm{~mL}$ round-bottomed flask was charged with ynamide (MYMsA) (40 $\mathrm{mg}, 0.3 \mathrm{mmol}$ ), $\mathrm{CH}_{2} \mathrm{Cl}_{2}$ (3 mL) and Fmoc-L-Phe-OH (116 mg, $0.3 \mathrm{mmol}$ ). The reaction mixture was stirred at room temperature for $40 \mathrm{~min}$ under air until starting material was fully consumed by TLC. H-L-Leu-OtBu (47 mg, $0.36 \mathrm{mmol}$ ) was added and the reaction mixture was stirred at $35{ }^{\circ} \mathrm{C}$ for $24 \mathrm{~h}$ under air until $\alpha$-acyloxyenamide was fully consumed by TLC. The reaction mixture was concentrated and purified by silica gel chromatography to afford the respective peptide $\mathbf{1 6}$ (97\%, 162 $\mathrm{mg})$.

${ }^{1} \mathrm{H}$ NMR $\left(400 \mathrm{MHz}, \mathrm{CDCl}_{3}\right) \delta 7.77(\mathrm{~d}, J=7.5 \mathrm{~Hz}, 2 \mathrm{H}), 7.55(\mathrm{t}, J=7.8 \mathrm{~Hz}, 2 \mathrm{H}), 7.40(\mathrm{t}, J=7.5 \mathrm{~Hz}, 2 \mathrm{H})$, $7.35-7.15(\mathrm{~m}, 7 \mathrm{H}), 6.59(\mathrm{~s}, 1 \mathrm{H}), 5.63(\mathrm{~d}, J=7.1 \mathrm{~Hz}, 1 \mathrm{H}), 4.57(\mathrm{~d}, J=5.7 \mathrm{~Hz}, 1 \mathrm{H}), 4.52-4.41(\mathrm{~m}, 2 \mathrm{H})$, $4.33-4.23(\mathrm{~m}, 1 \mathrm{H}), 4.18(\mathrm{t}, J=7.0 \mathrm{~Hz}, 1 \mathrm{H}), 3.11(\mathrm{~d}, J=5.3 \mathrm{~Hz}, 2 \mathrm{H}), 1.68-1.50(\mathrm{~m}, 3 \mathrm{H}), 1.47(\mathrm{~s}, 9 \mathrm{H})$, $0.90(\mathrm{t}, J=5.1 \mathrm{~Hz}, 6 \mathrm{H}) ;{ }^{13} \mathrm{C} \mathrm{NMR}\left(100 \mathrm{MHz}, \mathrm{CDCl}_{3}\right) \delta 171.5,170.4,155.8,143.70,143.6,141.2,136.2$, 129.3, 128.5, 127.6, 127.0, 126.9, 125.0, 124.9, 119.8, 81.8, 67.0, 55.8, 51.4, 47.0, 41.7, 38.5, 27.9, 24.7, 22.6, $22.0 \mathrm{ppm}$; HRMS m/z (ESI) calcd for $\mathrm{C}_{34} \mathrm{H}_{40} \mathrm{~N}_{2} \mathrm{NaO}_{5}(\mathrm{M}+\mathrm{Na})^{+}:$579.2829, found 579.2830.

\section{H-L-Phe-L-Leu-OtBu (17)}

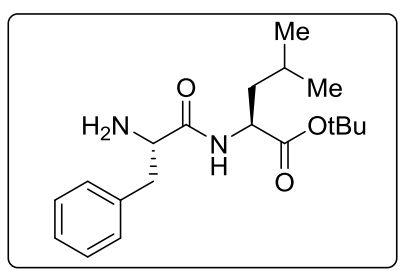

Fmoc-L-Phe-L-Leu-OtBu 16 (167 mg, $0.3 \mathrm{mmol})$ was dissolved in $2 \mathrm{~mL}$ of $\mathrm{MeOH}$ and $\mathrm{Et}_{2} \mathrm{NH}(1 \mathrm{~mL})$ was added dropwise and stirred at room temperature for $1 \mathrm{~h}$. And the solvent was removed under reduced pressure to afford the crude product, which was then purified by silica gel flash chromatography to give the pure dipeptide product $17(99 \%, 100 \mathrm{mg})$. ${ }^{1} \mathrm{H}$ NMR $\left(400 \mathrm{MHz}, \mathrm{CDCl}_{3}\right) \delta 7.64(\mathrm{~d}, J=8.4 \mathrm{~Hz}, 1 \mathrm{H}), 7.33-7.21(\mathrm{~m}$, $5 \mathrm{H}), 5.29(\mathrm{~s}, 1 \mathrm{H}), 4.49(\mathrm{td}, J=8.7,5.0 \mathrm{~Hz}, 1 \mathrm{H}), 3.65(\mathrm{~d}, J=13.0 \mathrm{~Hz}, 1 \mathrm{H}), 3.23(\mathrm{dd}, J=13.7,3.9 \mathrm{~Hz}$, $1 \mathrm{H}), 2.84-2.67(\mathrm{~m}, 1 \mathrm{H}), 1.86(\mathrm{~s}, 2 \mathrm{H}), 1.63-1.57(\mathrm{~m}, 2 \mathrm{H}), 1.46(\mathrm{~s}, 9 \mathrm{H}), 0.94(\mathrm{dd}, J=6.0,2.0 \mathrm{~Hz}, 6 \mathrm{H})$; ${ }^{13} \mathrm{C}$ NMR $\left(100 \mathrm{MHz}, \mathrm{CDCl}_{3}\right) \delta 174.1,172.2,137.8,129.4,128.7,126.9,81.8,56.4,51.1,41.8,41.0$, 28.1, 25.0, 22.9, $22.1 \mathrm{ppm}$; HRMS m/z (ESI) calcd for $\mathrm{C}_{19} \mathrm{H}_{30} \mathrm{~N}_{2} \mathrm{NaO}_{3}(\mathrm{M}+\mathrm{Na})^{+}$: 357.2149, found 357.2154 .

\section{Fmoc-Gly-L-Phe-L-Leu-OtBu (18)}

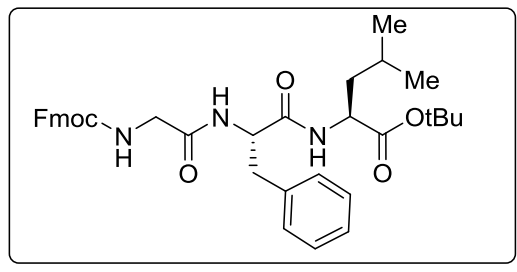

A $10 \mathrm{~mL}$ round-bottomed flask was charged with ynamide (MYMsA) (40 mg, $0.3 \mathrm{mmol}), \mathrm{CH}_{2} \mathrm{Cl}_{2}(3 \mathrm{~mL})$ and Fmoc-Gly$\mathrm{OH}(89 \mathrm{mg}, 0.3 \mathrm{mmol})$. The reaction mixture was stirred at room temperature for 40 min under air until starting material was fully consumed by TLC, H-L-Phe-L-Leu-OtBu (17, $120 \mathrm{mg}, 0.36$ mmol) was added and the reaction mixture was stirred at $35{ }^{\circ} \mathrm{C}$ for $24 \mathrm{~h}$ under air until $\alpha$-acyloxyenamide was fully consumed by TLC. The reaction mixture was concentrated and purified by silica gel chromatography to afford the respective peptide $\mathbf{1 8}$ (95\%, 175 
$\mathrm{mg})$.

${ }^{1} \mathrm{H} \mathrm{NMR}\left(400 \mathrm{MHz}, \mathrm{CDCl}_{3}\right) \delta 7.76(\mathrm{~d}, J=7.5 \mathrm{~Hz}, 2 \mathrm{H}), 7.58(\mathrm{~d}, J=7.3 \mathrm{~Hz}, 2 \mathrm{H}), 7.40(\mathrm{t}, J=7.4 \mathrm{~Hz}, 2 \mathrm{H})$, $7.32-7.16(\mathrm{~m}, 7 \mathrm{H}), 6.76(\mathrm{~s}, 1 \mathrm{H}), 6.40(\mathrm{~s}, 1 \mathrm{H}), 5.56(\mathrm{~s}, 1 \mathrm{H}), 4.79-4.63(\mathrm{~m}, 1 \mathrm{H}), 4.38(\mathrm{~d}, J=7.3 \mathrm{~Hz}$, 2H), $4.20(\mathrm{t}, J=7.0 \mathrm{~Hz}, 1 \mathrm{H}), 3.85(\mathrm{~d}, J=5.2 \mathrm{~Hz}, 1 \mathrm{H}), 3.08(\mathrm{~d}, J=6.5 \mathrm{~Hz}, 2 \mathrm{H}), 1.83(\mathrm{~s}, 2 \mathrm{H}), 1.44(\mathrm{~s}$, $9 \mathrm{H}), 1.26(\mathrm{~s}, 3 \mathrm{H}), 0.88(\mathrm{~d}, J=5.1 \mathrm{~Hz}, 6 \mathrm{H}) ;{ }^{13} \mathrm{C} \mathrm{NMR}\left(100 \mathrm{MHz}, \mathrm{CDCl}_{3}\right) \delta 171.5,170.0,168.7,156.6$, 143.8, 141.3, 136.1, 129.3, 128.6, 127.7, 127.1, 125.1, 112.0, 82.0, 67.3, 54.3, 51.6, 47.1, 44.5, 41.7, 38.2, 28.0, 24.8, 22.6, $22.1 \mathrm{ppm}$; HRMS m/z (ESI) calcd for $\mathrm{C}_{36} \mathrm{H}_{43} \mathrm{~N}_{3} \mathrm{NaO}_{6}(\mathrm{M}+\mathrm{Na})^{+}$: 636.3044, found 636.3043 .

\section{H-Gly-L-Phe-L-Leu-OtBu (19)}

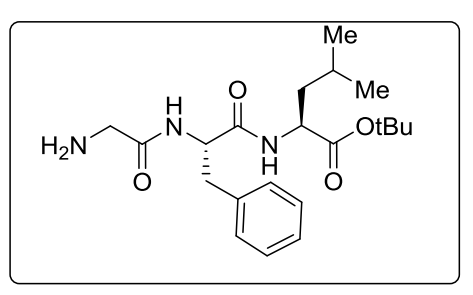

Fmoc-Gly-L-Phe-L-Leu-OtBu 18 (184 mg, $0.3 \mathrm{mmol})$ was dissolved in $2 \mathrm{~mL}$ of $\mathrm{MeOH}$ and $\mathrm{Et}_{2} \mathrm{NH}(1 \mathrm{~mL})$ was added dropwise and stirred at room temperature for $1 \mathrm{~h}$. And the solvent was removed under reduced pressure to afford the crude product, which was then purified by silica gel flash chromatography to give the pure dipeptide product $19(99 \%, 116 \mathrm{mg})$.

${ }^{1} \mathrm{H}$ NMR $\left(400 \mathrm{MHz}, \mathrm{CDCl}_{3}\right) \delta 7.70(\mathrm{~d}, J=7.9 \mathrm{~Hz}, 1 \mathrm{H}), 7.29-7.19(\mathrm{~m}, 5 \mathrm{H}), 6.54(\mathrm{~s}, 1 \mathrm{H}), 4.69(\mathrm{dd}, J=$ 14.8, 7.2 Hz, 1H), $4.43-4.37(\mathrm{~m}, 1 \mathrm{H}), 3.30(\mathrm{~s}, 1 \mathrm{H}), 3.17-3.04(\mathrm{~m}, 2 \mathrm{H}), 1.80(\mathrm{~s}, 2 \mathrm{H}), 1.60-1.53(\mathrm{~m}$, $2 \mathrm{H}), 1.44(\mathrm{~s}, 9 \mathrm{H}), 1.31-1.25(\mathrm{~m}, 1 \mathrm{H}), 0.90(\mathrm{dd}, J=6.0,2.8 \mathrm{~Hz}, 6 \mathrm{H}) ;{ }^{13} \mathrm{C} \mathrm{NMR}\left(100 \mathrm{MHz}, \mathrm{CDCl}_{3}\right) \delta$ 173.0, 171.7, 170.6, 136.8, 129.5, 128.6, 127.0, 82.0, 54.2, 51.7, 44.8, 41.8, 38.1, 28.1, 25.0, 22.8, 22.3 ppm; HRMS m/z (ESI) calcd for $\mathrm{C}_{21} \mathrm{H}_{34} \mathrm{~N}_{3} \mathrm{O}_{4}(\mathrm{M}+\mathrm{H})^{+}:$392.2549, found 392.2547.

Fmoc-L-Tyr(Bn)-Gly-Gly-L-Phe-L-Leu-OtBu (20)

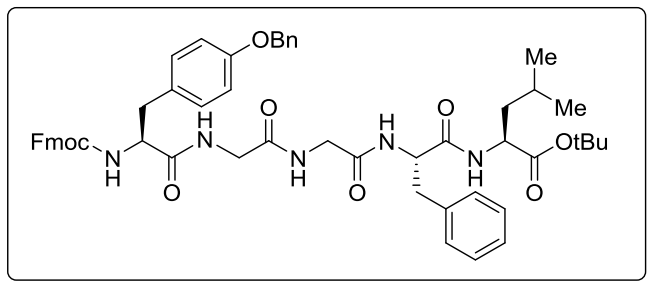

A $10 \mathrm{~mL}$ round-bottomed flask was charged with ynamide (MYMsA) (40 mg, $0.3 \mathrm{mmol}), \mathrm{CH}_{2} \mathrm{Cl}_{2}(3 \mathrm{~mL})$ and Fmoc-L-Tyr(Bn)-Gly-OH (15, $165 \mathrm{mg}, 0.3 \mathrm{mmol})$. The reaction mixture was stirred at room temperature for 40 min under air until starting material was fully consumed by TLC. H-Gly-L-Phe-L-Leu-OtBu (19, 140 $\mathrm{mg}, 0.36 \mathrm{mmol}$ ) was added and the reaction mixture was stirred at $35{ }^{\circ} \mathrm{C}$ for $48 \mathrm{~h}$ under air until $\alpha$ acyloxyenamide was fully consumed by TLC. The reaction mixture was concentrated and purified by silica gel chromatography to afford the respective peptide $\mathbf{2 0}(70 \%, 194 \mathrm{mg})$.

${ }^{1} \mathrm{H}$ NMR $\left(400 \mathrm{MHz}, \mathrm{CDCl}_{3}\right) \delta$ 8.08-8.03 (m, 1H), 7.81-7.69 (m, 4H), 7.63-7.61 (m, 1H), 7.54-7.52 $(\mathrm{m}, 1 \mathrm{H}), 7.36-7.20(\mathrm{~m}, 9 \mathrm{H}), 7.09-6.96(\mathrm{~m}, 7 \mathrm{H}), 6.76-6.74(\mathrm{~m}, 2 \mathrm{H}), 5.22-5.17(\mathrm{~m}, 1 \mathrm{H}), 4.80(\mathrm{~s}, 2 \mathrm{H}), 4.54-$ $4.35(\mathrm{~m}, 3 \mathrm{H}), 4.18-4.07(\mathrm{~m}, 4 \mathrm{H}), 3.09-2.88(\mathrm{~m}, 4 \mathrm{H}), 1.82-1.76(\mathrm{~m}, 2 \mathrm{H}), 1.53-1.40(\mathrm{~m}, 4 \mathrm{H}), 1.26(\mathrm{~s}, 9 \mathrm{H})$, 0.80-0.78 (m, 6H); ${ }^{13} \mathrm{C}$ NMR (100 MHz, $\left.\mathrm{CDCl}_{3}\right) \delta 172.0,170.6,168.8,168.3,157.9,156.6,144.1,143.8$, 141.4, 137.1, 136.7, 130.6, 129.7, 128.6, 128.4, 128.0, 127.80, 127.6, 127.2, 126.9, 125.4, 120.0, 114.8, 81.7, 70.0, 67.5, 56.0, 54.2, 51.5, 47.1, 43.6, 43.4, 42.0, 39.6, 39.1, 28.0, 24.9, 22.6, 22.4 ppm; HRMS $\mathrm{m} / \mathrm{z}(\mathrm{ESI})$ calcd for $\mathrm{C}_{54} \mathrm{H}_{61} \mathrm{~N}_{5} \mathrm{NaO}_{9}(\mathrm{M}+\mathrm{Na})^{+}:$946.4361, found 946.4406. 


\section{NMR Spectrums}

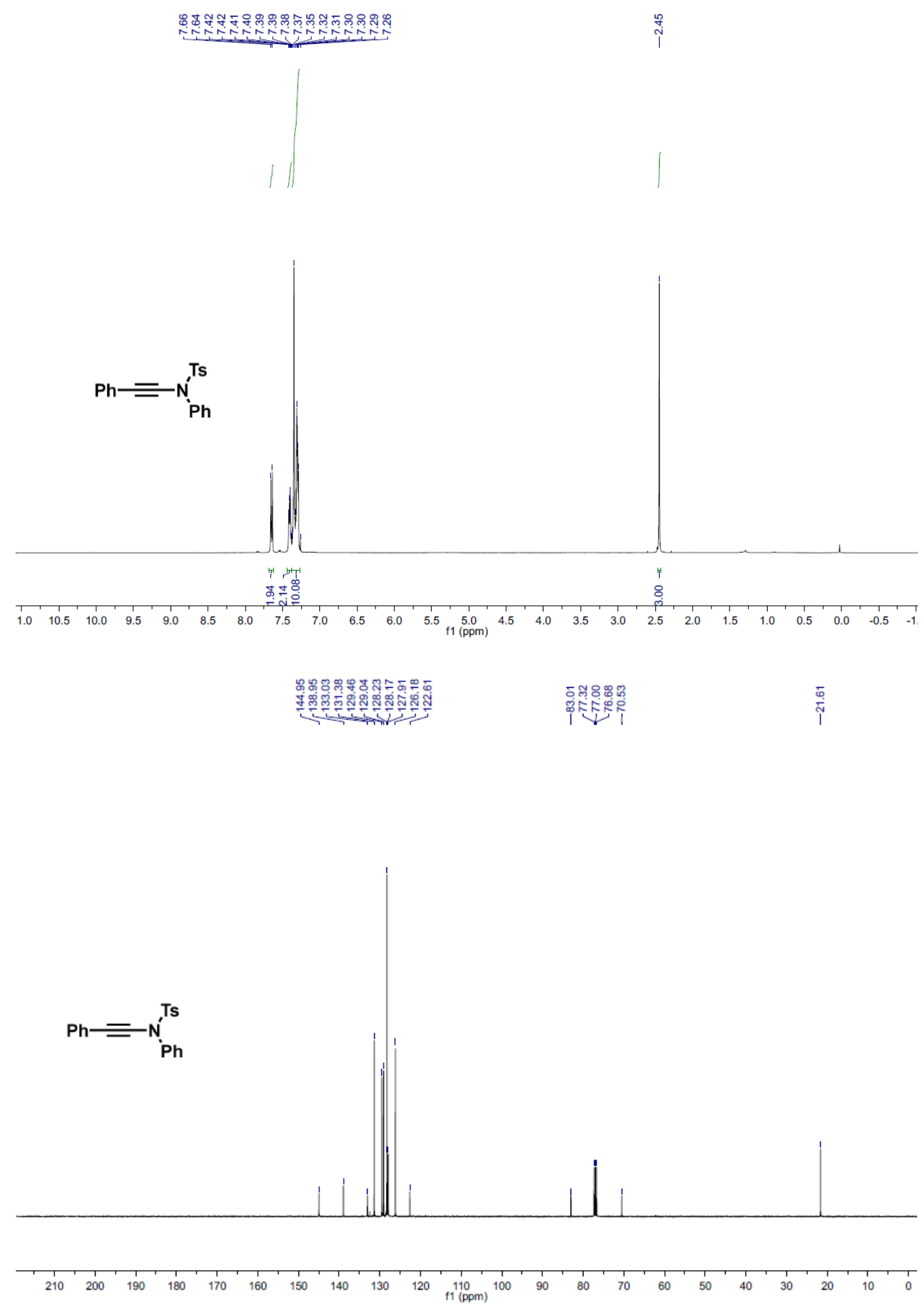




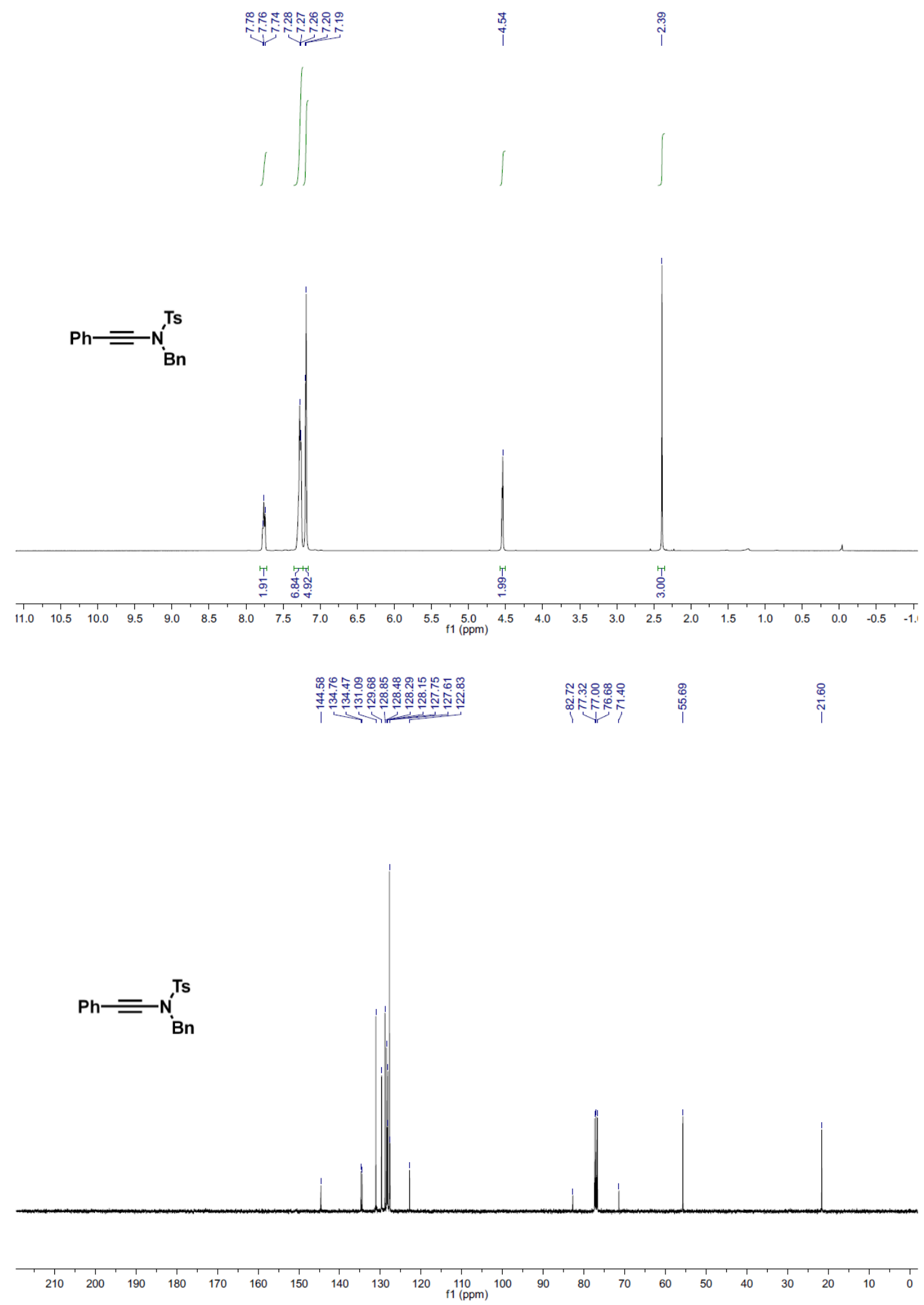



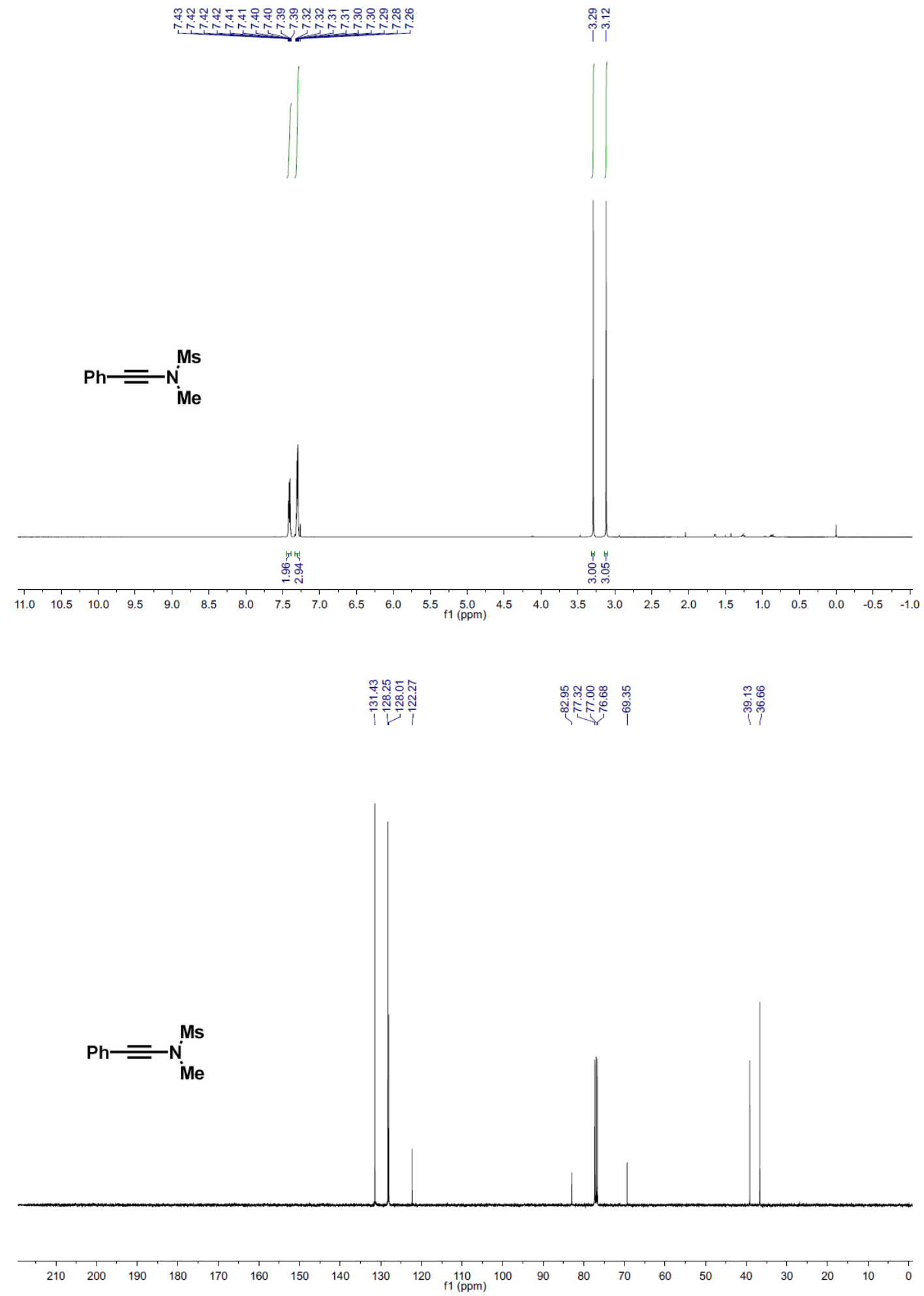

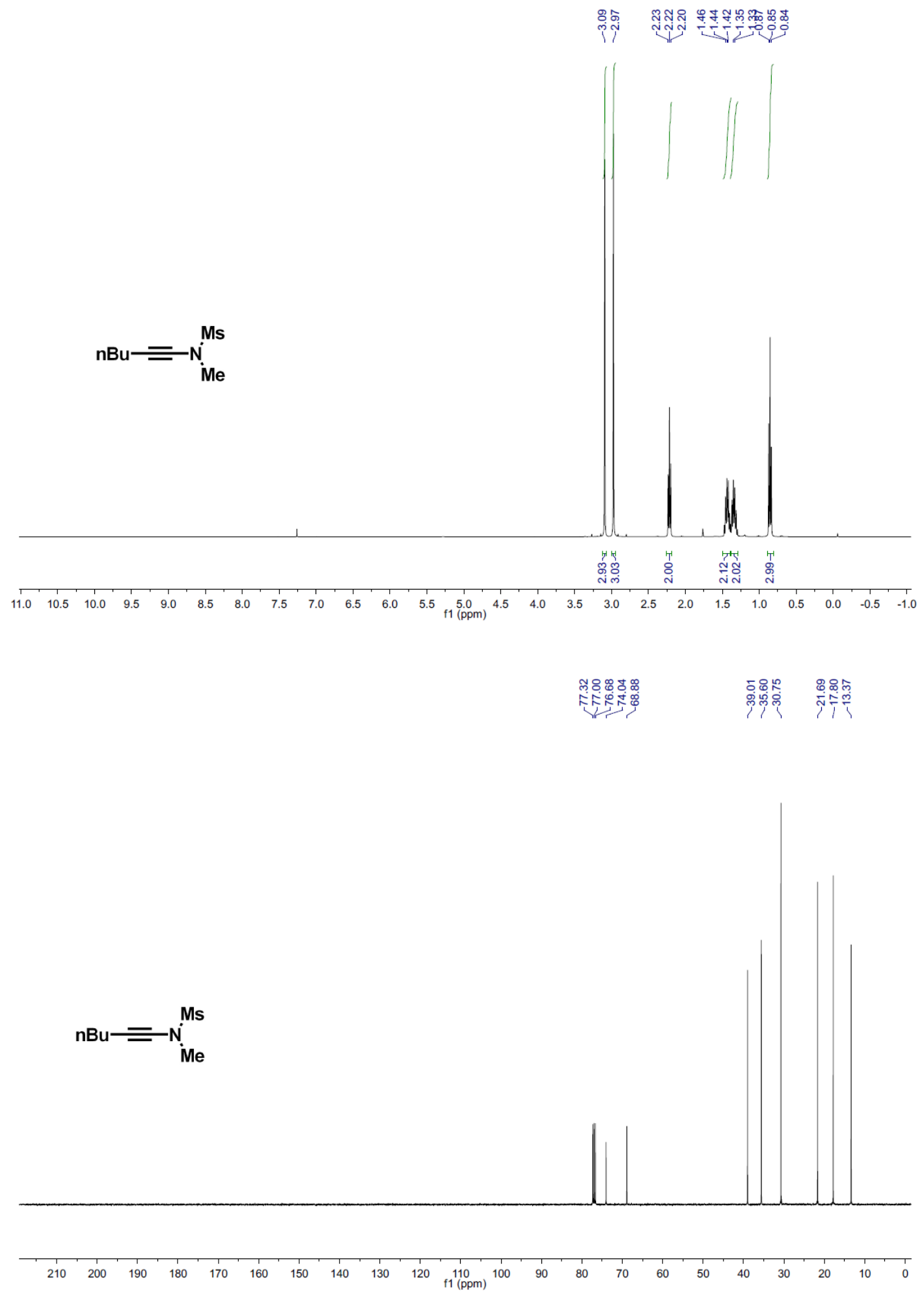

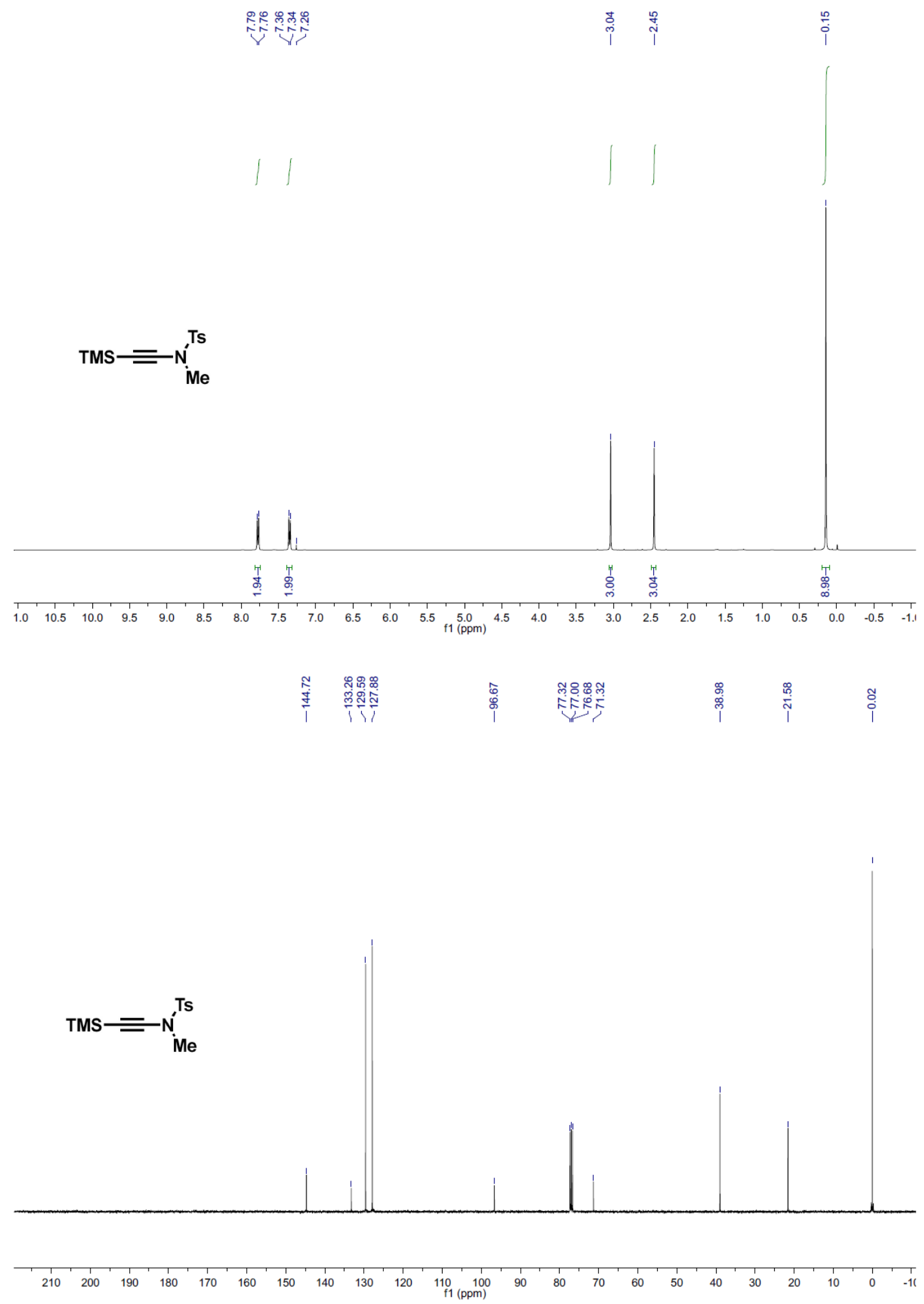


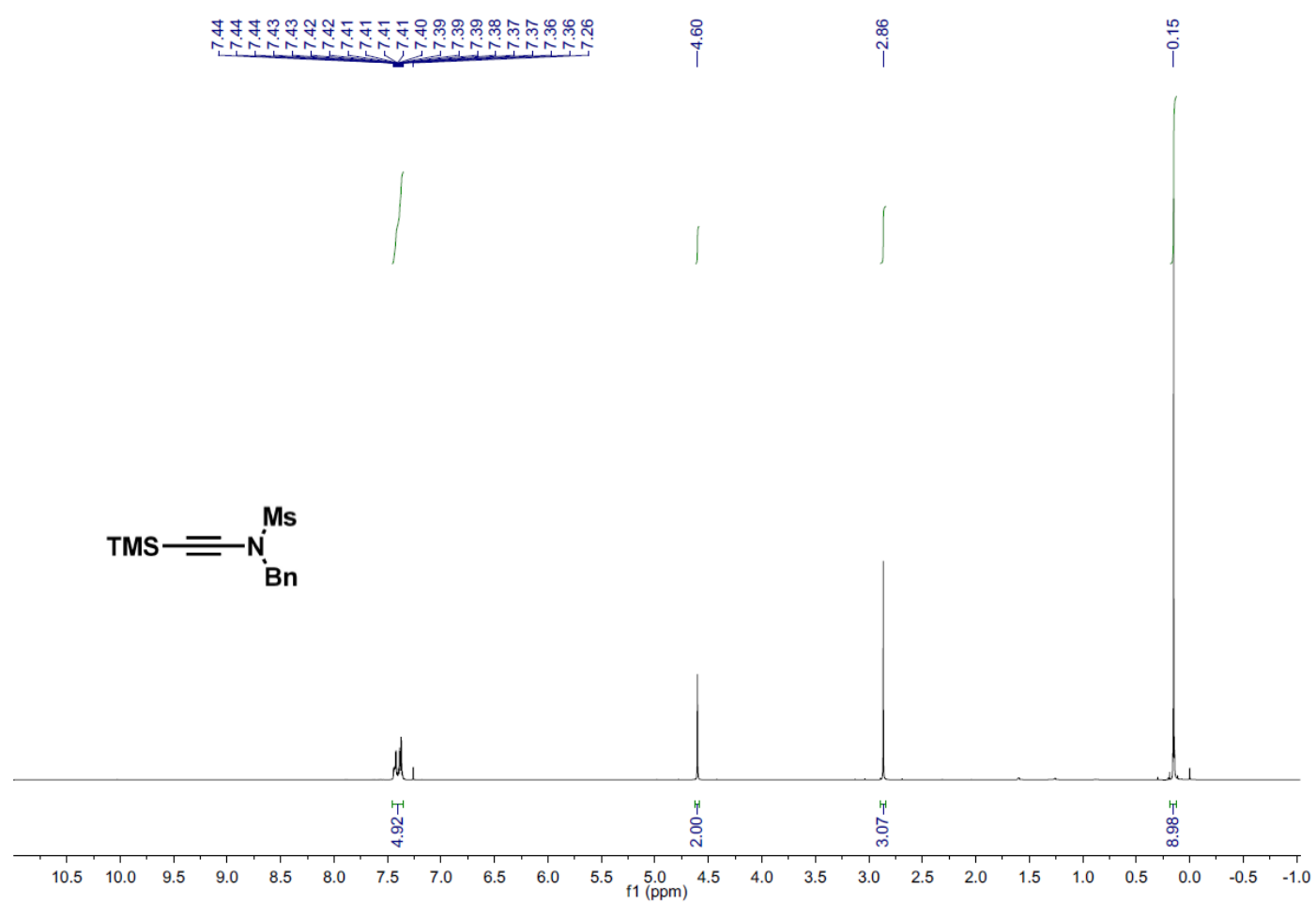

\begin{tabular}{|c|c|c|c|c|}
\hline 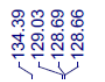 & 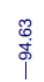 & 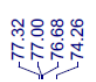 & 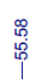 & $\begin{array}{l}\infty \\
\infty \\
\infty \\
\prod\end{array}$ \\
\hline
\end{tabular}

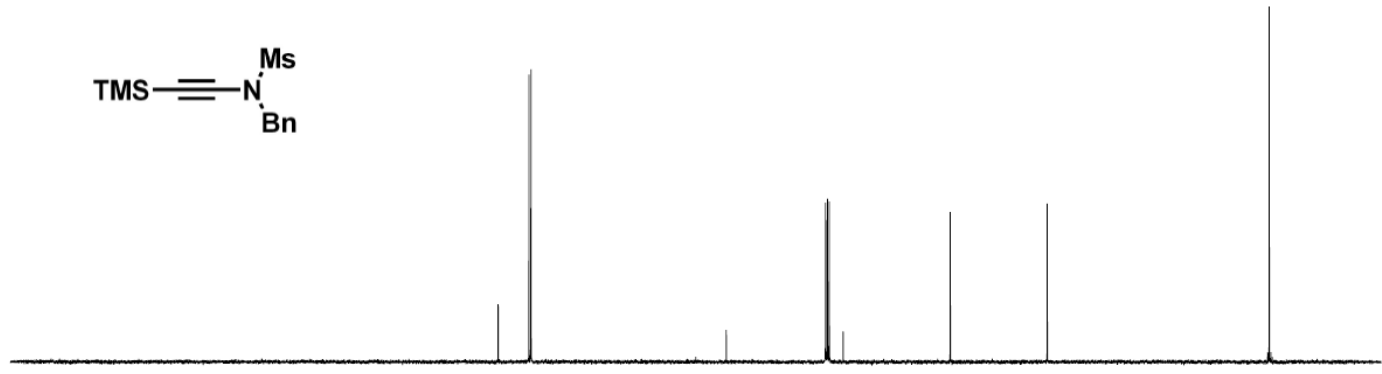

$\begin{array}{lllllllllllllllllllllllllllll}210 & 200 & 190 & 180 & 170 & 160 & 150 & 140 & 130 & 120 & 110 & 100 & 90 & 8 & 70 & 60 & 50 & 40 & 30 & 20 & 10 & 0 & -10\end{array}$ 


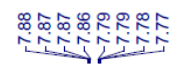

II
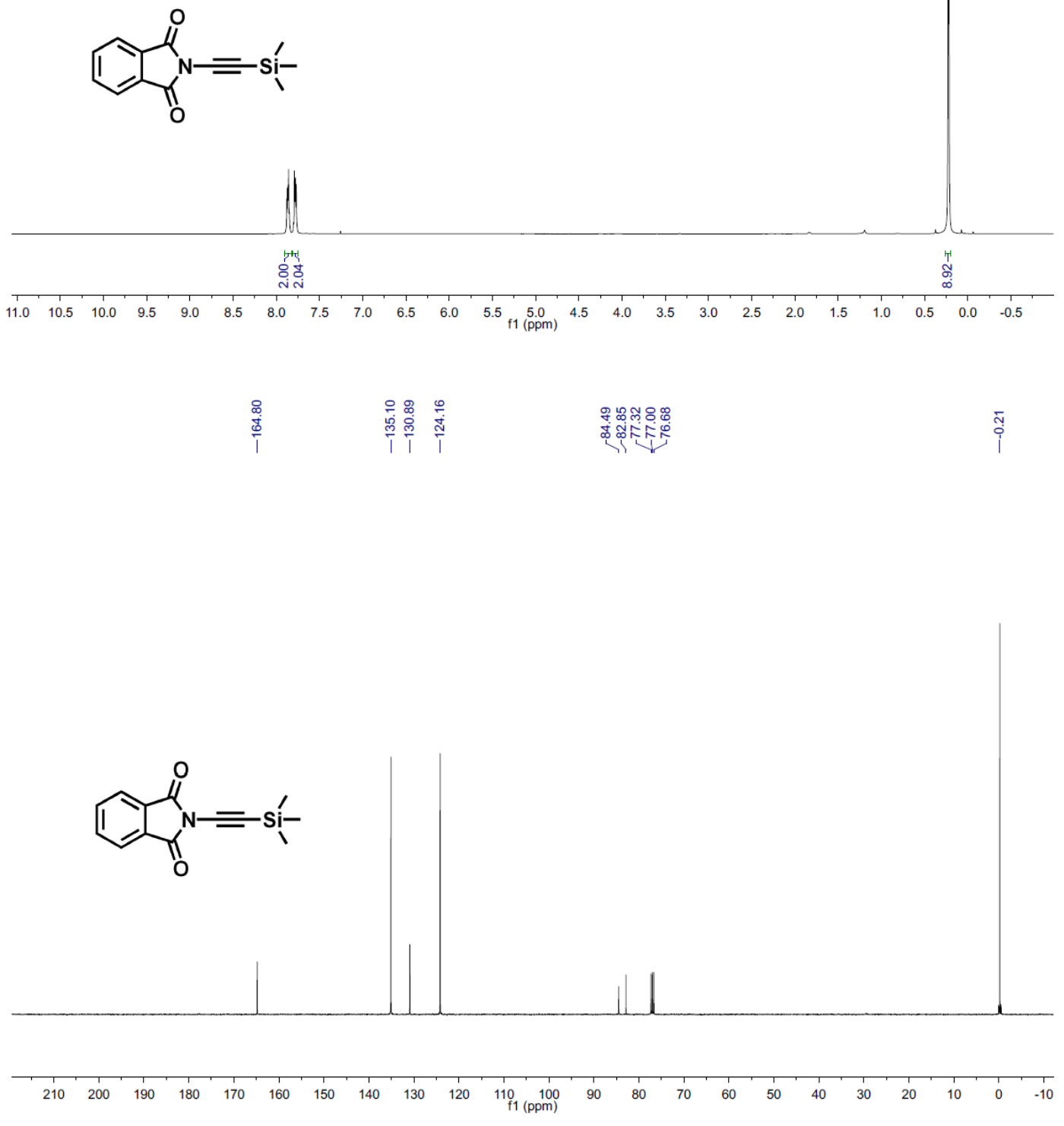

S48 


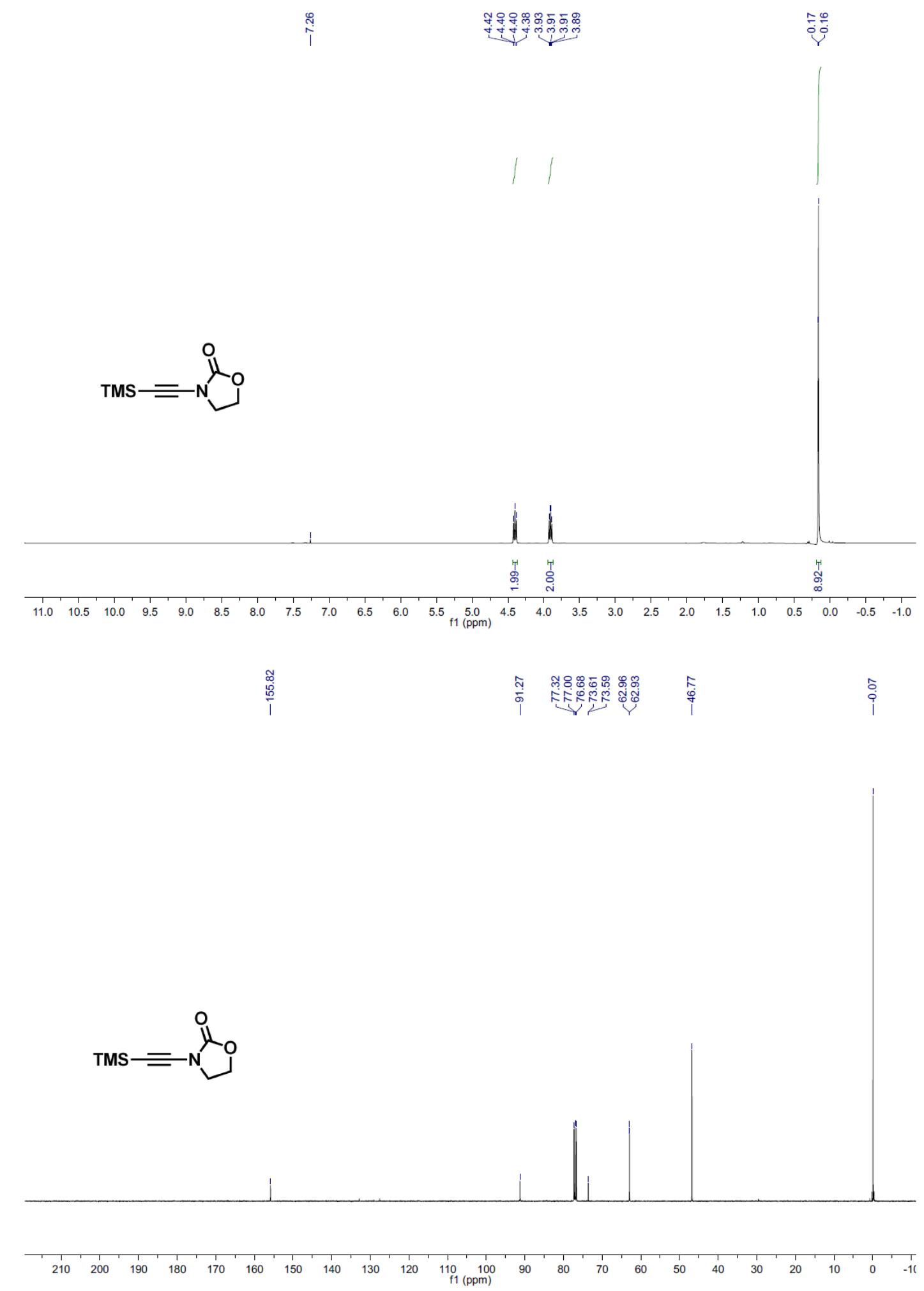




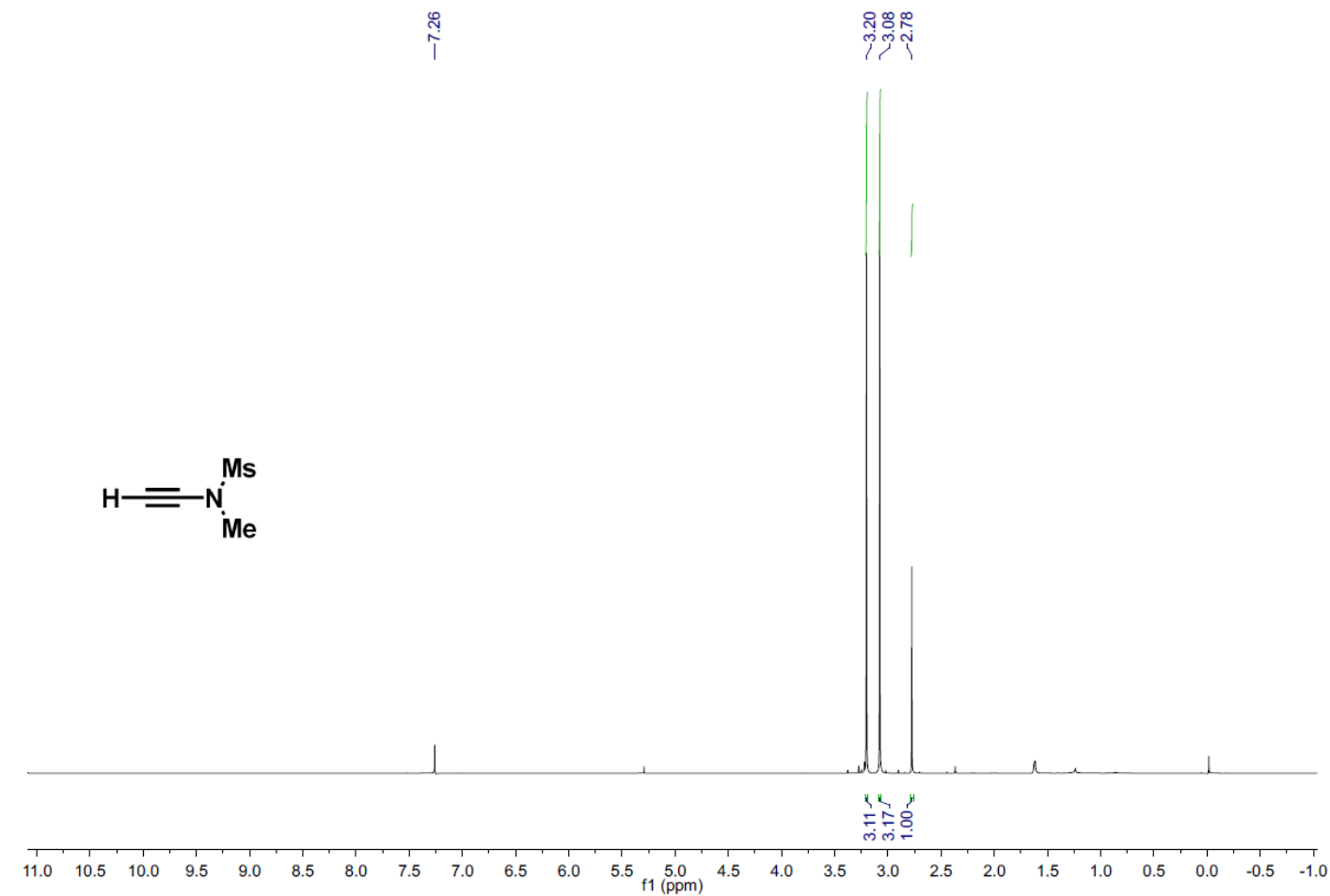

$$
\mathrm{H}=-\mathrm{N}_{\mathrm{Me}}^{\mathrm{Ms}}
$$

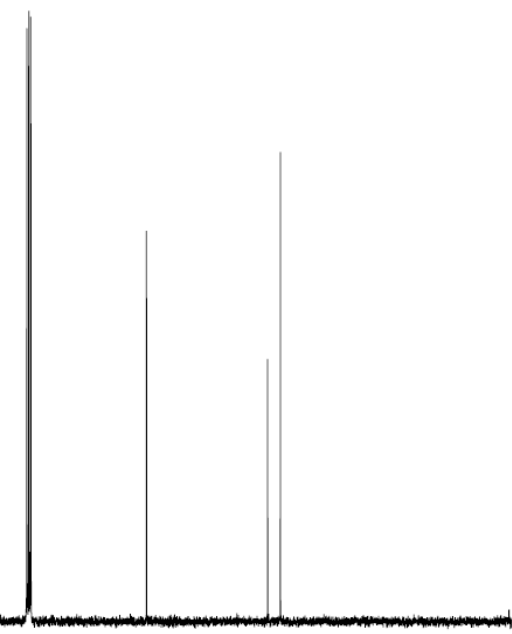

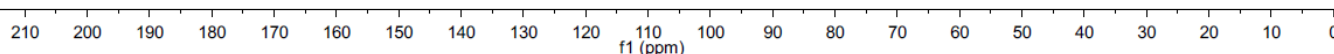




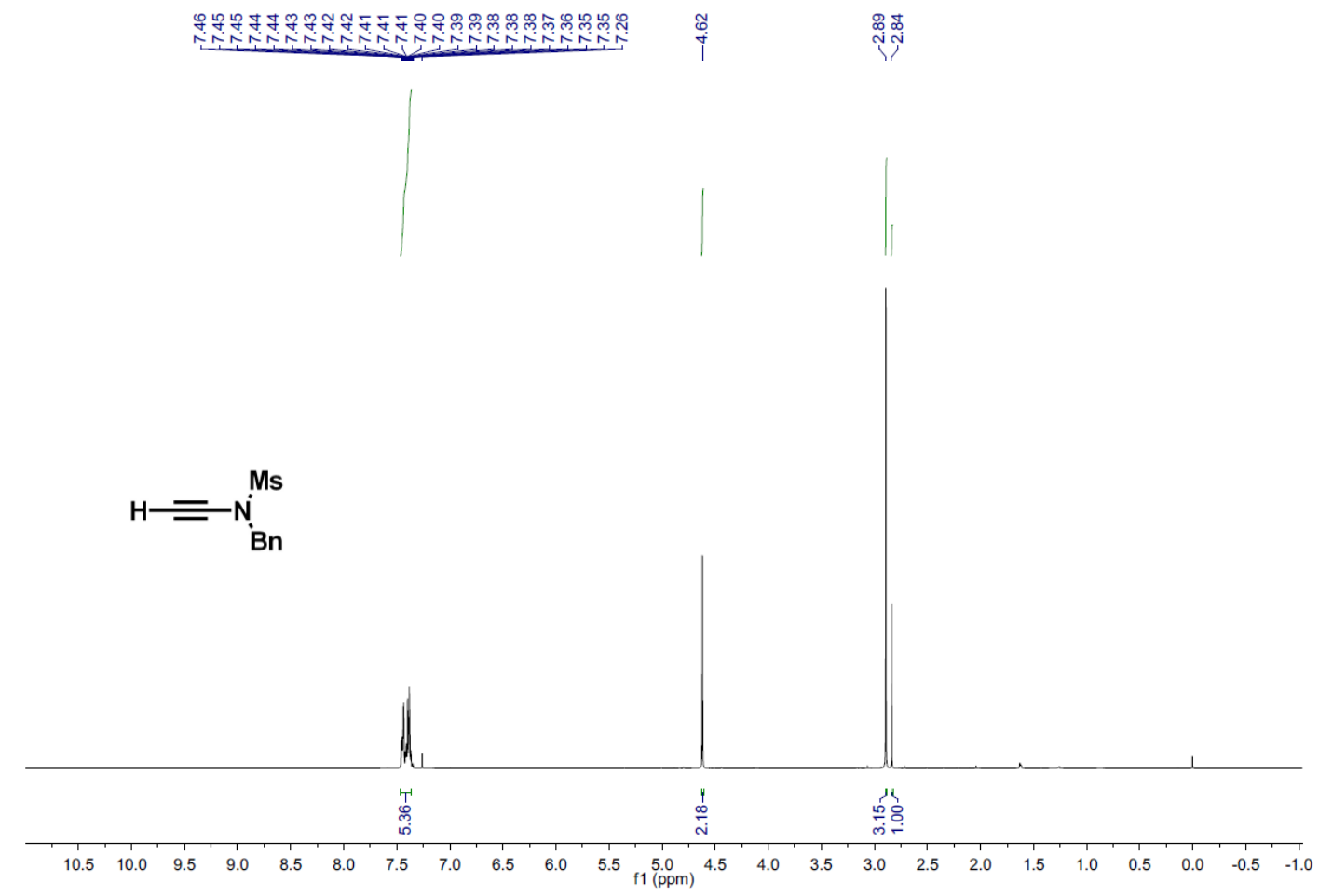

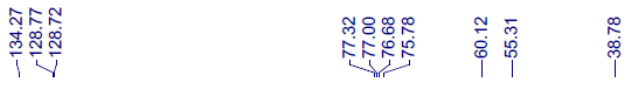

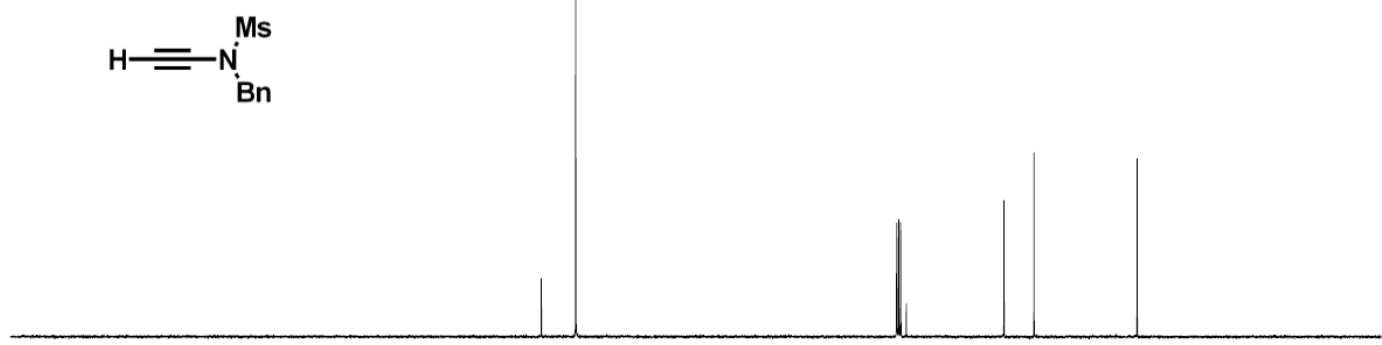

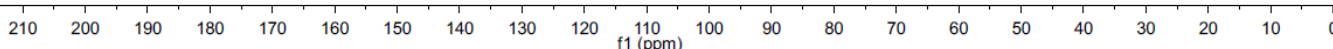




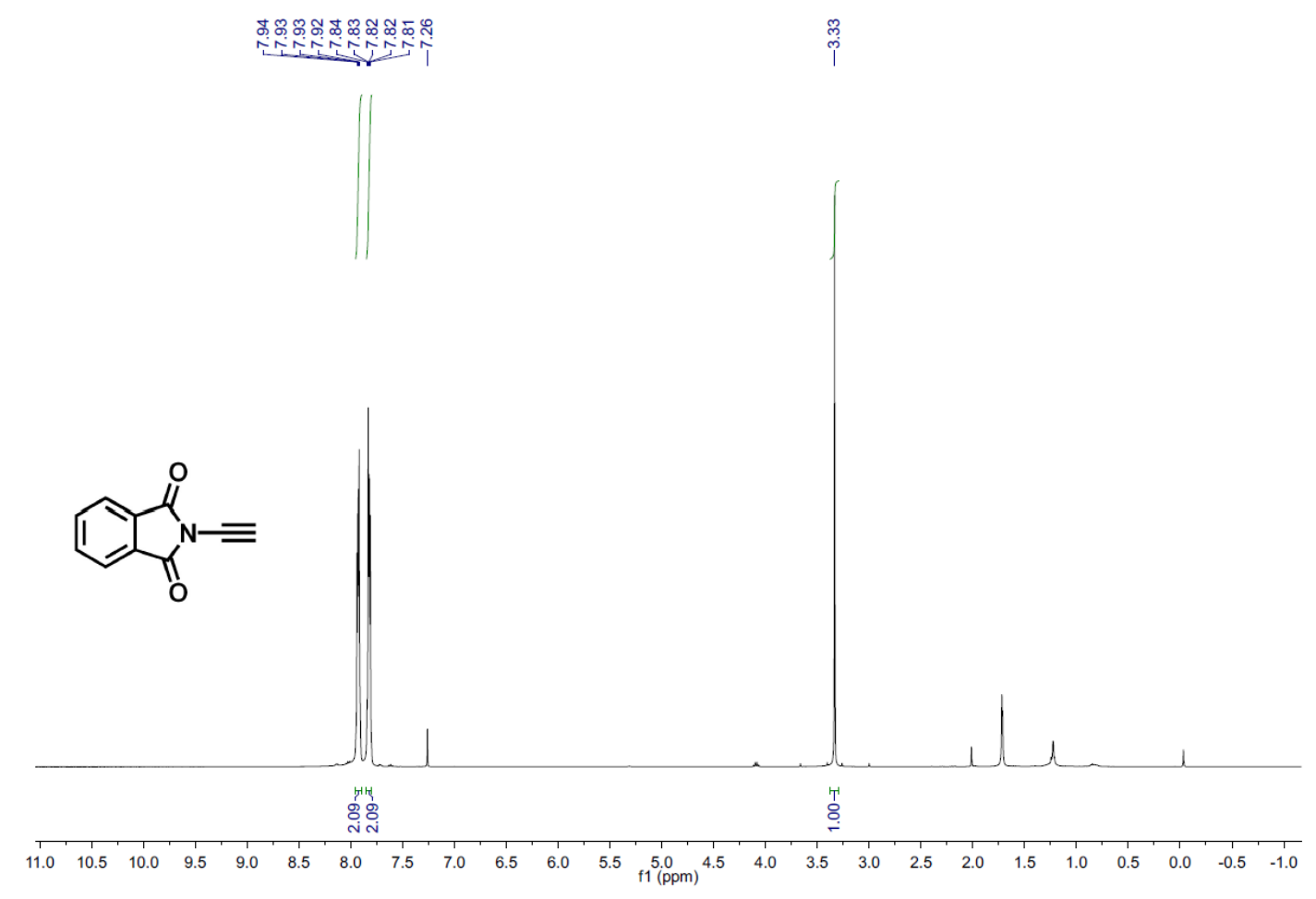

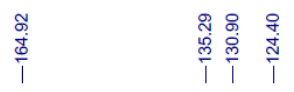

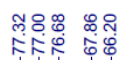
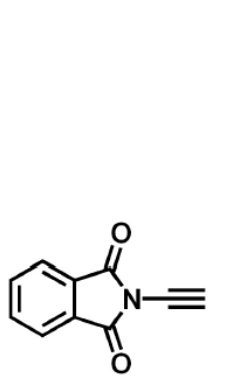

$\begin{array}{lllllllllllllllllllllllllllllll}210 & 200 & 190 & 180 & 170 & 160 & 150 & 140 & 130 & 120 & 110 & 100 & 90 & 80 & 70 & 60 & 50 & 40 & 30 & 20 & 10 & 0\end{array}$ 

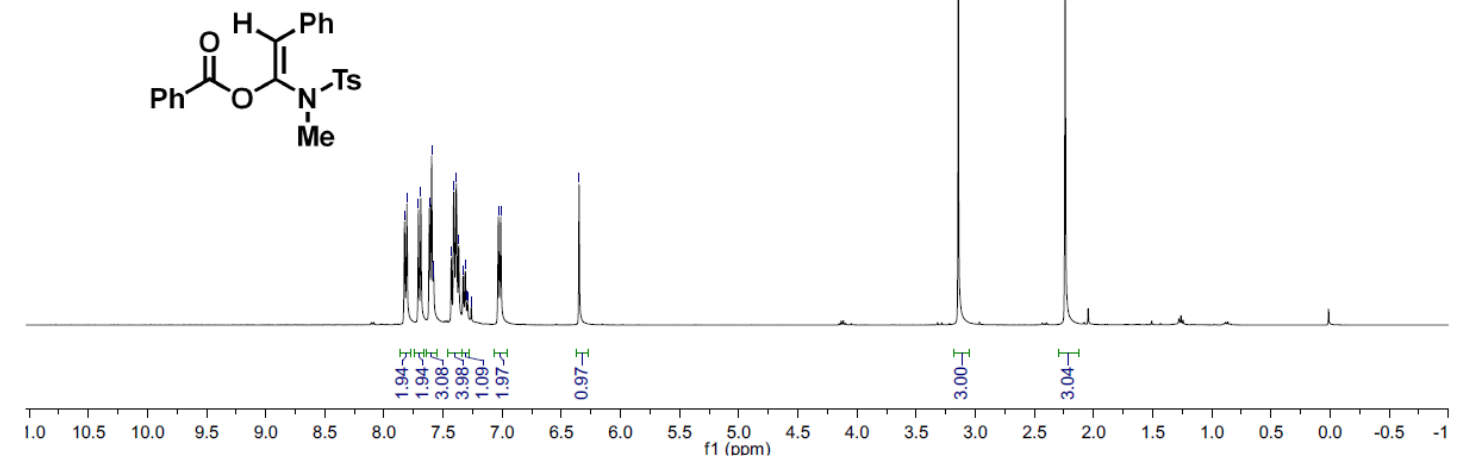

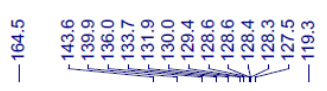

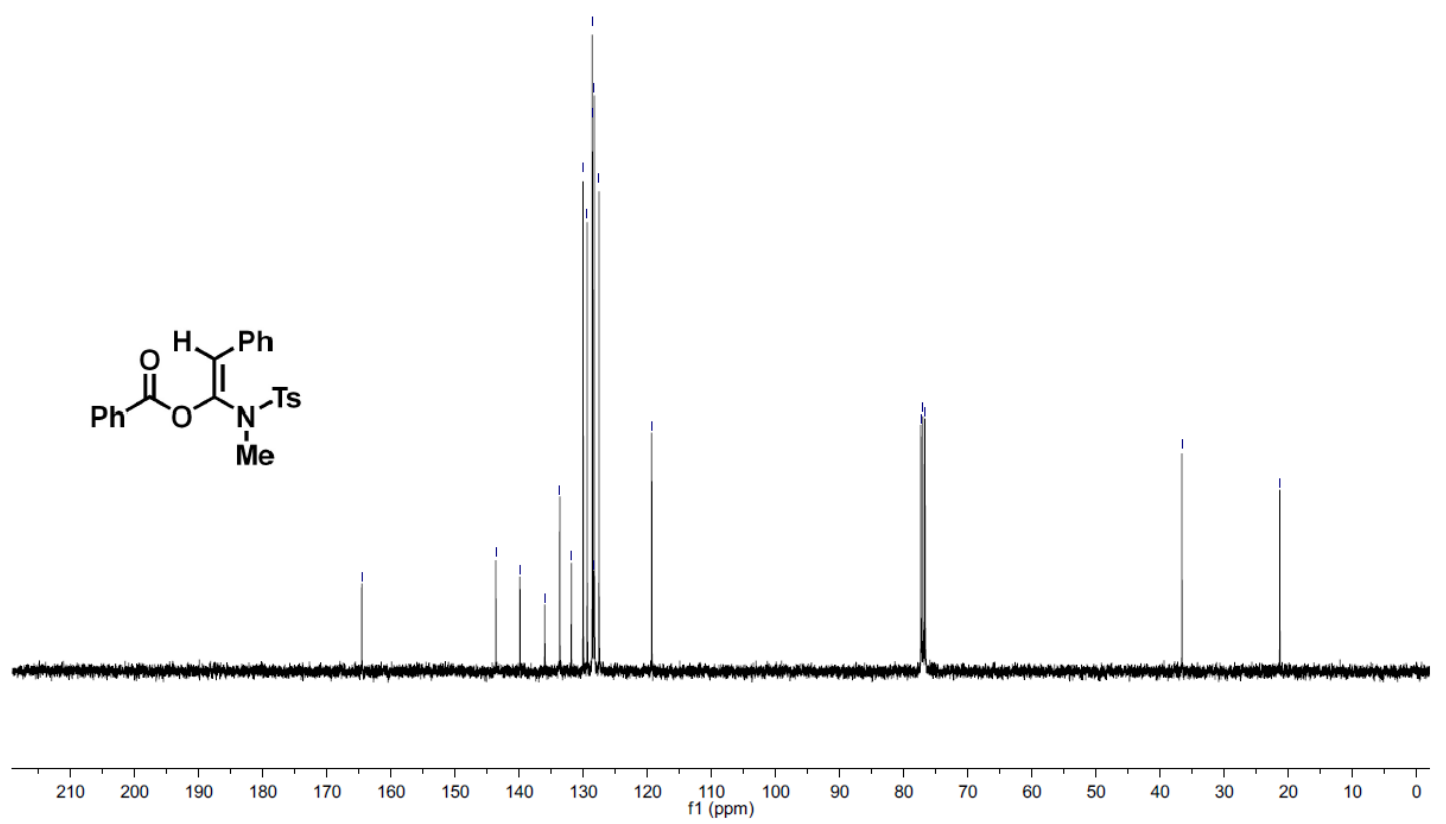


0
0

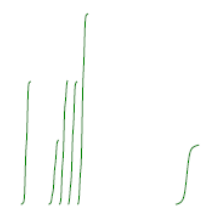

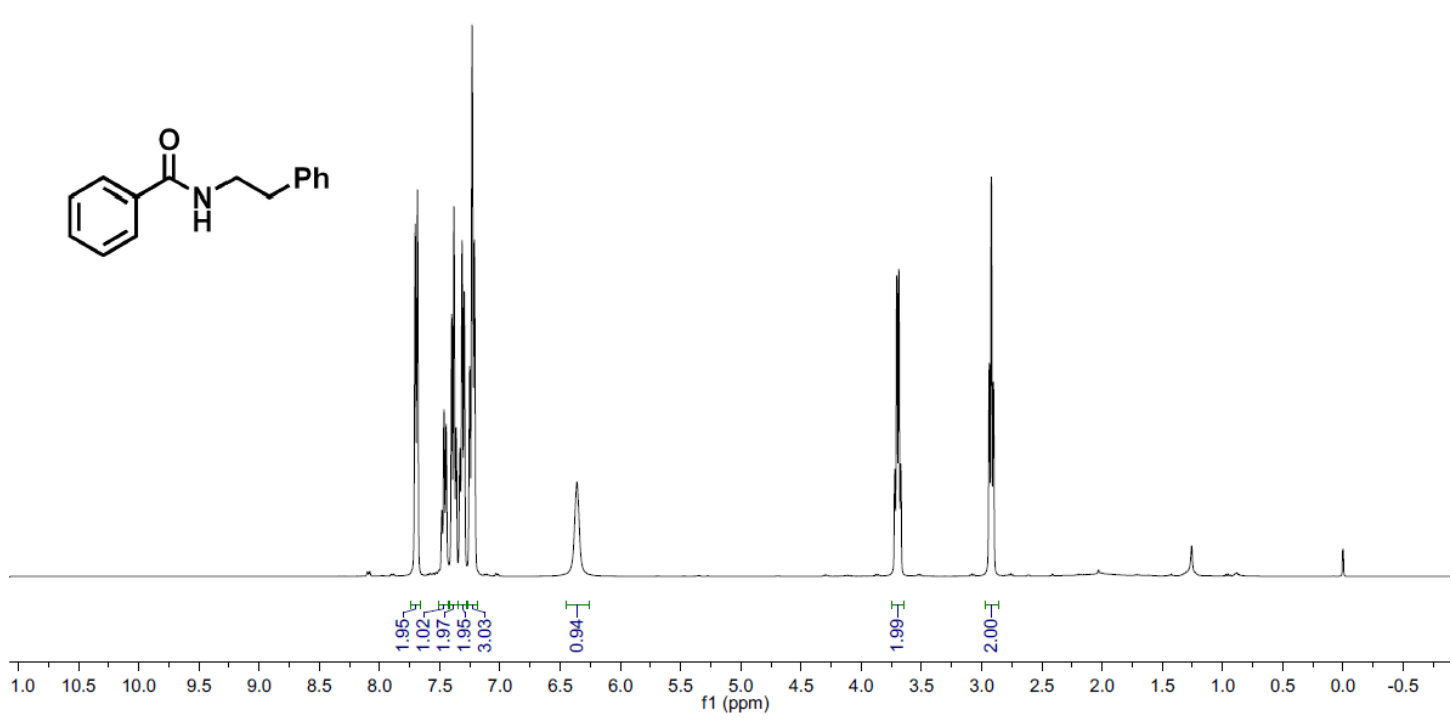

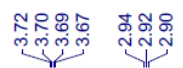

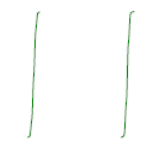

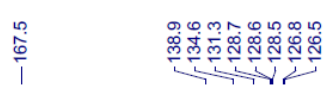

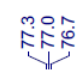

辛畩
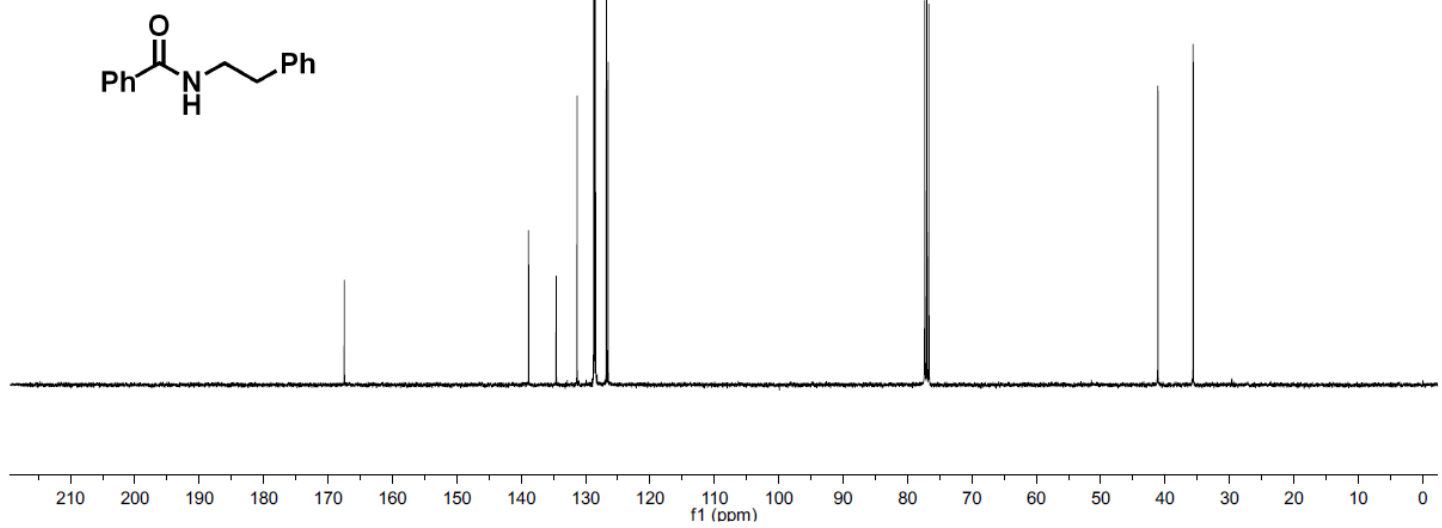

S54 


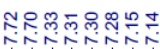

辛

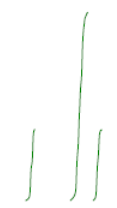

$\mathrm{Ph} \underbrace{\mathrm{O}}_{\substack{1 \\ \dot{M} e}}-\mathrm{Ts}$

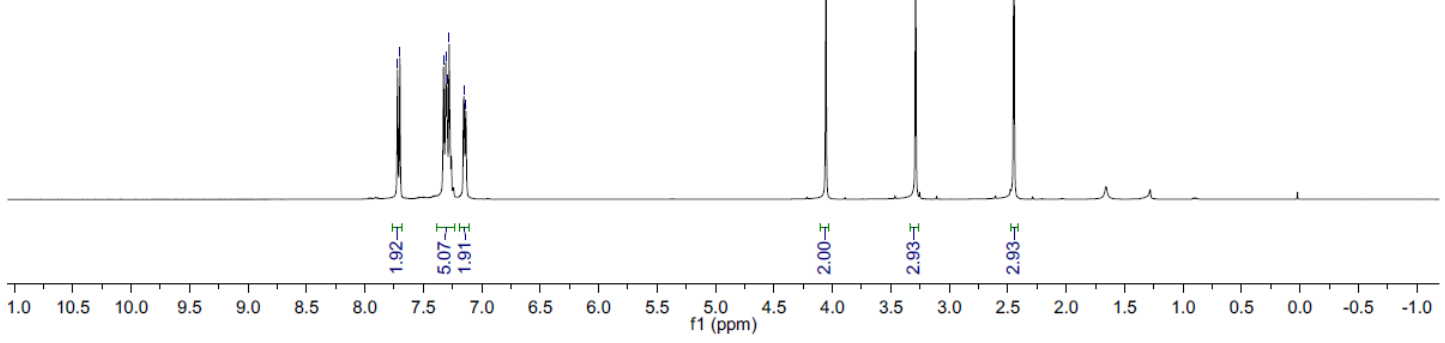
$\stackrel{?}{i}$

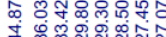

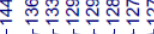
ํㅜㅇㅠ:
胥

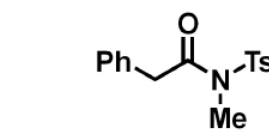

$N^{-T S}$
$\dot{M} e$

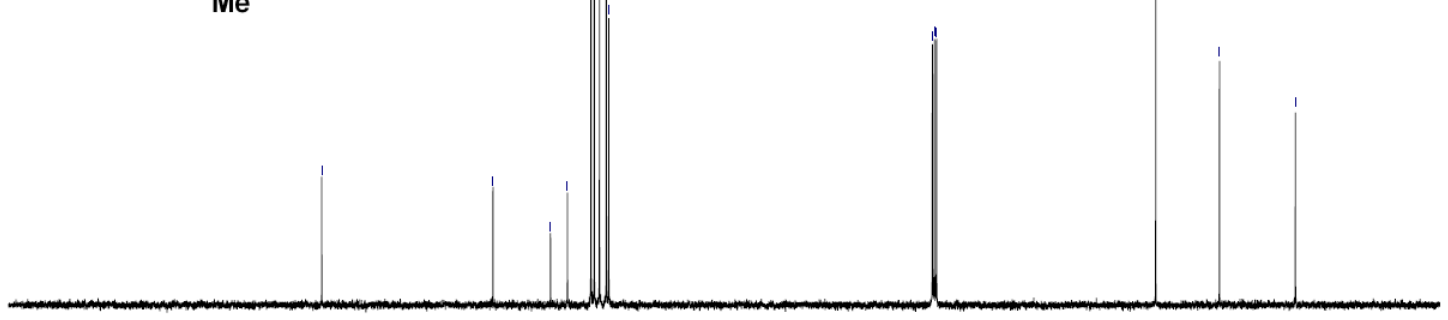

$\begin{array}{lllllllllllllllllllllll}210 & 200 & 190 & 180 & 170 & 160 & 150 & 140 & 130 & 120 & \begin{array}{c}110 \\ \mathrm{f} 1(\mathrm{ppm})\end{array} & 100 & 90 & 80 & 70 & 60 & 50 & 40 & 30 & 20 & 10 & 0\end{array}$ 


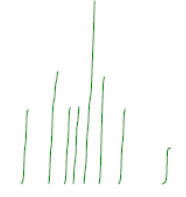

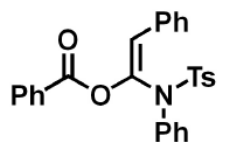

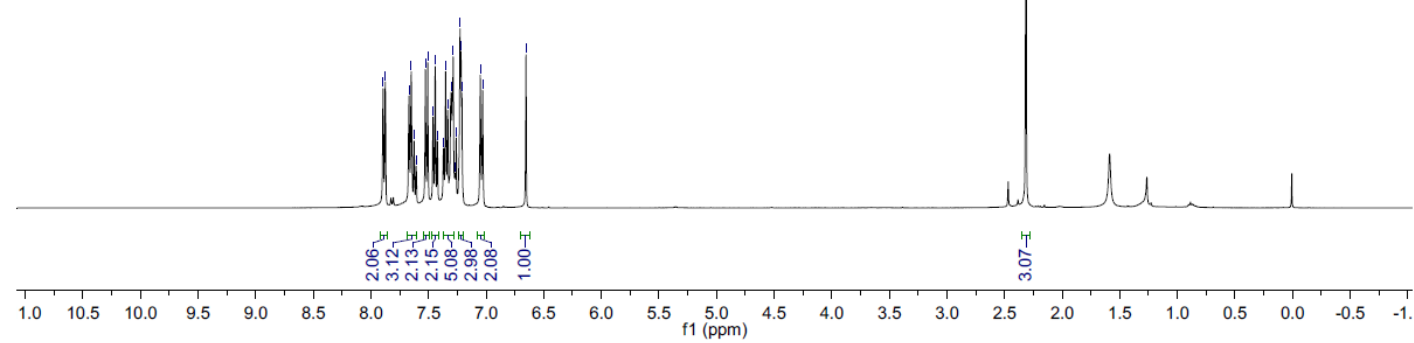

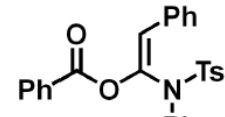

Ph

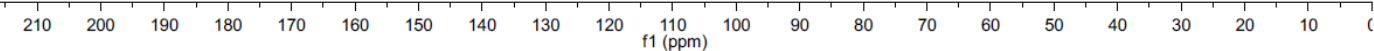


<smiles>O=C(OC(=C[PH-])c1ccccc1)N([AsH3-])Cc1ccccc1</smiles>

Bn

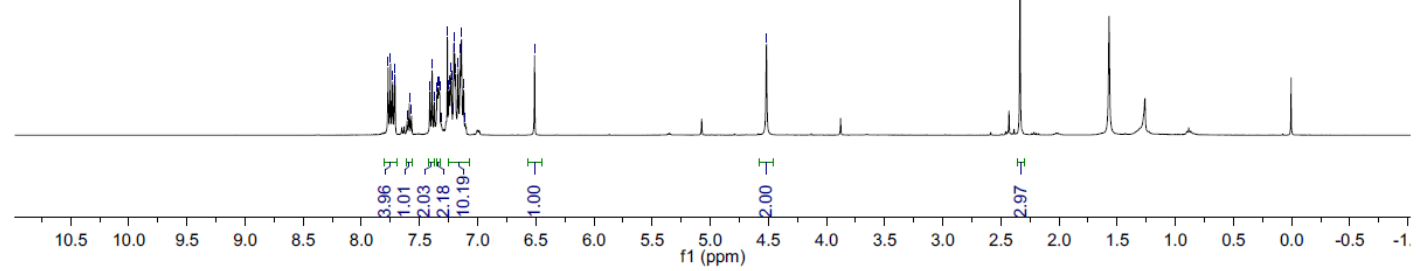<smiles></smiles>

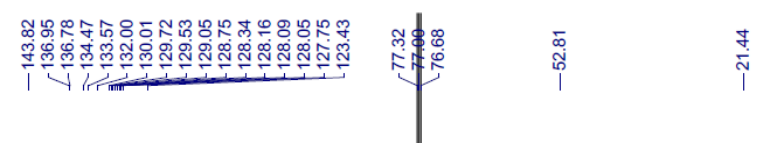

$\begin{array}{lllllllllllllllllllllll}210 & 200 & 190 & 180 & 170 & 160 & 150 & 140 & 130 & 120 & 110 & 100 & 90 & 80 & 70 & 60 & 50 & 40 & 30 & 20 & 10 & 0 & -10\end{array}$ 
密

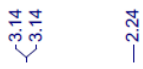
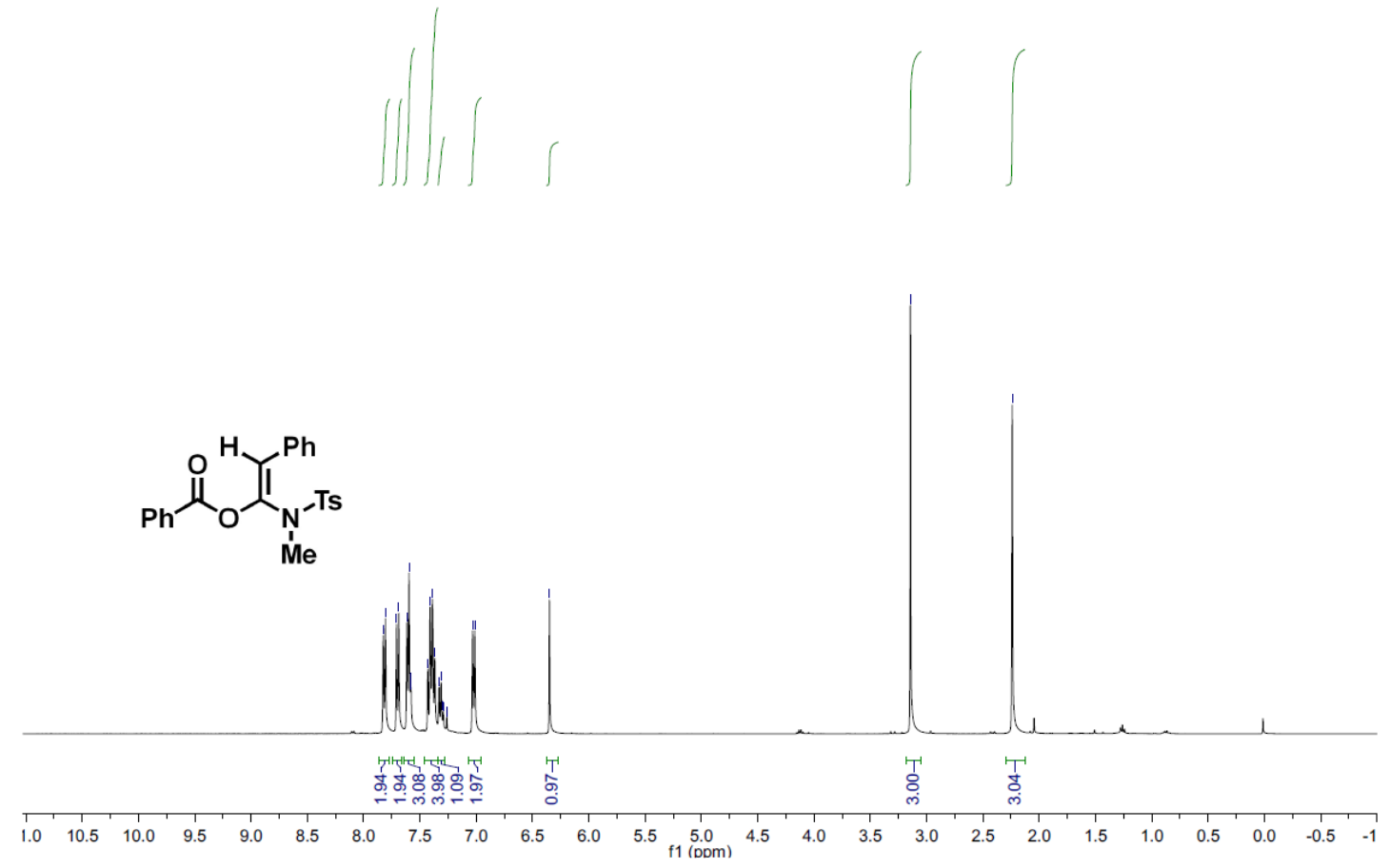

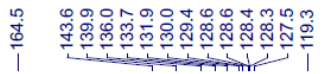

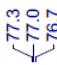

$\stackrel{\substack{0 \\ i}}{i}$
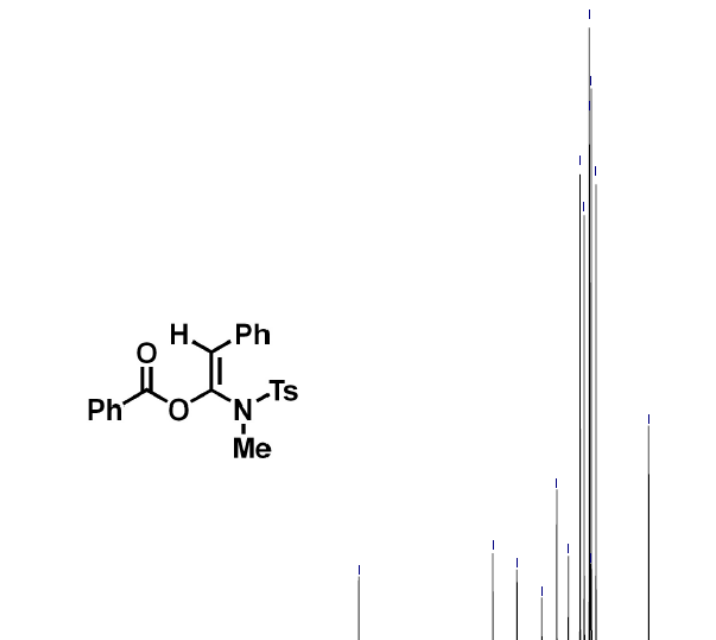

$\begin{array}{llllllllllllllllllllllll}1 & 1 & 200 & 190 & 180 & 170 & 160 & 150 & 140 & 130 & 120 & \underset{f 1}{110} & 100 & 90 & 80 & 70 & 60 & 50 & 40 & 30 & 20 & 10 & 0\end{array}$ 


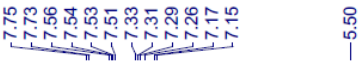

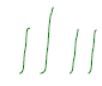

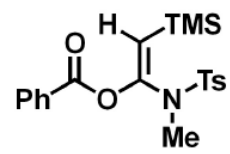

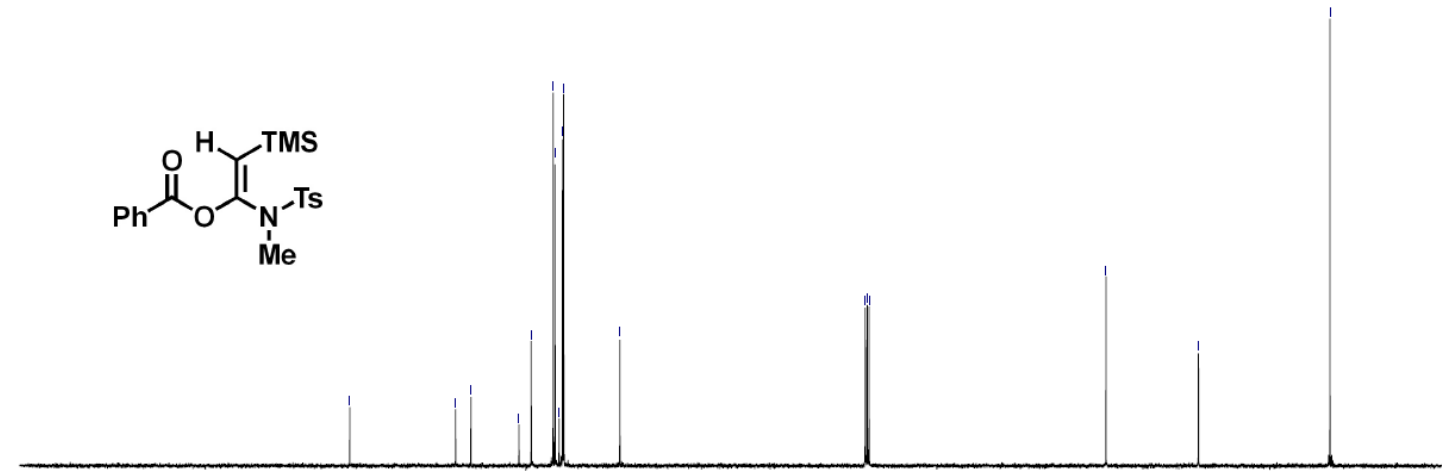

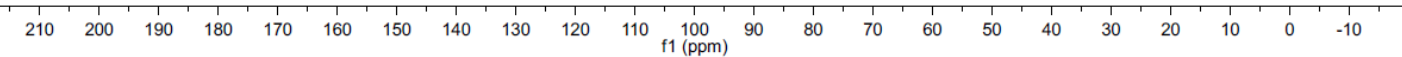




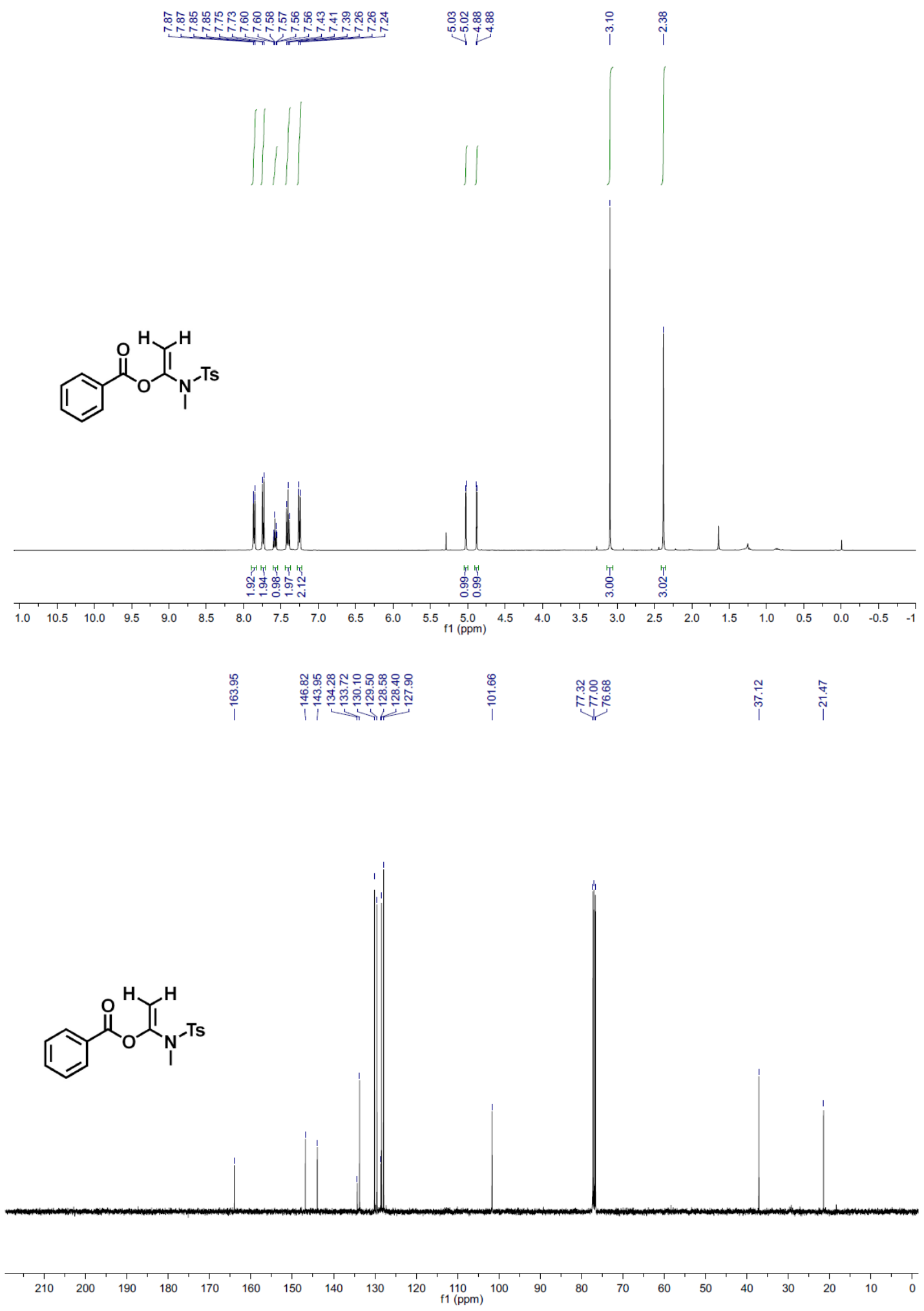




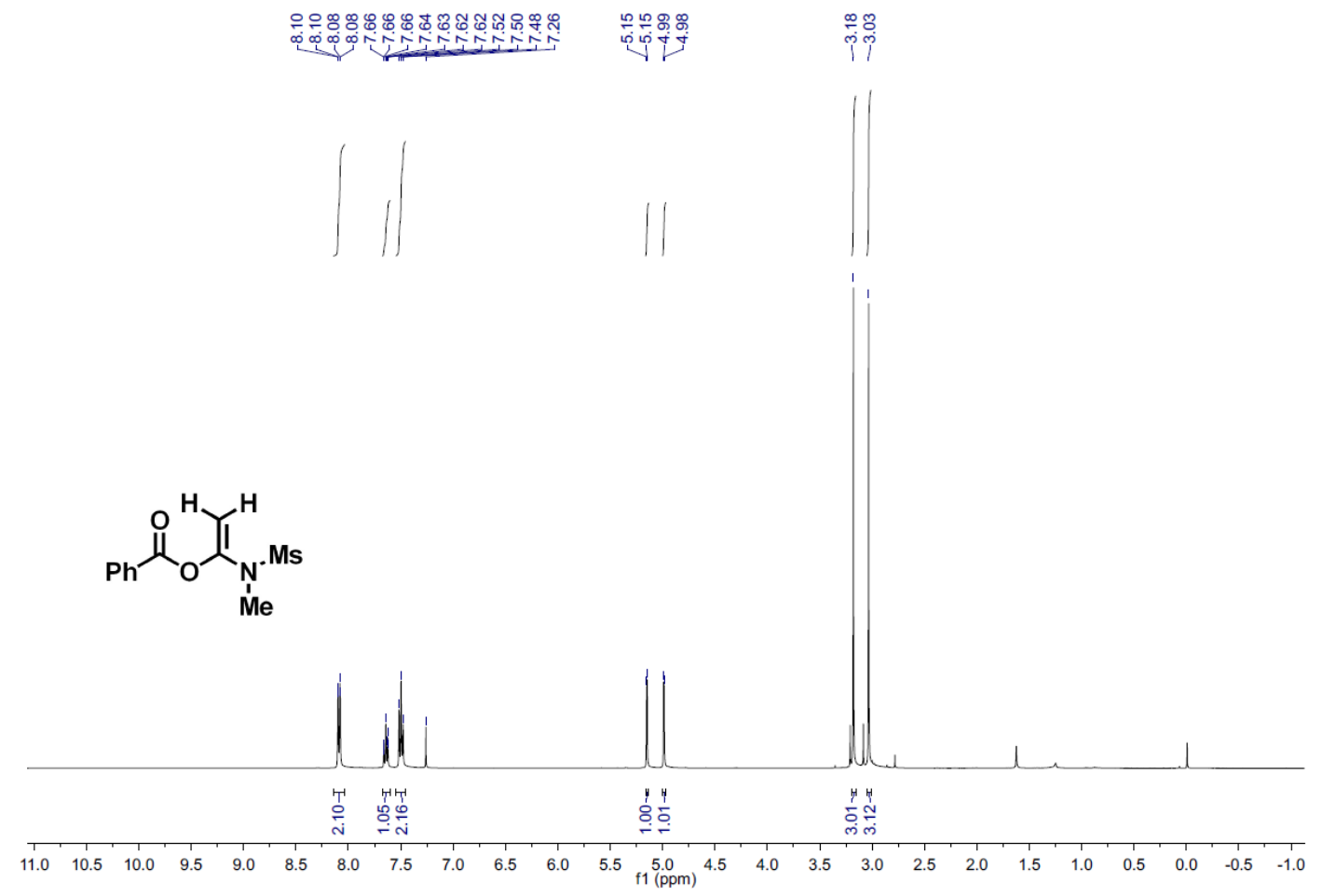

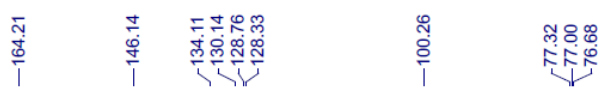
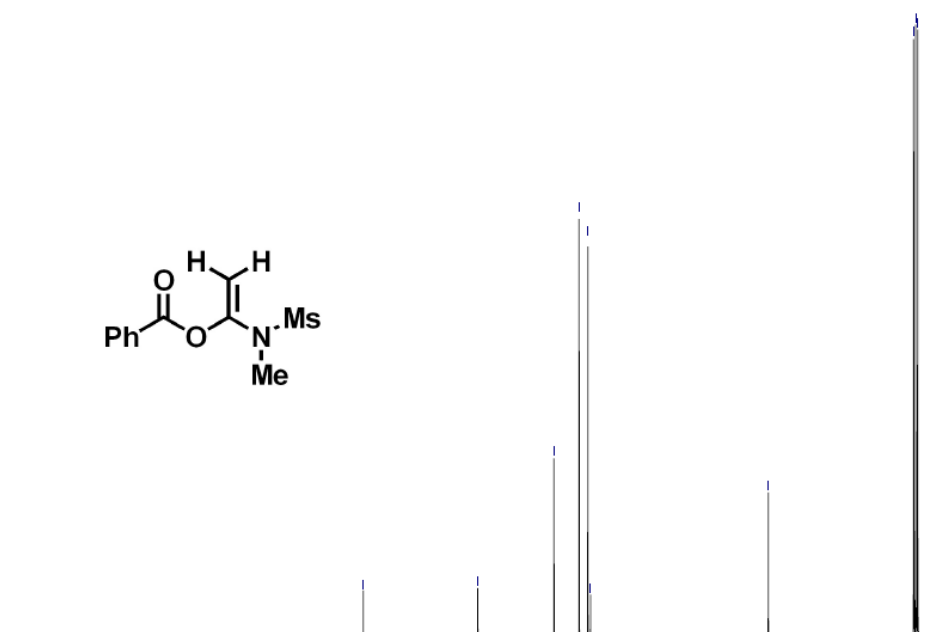

$\begin{array}{llllllllllllllllllllllllllllllllll} & 210 & 200 & 190 & 180 & 170 & 160 & 150 & 140 & 130 & 120 & 110 & 100 & 90 & 80 & 70 & 60 & 50 & 40 & 30 & 20 & 10 & 0\end{array}$ 


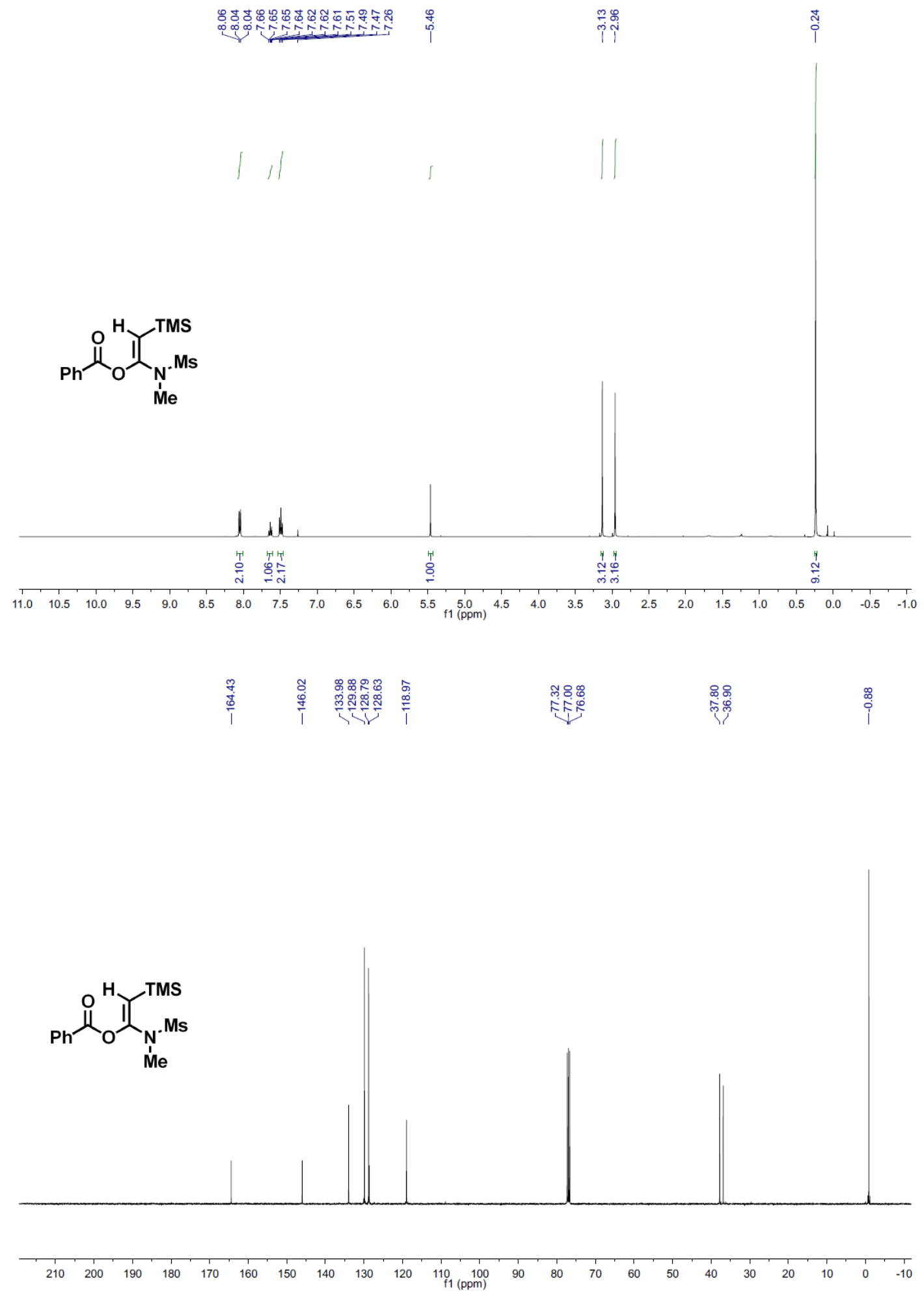




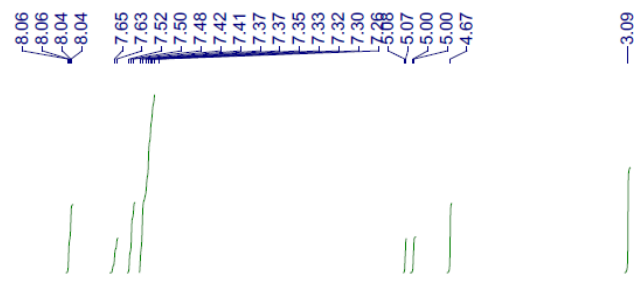

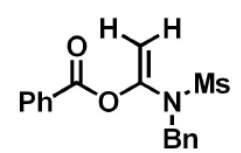
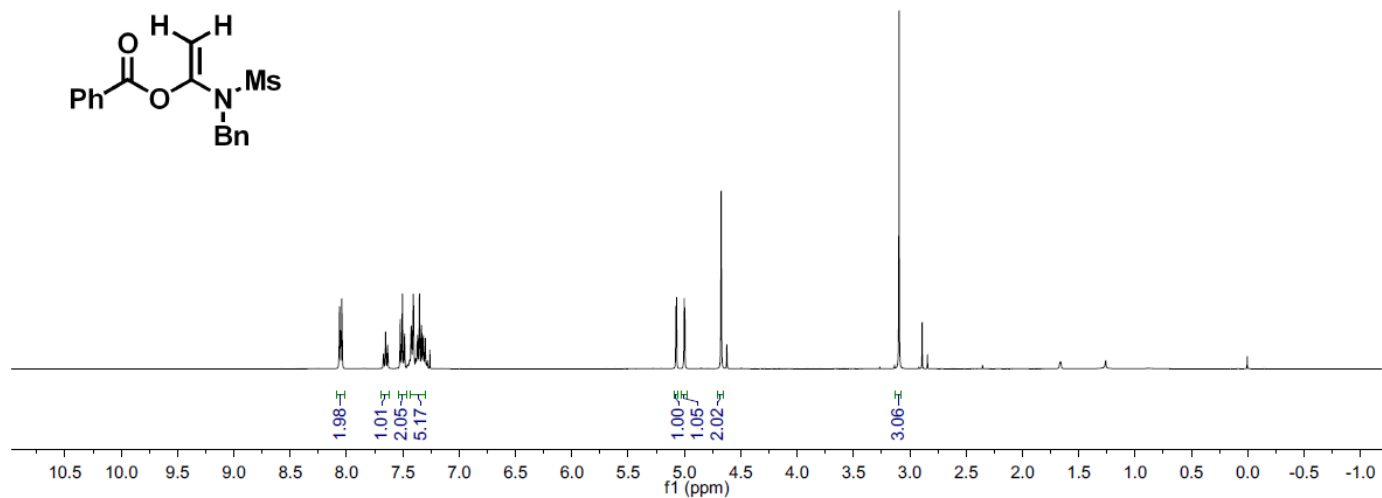

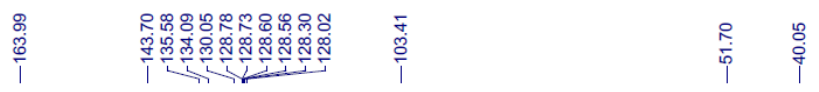

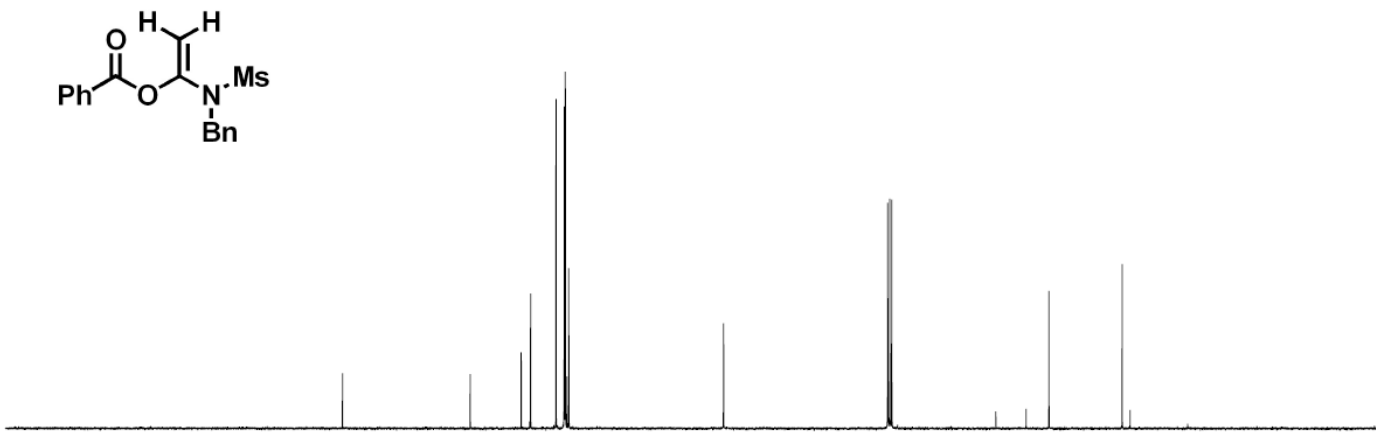

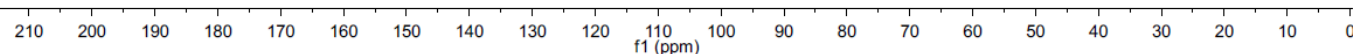




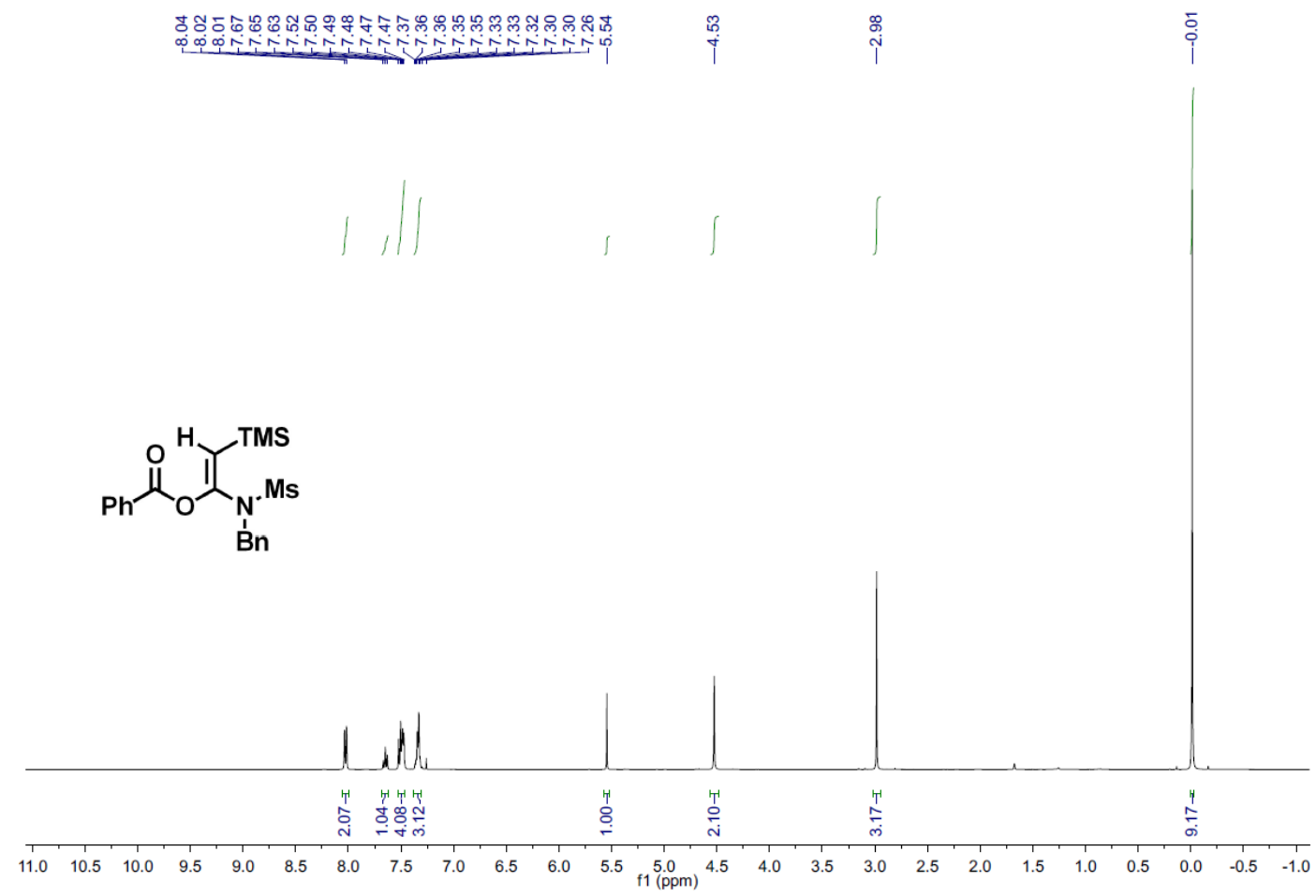

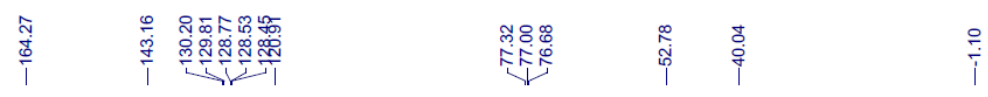

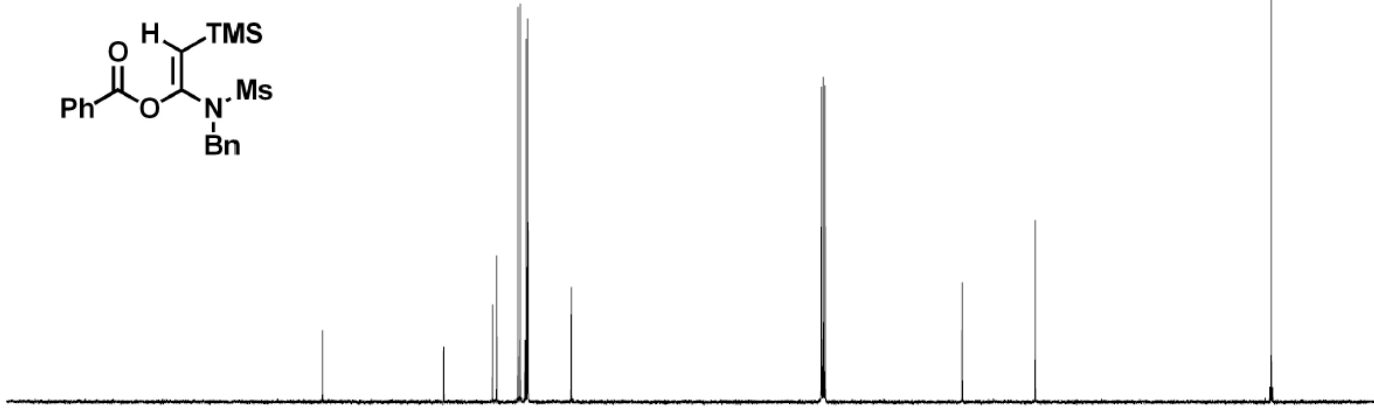

$\begin{array}{lllllllllllllllllllllllllllll}210 & 200 & 190 & 180 & 170 & 160 & 150 & 140 & 130 & 120 & 110 & 100 & 90 & 10 & 70 & 60 & 50 & 40 & 30 & 20 & 10 & 0 & -10\end{array}$ 


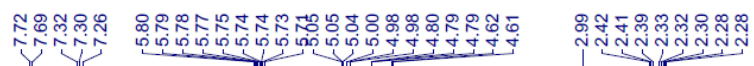
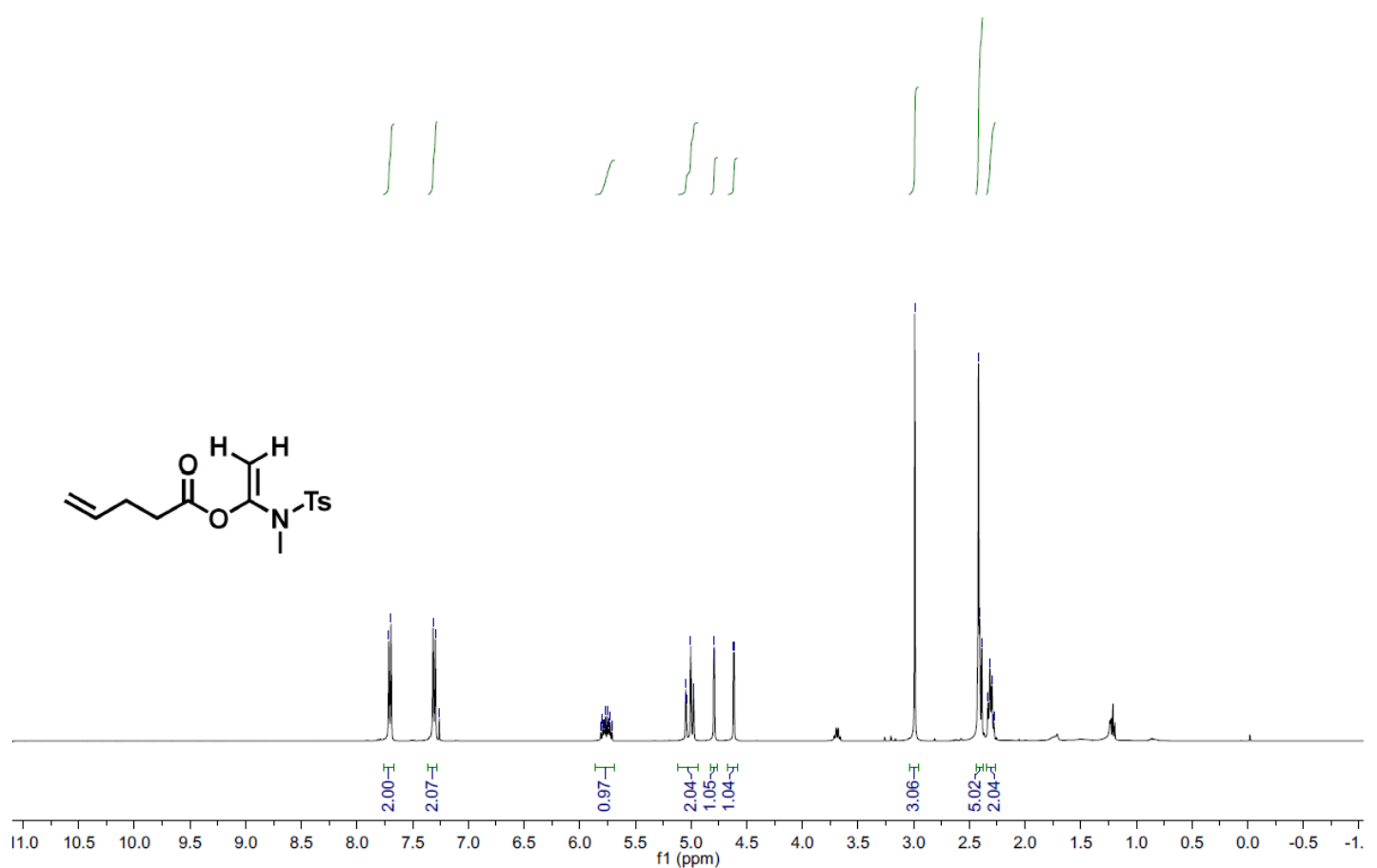

\begin{tabular}{|c|c|c|c|c|c|c|}
\hline 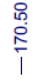 & 站尃 & 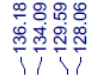 & 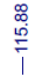 & i̊ & 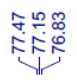 & 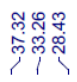 \\
\hline
\end{tabular}

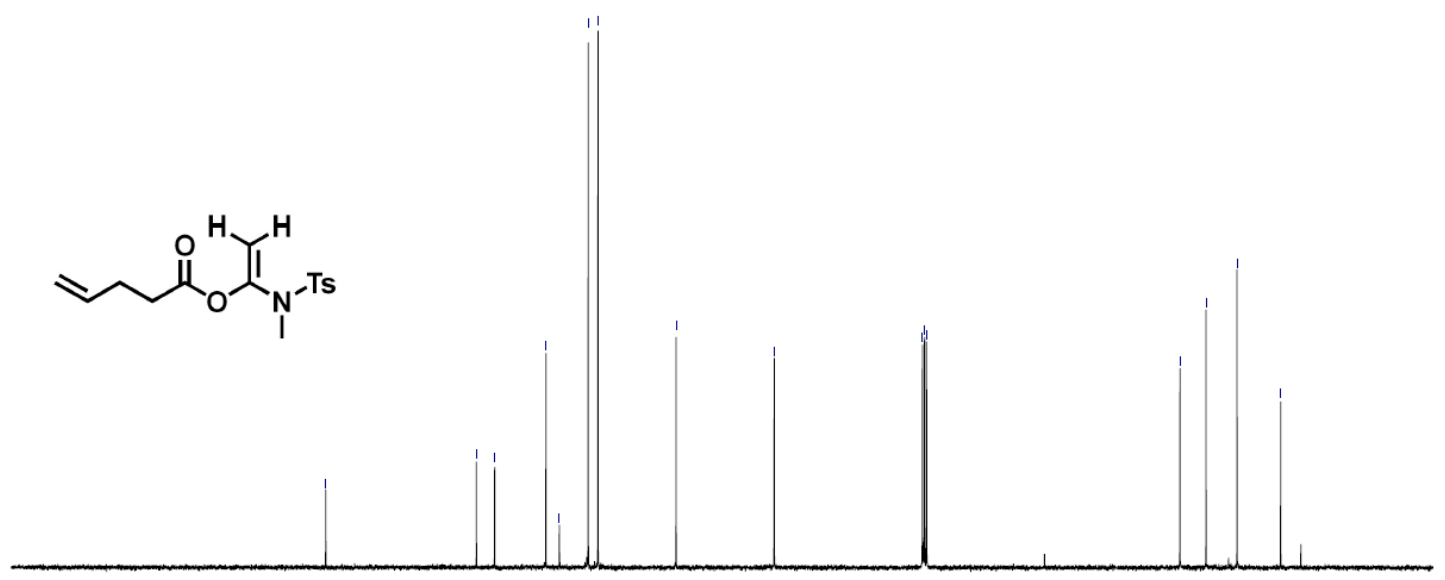

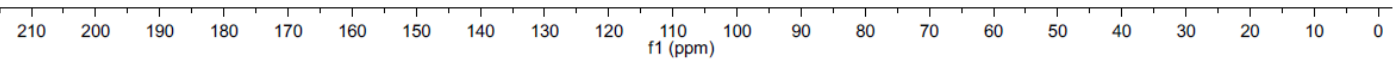




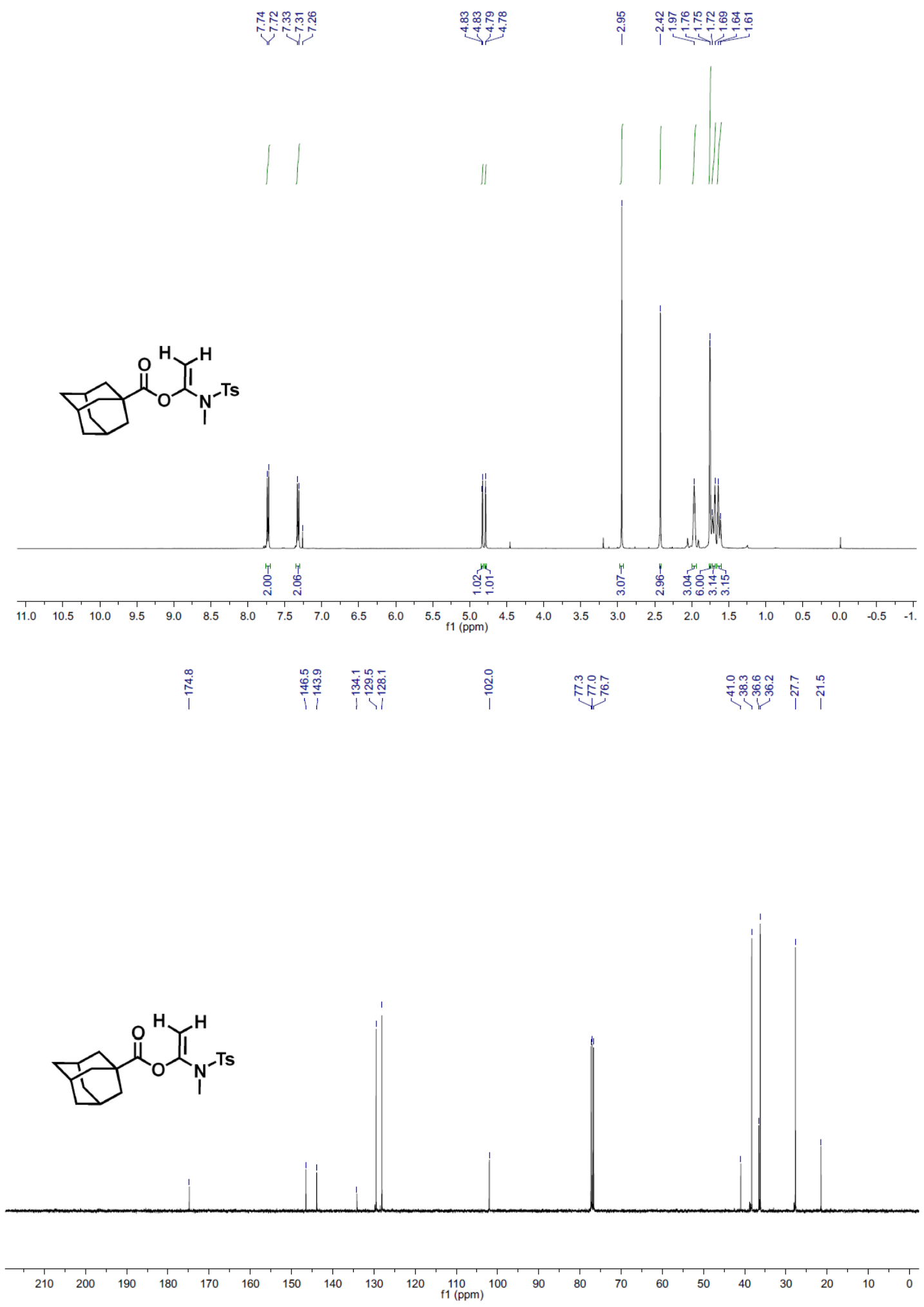



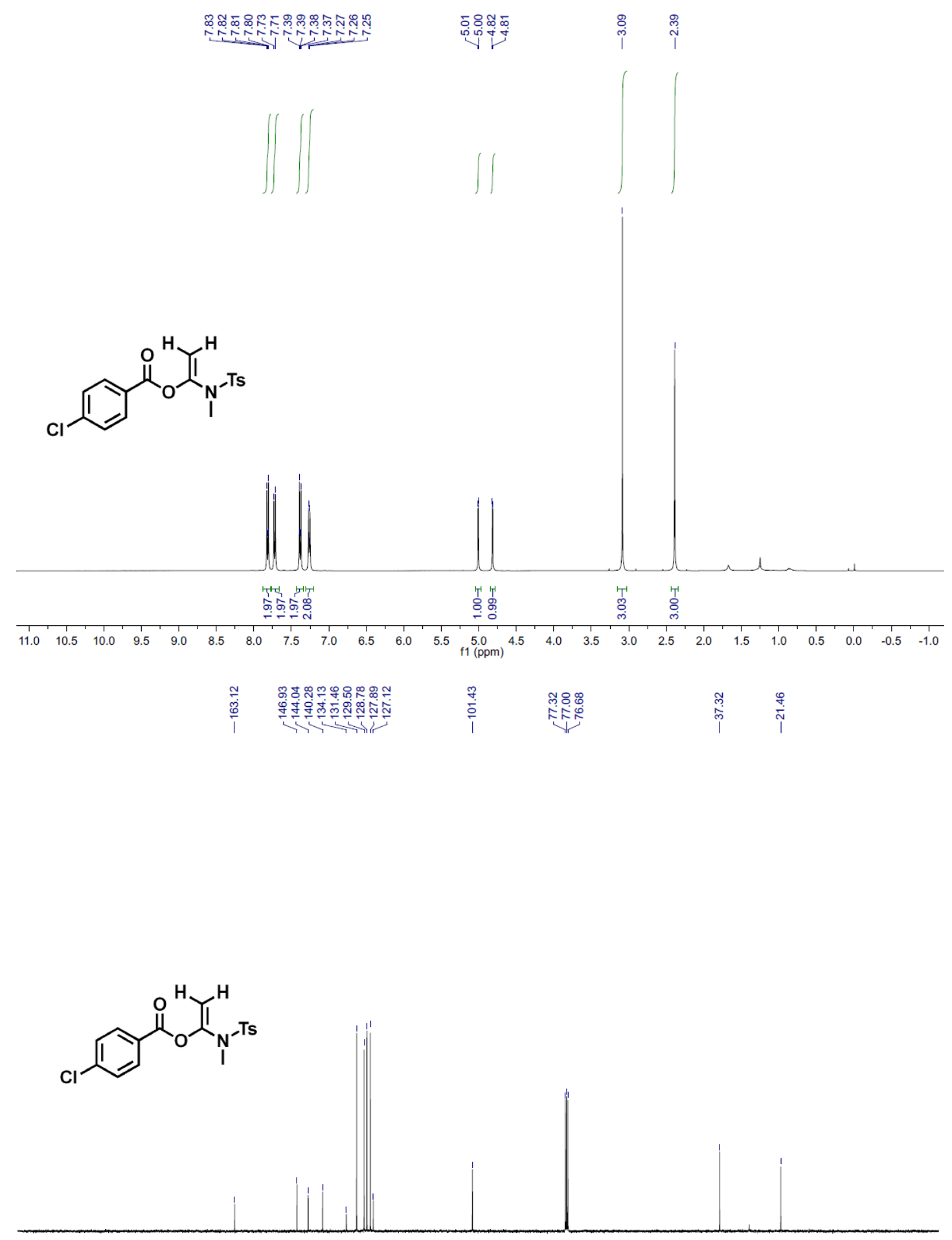

$\begin{array}{llllllllllllllllllllllllllll} & 1 \\ 210 & 200 & 190 & 180 & 170 & 160 & 150 & 140 & 130 & 120 & 110 & 100 & 90 & 80 & 70 & 60 & 50 & 40 & 30 & 20 & 10 & 0 & -10\end{array}$ 

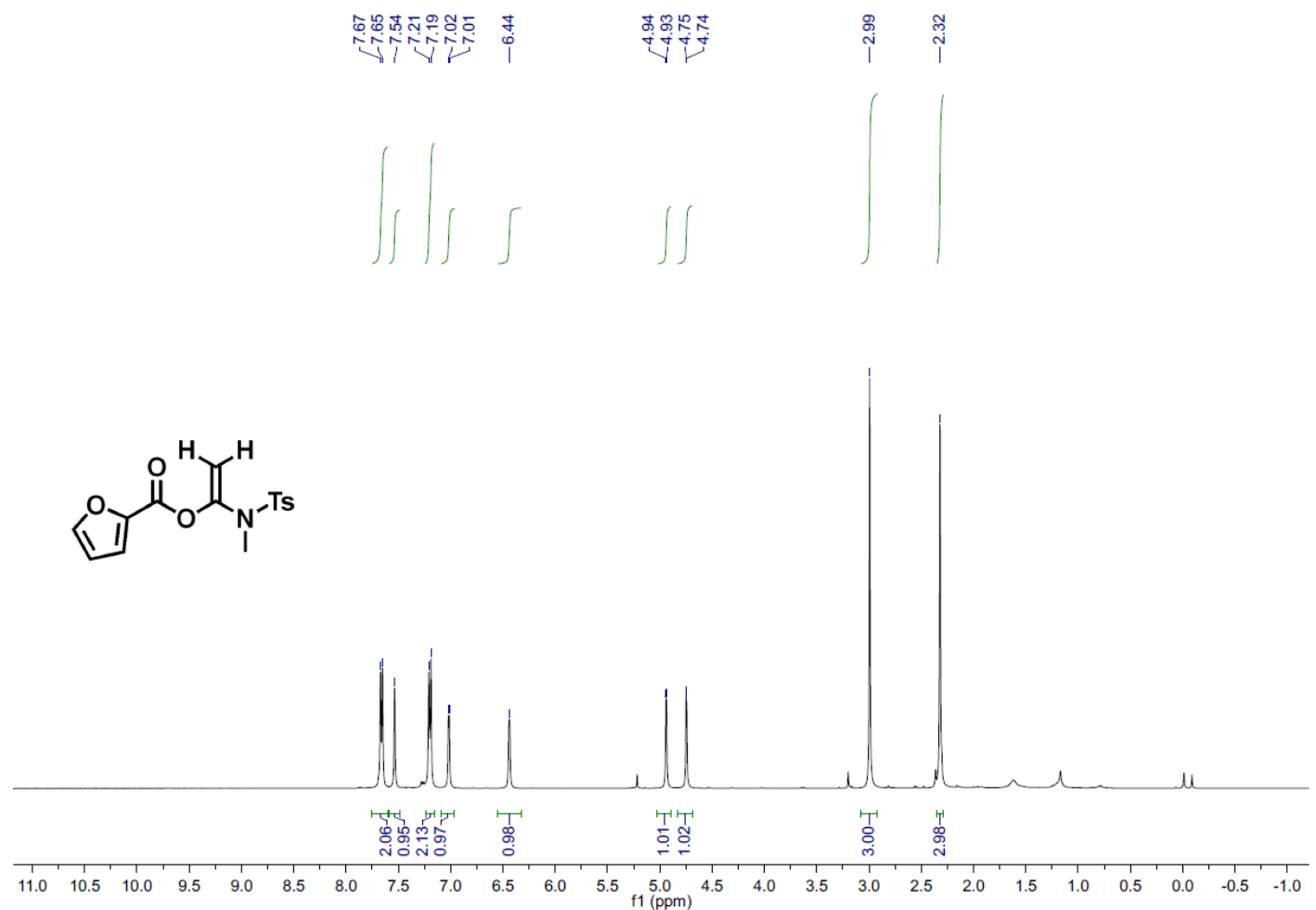

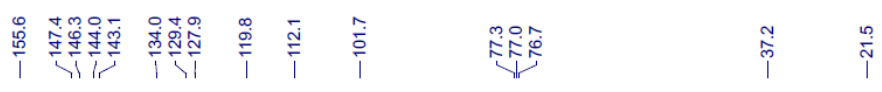

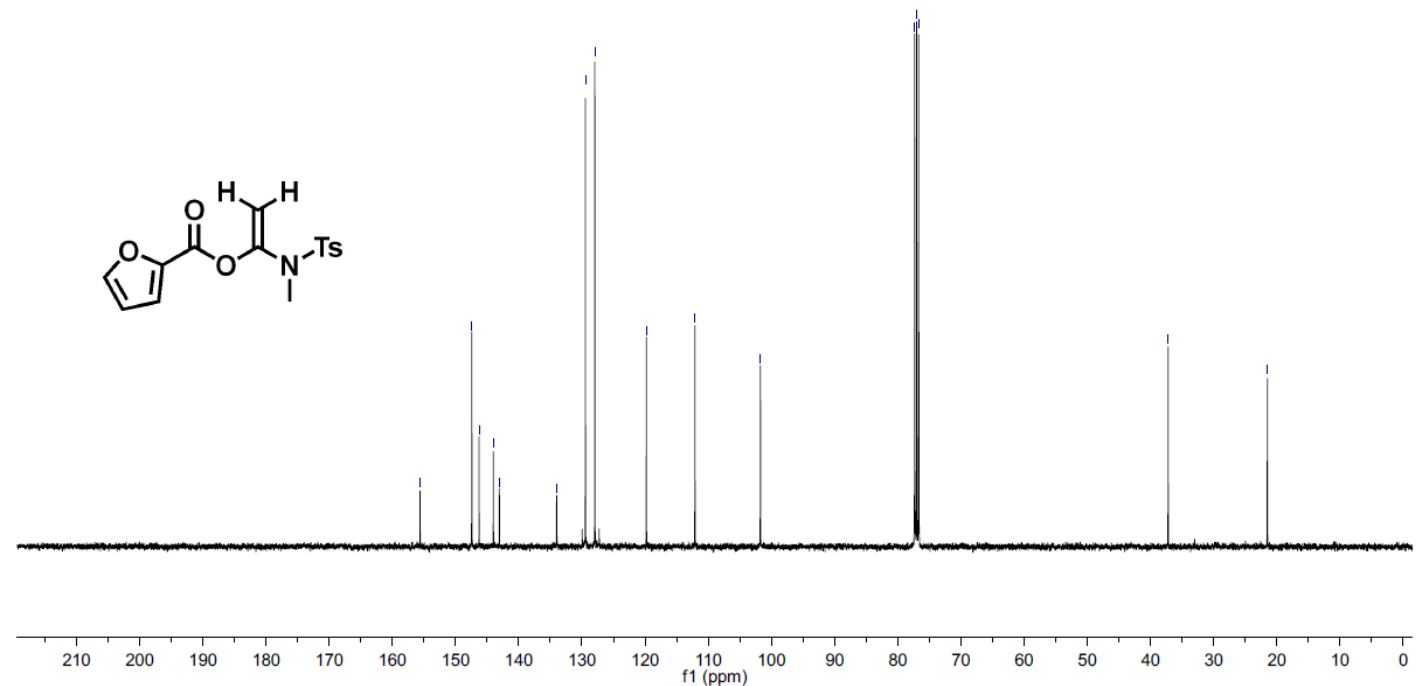



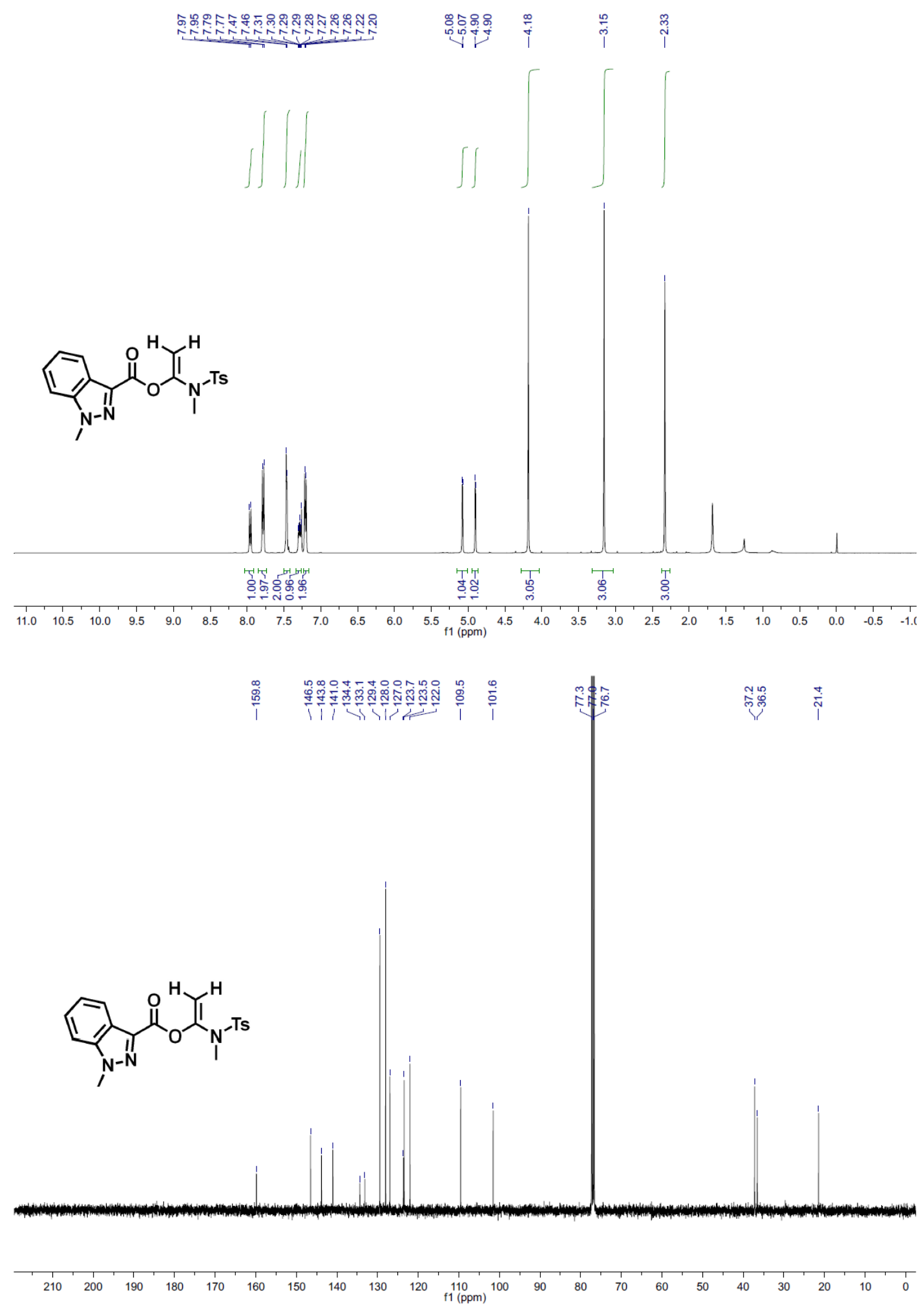


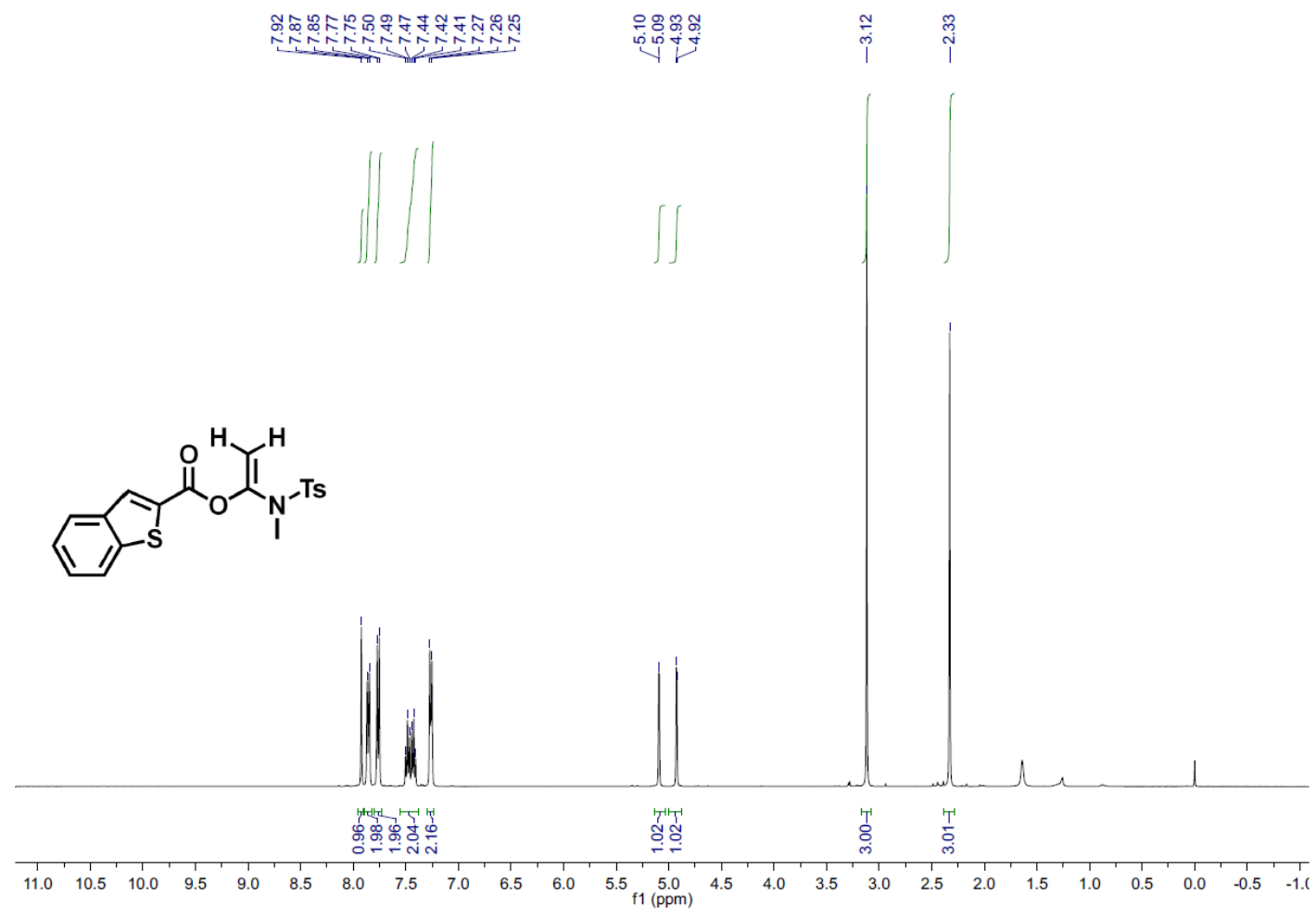

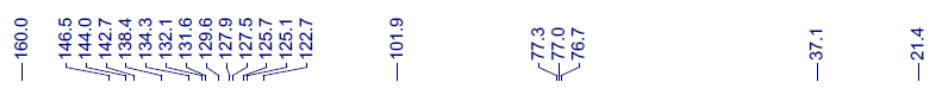

-

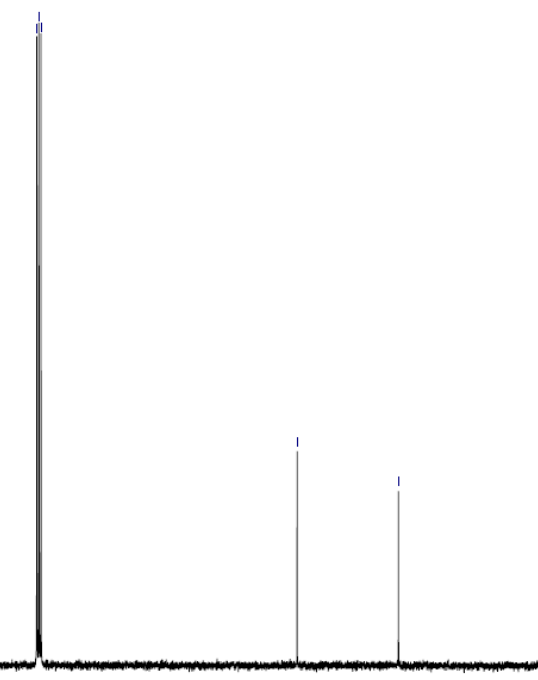

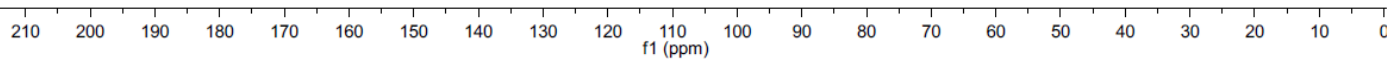



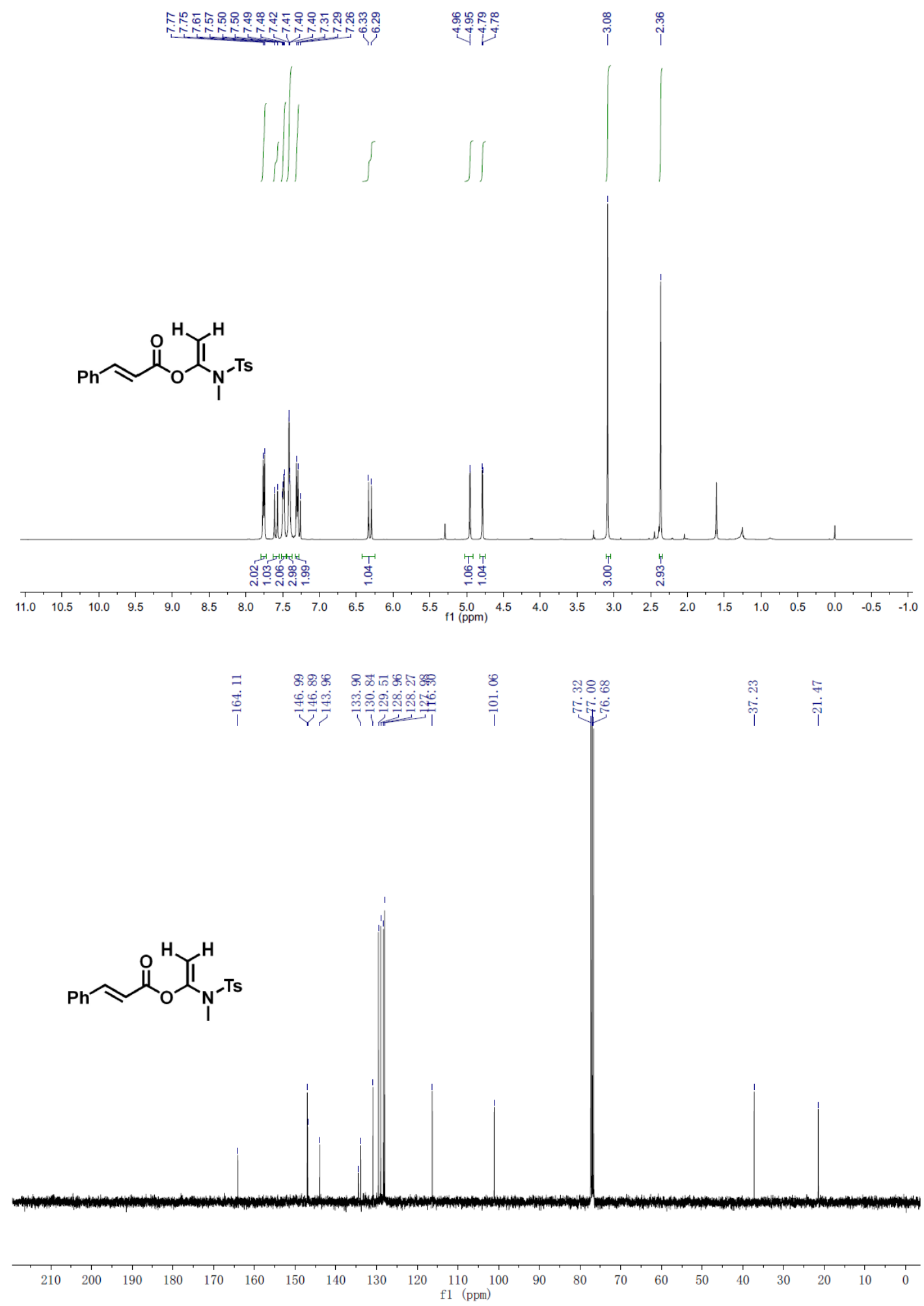


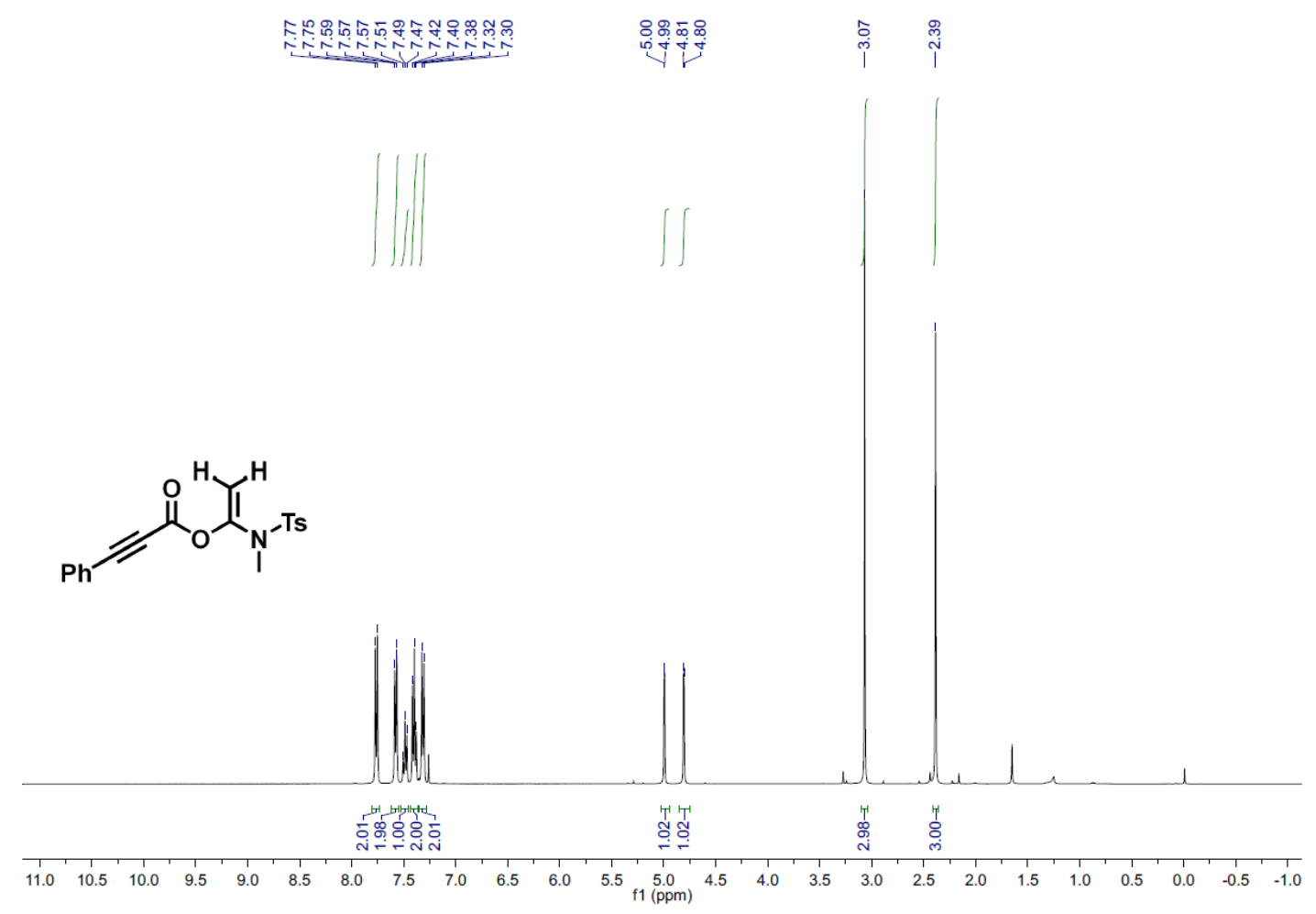

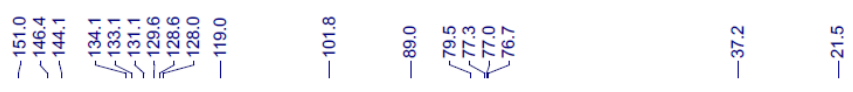

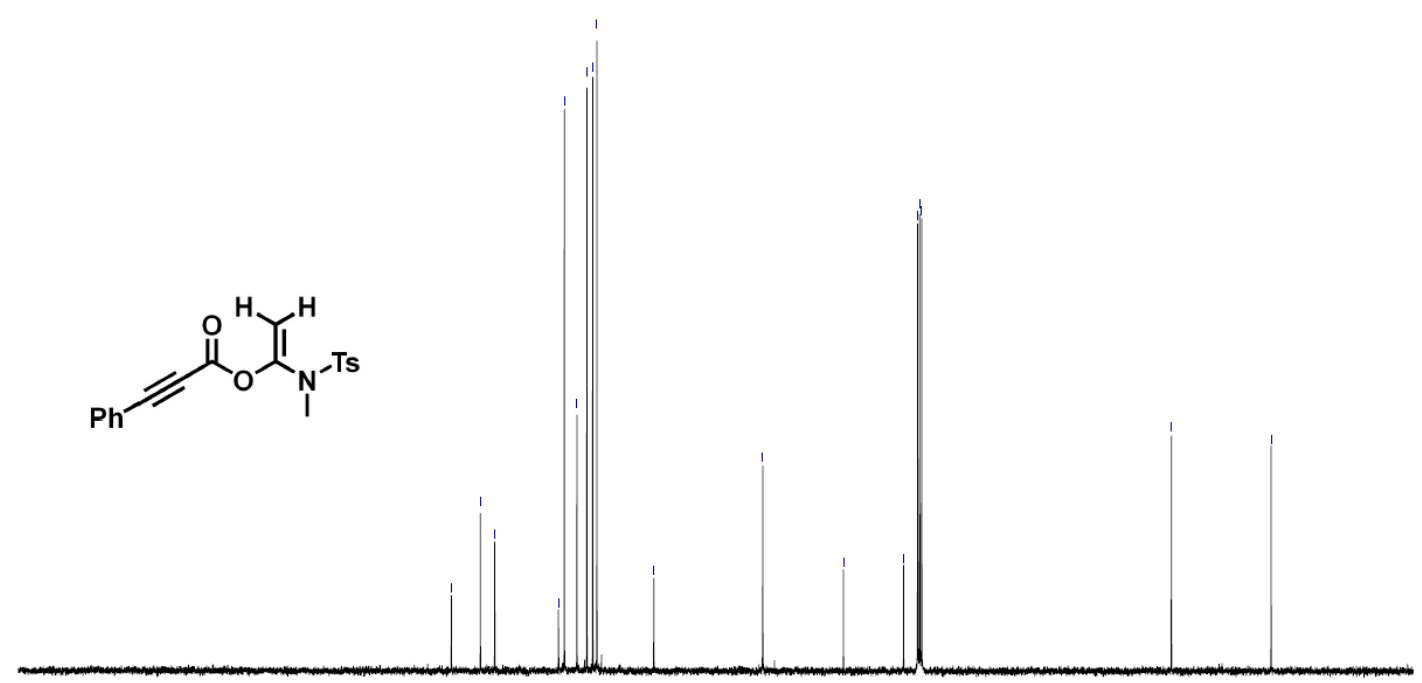

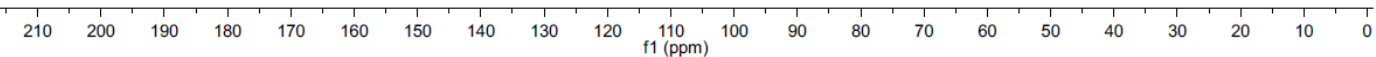



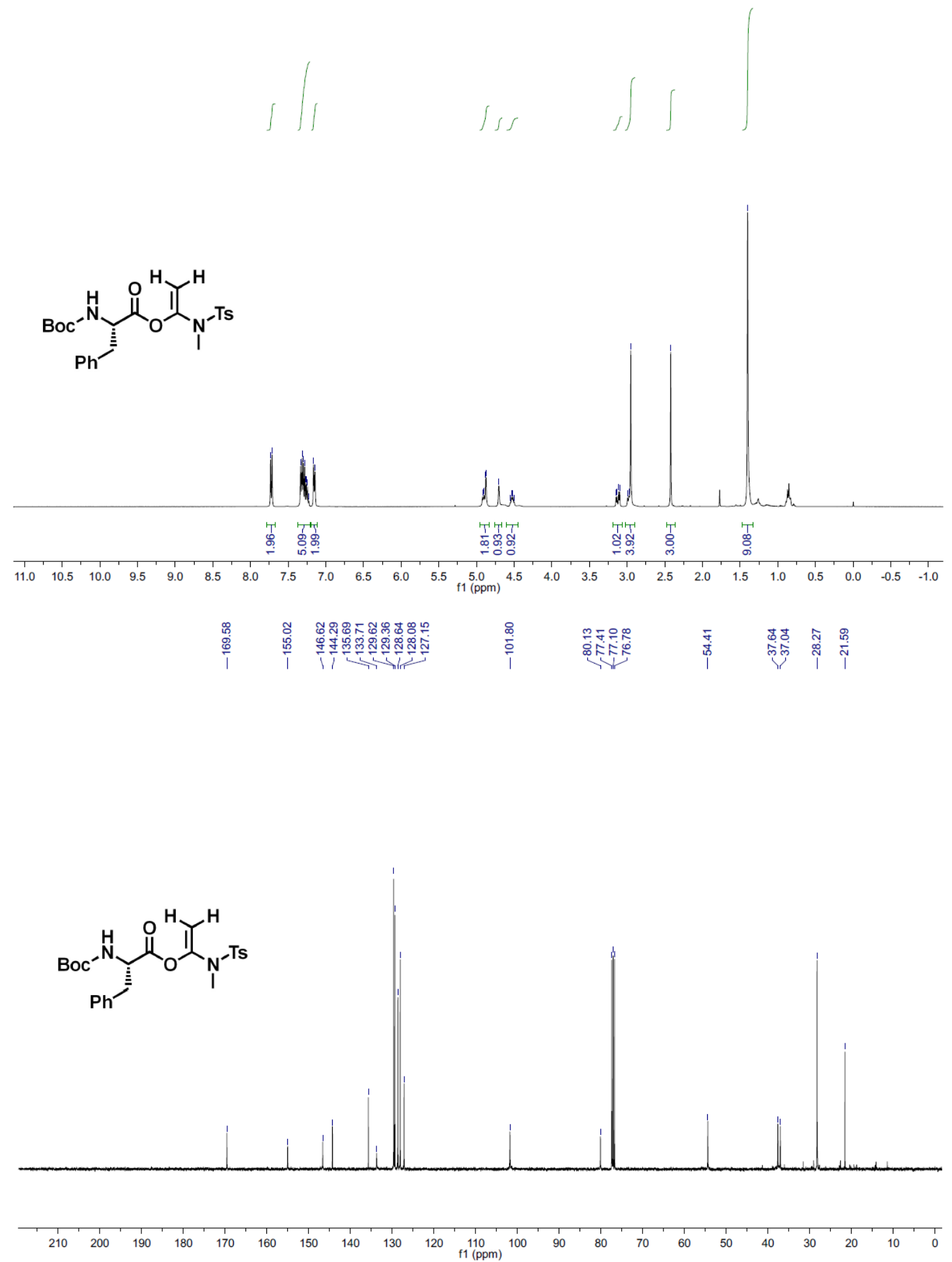


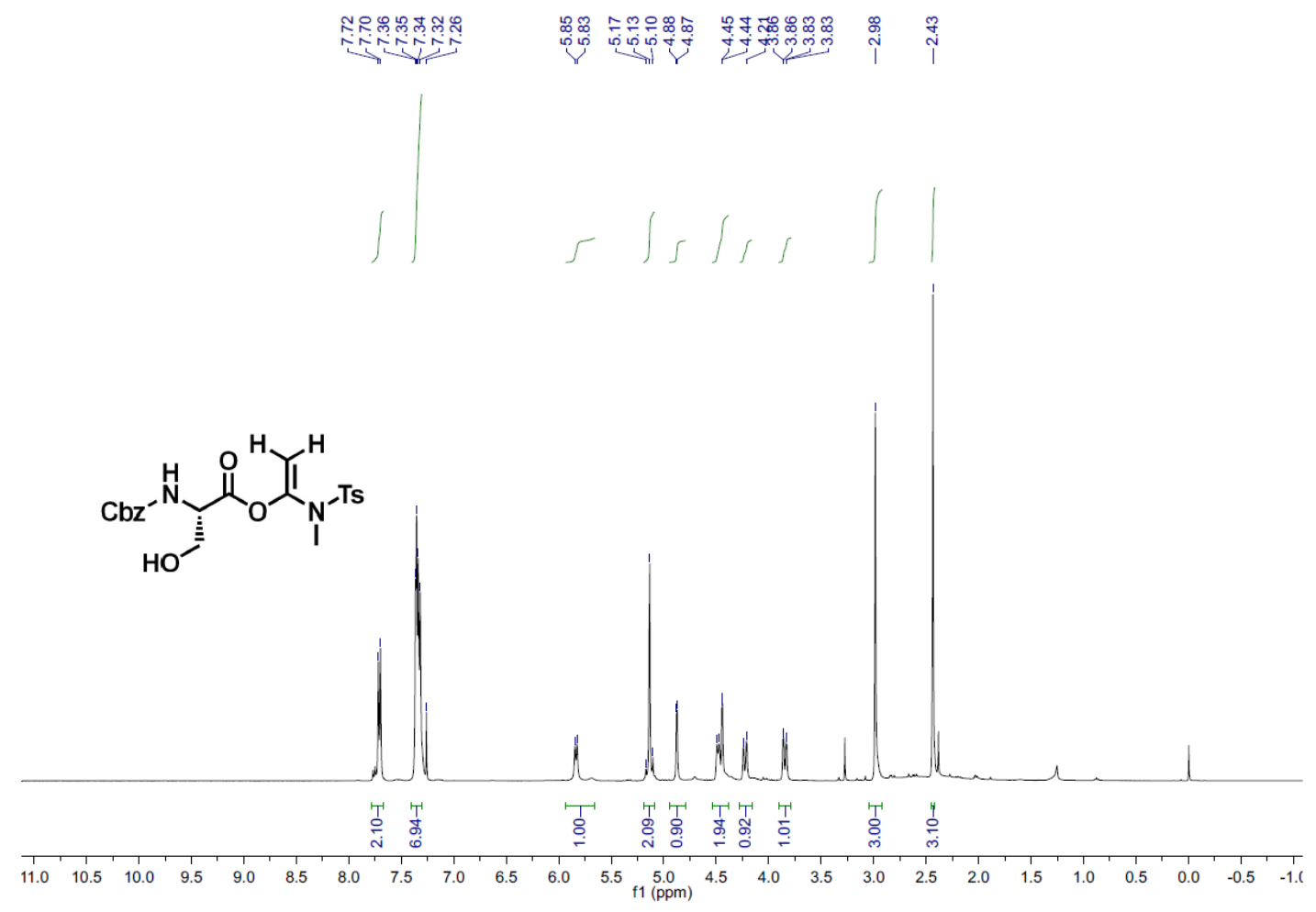

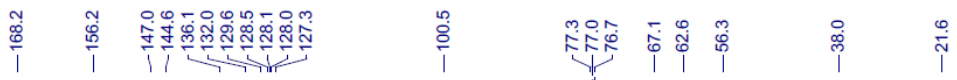

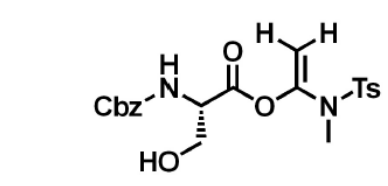

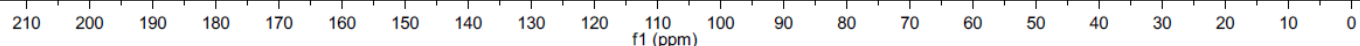




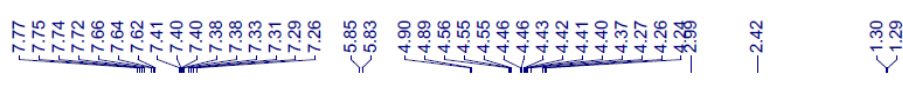

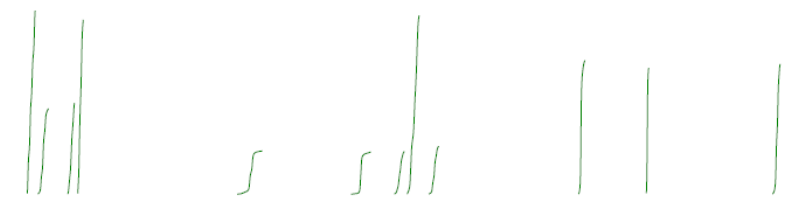

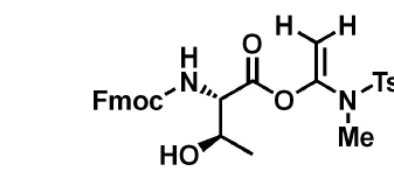

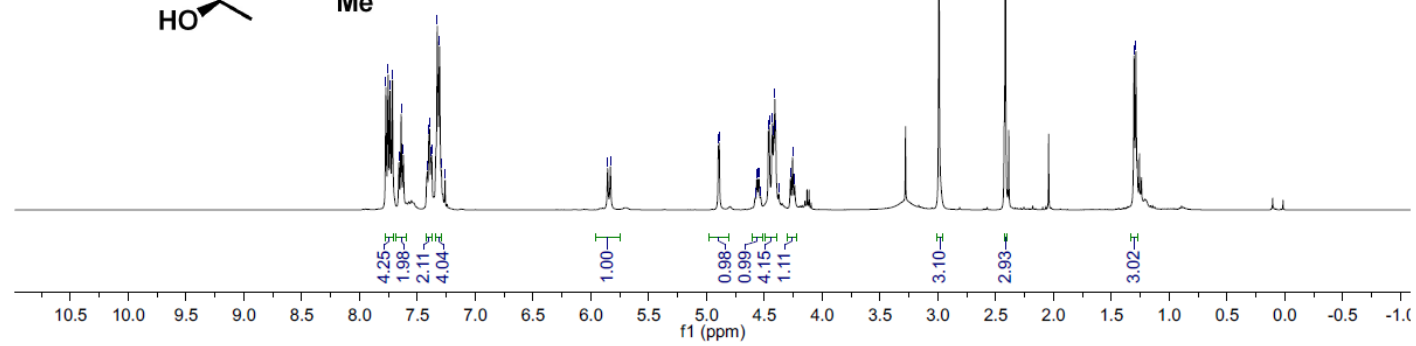

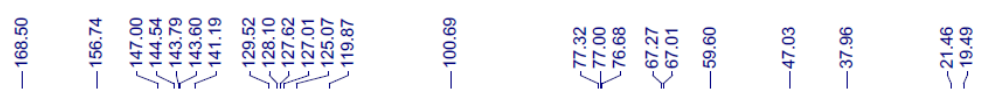

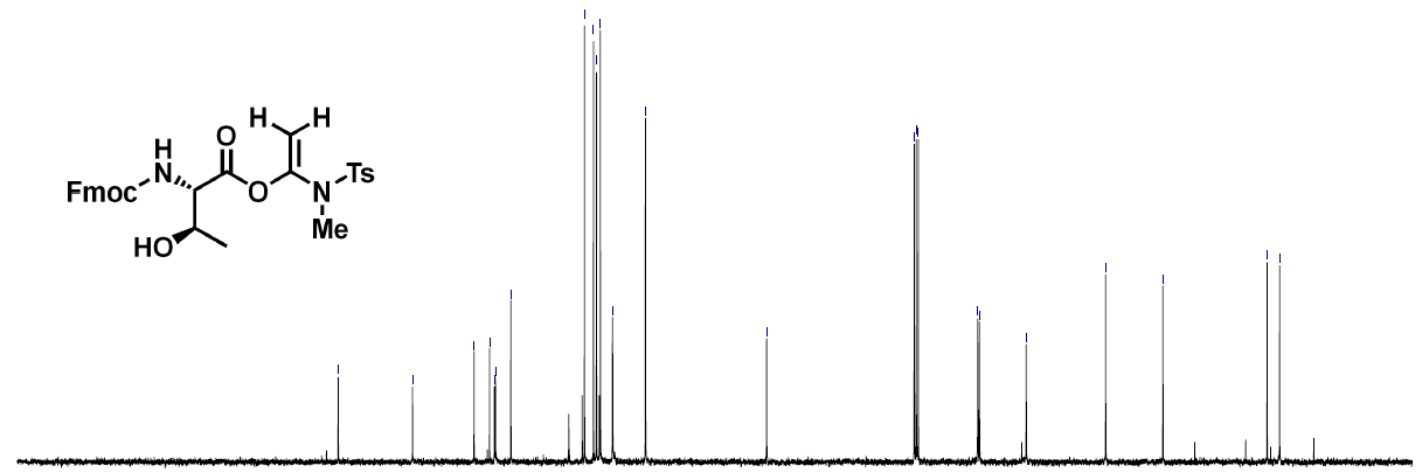

$\begin{array}{llllllllllllllllllllllll}210 & 200 & 190 & 180 & 170 & 160 & 150 & 140 & 130 & 120 & 110 & 100 & 90 & 80 & 70 & 60 & 50 & 40 & 30 & 20 & 10 & 0\end{array}$ 

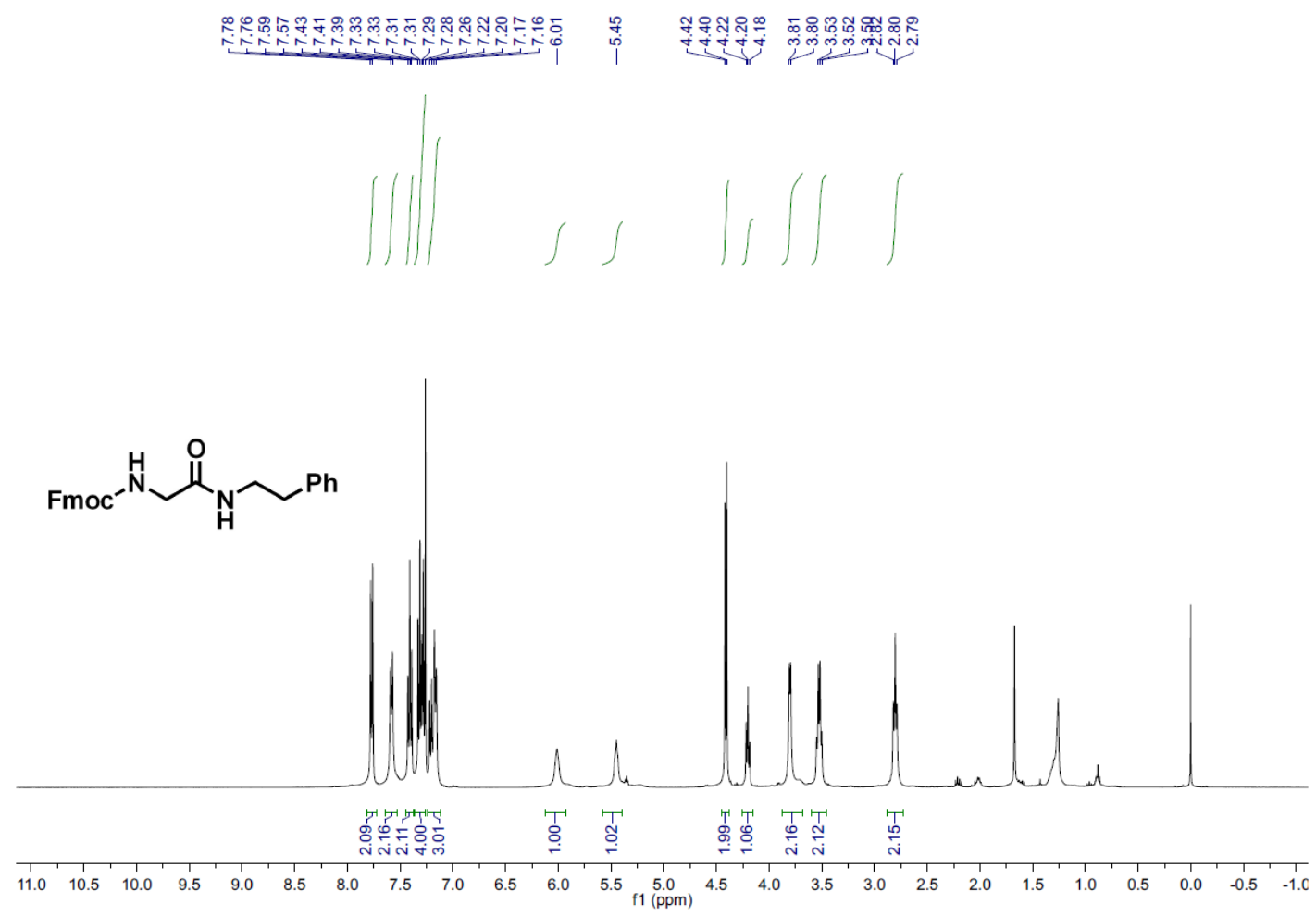

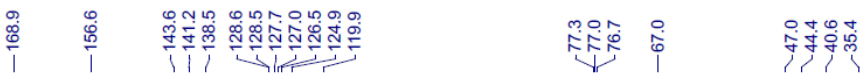

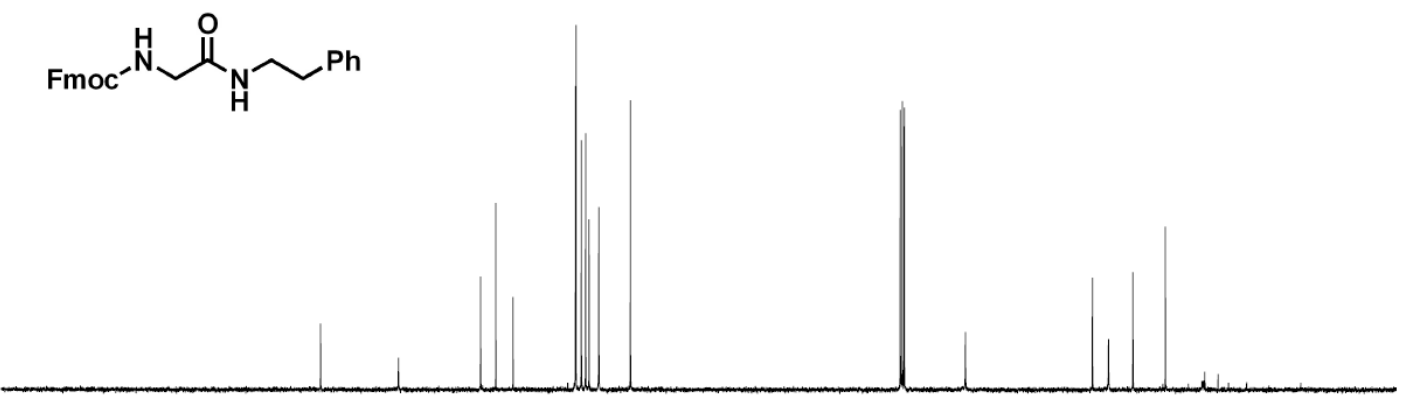

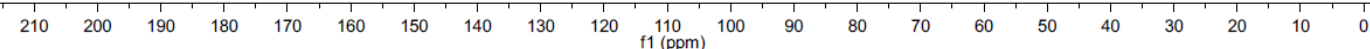



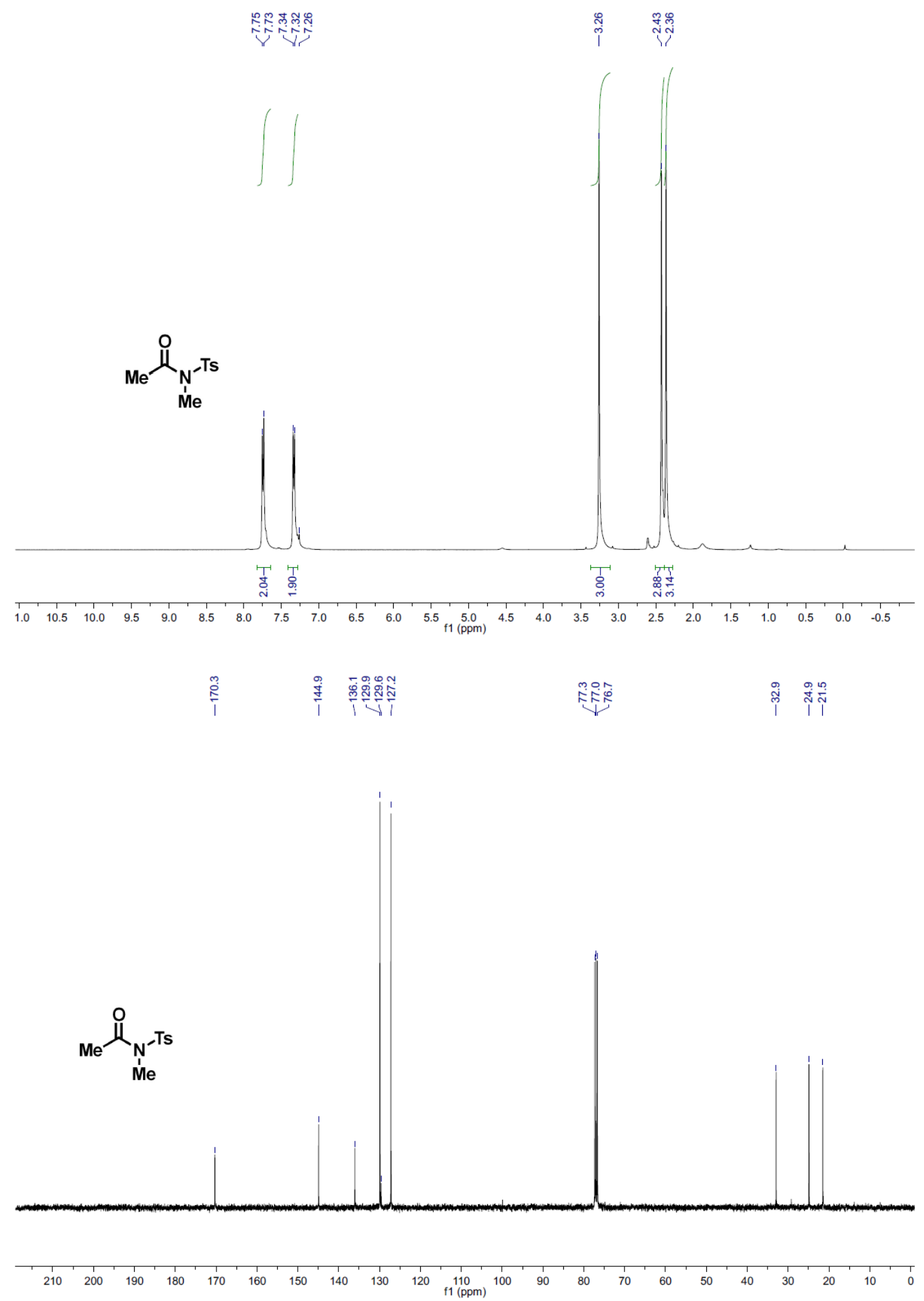


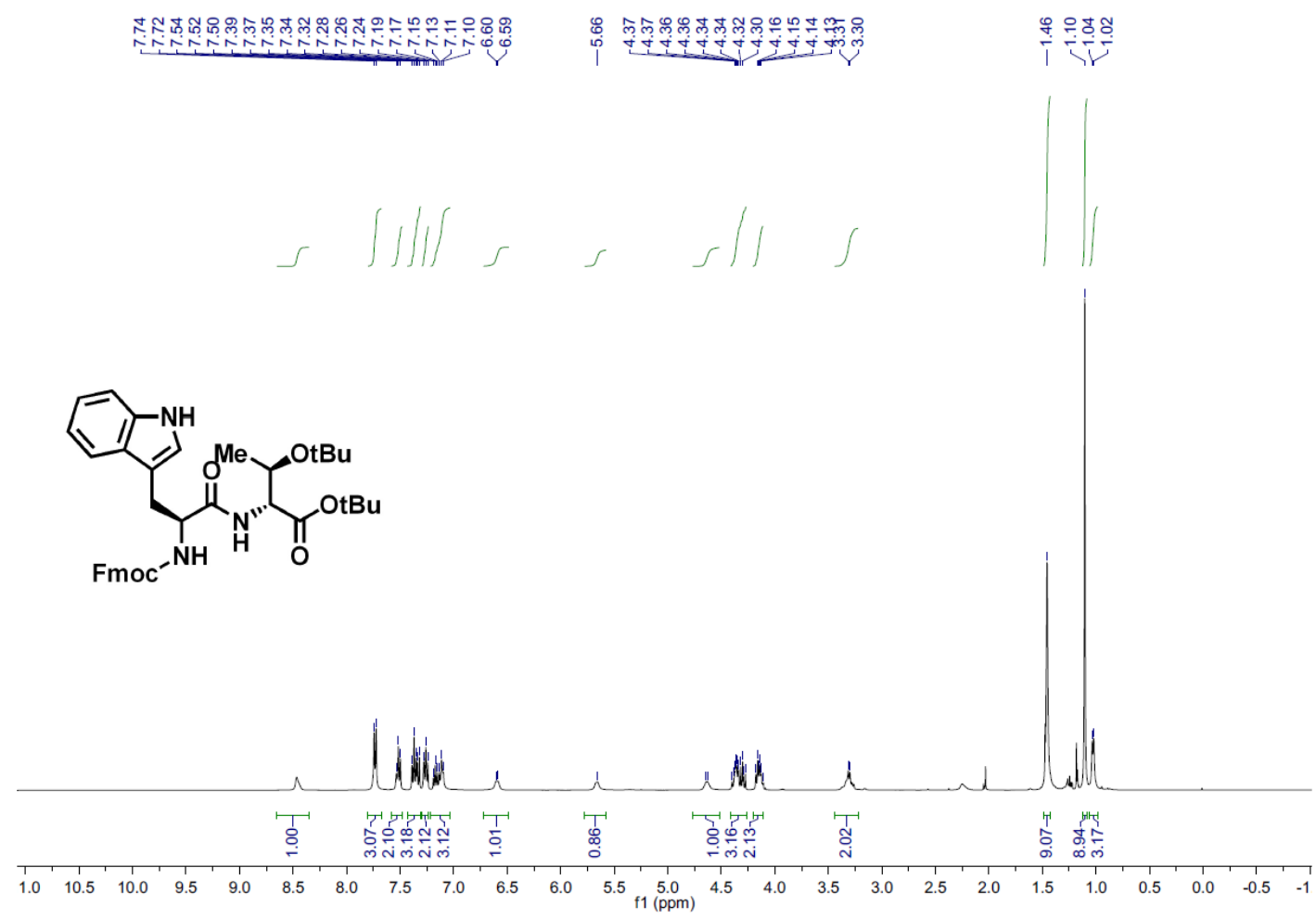

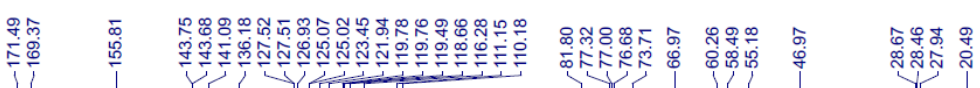
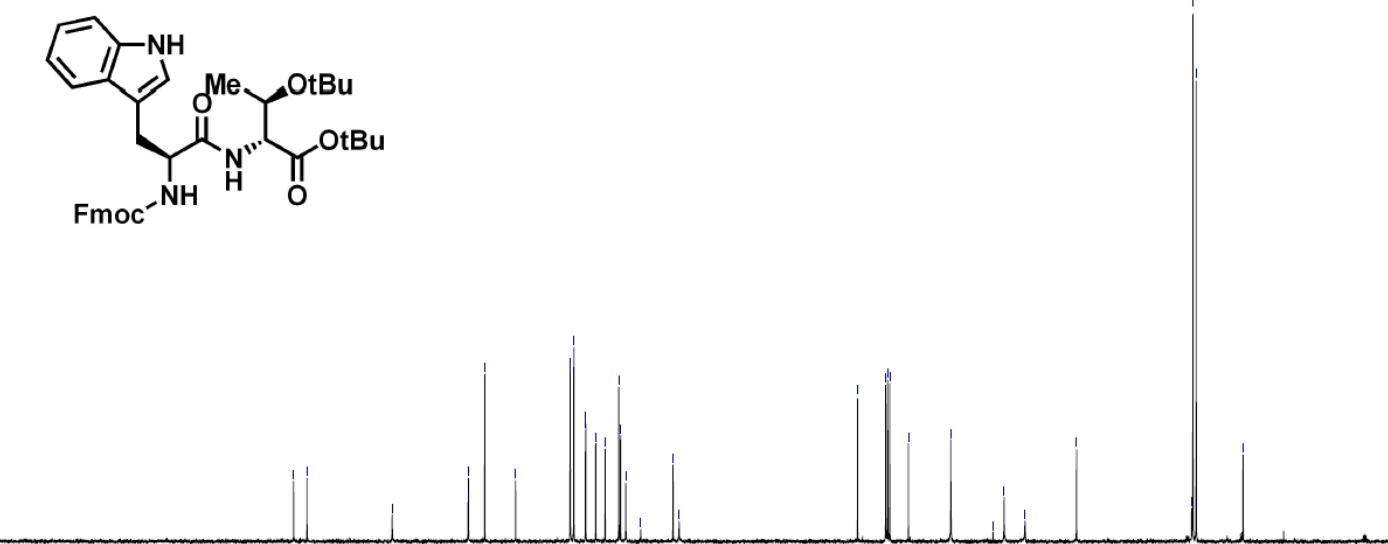

$\begin{array}{llllllllllllllllllllll}210 & 200 & 190 & 180 & 170 & 160 & 150 & 140 & 130 & 120 & 110 & 100 & 90 & 80 & 70 & 60 & 50 & 40 & 30 & 20 & 10 & 0\end{array}$ 


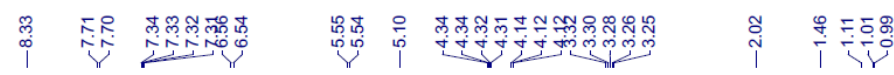

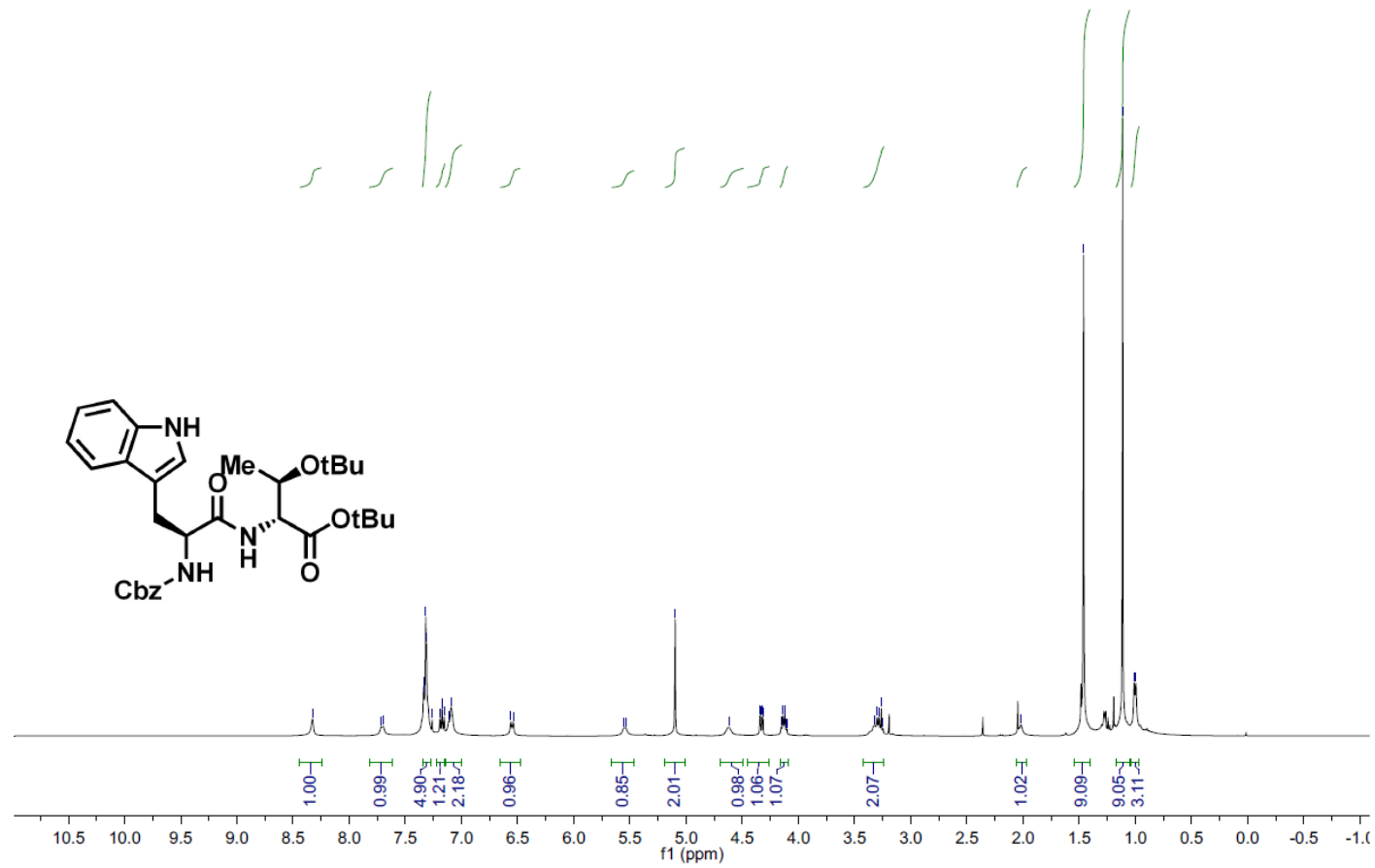

I)
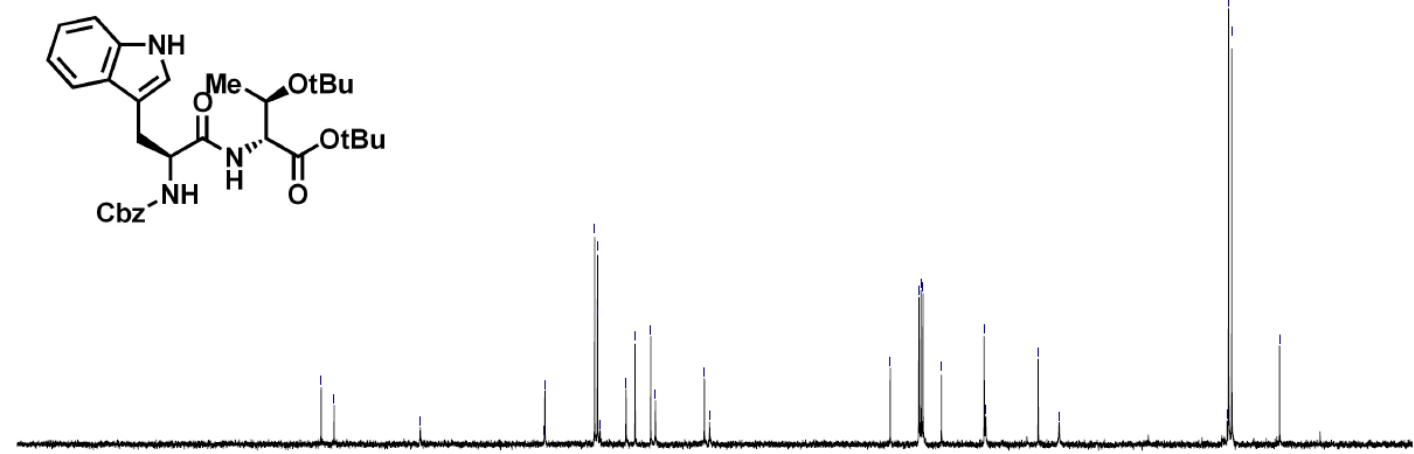

$\begin{array}{llllllllllllllllllllllllll} & 210 & 200 & 190 & 180 & 170 & 160 & 150 & 140 & 130 & 120 & 110 & 100 & 90 & 80 & 70 & 60 & 50 & 40 & 30 & 20 & 10 & 0\end{array}$ 


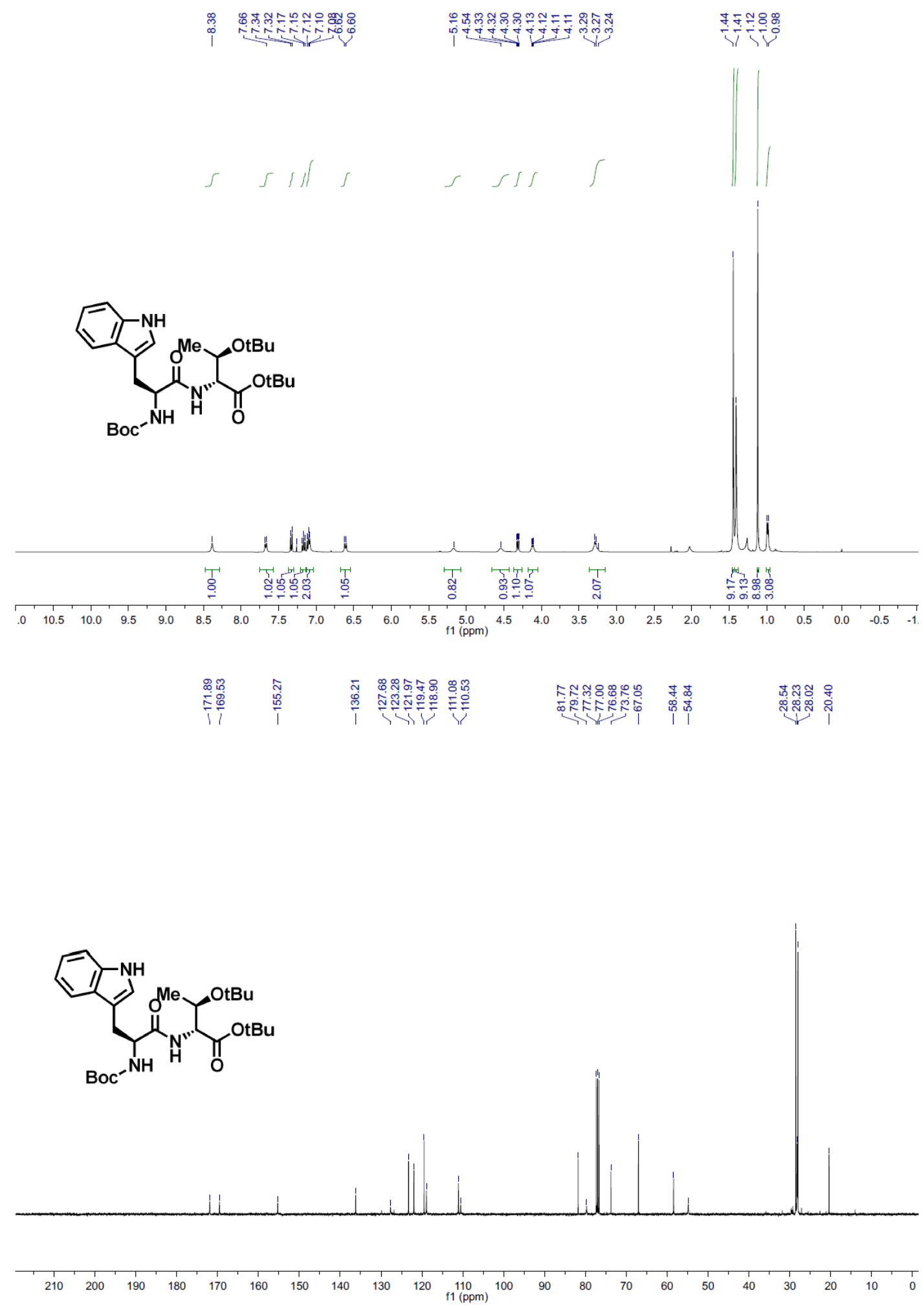




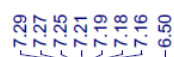

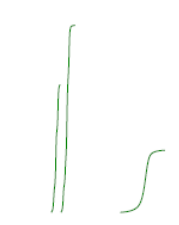

策

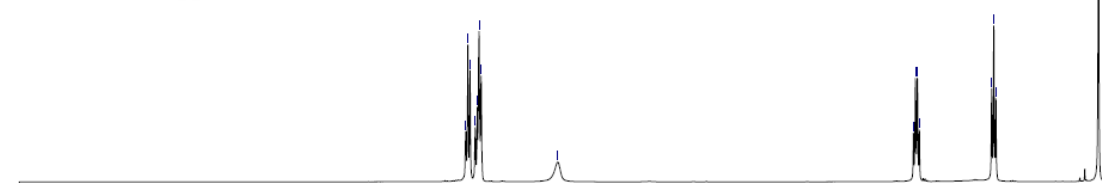

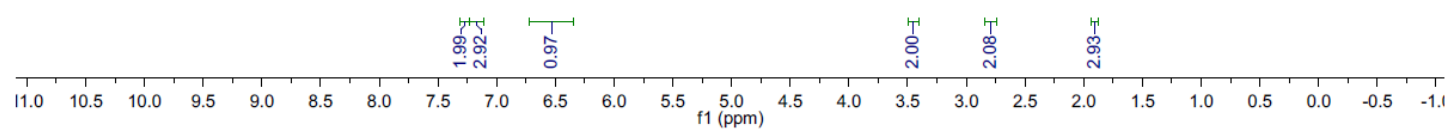

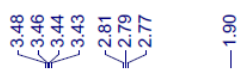
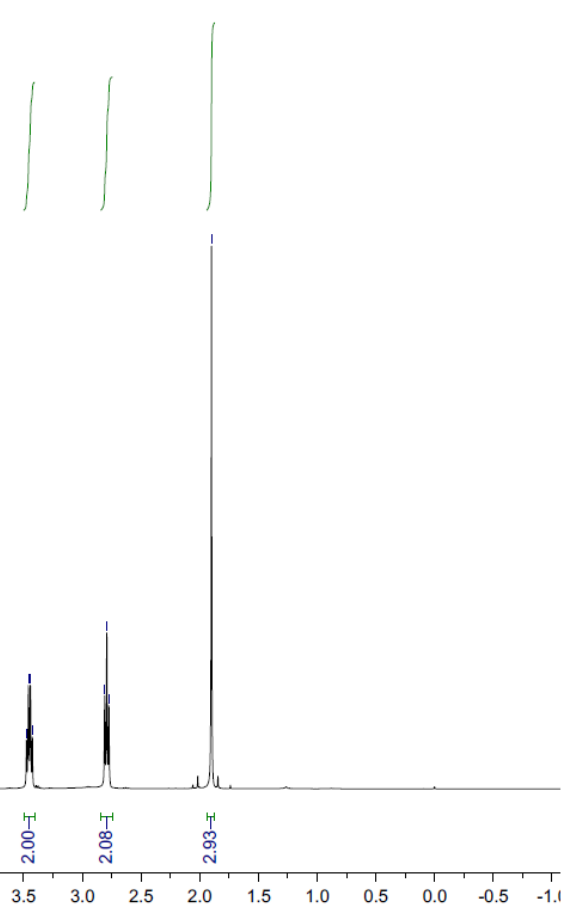

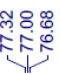

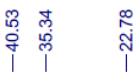

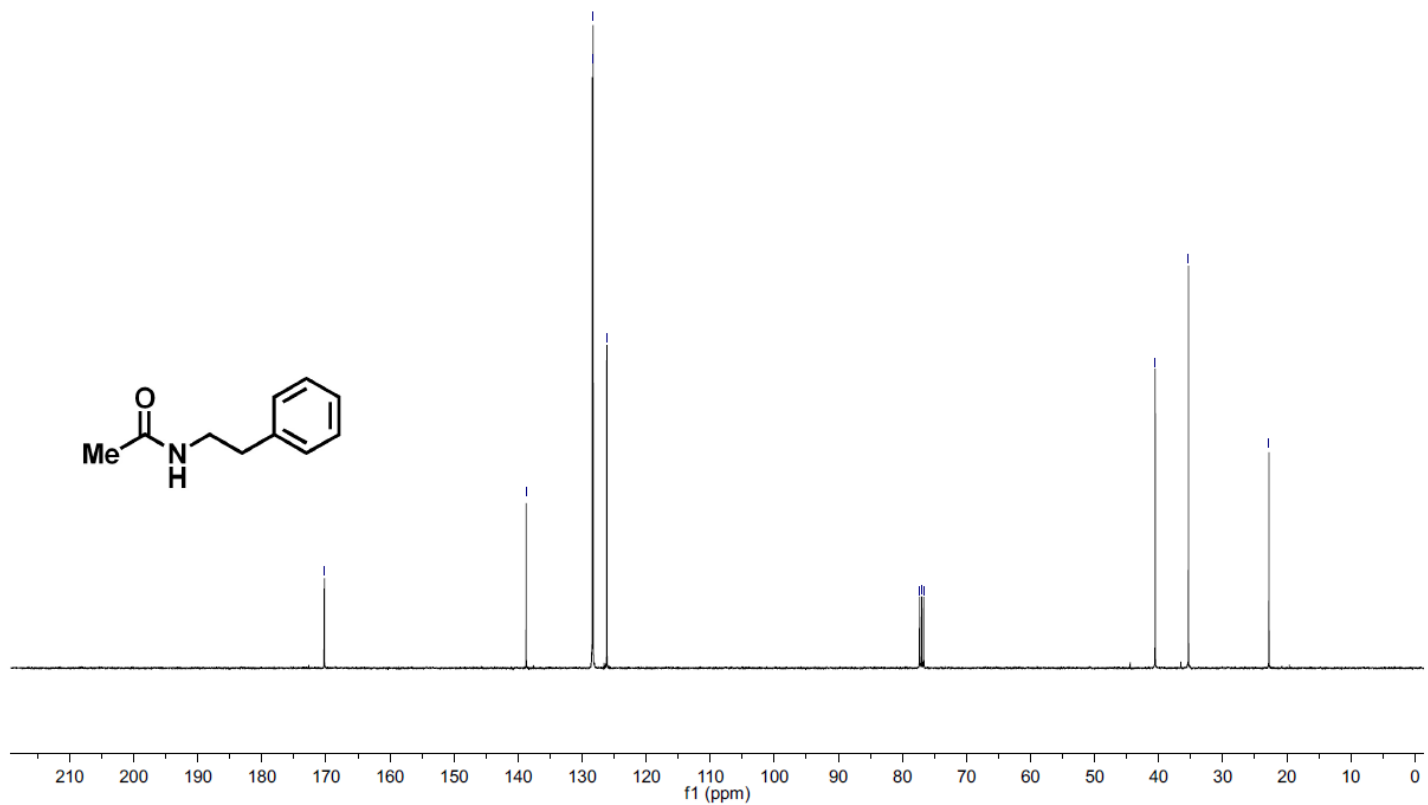



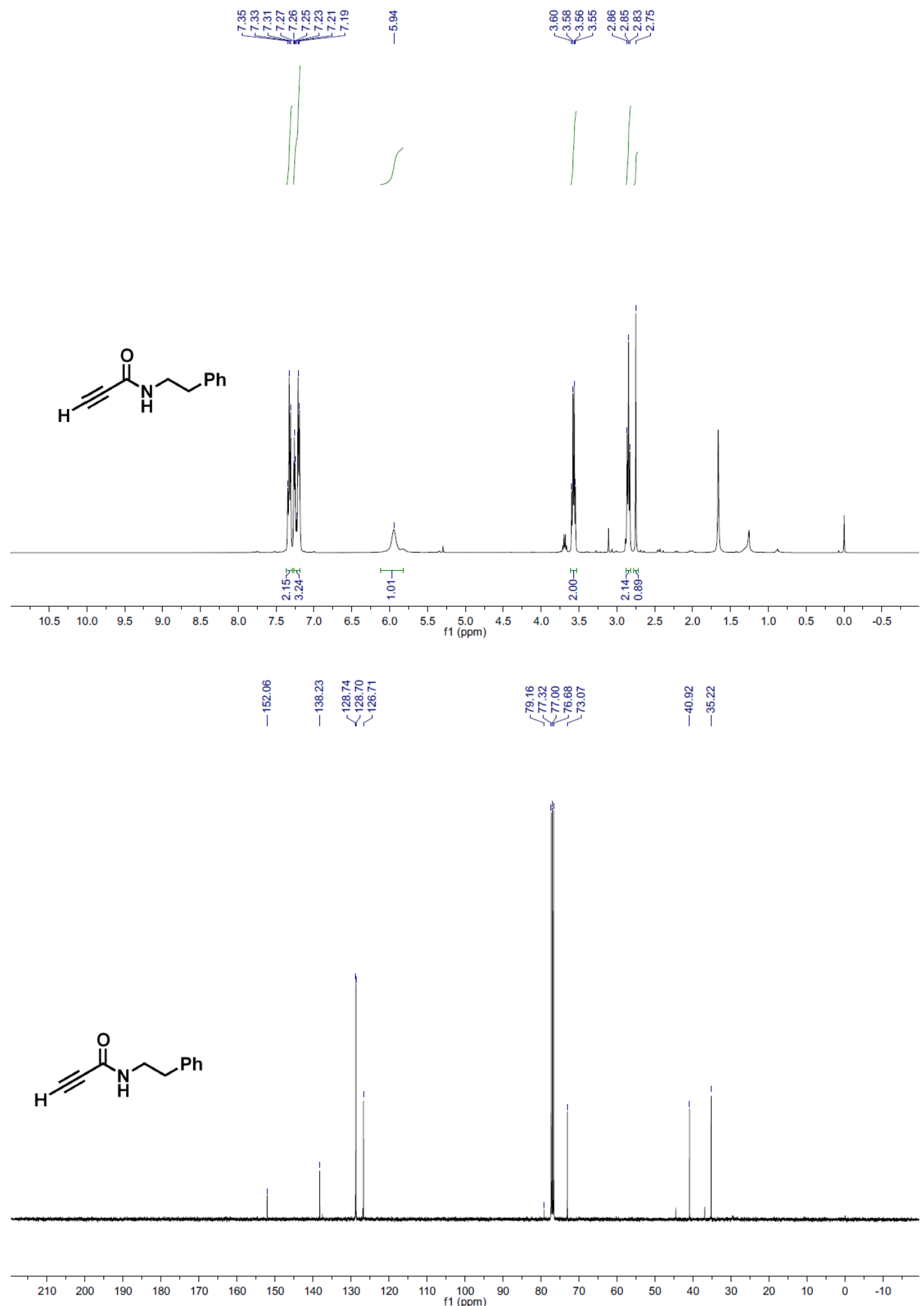

NरN尔

| 

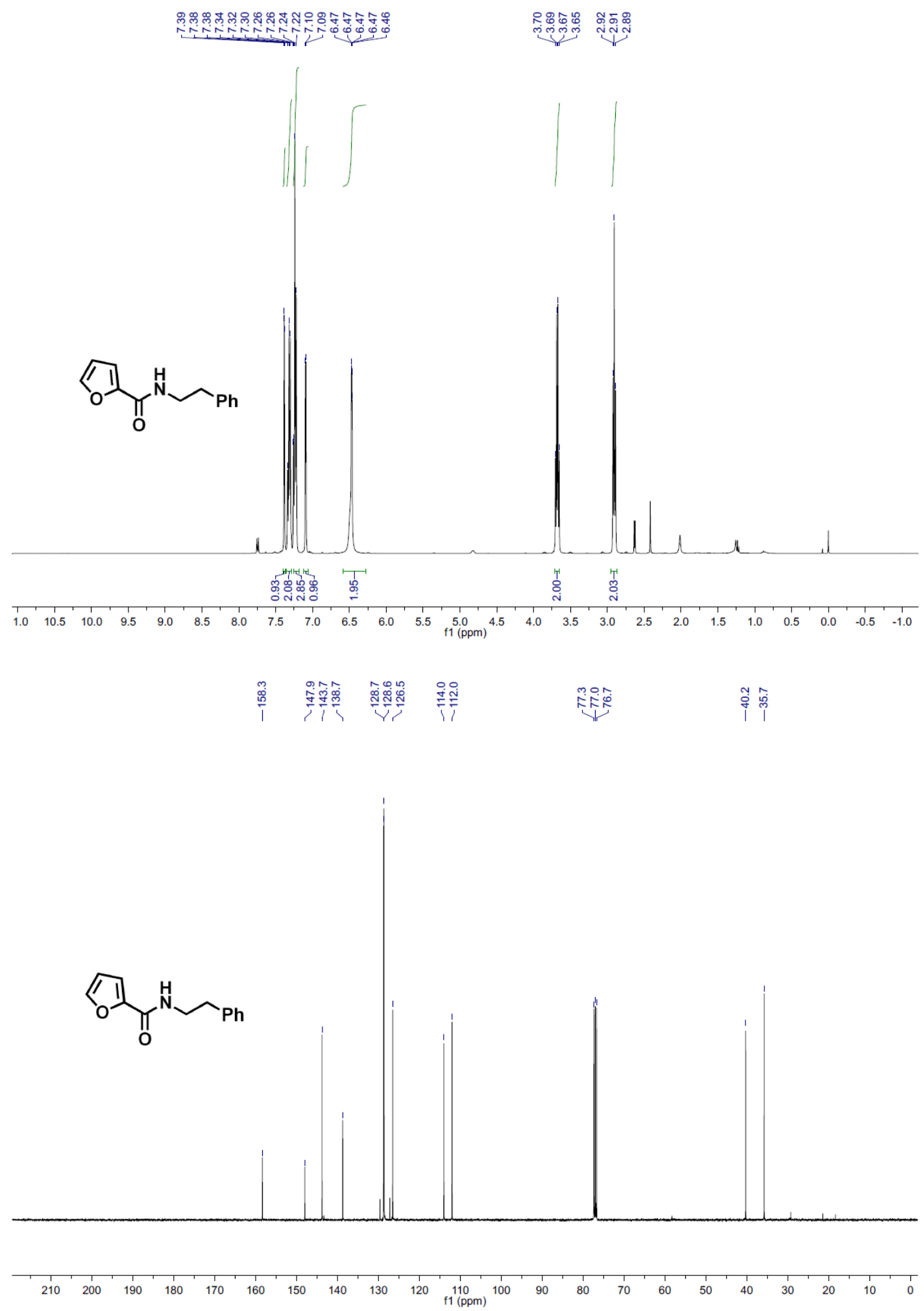

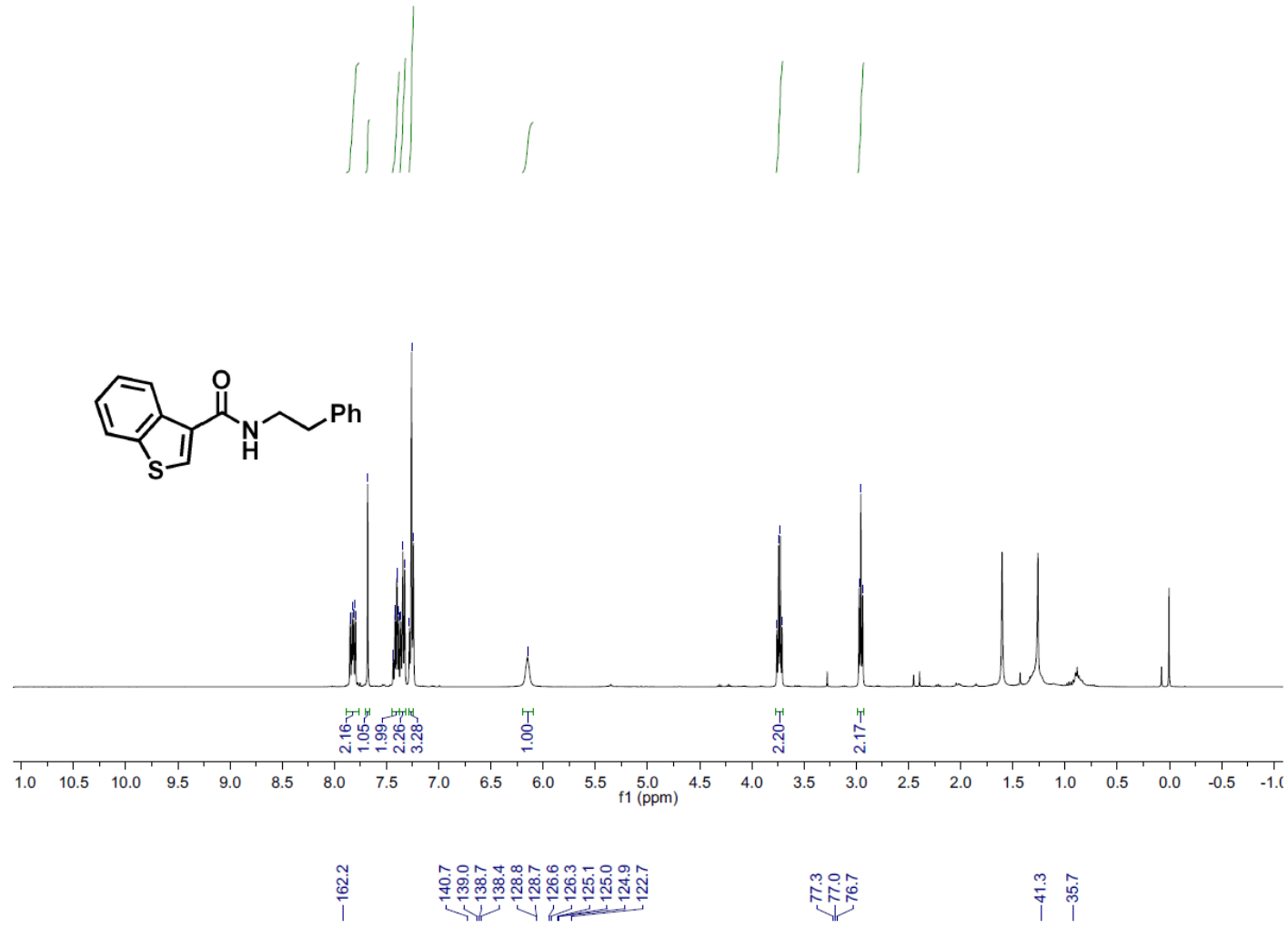

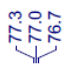

$\stackrel{m}{\dot{j}} \hat{i}$
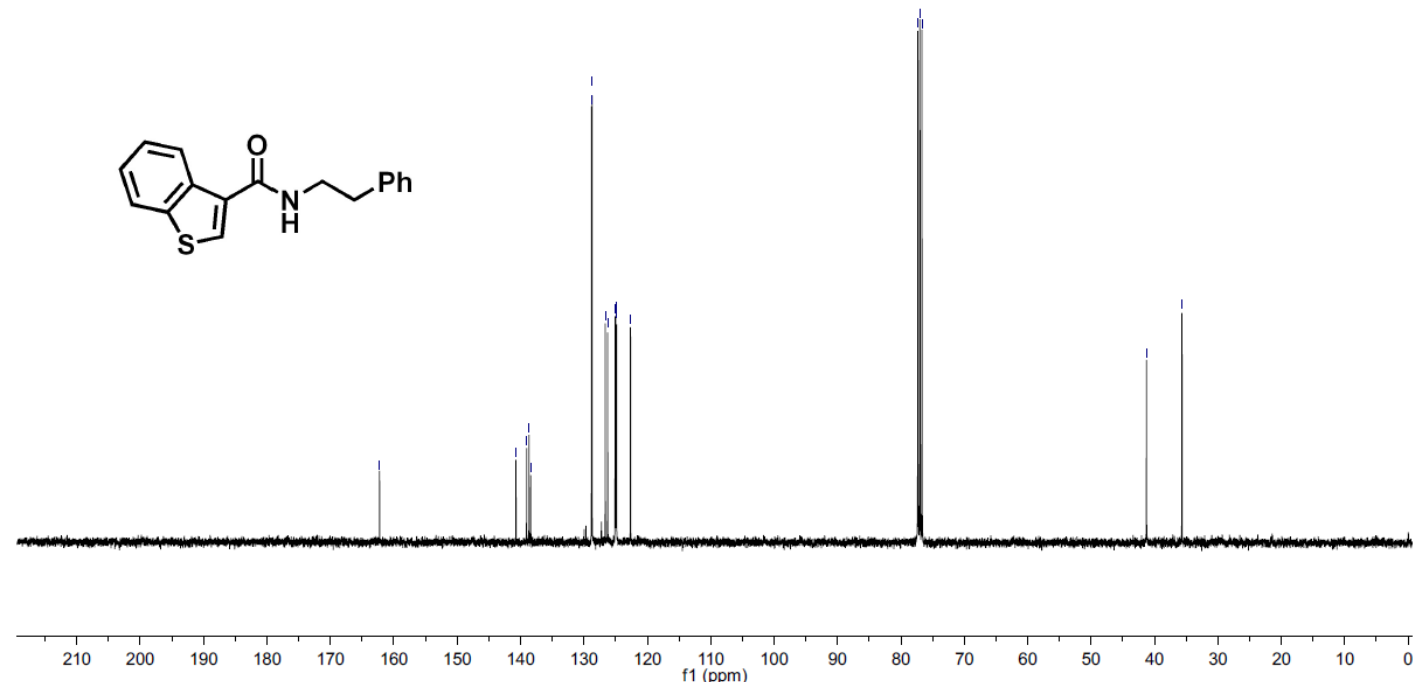


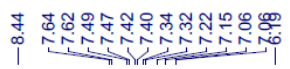

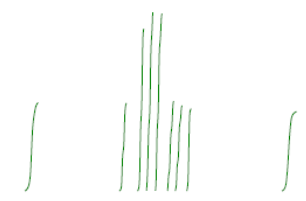

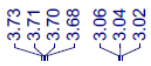

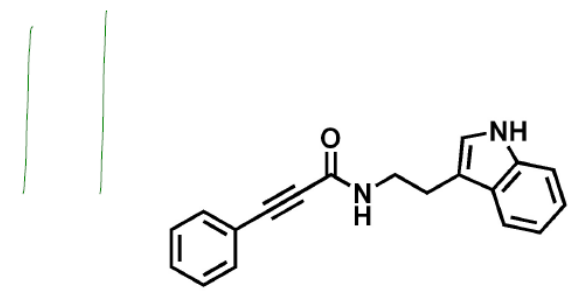

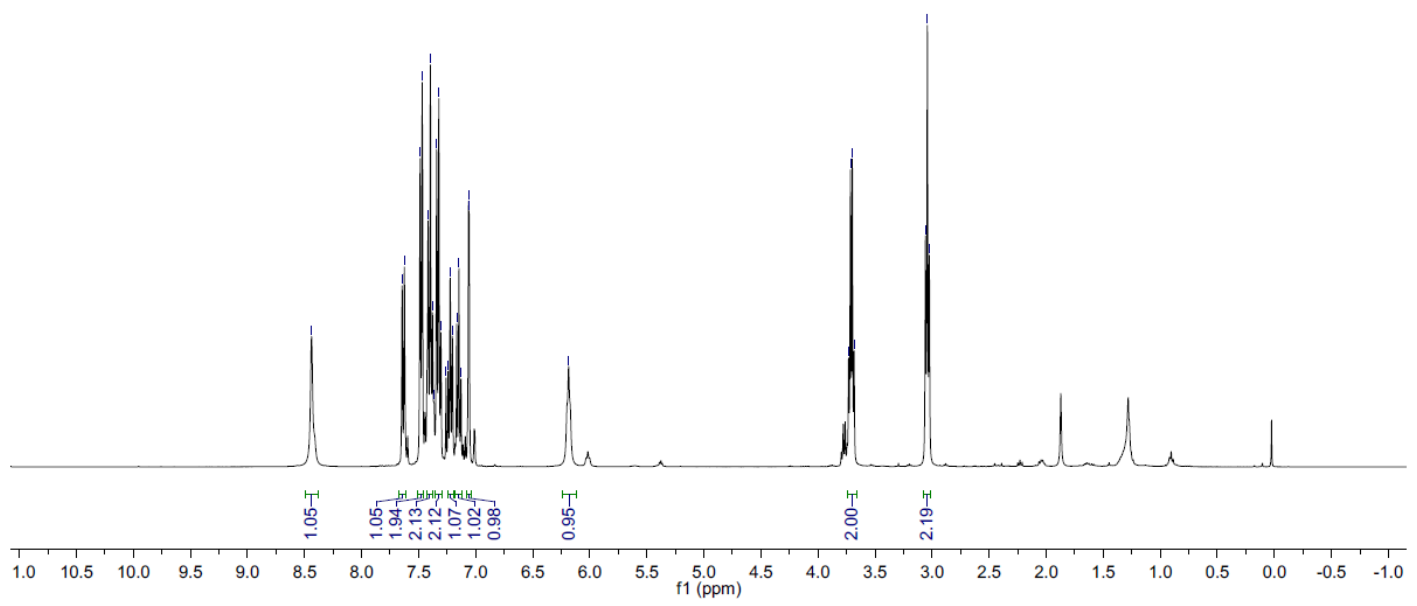

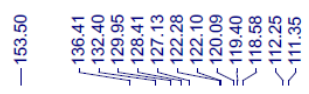

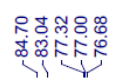

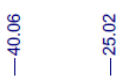
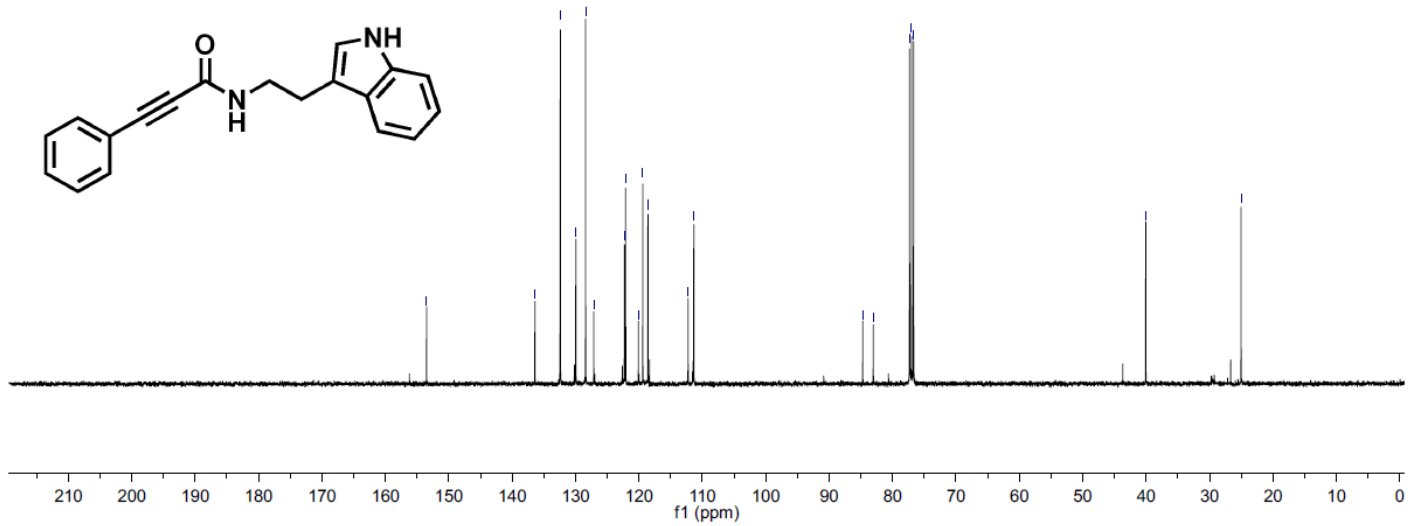

S85 

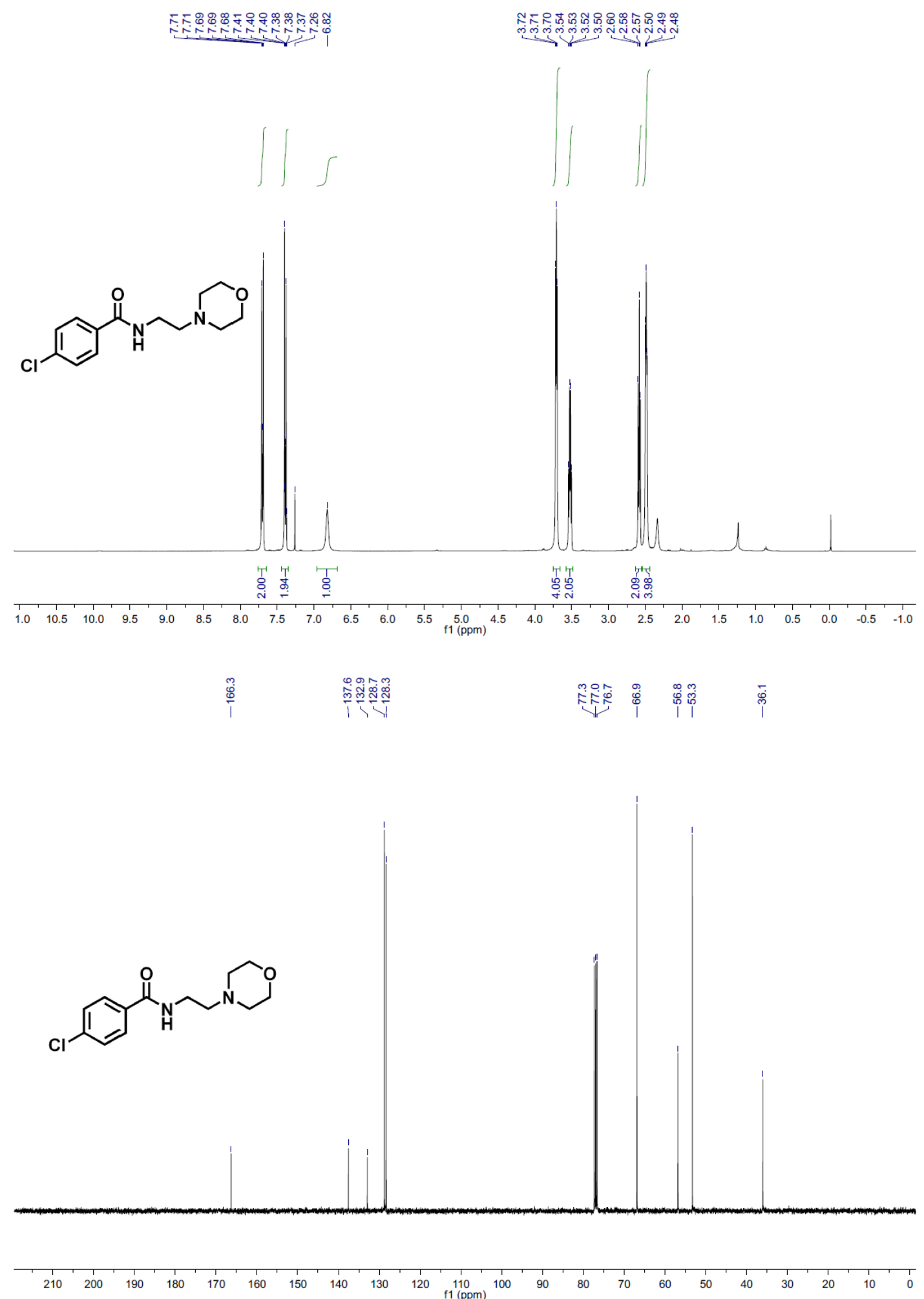

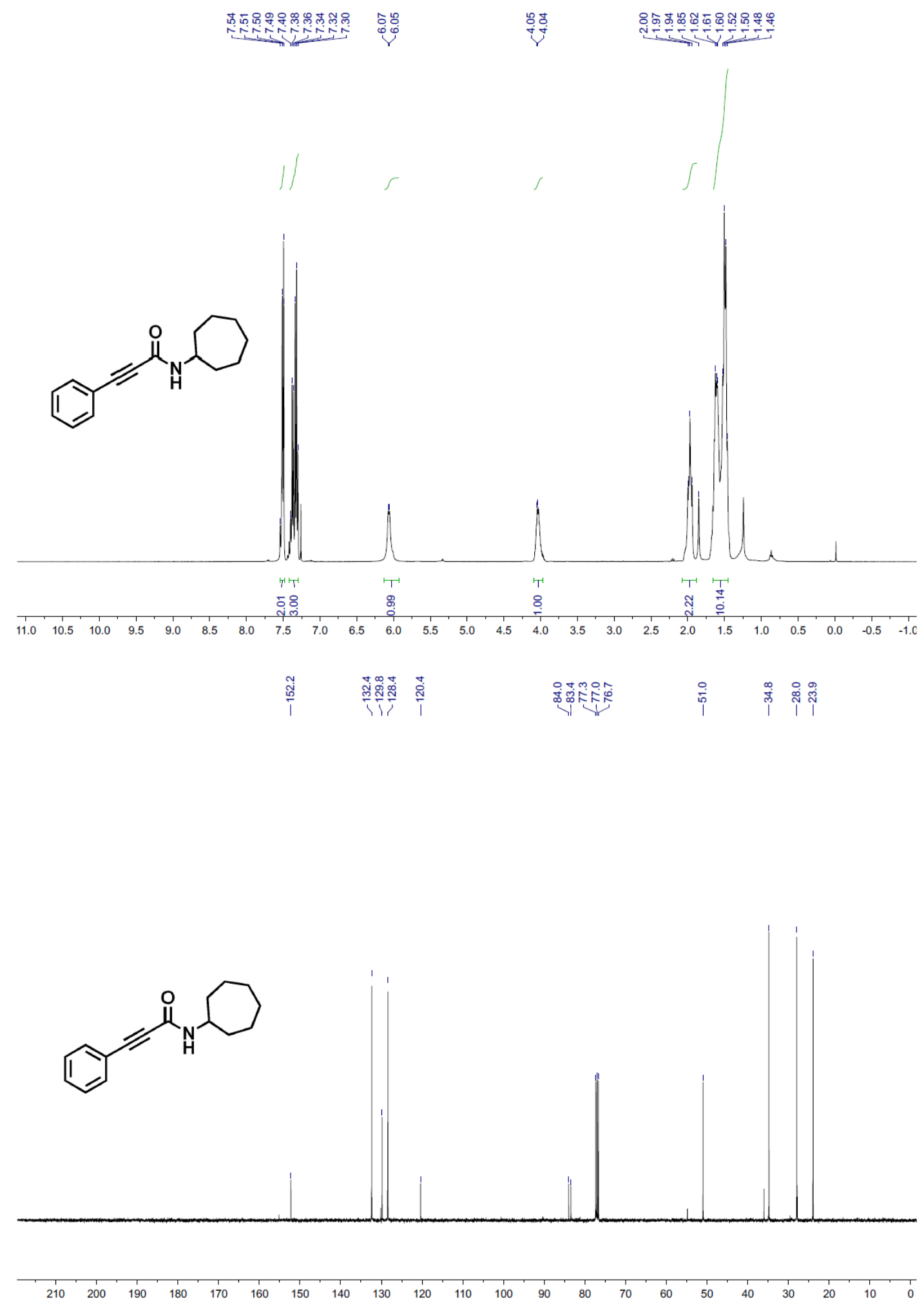

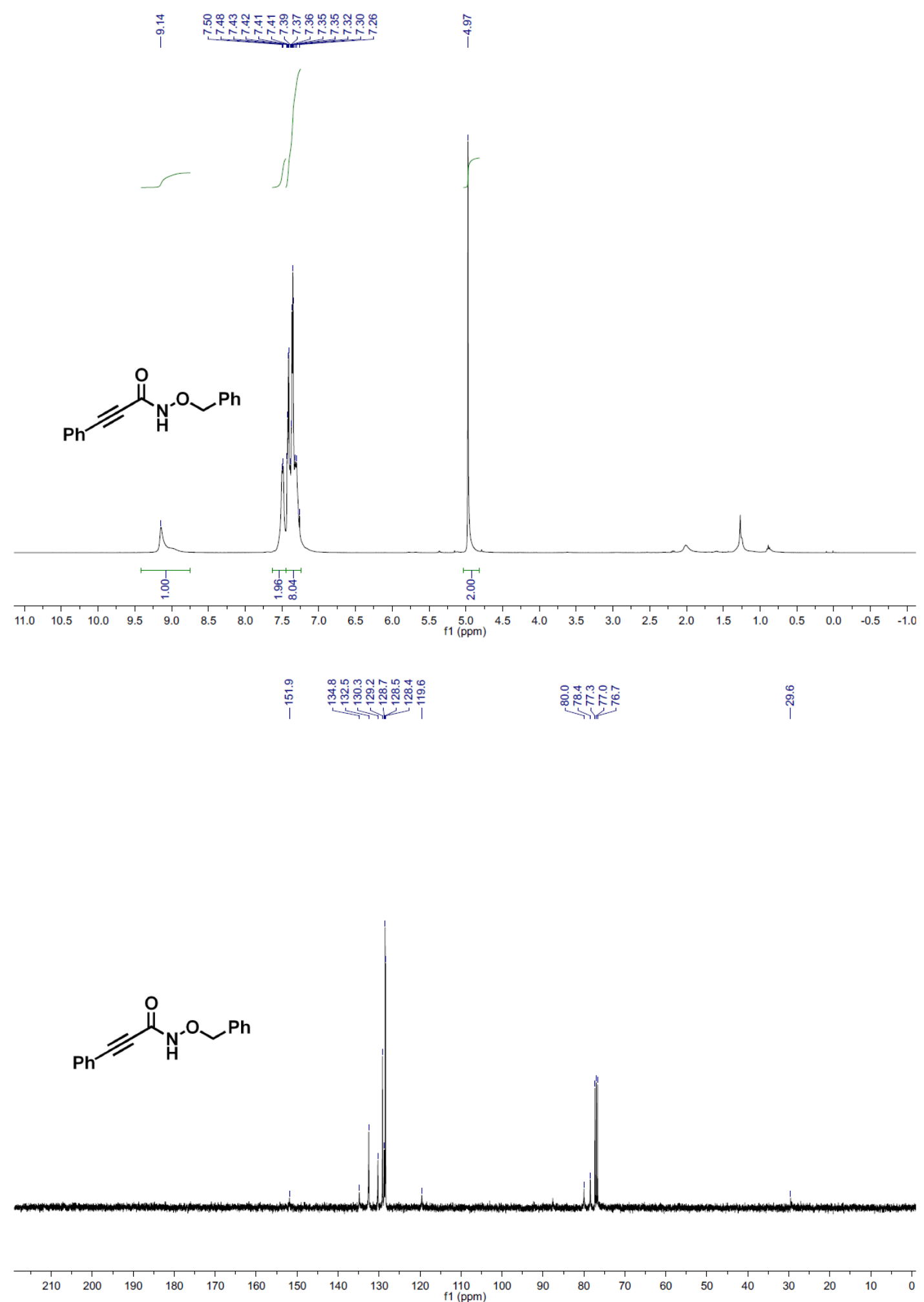
గִ

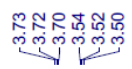

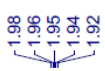
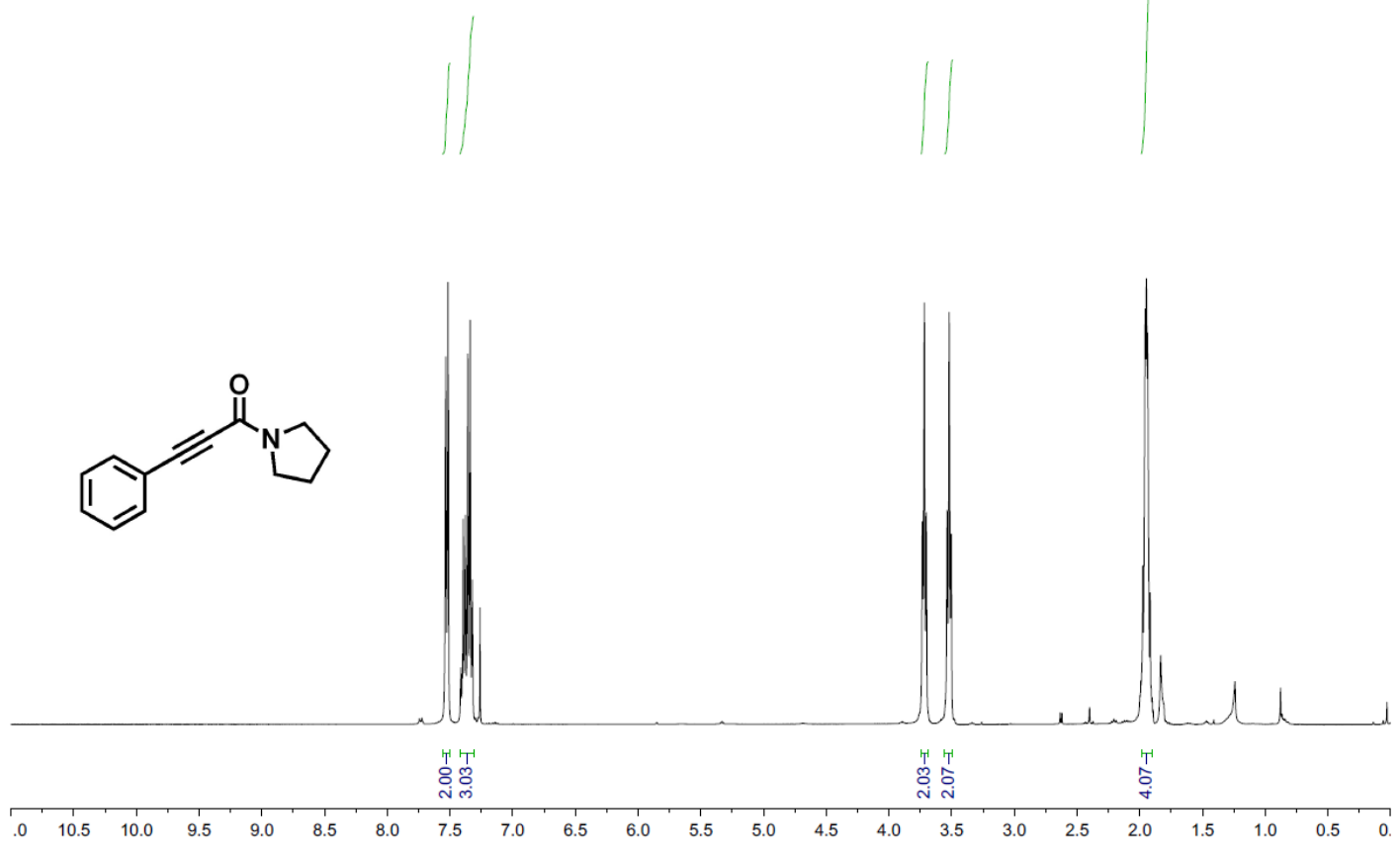

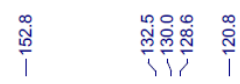

$\begin{array}{cc}\infty & \infty \\ \infty & \infty \\ \infty & \infty \\ 1 & 1\end{array}$

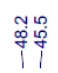

ฟึำ

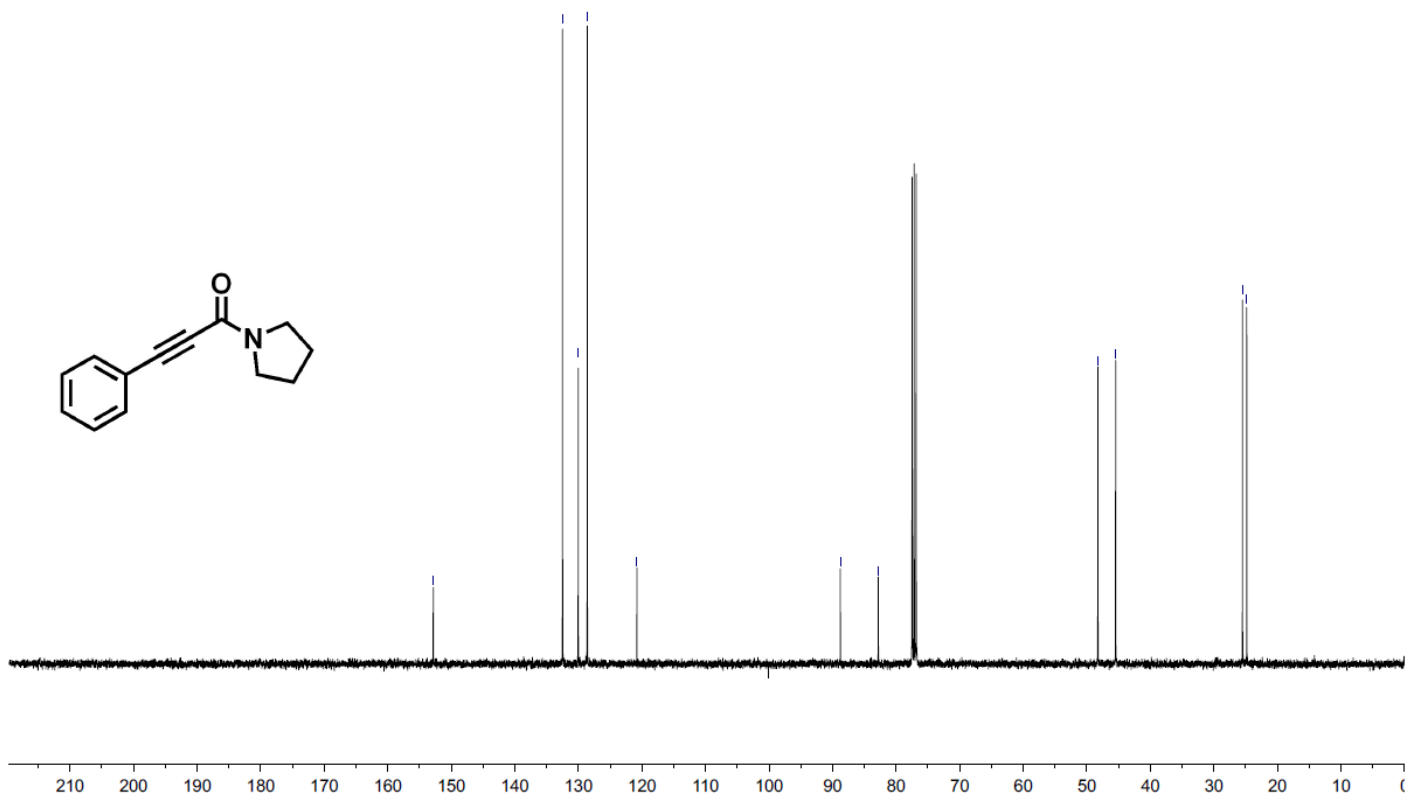

S89 


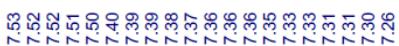
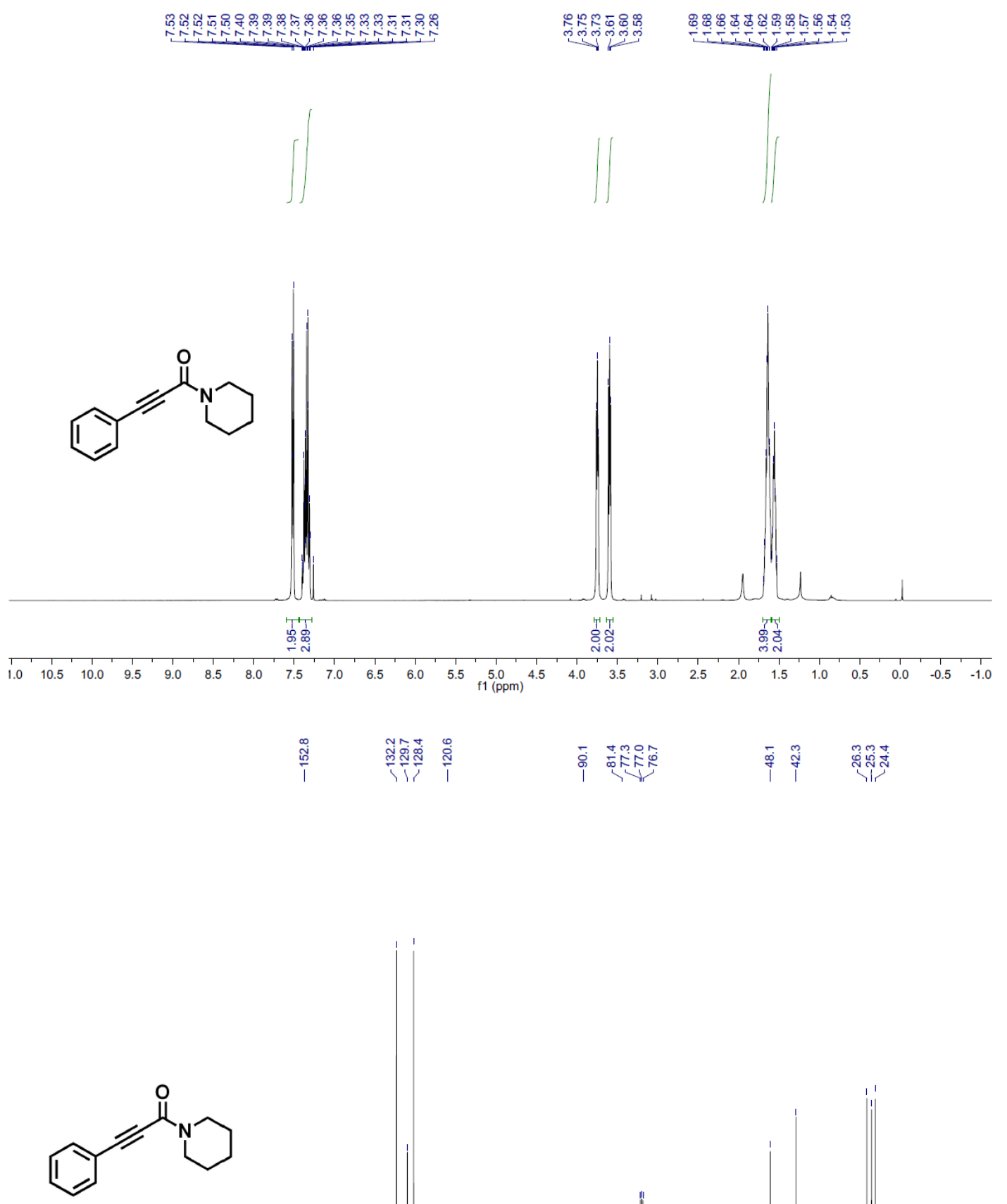

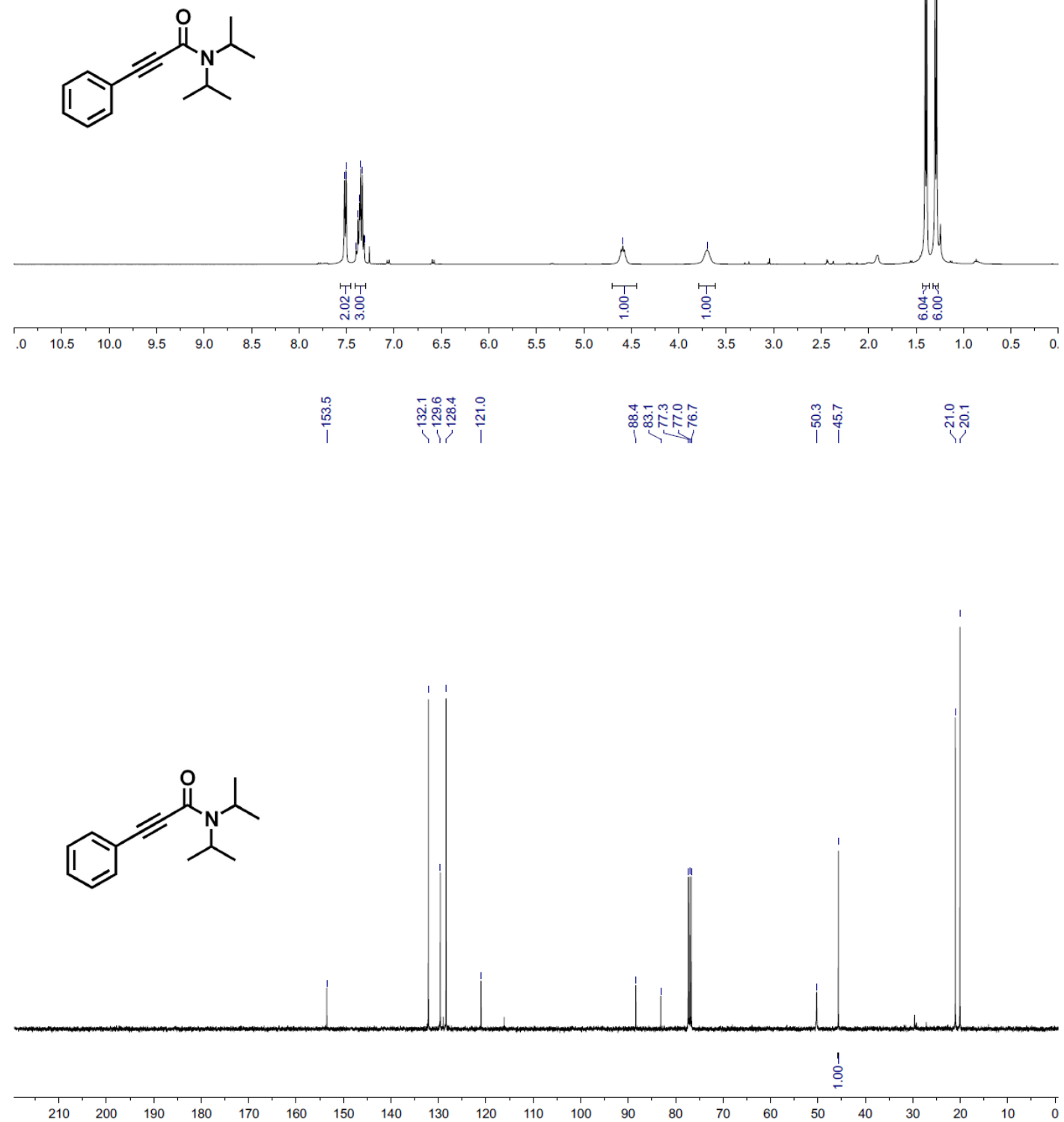


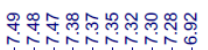

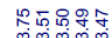

||
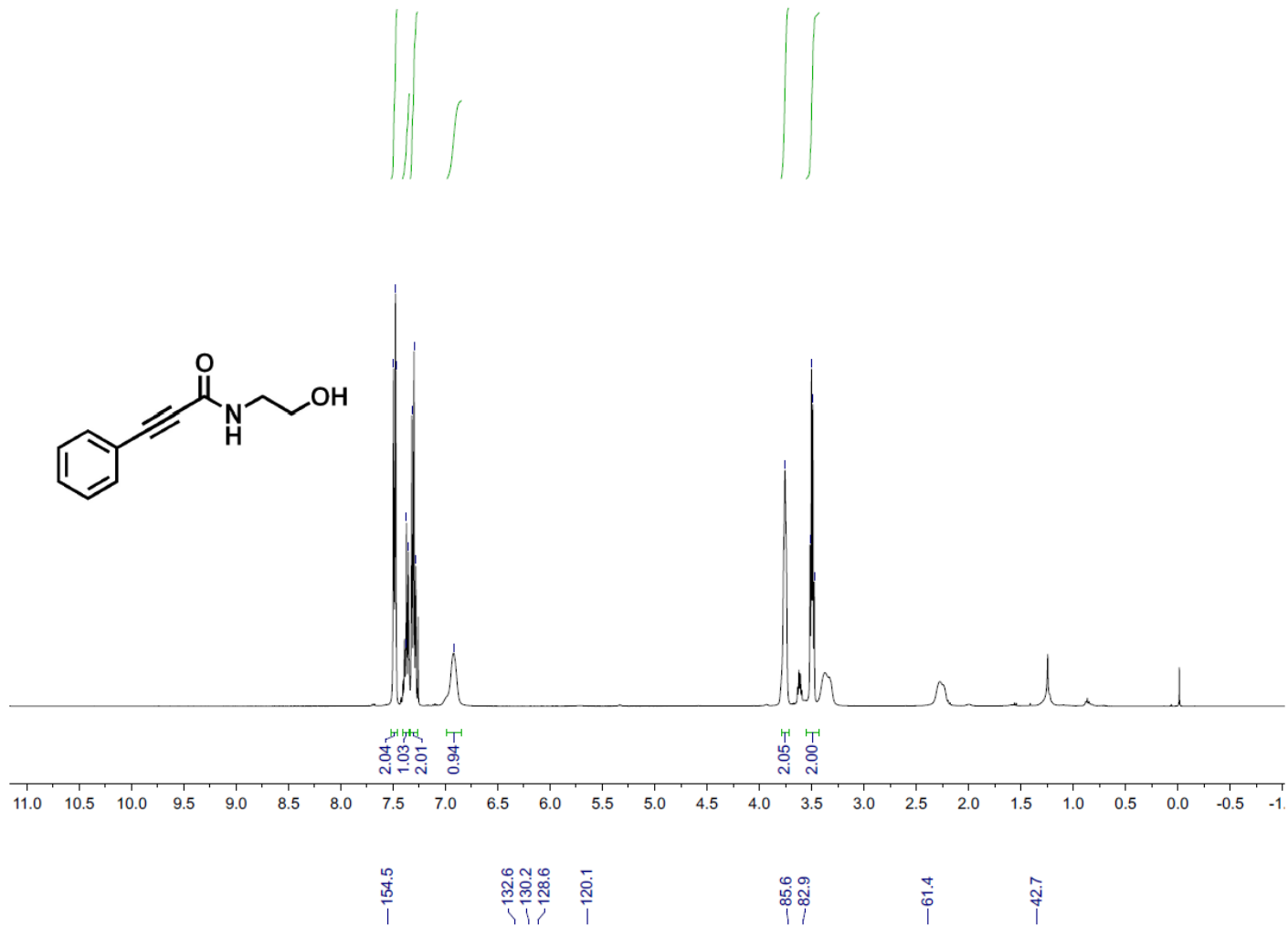

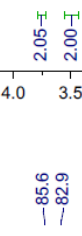
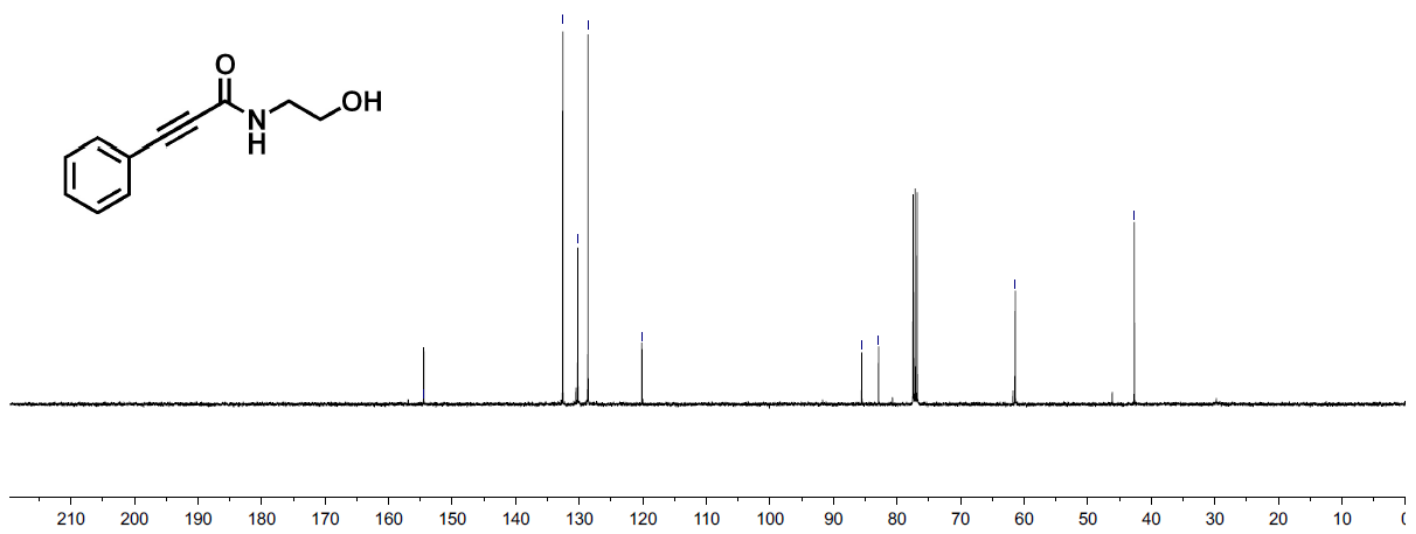

S92 

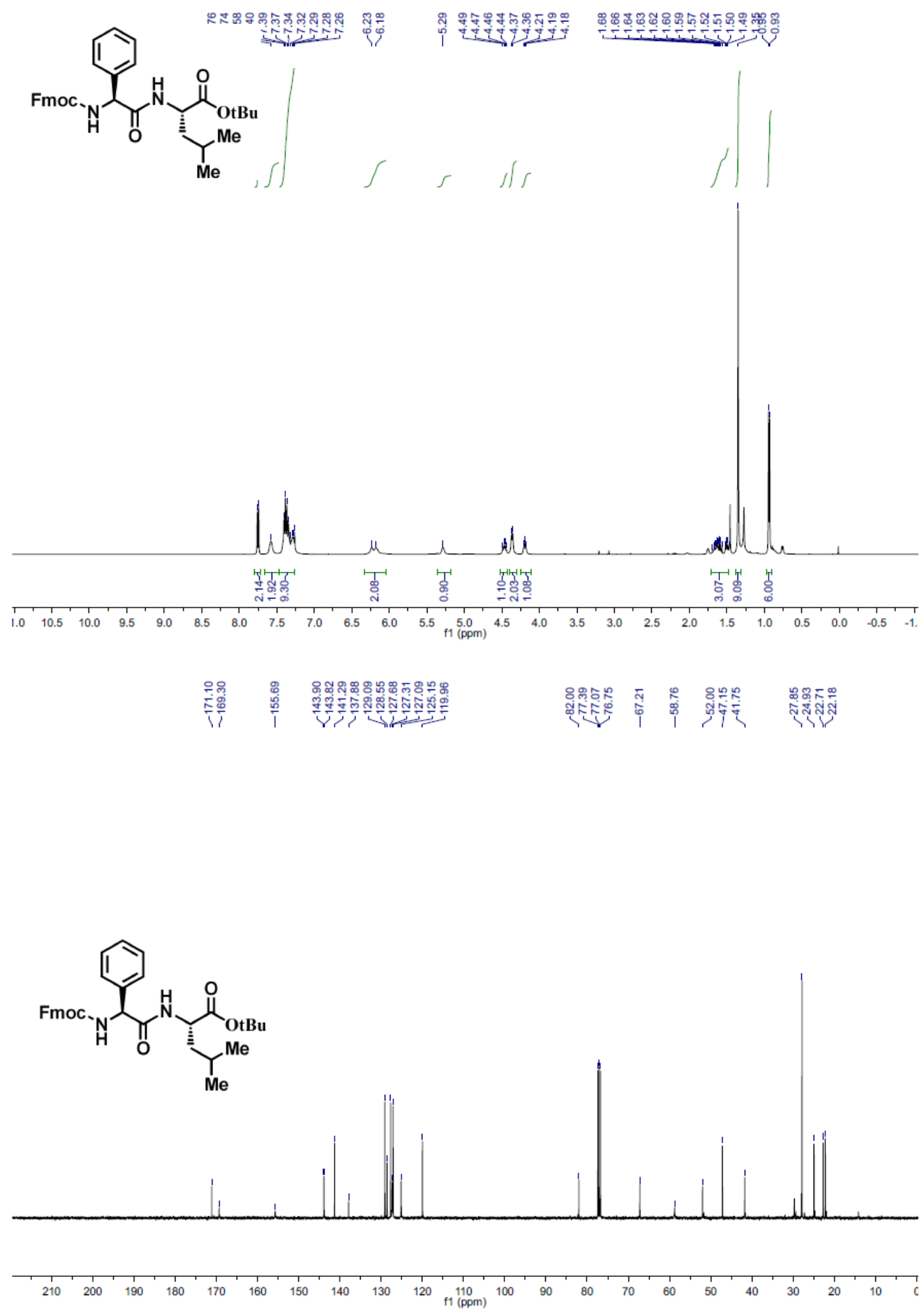

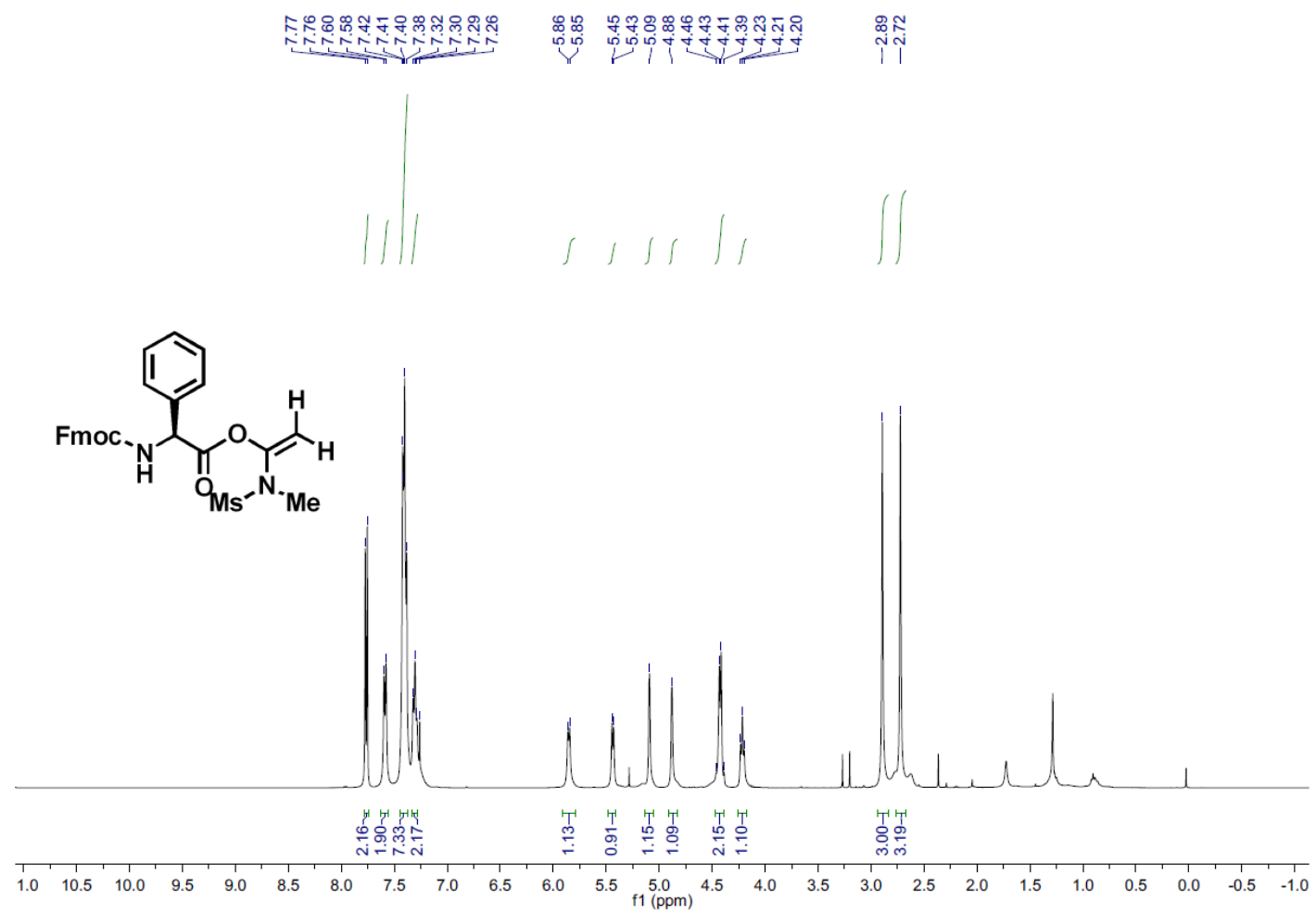

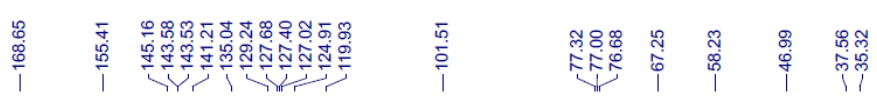

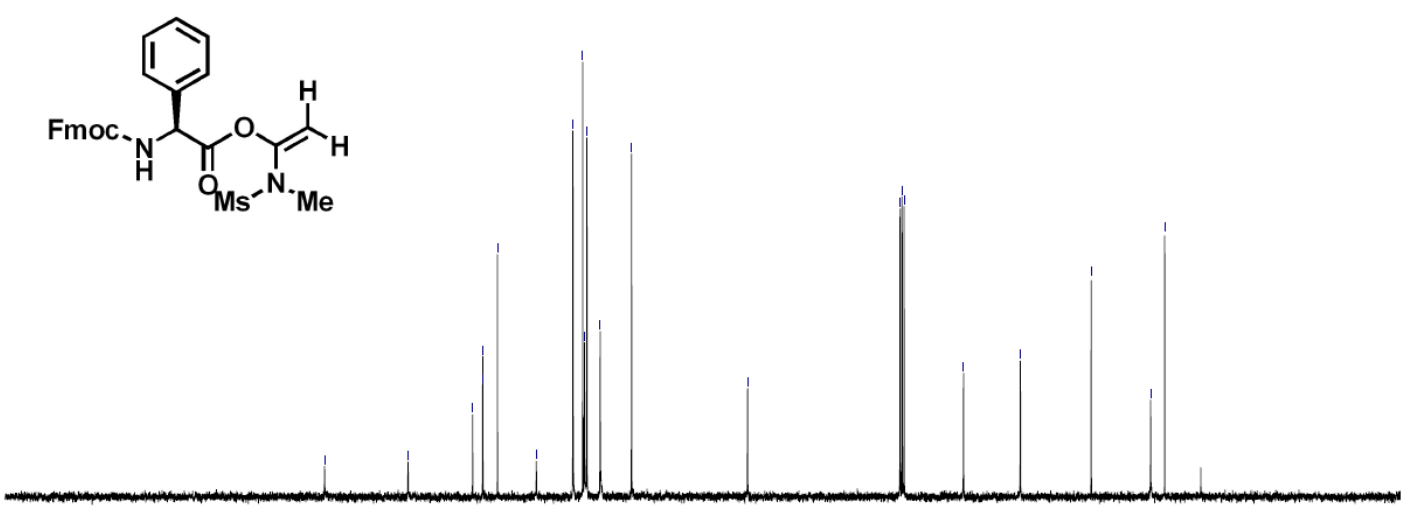

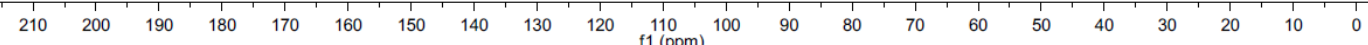




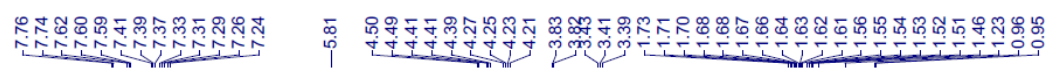
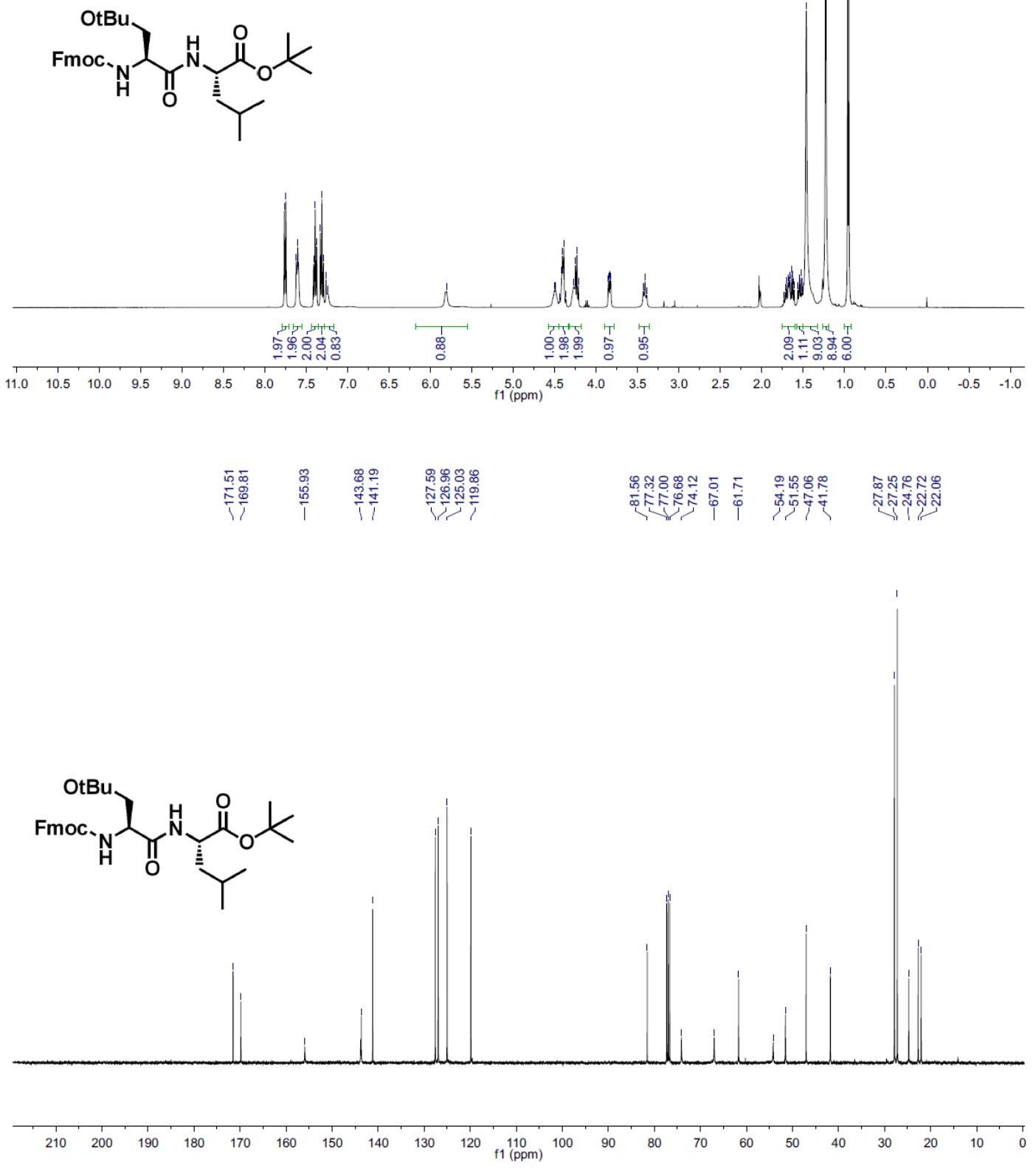

S95 


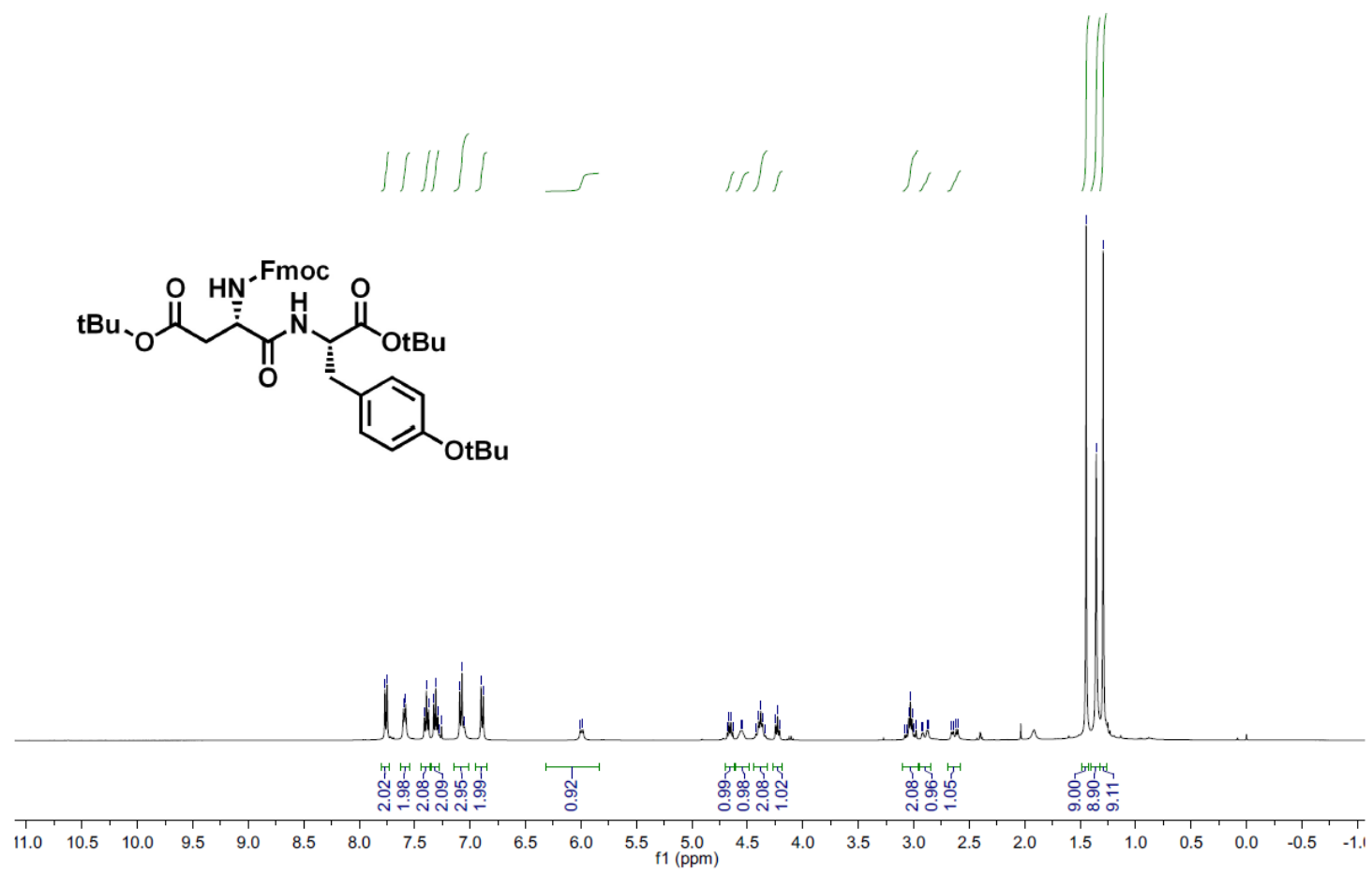

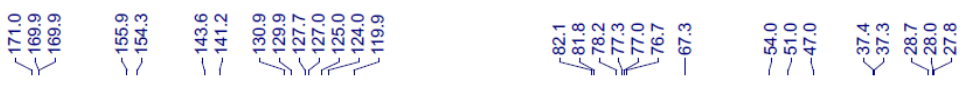

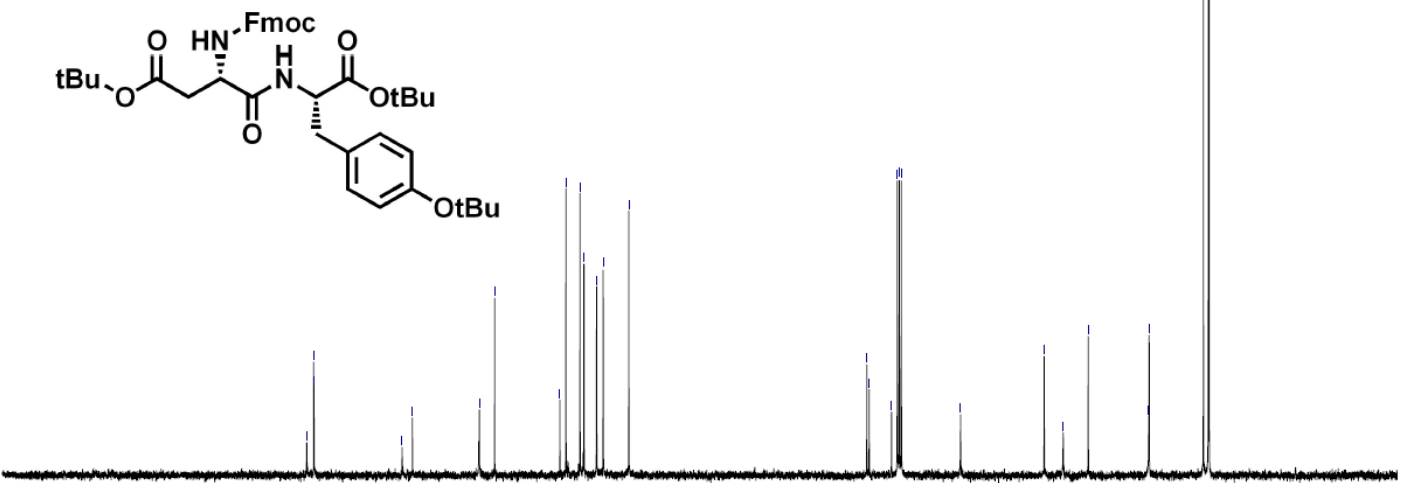

$\begin{array}{llllllllllllllllllllllllll}210 & 200 & 190 & 180 & 170 & 160 & 150 & 140 & 130 & 120 & 110 & 100 & 90 & 80 & 70 & 60 & 50 & 40 & 30 & 20 & 10 & 0\end{array}$ 


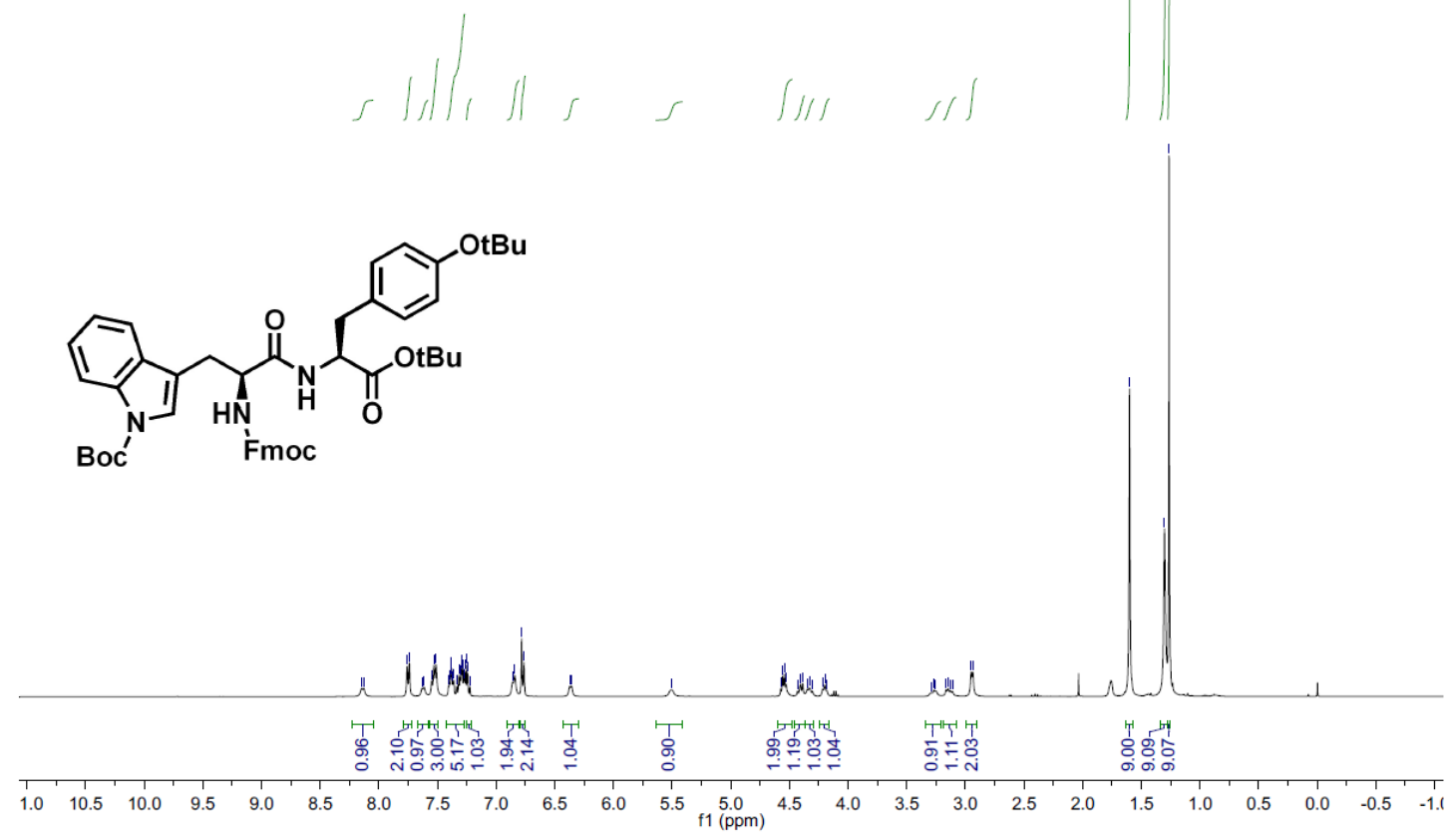

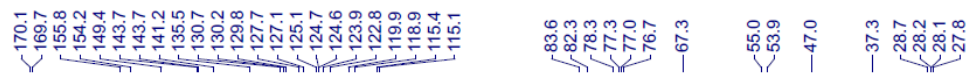

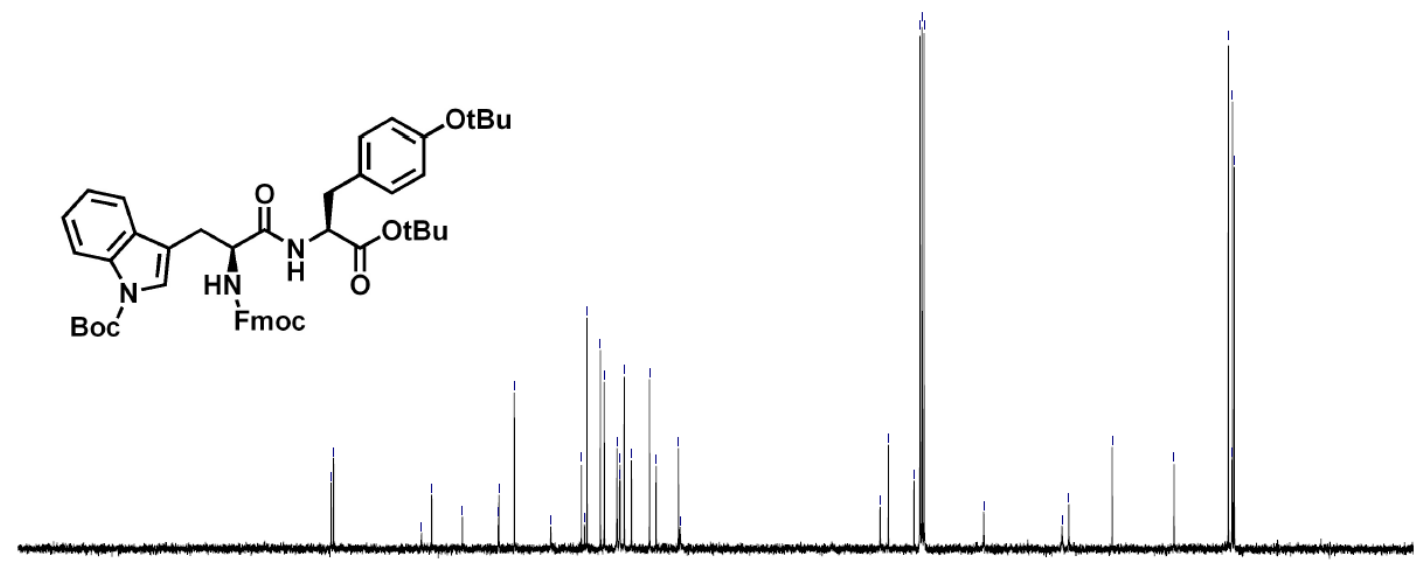

$\begin{array}{lllllllllllllllllllllllllllll} & 210 & 200 & 190 & 180 & 170 & 160 & 150 & 140 & 130 & 120 & 110 & 100 & 90 & 80 & 70 & 60 & 50 & 40 & 30 & 20 & 10 & 0\end{array}$ 


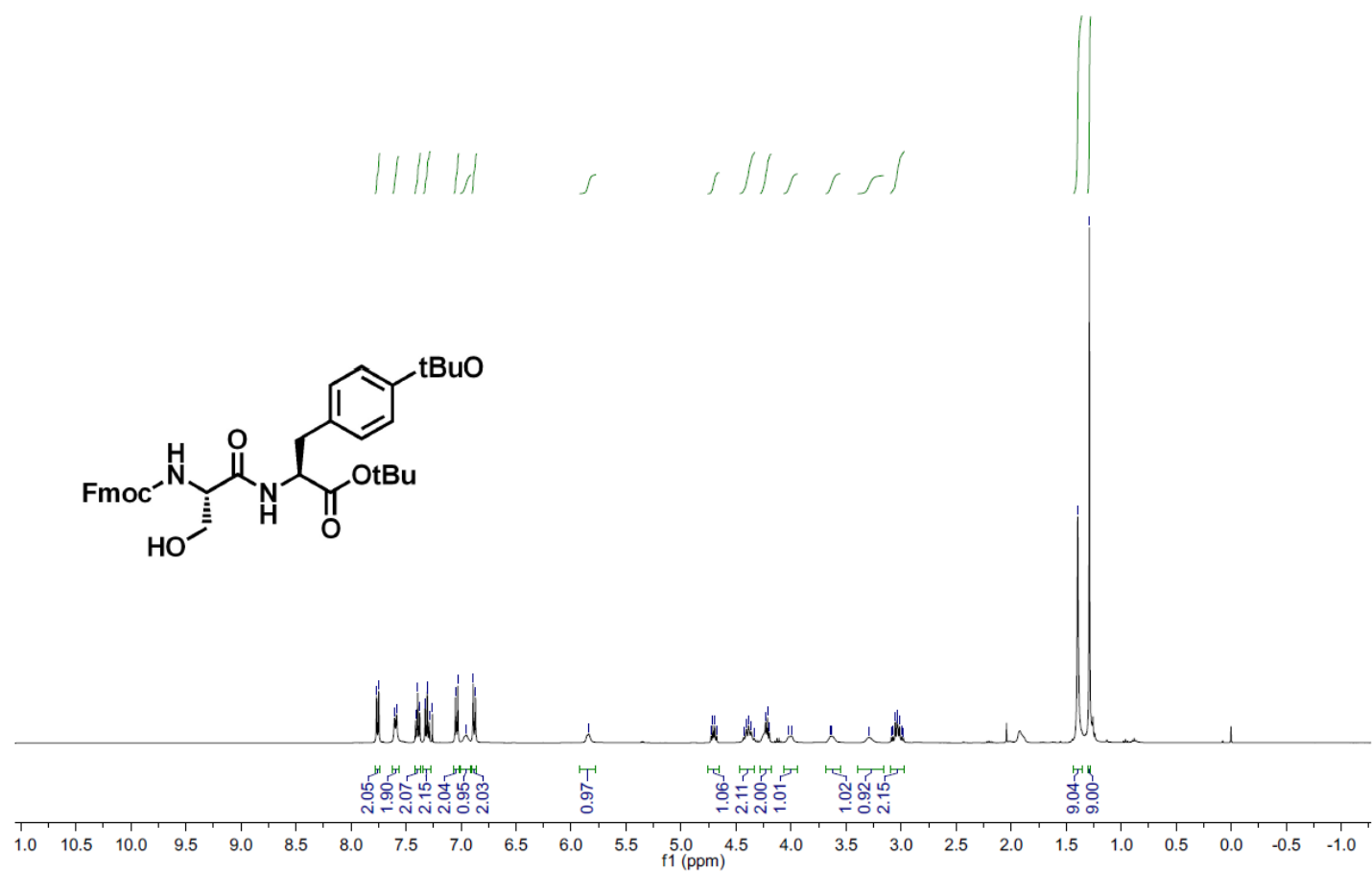

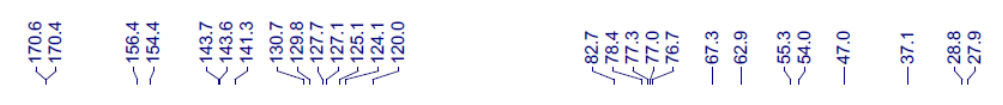

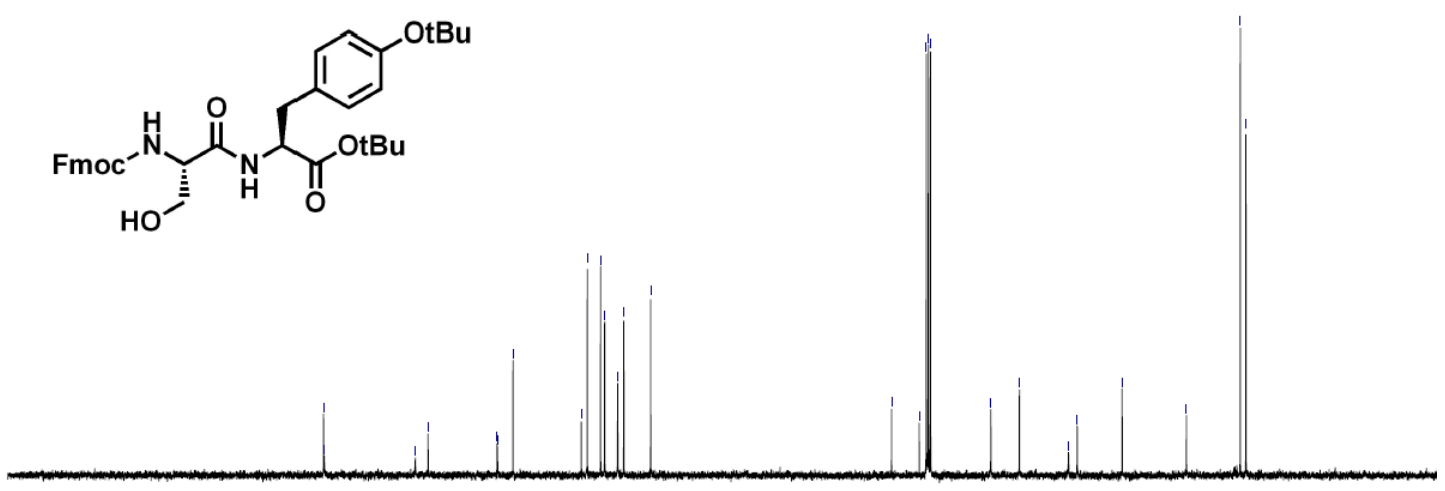

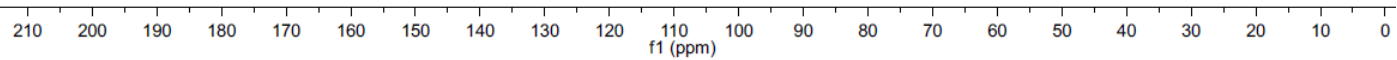




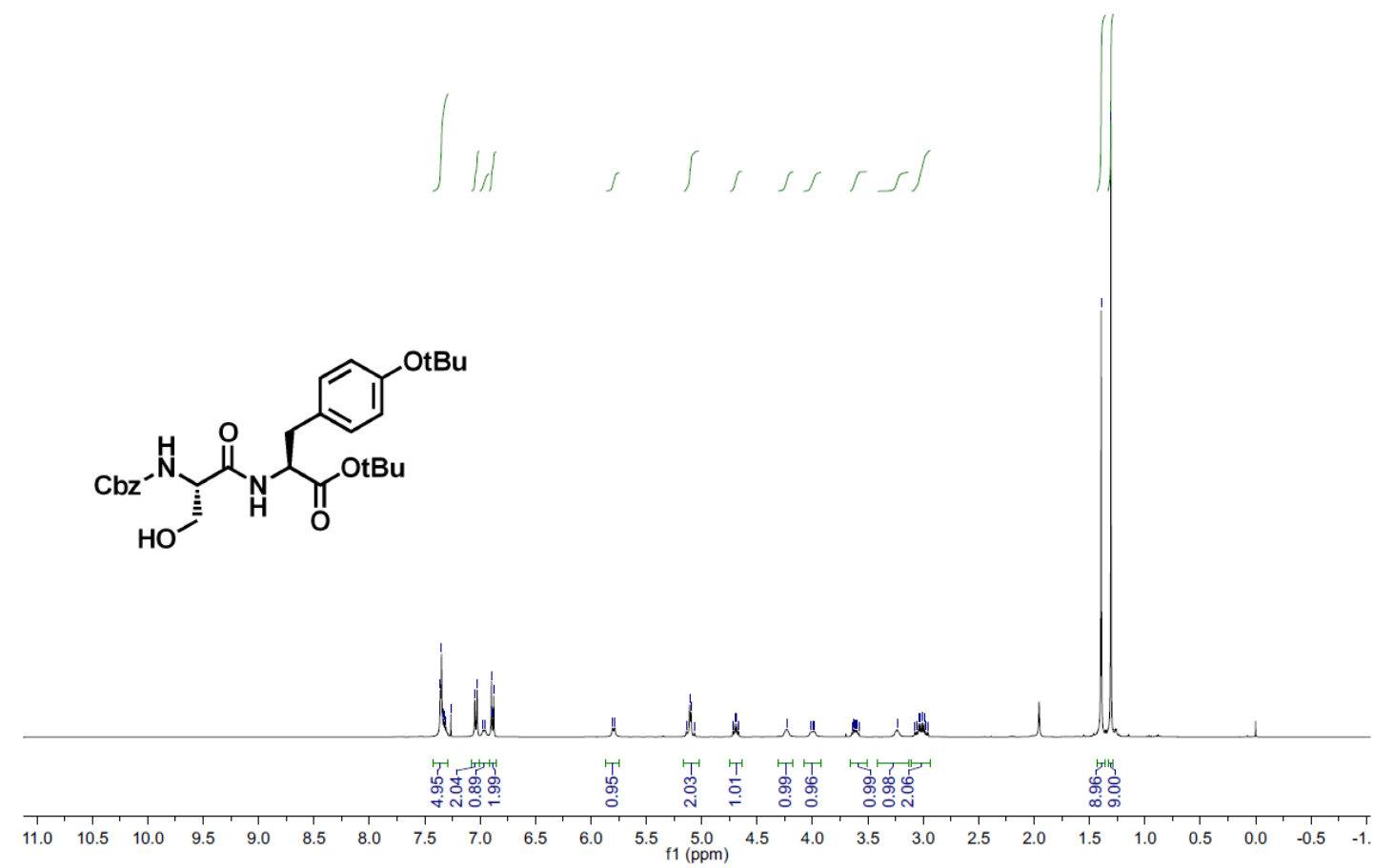

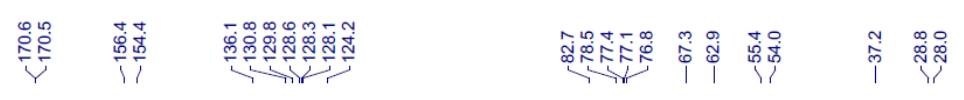

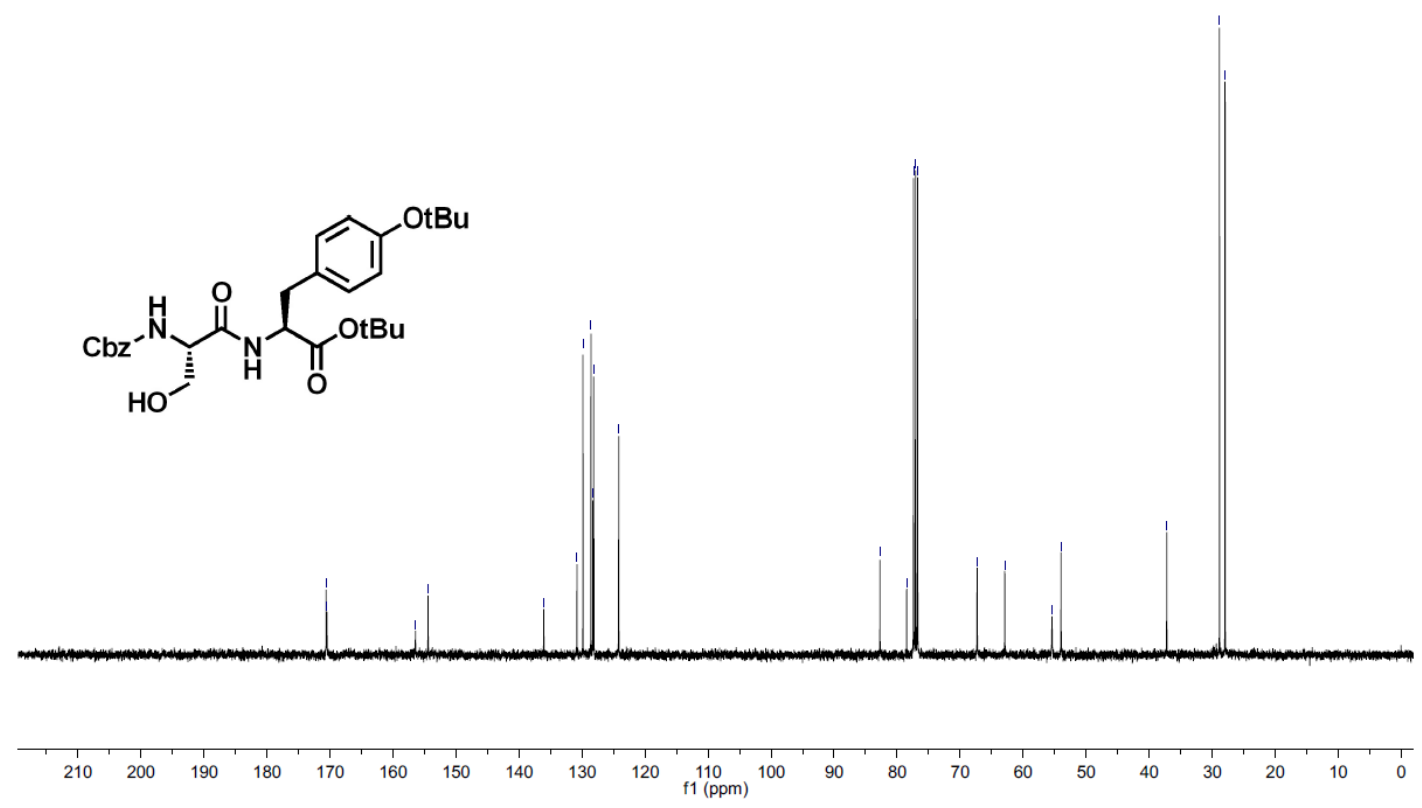



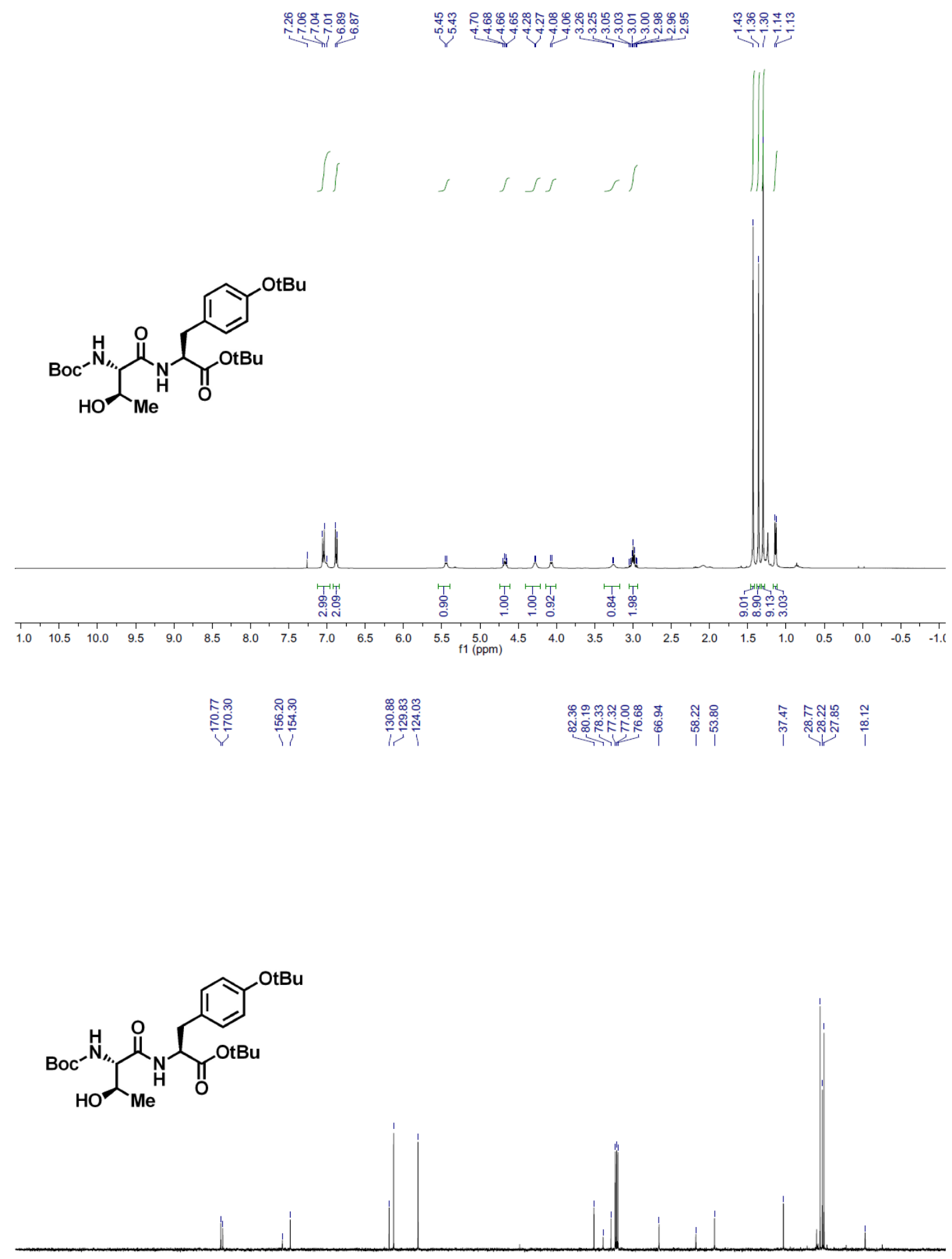

$\begin{array}{lllllllllllllllllllllllllll} & 1 \\ 210 & 200 & 190 & 180 & 170 & 160 & 150 & 140 & 130 & 120 & 110 & 100 & 90 & 80 & 70 & 60 & 50 & 40 & 30 & 20 & 10 & 0\end{array}$ 


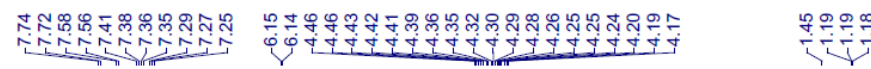
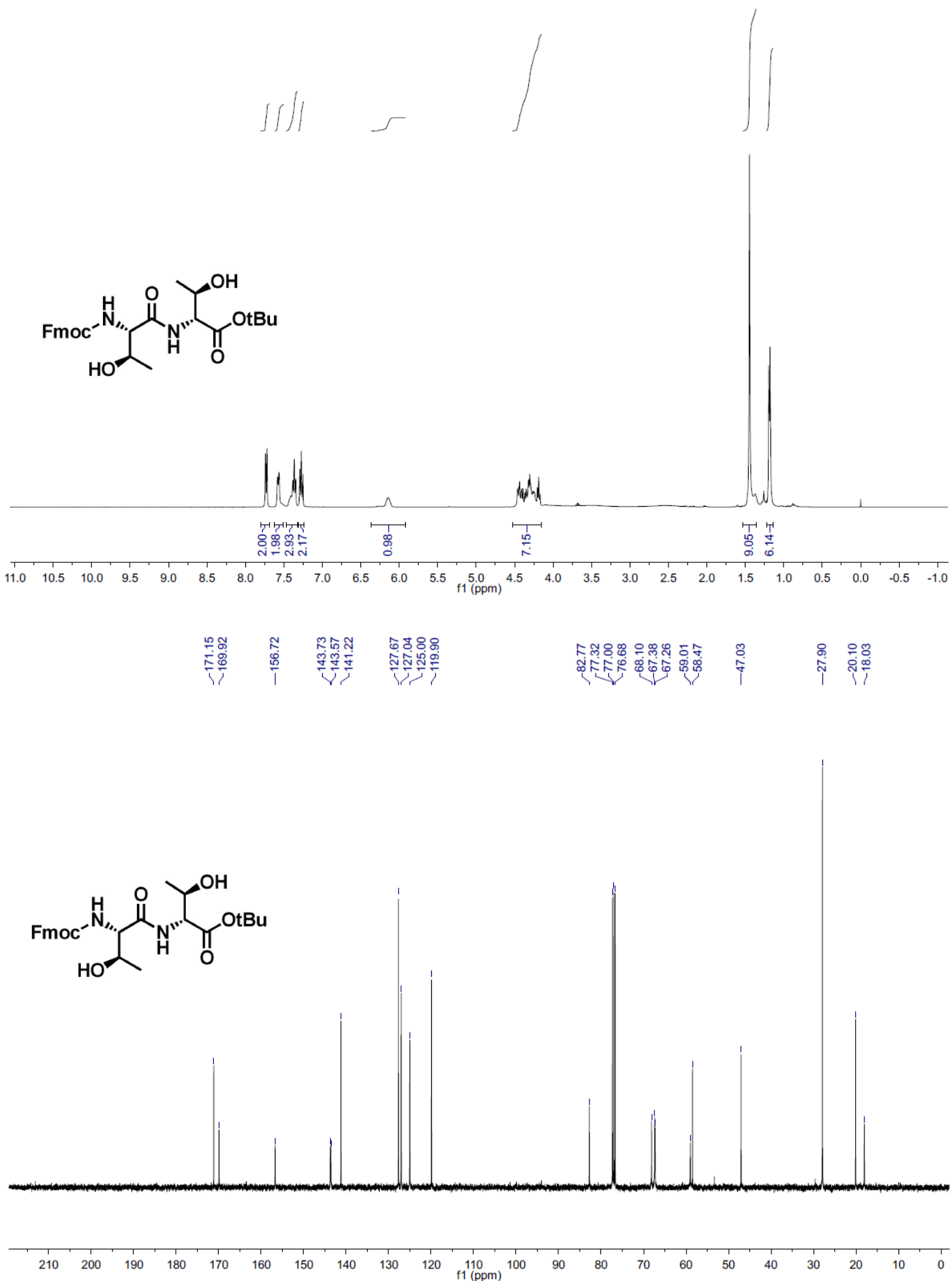

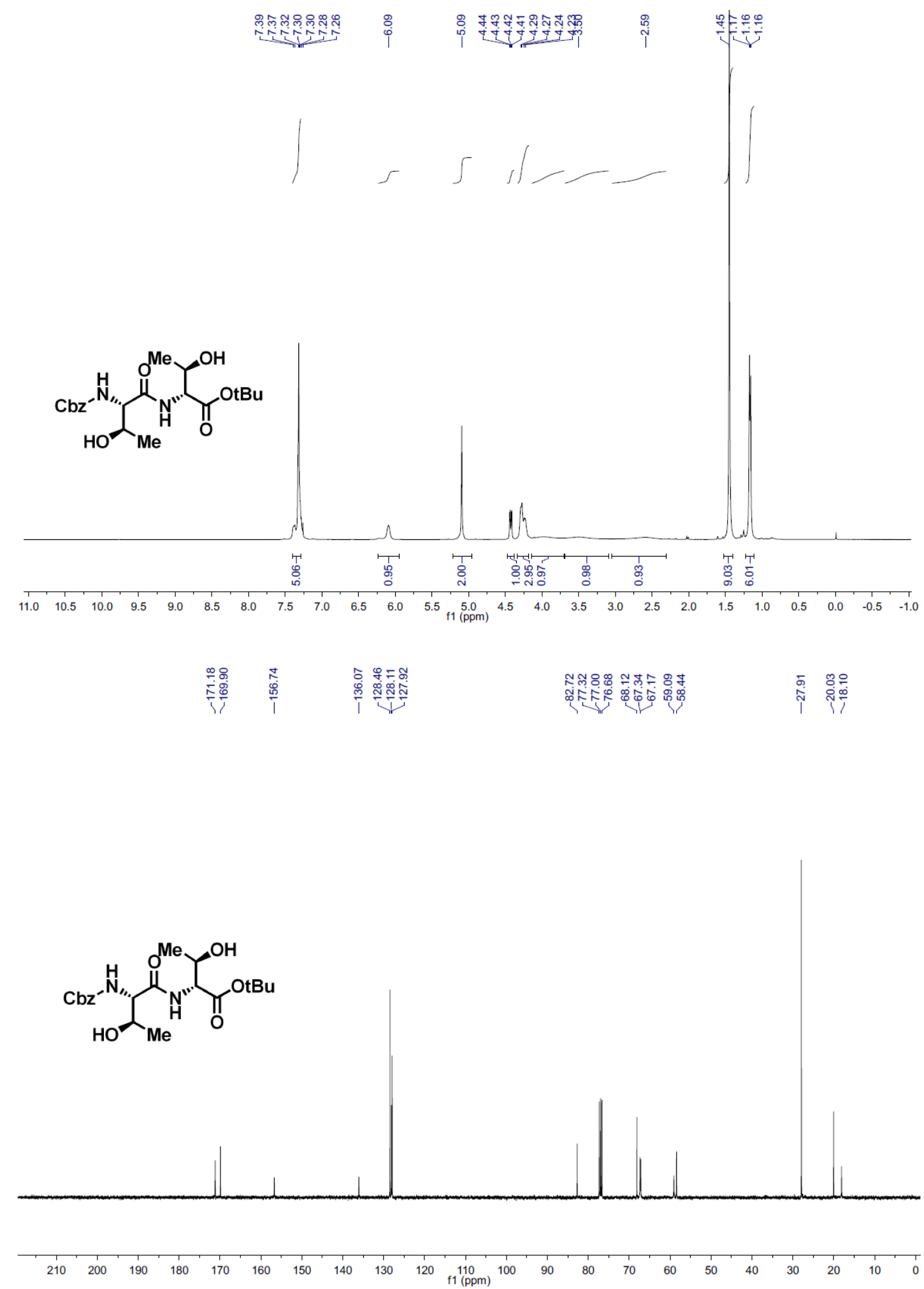


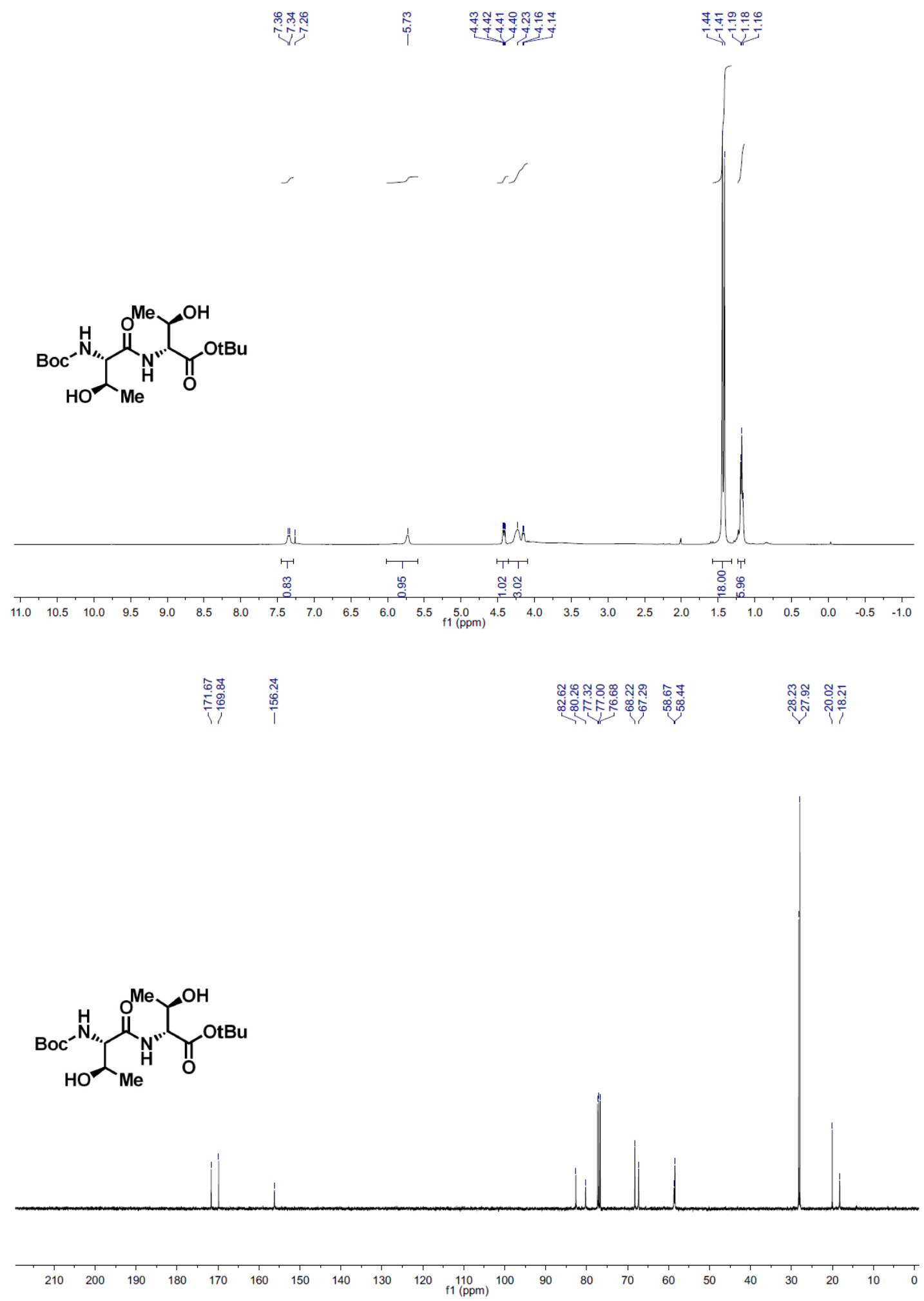



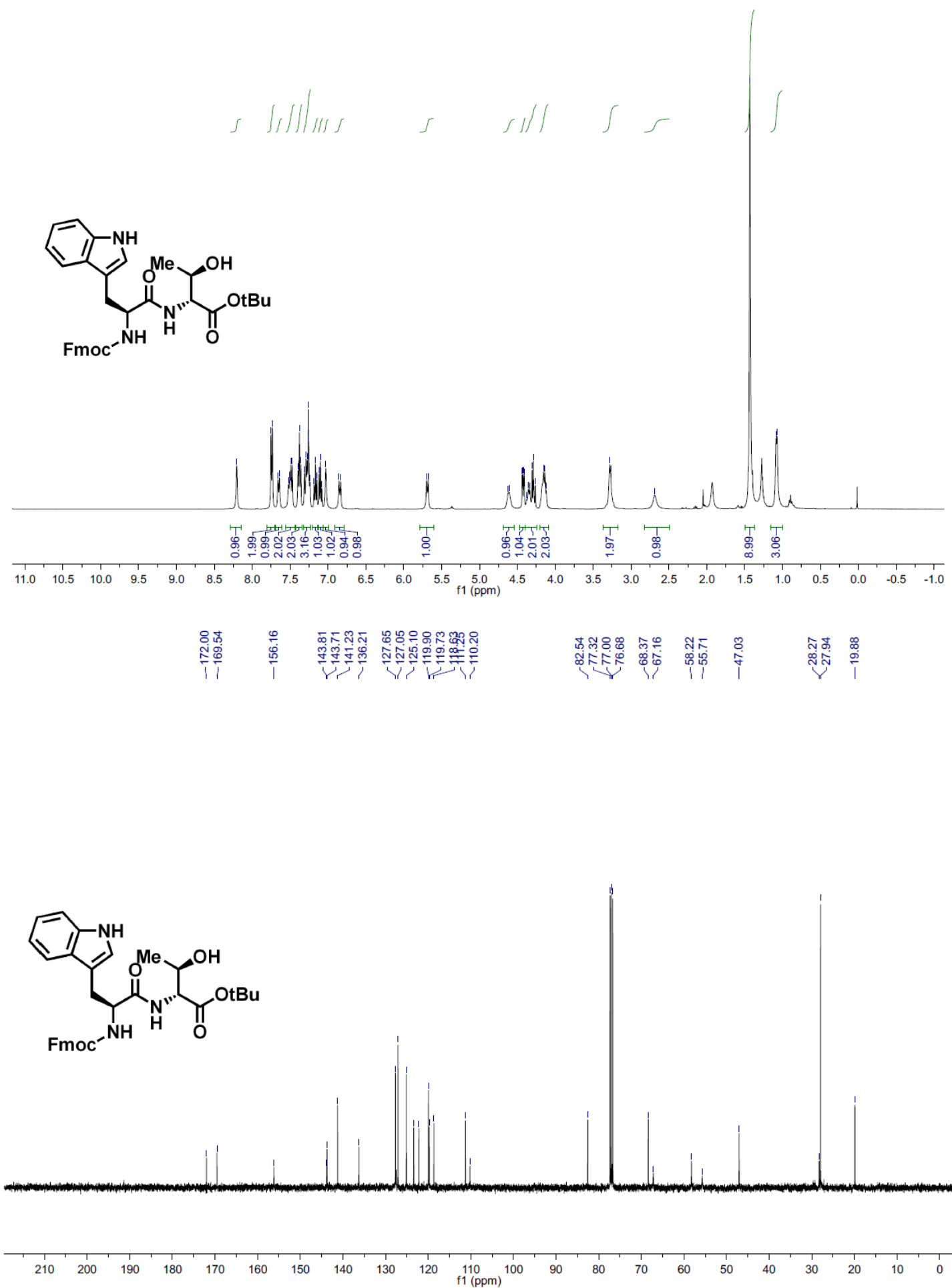


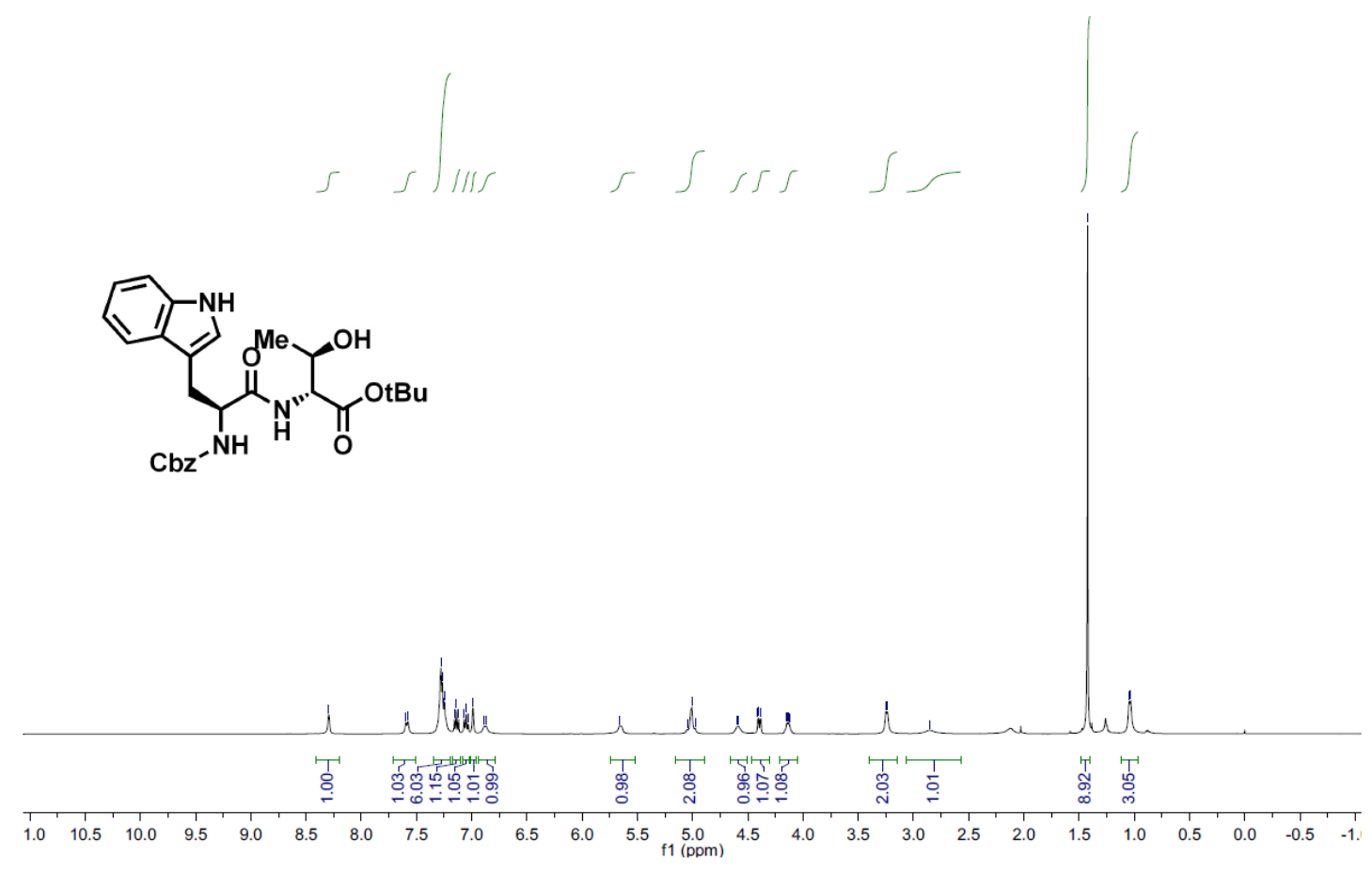

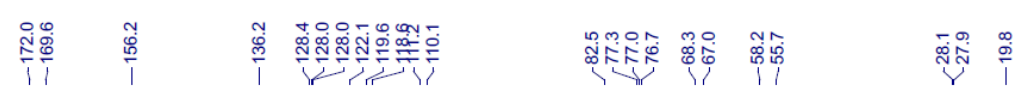

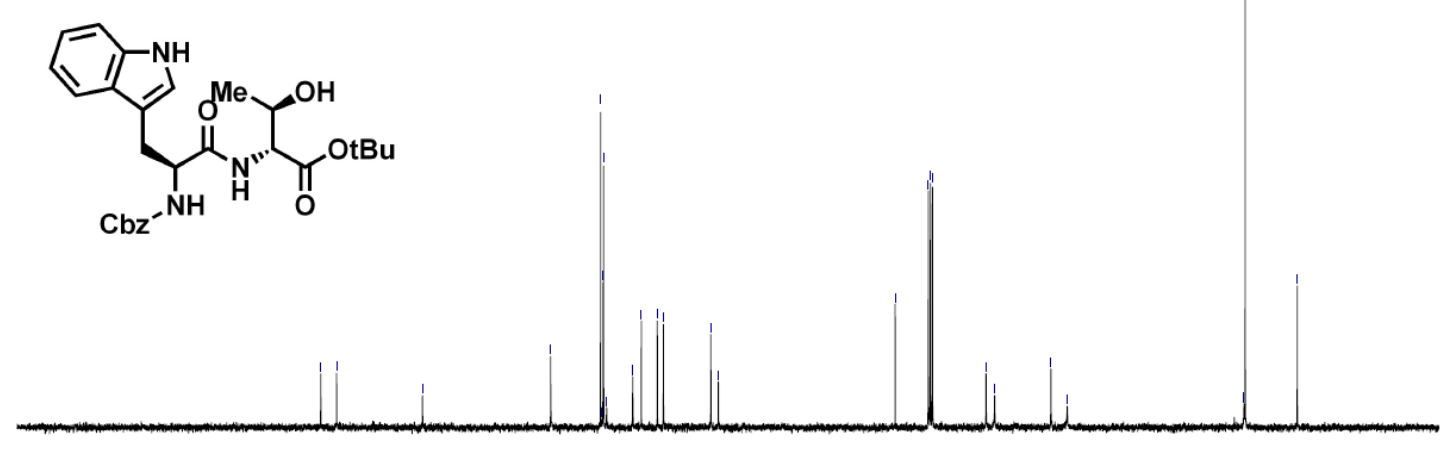

$\begin{array}{llllllllllllllllllllllllll} & 1 \\ 210 & 200 & 190 & 180 & 170 & 160 & 150 & 140 & 130 & 120 & 110 & 100 & 90 & 80 & 70 & 60 & 50 & 40 & 30 & 20 & 10 & 0\end{array}$ 


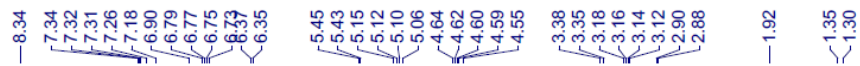

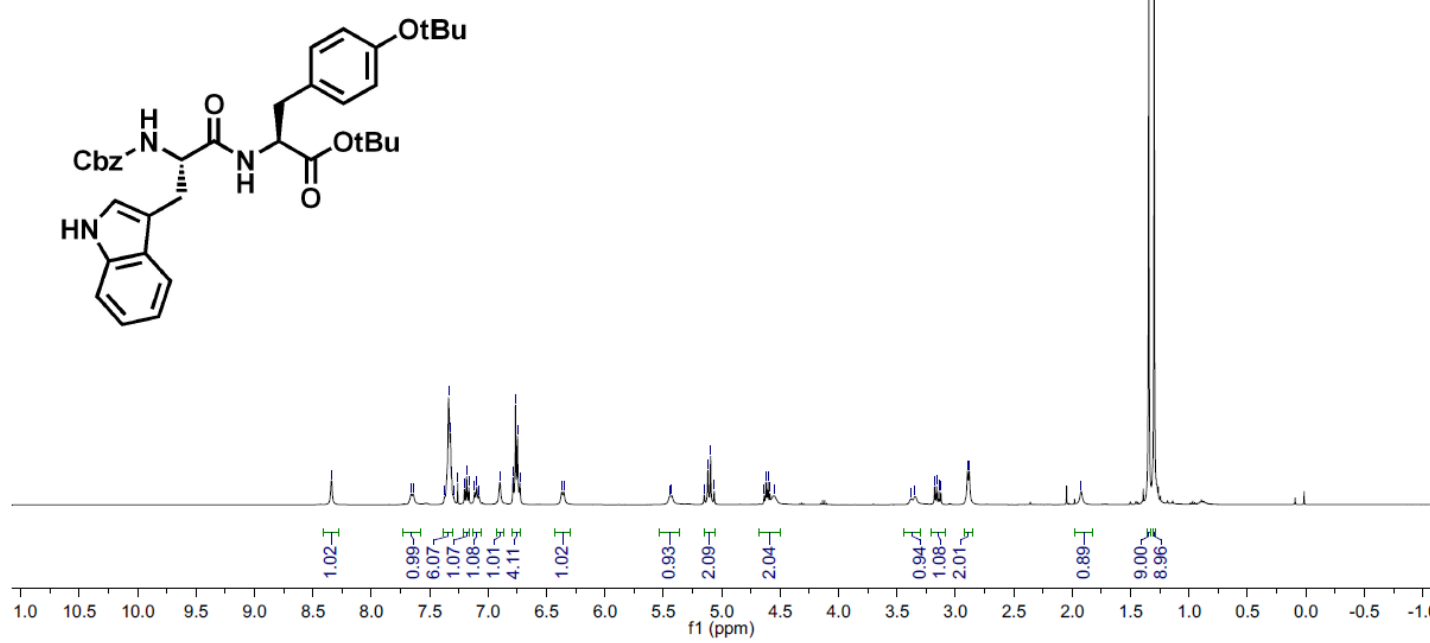

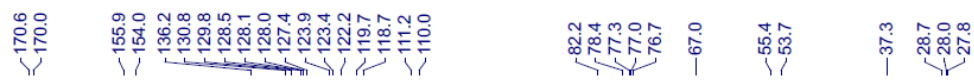

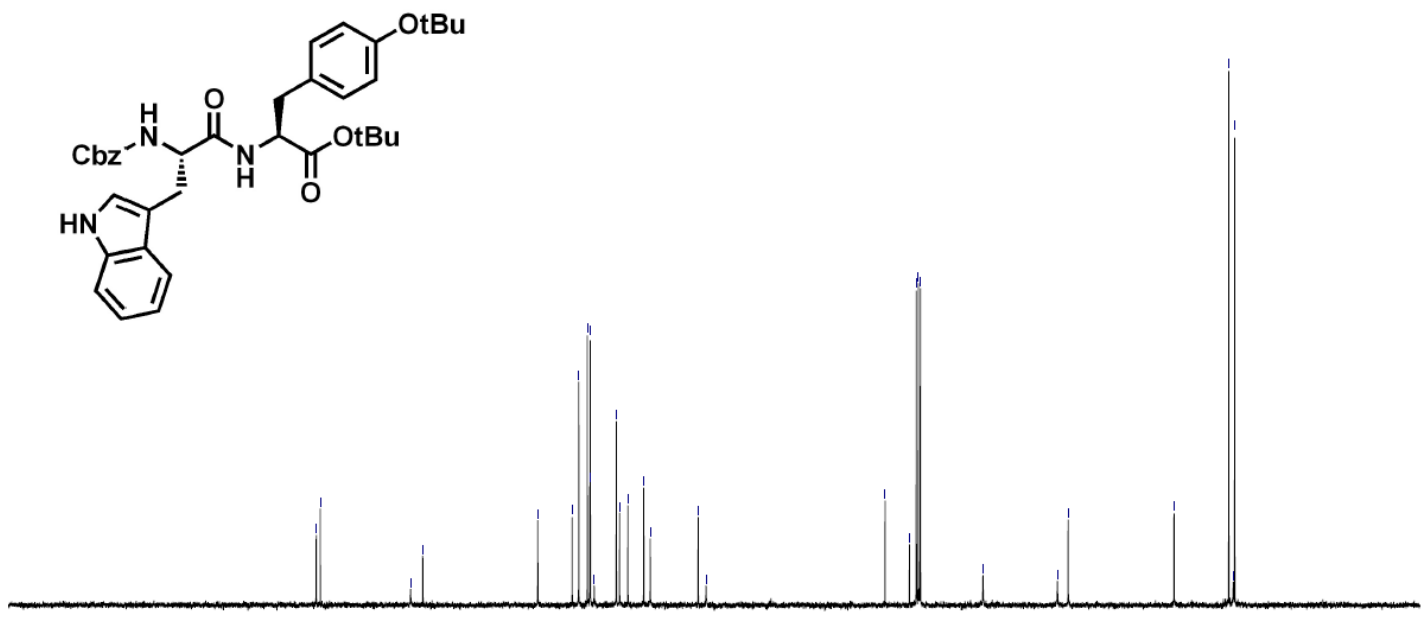

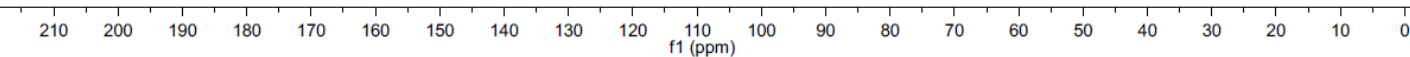




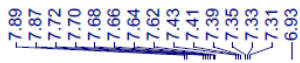

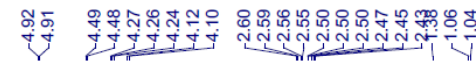

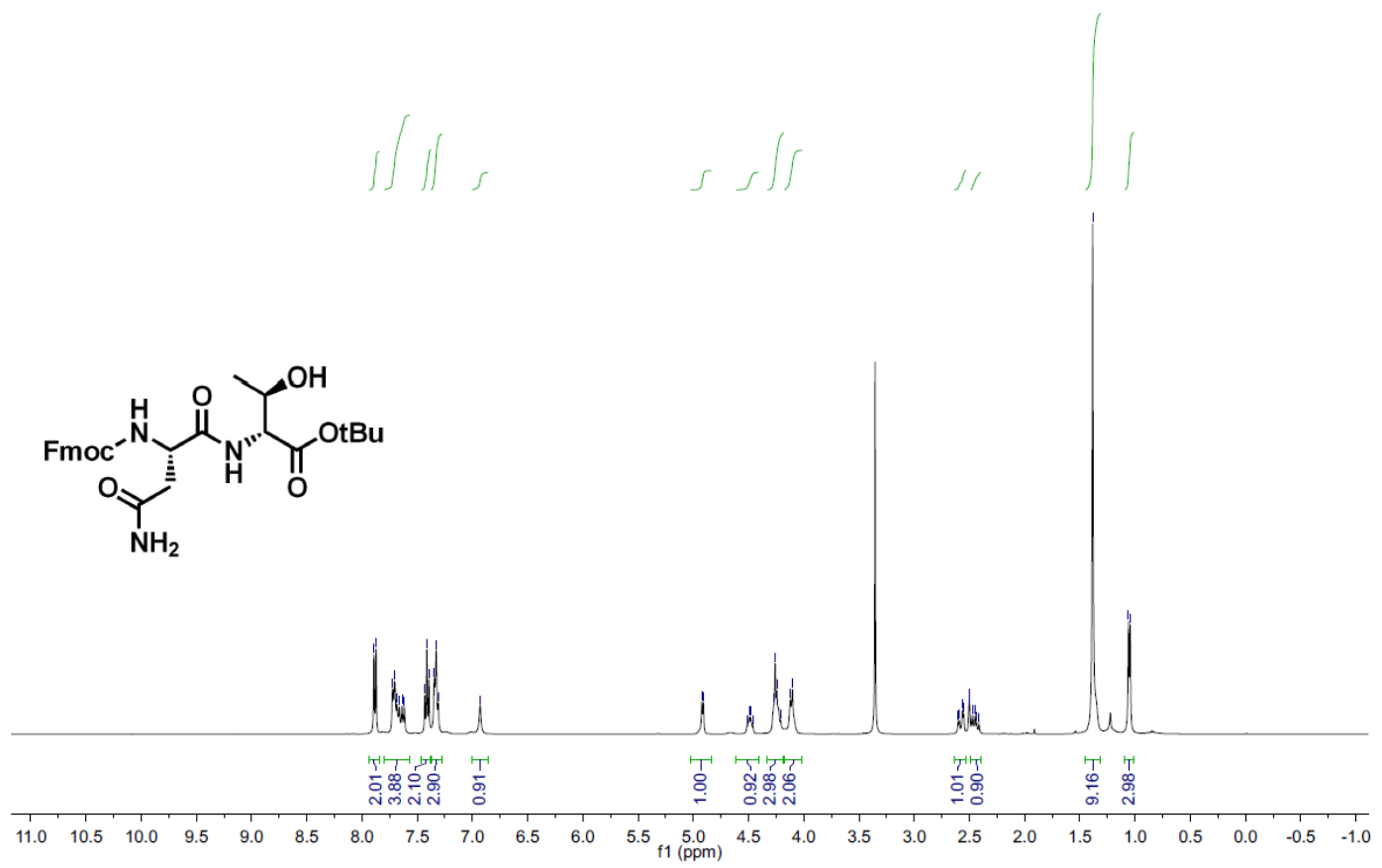

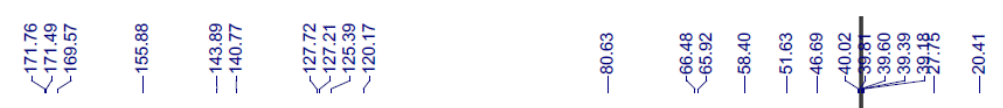

$\underbrace{\mathrm{O}}_{\mathrm{NH}_{2}}$

$\begin{array}{lllllllllllllllllllllllll}1 & 1 & 200 & 190 & 180 & 170 & 160 & 150 & 140 & 130 & 120 & \underset{f 1}{110} & 100 & 90 & 80 & 70 & 60 & 50 & 40 & 30 & 20 & 10 & 0\end{array}$ 

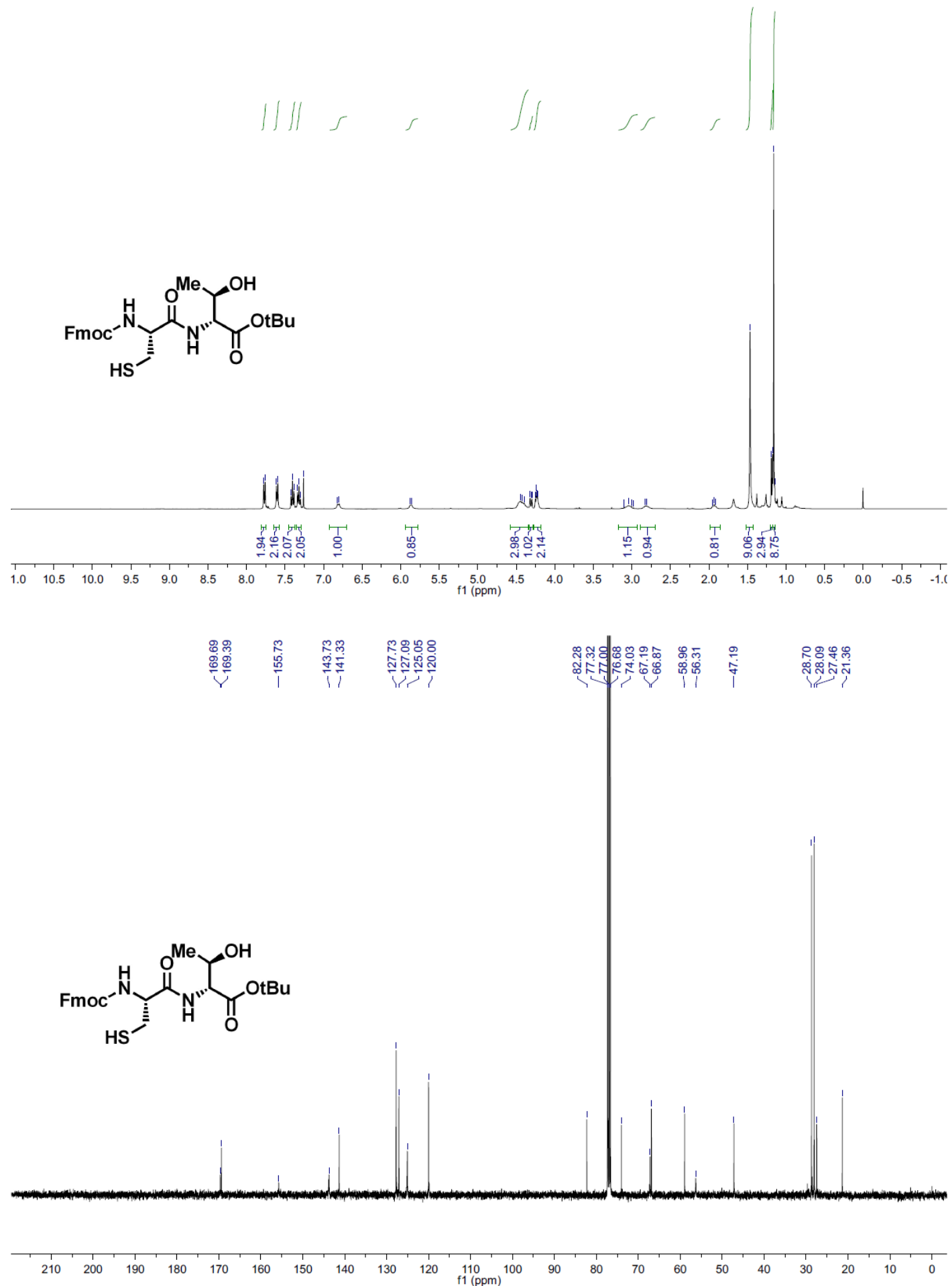


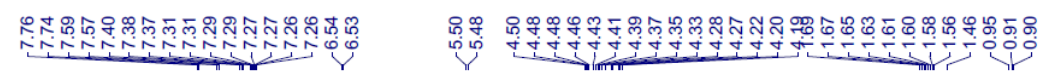
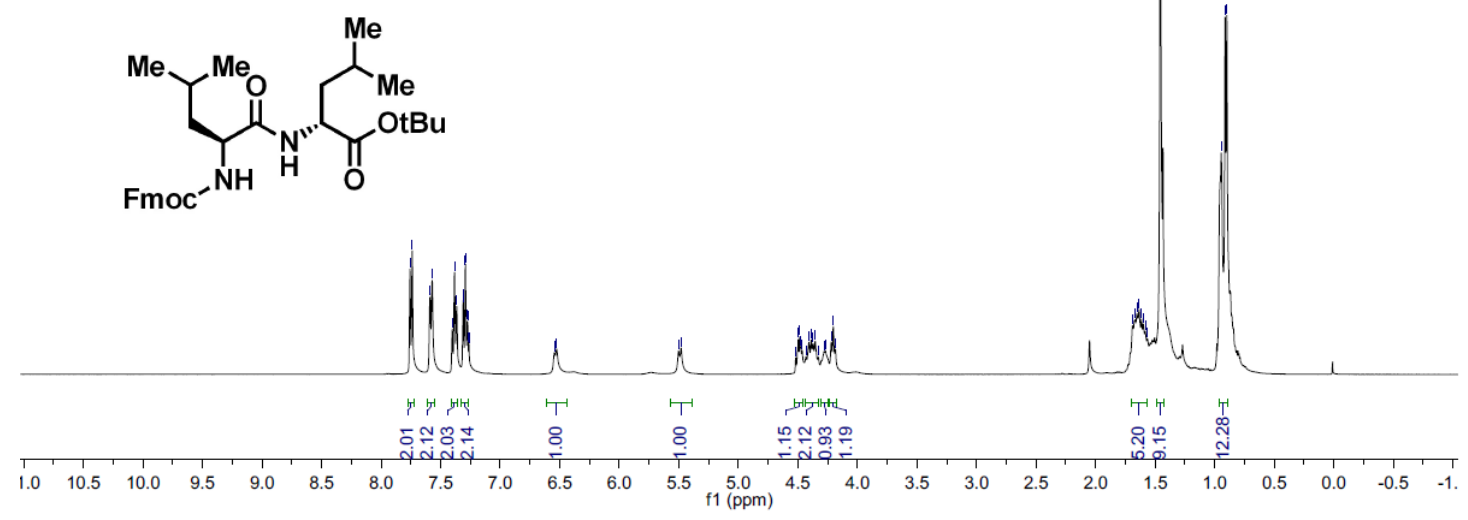

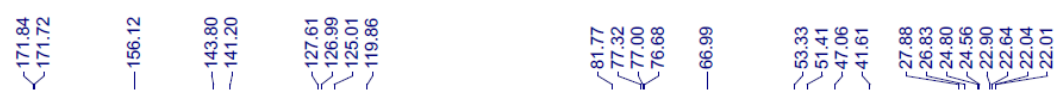
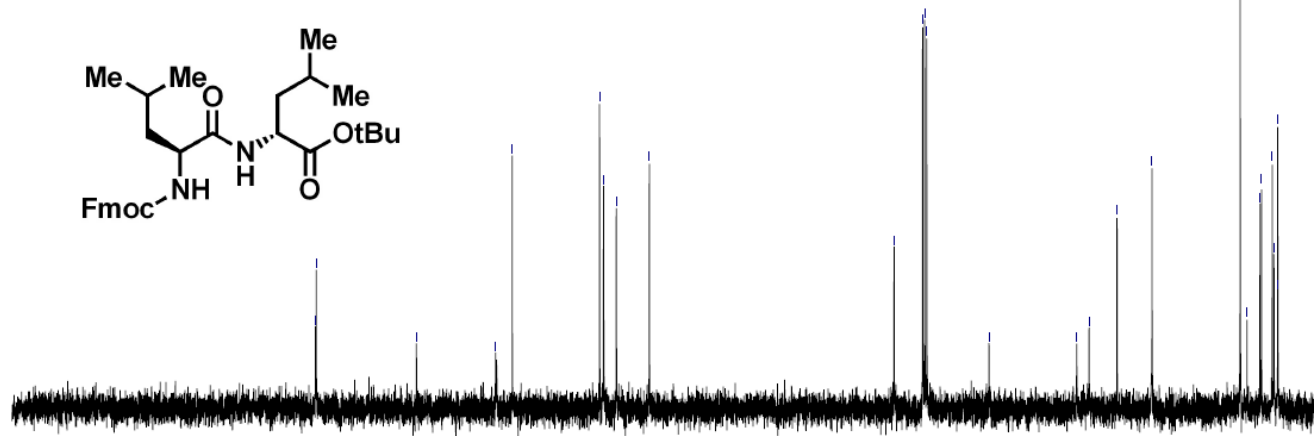

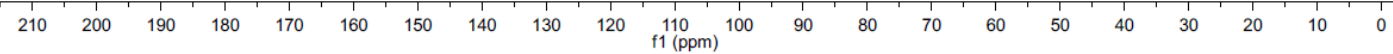




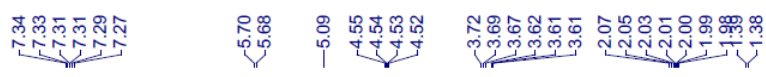

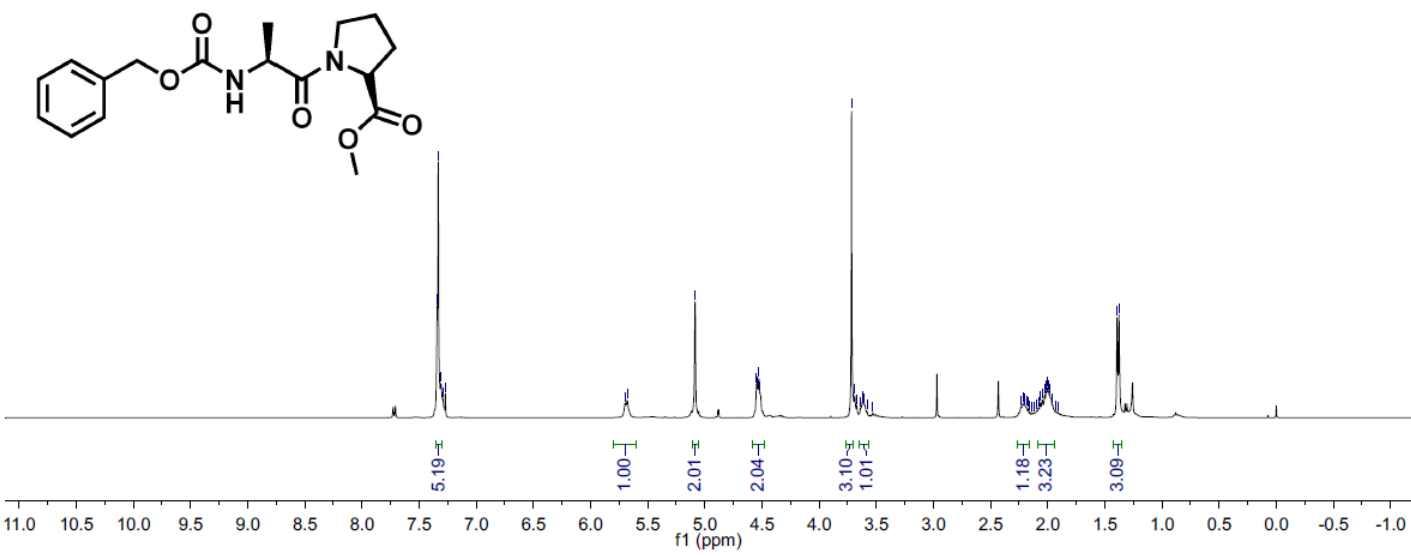

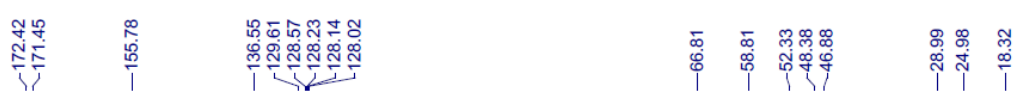

(1)

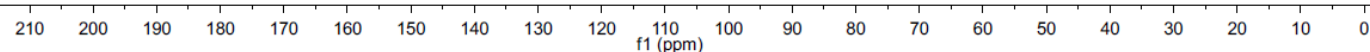


$\mathrm{Fmoc}_{\mathrm{Me}}^{\mathrm{N}} \mathrm{Me}_{\mathrm{Me}}^{\mathrm{O}} \mathrm{N}_{\mathrm{He}}^{\mathrm{OtBu}}$

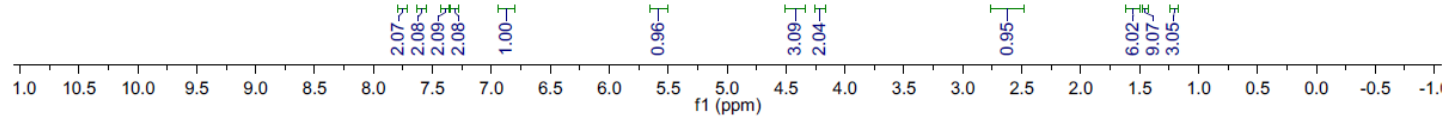
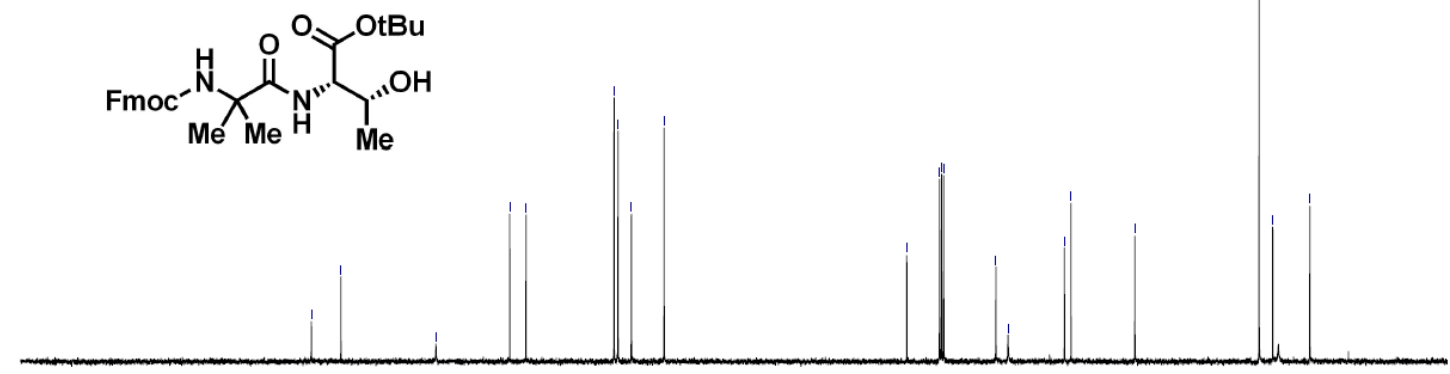

$\begin{array}{llllllllllllllllllllllllllll}210 & 200 & 190 & 180 & 170 & 160 & 150 & 140 & 130 & 120 & 110 & 100 & 90 & 80 & 70 & 60 & 50 & 40 & 30 & 20 & 10 & 0\end{array}$ 


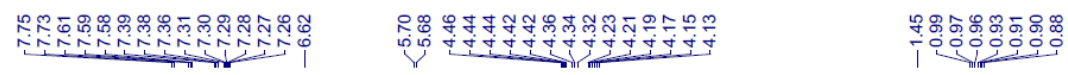
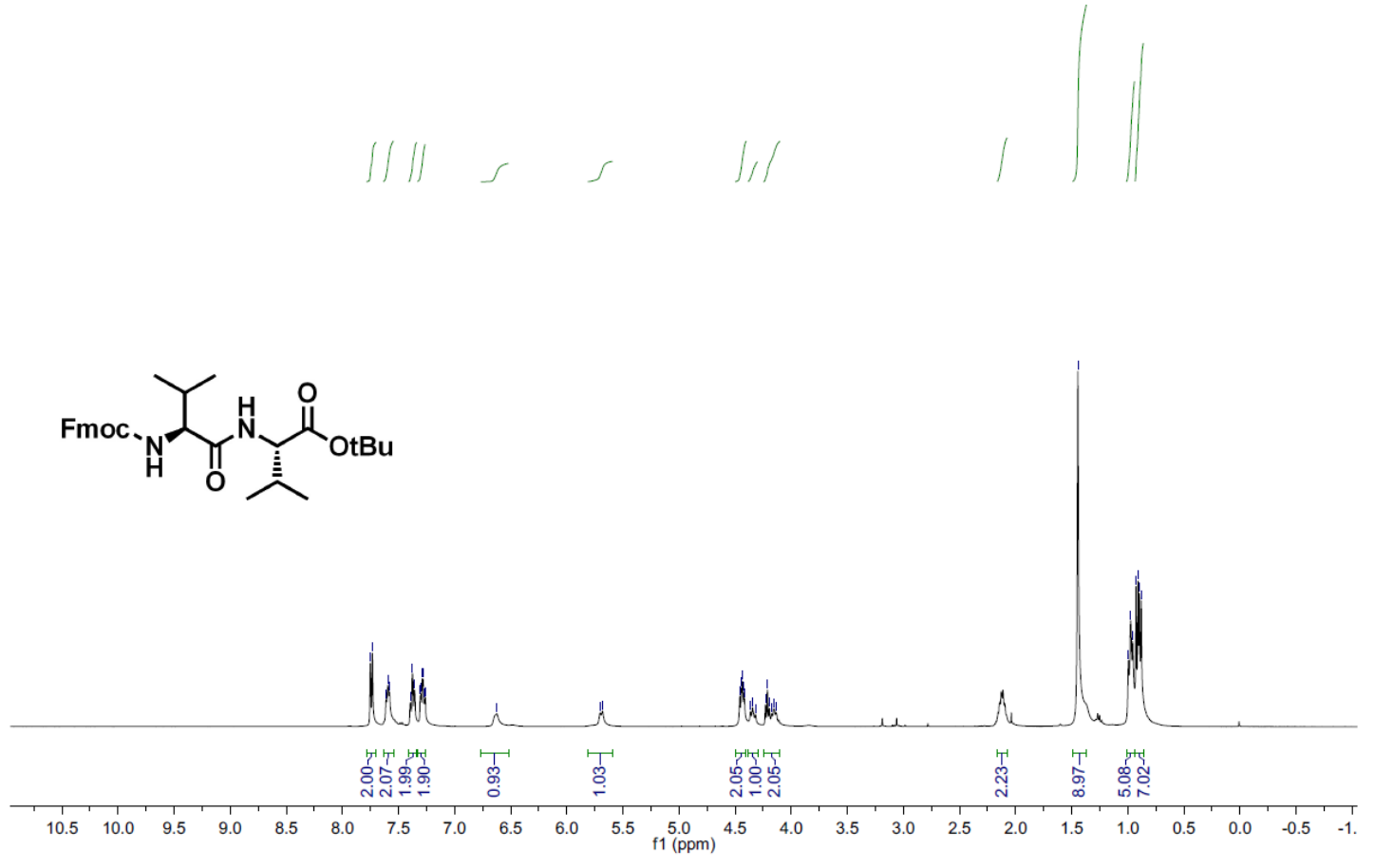

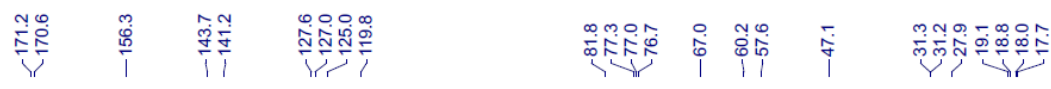

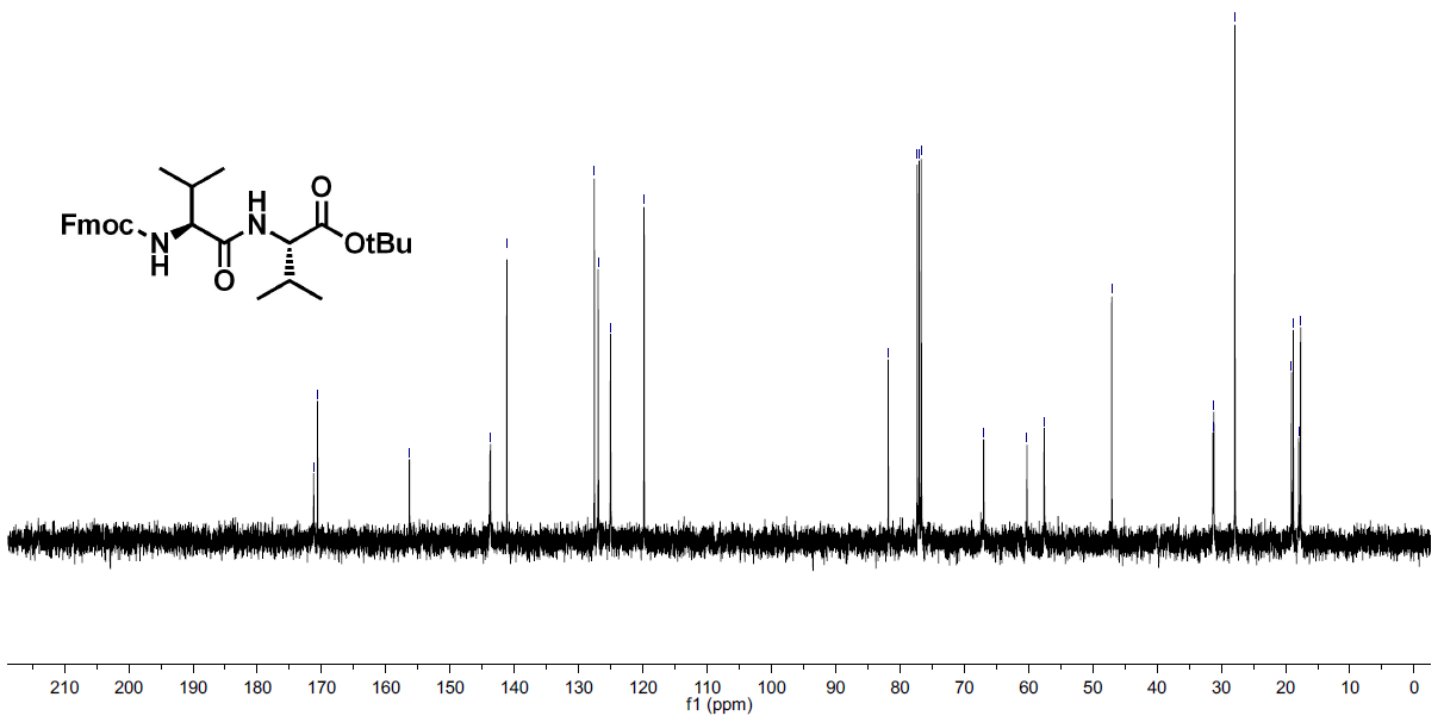




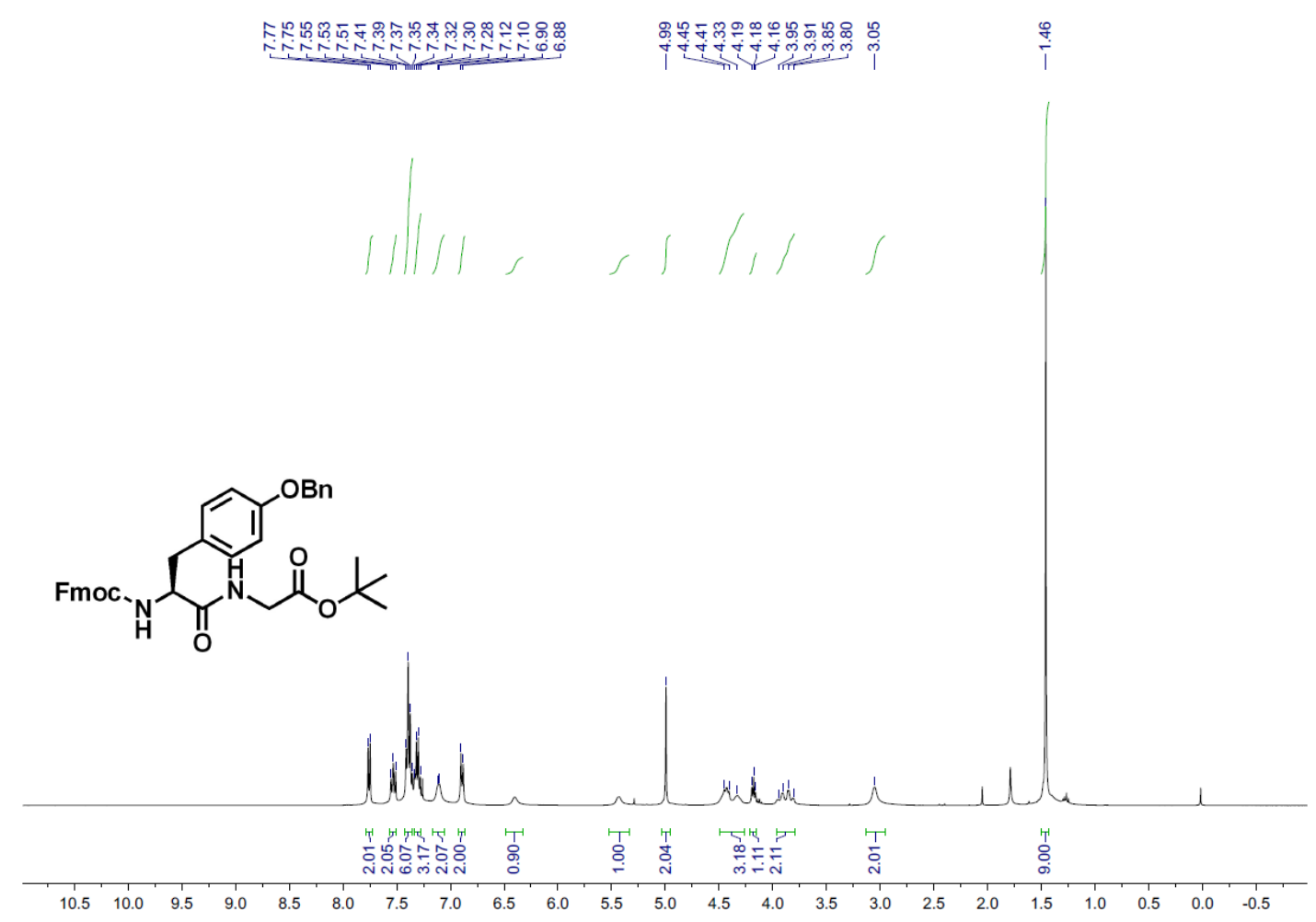

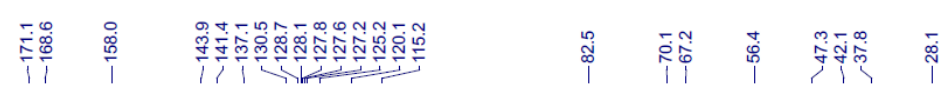

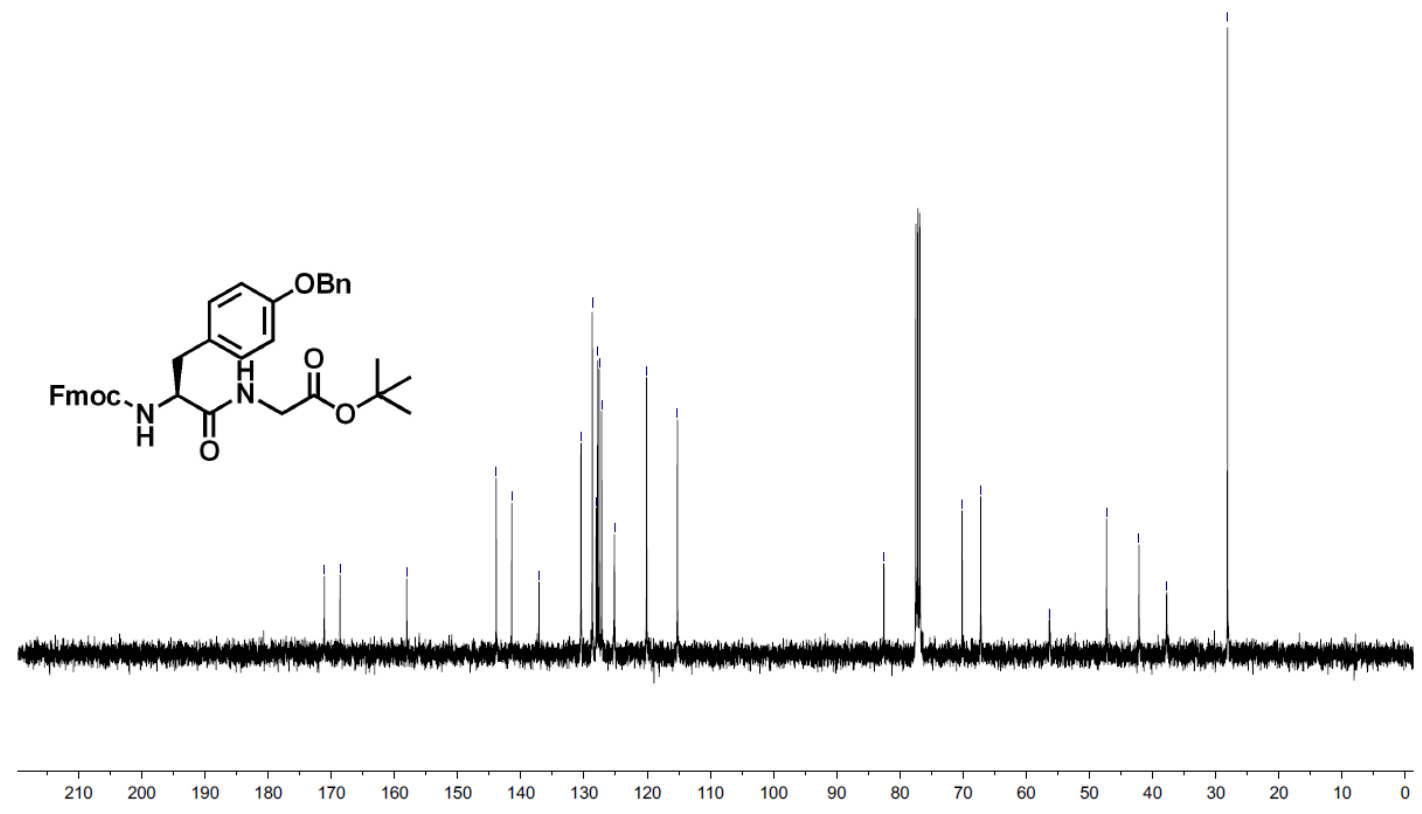




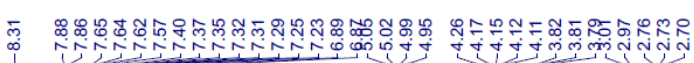

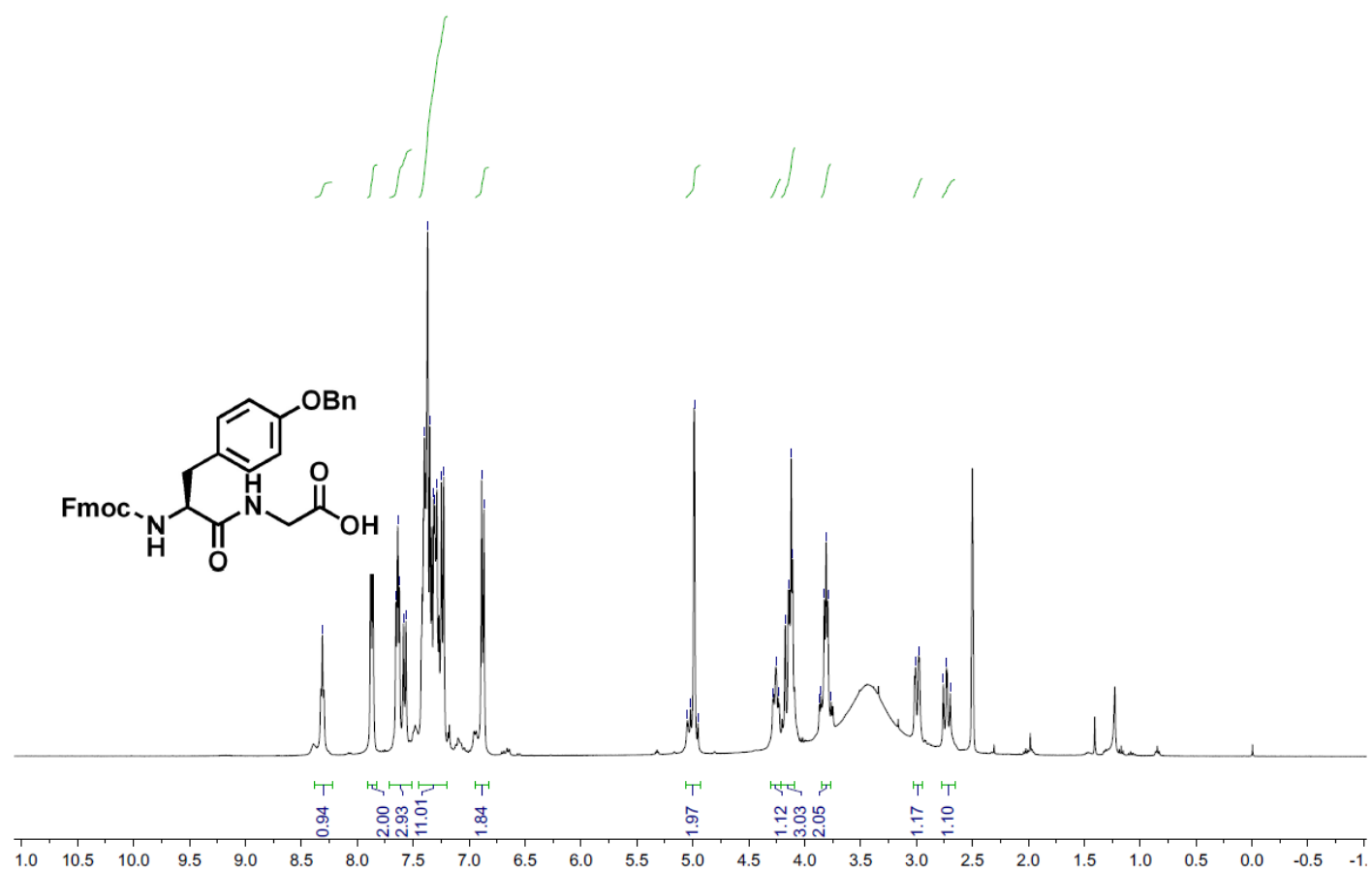

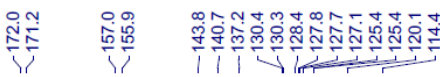

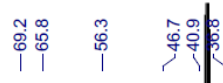
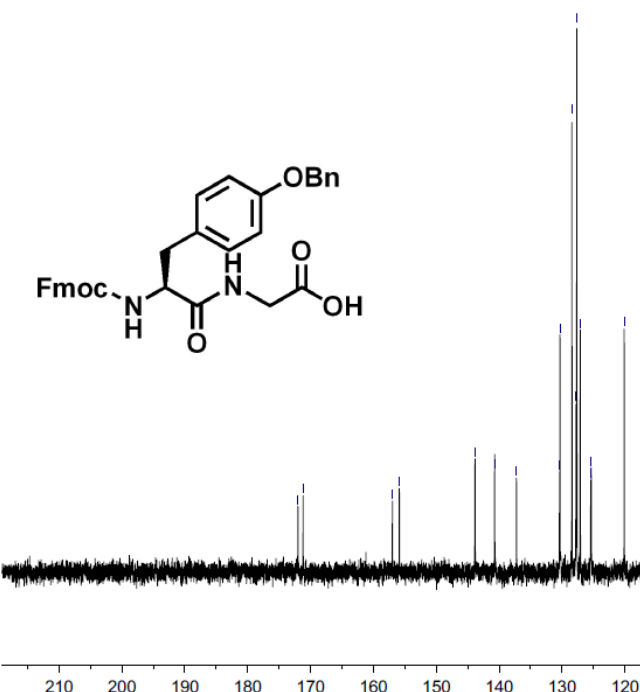

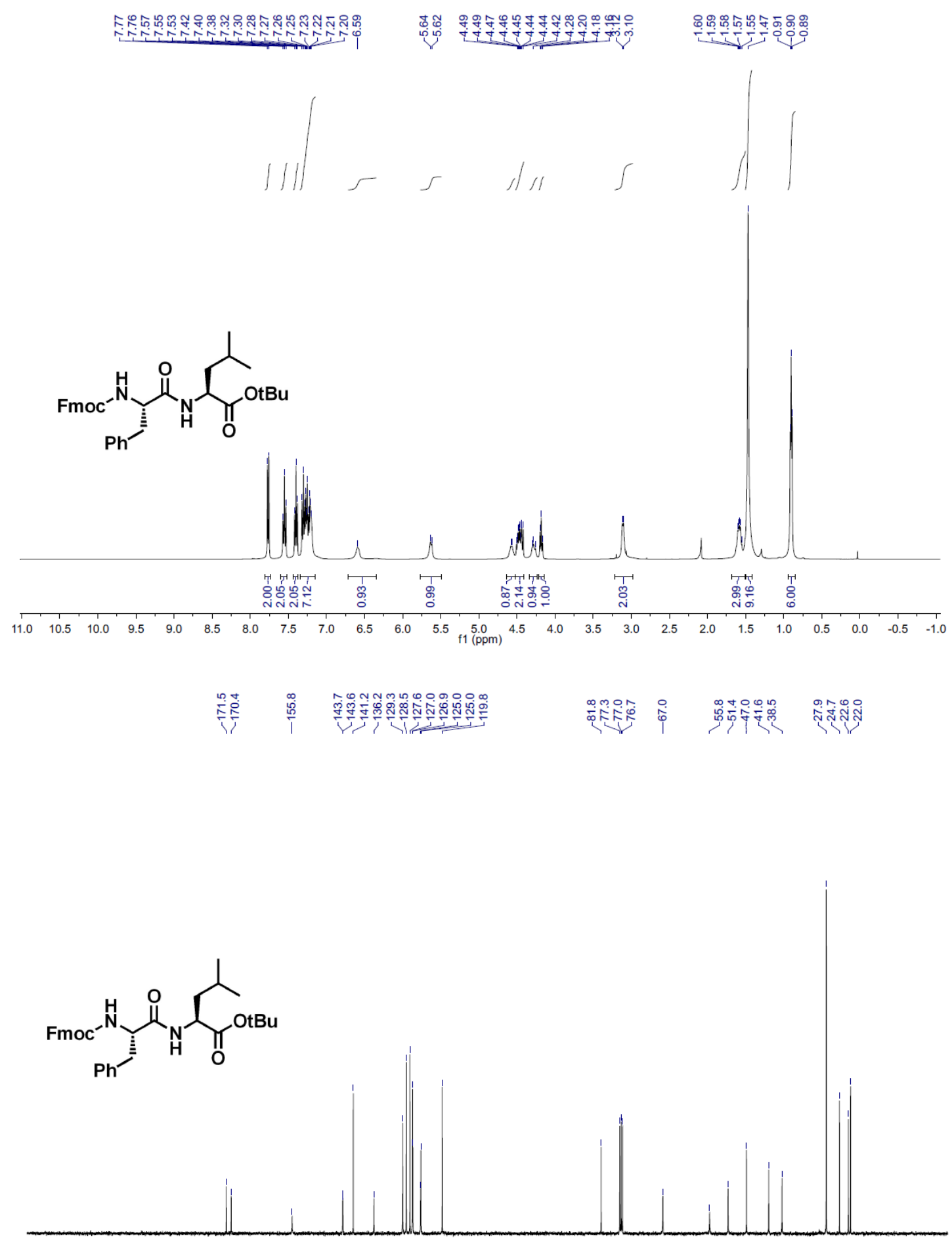

$\begin{array}{lllllllllllllllllllllllllllll}210 & 200 & 190 & 180 & 170 & 160 & 150 & 140 & 130 & 120 & \begin{array}{l}110 \\ \mathrm{f} 1(\mathrm{ppm})\end{array} & 100 & 90 & 80 & 70 & 60 & 50 & 40 & 30 & 20 & 10 & 0\end{array}$ 
K

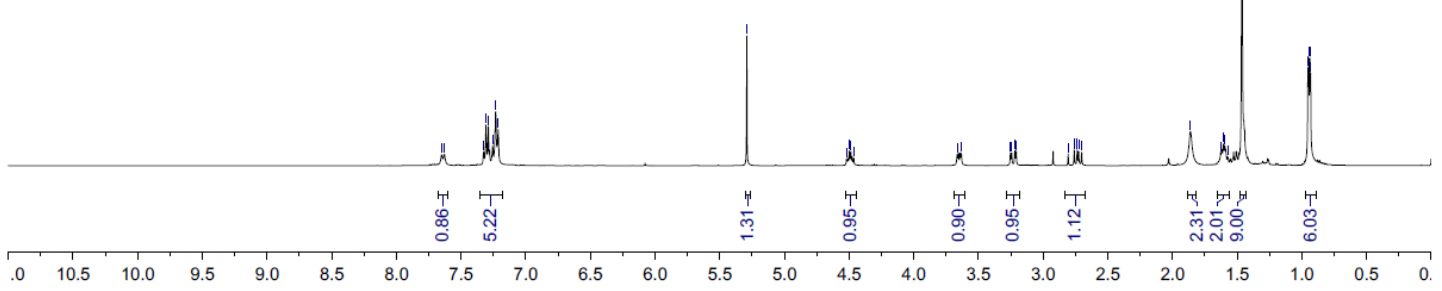

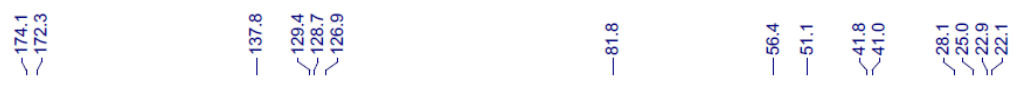
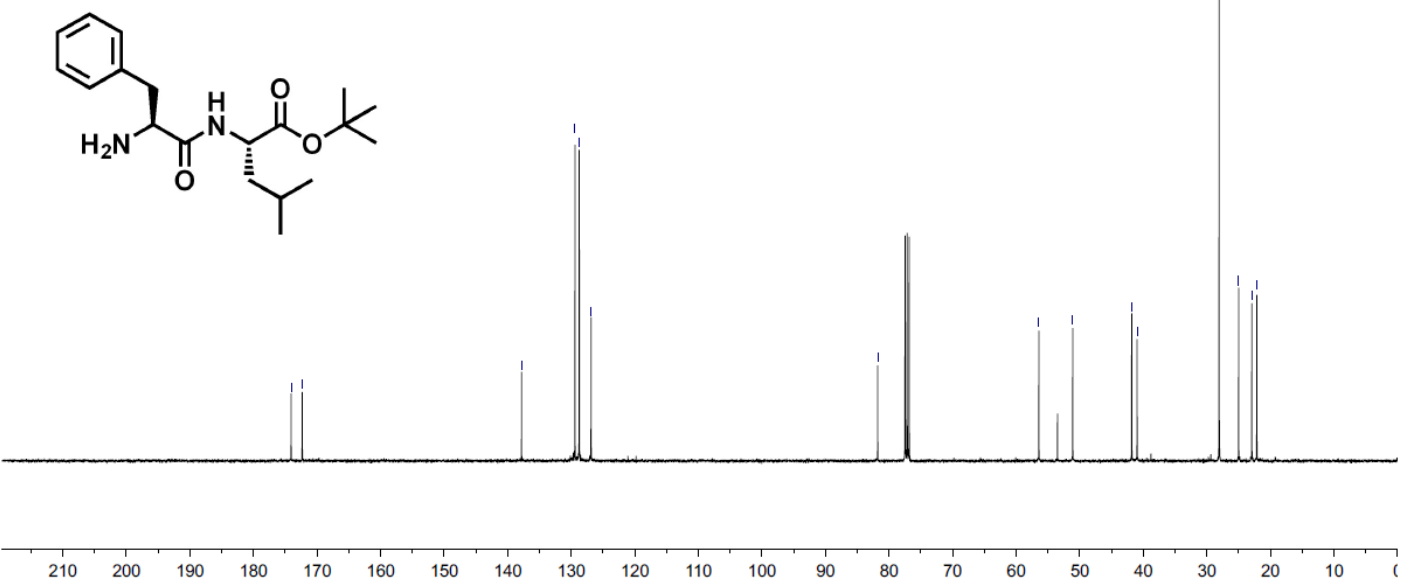
900

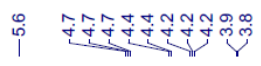

要要

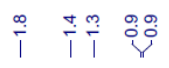
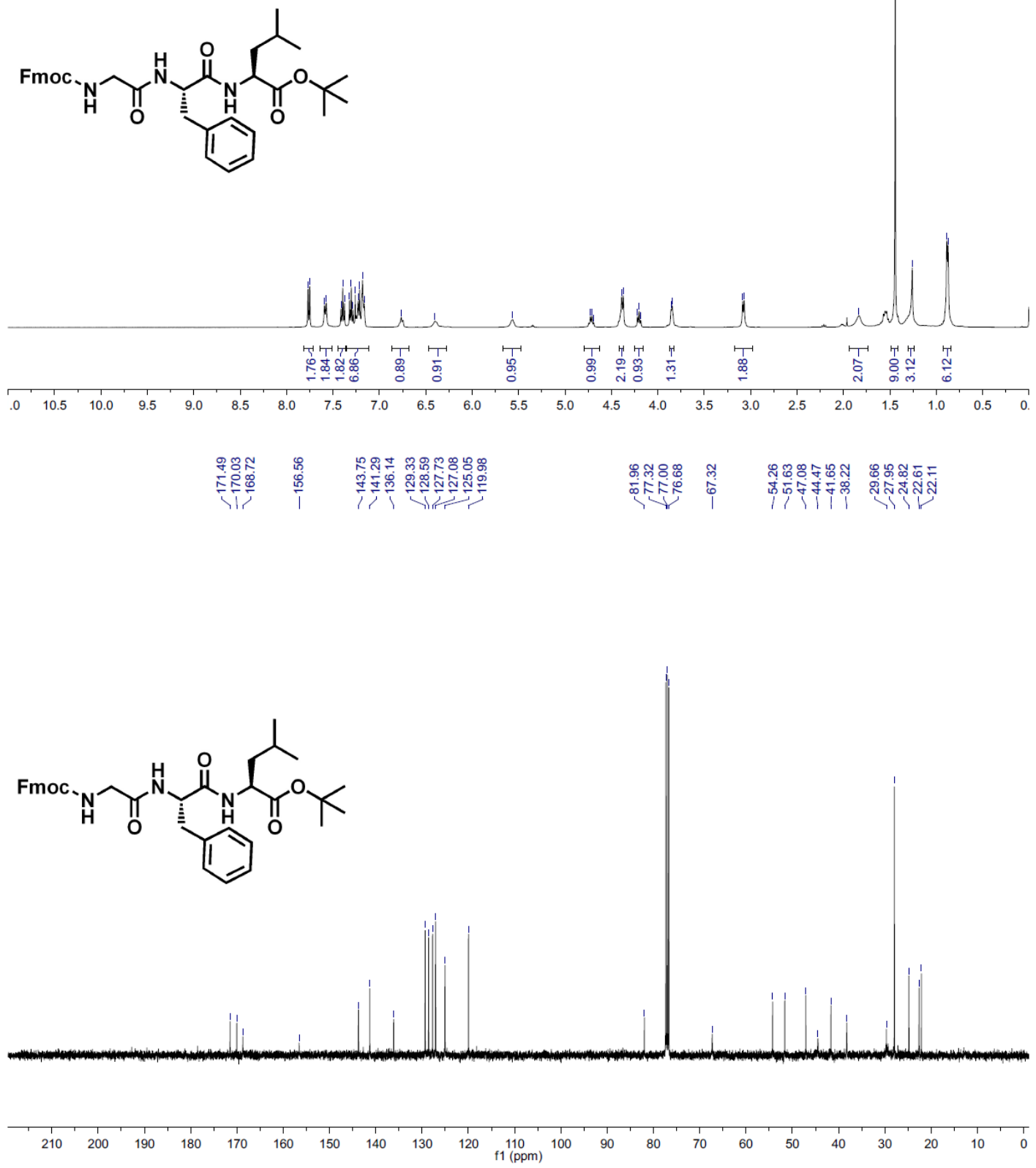

S117 

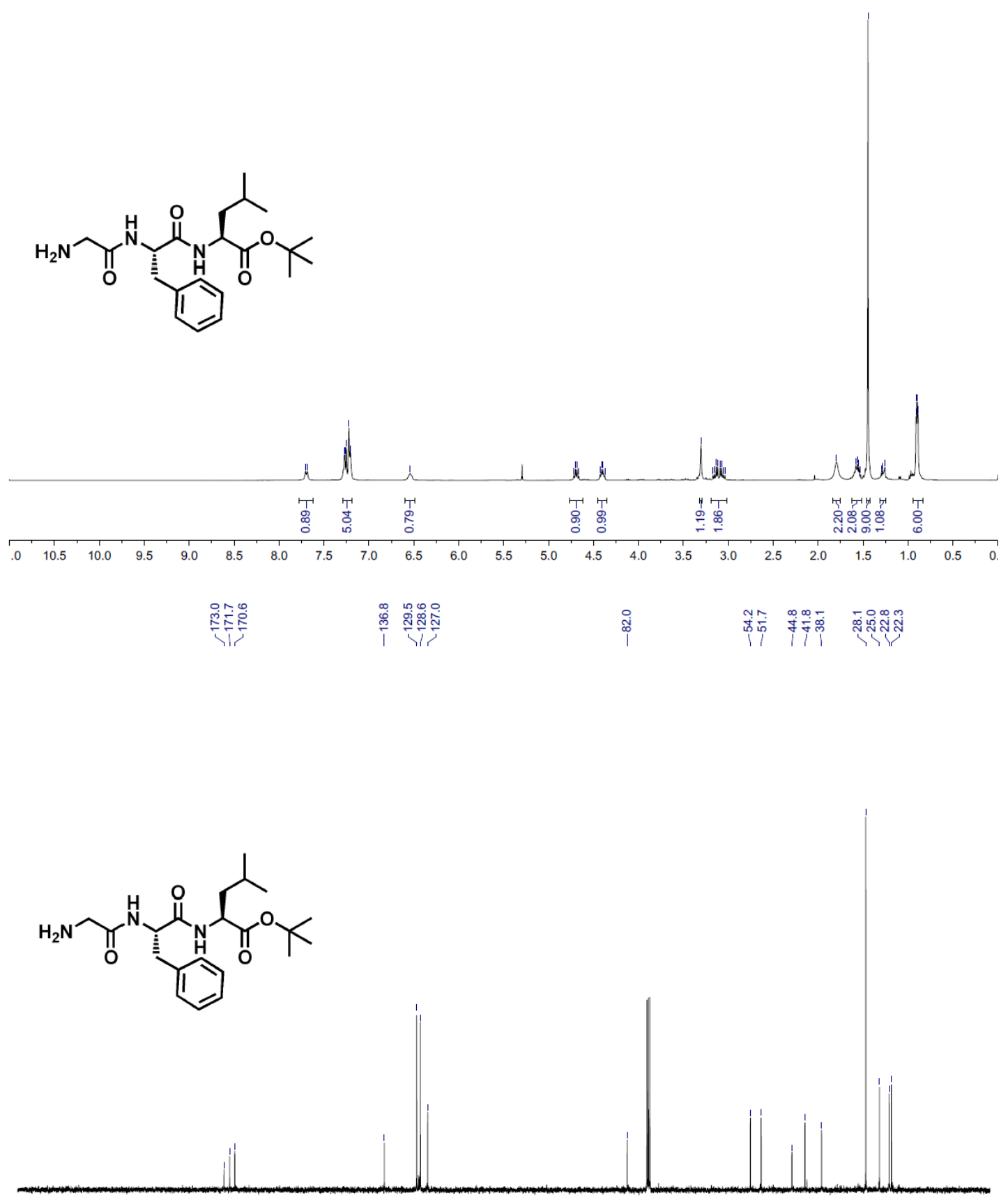

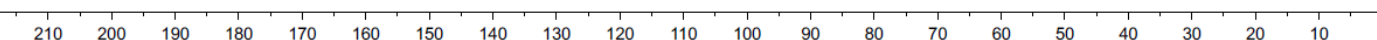



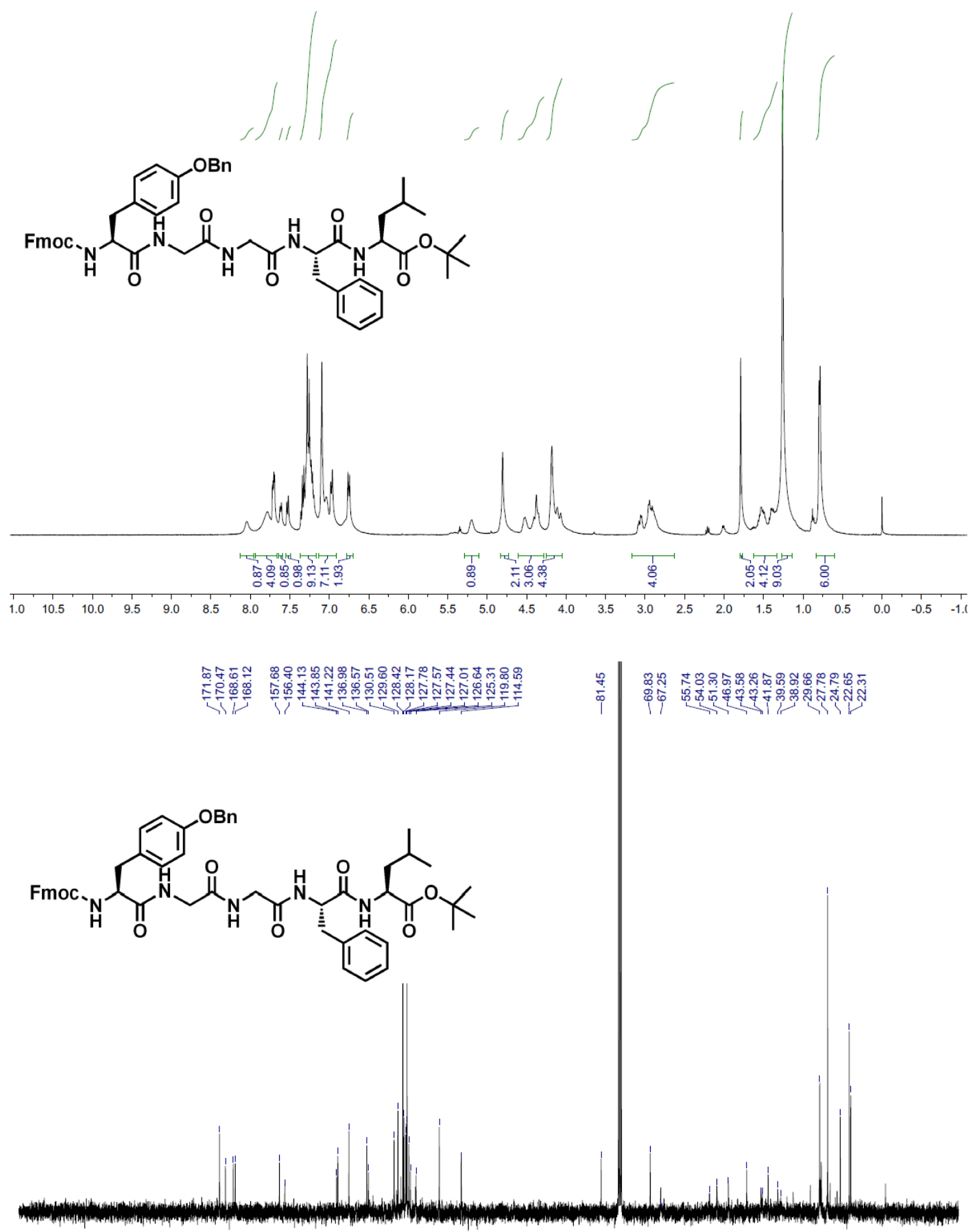

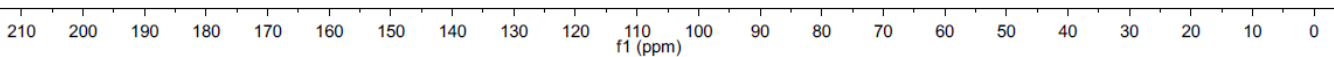

